\title{
A Unified Strategy to Access 2- and 4-Deoxygenated Sugars Enabled by Manganese-promoted 1,2-Radical Migration
}

\author{
Hayden M. Carder, Carolyn E. Suh, and Alison E. Wendlandt* \\ Department of Chemistry, Massachusetts Institute of Technology, Cambridge, MA 02139 \\ Corresponding author.Email: awendlan@mit.edu
}




\section{Table of Contents}

$\begin{array}{ll}\text { 1. General Experimental Methods } & \text { S1 }\end{array}$

2. Materials and Reagents $\quad$ S1

3. Instrumentation $\quad$ S2

4. Synthesis of Catalysts and Substrates $\quad$ S3

A. 4CzIPN $\quad$ S3

B. Rhamnosides $\quad$ S4

$\begin{array}{ll}\text { C. Anhydrosugars } & \text { S8 }\end{array}$

D. Galactosides $\quad \mathrm{S} 10$

E. Mannosides $\quad$ S11

5. General Reaction Procedure of Redox Isomerization $\quad$ S13

A. Reaction procedure for condition optimization $(0.1 \mathrm{mmol}) \quad \mathrm{S} 13$

$\begin{array}{ll}\text { B. Reaction procedure for substrate scope }(0.3 \mathrm{mmol}) & \mathrm{S} 13\end{array}$

C. Reaction procedure for gram-sale $(6.0 \mathrm{mmol}) \quad \mathrm{S} 13$

D. Notes on reaction setup $\quad \mathrm{S} 14$

E. Product characterization $\quad$ S15

6. Derivatizations of Products of Redox Isomerization $\quad$ S21

A. Cis-selective reduction of $2 \quad \mathrm{~S} 21$

$\begin{array}{ll}\text { B. Trans-selective reduction of } 2 & \text { S22 }\end{array}$

$\begin{array}{ll}\text { C/D. Reductive amination of } 2 & \text { S23 }\end{array}$

$\begin{array}{ll}\text { E. Methylation of } 2 & \text { S24 }\end{array}$

$\begin{array}{ll}\text { F/G. Reductive amination of anhydrosugars } & \text { S25 }\end{array}$

7. Condition Optimization for Redox Isomerization of 1 and $6 \quad$ S29

$\begin{array}{ll}\text { A. Variation of standard conditions } & \text { S29 }\end{array}$

B. Screening Lewis bases and Brønsted acids instead of [Mn] $\quad$ S30

$\begin{array}{ll}\text { C. Screening Lewis acids instead of }[\mathrm{Mn}] & \text { S31 }\end{array}$

D. Effect of water $\quad$ S33

8. Mechanistic Studies $\quad$ S34

A. Hydrogen atom abstraction site-selectivity $\quad$ S34

$\begin{array}{ll}\text { B. Mechanistic reactions } & \mathrm{S} 35\end{array}$

C. Investigation of mass balance $\quad$ S36

D. Mn(II) and Mn(III) time course $\quad$ S43

$\begin{array}{ll}\text { E. EPR studies } & \text { S44 }\end{array}$

$\begin{array}{ll}\text { F. UV-Vis studies } & \text { S48 }\end{array}$

$\begin{array}{ll}\text { G. Fluorescence quenching studies } & \text { S49 }\end{array}$

H. Binding studies $\quad$ S51

$\begin{array}{ll}\text { I. Cyclic voltammetry studies } & \text { S53 }\end{array}$

J. Trapping studies $\quad$ S55

9. References $\quad$ S56

$\begin{array}{lr}\text { 10. NMR Spectra } & \text { S58 }\end{array}$ 


\section{General Experimental Methods}

Unless otherwise noted, all catalytic experiments were performed under nitrogen atmosphere by preparing the reactions in nitrogen-filled purge box. Air and/or moisture-sensitive liquids were transferred with stainless steel cannula or glass Hamilton gas-tight syringes fitted with stainlesssteel needles. Reactions were examined by thin-layer chromatography (TLC) on Silica Gel 60 F254 plates (EMD), visualized under UV light $(254 \mathrm{~nm})$ and/or $p$-anisaldehyde and CAM stain, which developed upon heating. Organic solutions were concentrated under reduced pressure on a IKA rotary evaporator. Flash chromatography was performed using SiliaFlash P60 (230-400 mesh, SiliCycle).

\section{Materials and Reagents}

All commercially available reagents were purchased from Sigma-Aldrich, Alfa Aesar, Strem, Oakwood, Matrix Scientific, TCI, Carbosynth, or Chem-Impex and used without purification, unless otherwise indicated. ${ }^{13} \mathrm{C}$ labeled reagents were purchased from Cambridge Isotope Laboratories and used without purification. Extraction and chromatography solvents were reagent grade and used without purification. Reaction solvents, including acetonitrile and dimethyl sulfoxide, were freshly degassed (freeze-pump-thaw) from commercially anhydrous sources, transferred, and stored in a purge box. Deuterated solvents-namely $\mathrm{CDCl}_{3}, \mathrm{CD}_{2} \mathrm{Cl}_{2}, \mathrm{~d}_{6}-\mathrm{DMSO}$, $\mathrm{D}_{2} \mathrm{O}, \mathrm{CD}_{3} \mathrm{OD}$, and $\mathrm{d}_{3}-\mathrm{MeCN}$ (Cambridge Isotope Laboratories) were used without purification. Quinuclidine was purchased from the sources listed above or was prepared according to a previously reported procedure. ${ }^{1}$ Monosaccharide substrates were prepared according to the previously reported procedures (summarized in section 4), and/or purchased through suppliers. C2-deactived silica was used for some substrates to minimize streaking and degradation; it was prepared according to literature precedent. ${ }^{2}$ 


\section{Instrumentation}

Proton nuclear magnetic resonance ( ${ }^{1} \mathrm{H}$ NMR) spectra and proton-decoupled carbon nuclear magnetic resonance $\left({ }^{13} \mathrm{C}\right.$ NMR) spectra were recorded on a Bruker 400 (400 MHz), 500 (500 $\mathrm{MHz})$, and $600(600 \mathrm{MHz})$ spectrometers at $25^{\circ} \mathrm{C}$. All chemical shifts $(\delta)$ are reported in parts per million (ppm) downfield from tetramethylsilane and are referenced to the residual solvent signal of the NMR solvent. Data are represented as follows: chemical shift, multiplicity ( $\mathrm{br}=\mathrm{broad}, \mathrm{s}=$ singlet, $\mathrm{d}=$ doublet, $\mathrm{t}=$ triplet, $\mathrm{q}=$ quartet, quint $=$ quintet, sext $=$ sextet, sept $=$ septet, $\mathrm{m}=$ multiplet), coupling constants $(J)$ in Hertz $(\mathrm{Hz})$, integration. Optical rotations were measured using a $1 \mathrm{dm}$ path length cell with a Jasco Model 1010 digital polarimeter at $589 \mathrm{~nm}$. Infrared (IR) spectra were recorded on an Agilent Cary 630 FTIR Spectrometer. Data are represented as follows: frequency of absorption $\left(\mathrm{cm}^{-1}\right)$, intensity of absorption $(\mathrm{s}=$ strong, $\mathrm{m}=$ medium, $\mathrm{w}=$ weak, $\mathrm{br}=$ broad). High-resolution mass spectrometry was performed on a JEOL Accu TOF Dart at the Department of Chemistry Instrumentation Facility at MIT with the assistance from Dr. Mohan Kumar. Stern-Volmer quenching experiments were performed on Varian Cary Eclipse fluorescence spectrophotometer. UV-Vis spectroscopy was performed on Avantes spectrometer (AvaLight-DHc and AvaSpec 204B). EPR spectra of frozen solutions under nonsaturating conditions were recorded on a Bruker EMX spectrometer at $50 \mathrm{~K}$ with power of $25 \mu \mathrm{W}$. EPR data was visualized and analyzed using EasySpin on MATLAB. Cyclic voltammetry data were measured with a Pine Research (AFP1) potentiostat. 


\section{Synthesis of Catalysts and Substrates}

A) 4 CzIPN

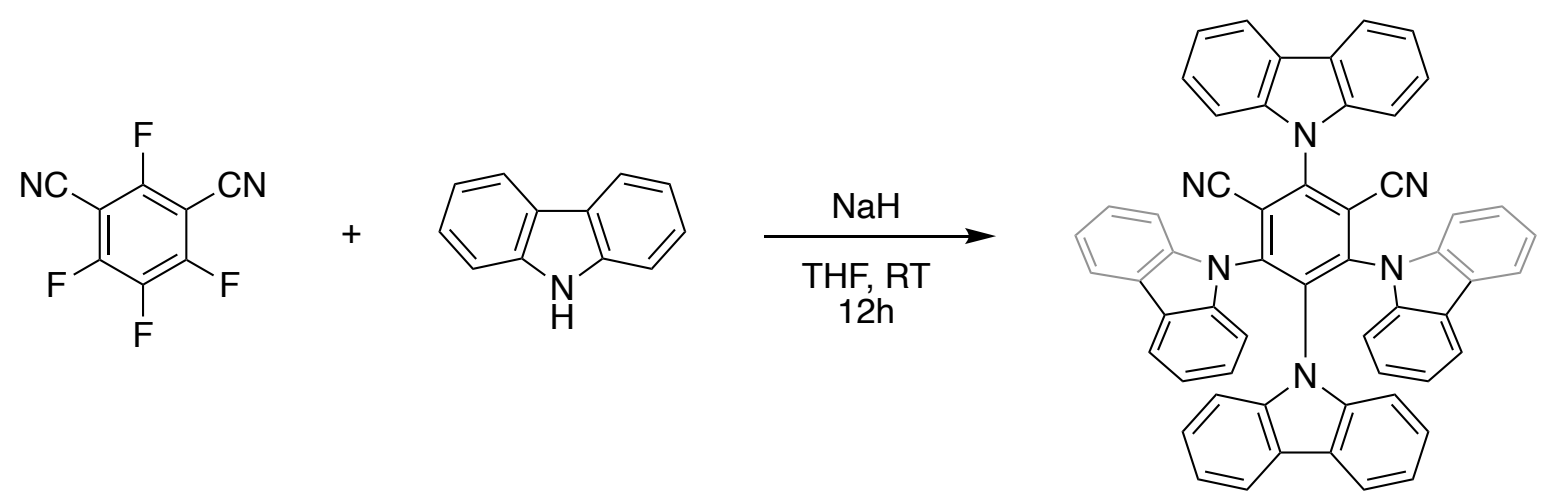

Catalyst 4CzIPN was prepared according to the previously reported procedures. ${ }^{3}$ To a $250 \mathrm{~mL}$ oven-dried round bottom flask, $\mathrm{NaH}(60 \%$ in mineral oil, $1.20 \mathrm{~g}, 30.0 \mathrm{mmol})$ was added slowly to the solution of carbazole $(3.34 \mathrm{~g}, 20.0 \mathrm{mmol})$ in dry THF $(80 \mathrm{~mL})$ under nitrogen atmosphere at room temperature. After $30 \mathrm{~min}$, tetrafluoroisophthalonitrile $(0.80 \mathrm{~g}, 4.00 \mathrm{mmol})$ was added. the resulting reaction mixture was further stirred at room temperature for $12 \mathrm{~h}$ and quenched by slow addition of $4.0 \mathrm{~mL}$ water. The resulting mixture was then concentrated under reduced pressure and washed with water and $\mathrm{EtOH}$ to get rid of the majority of the remaining carbazole. The crude solid will be green/yellow and turn a bright yellow upon complete removal of impurities. If necessary, the crude product was purified by column chromatography on silica gel with $\mathrm{CH}_{2} \mathrm{Cl}_{2}$ /hexane to give $2.91 \mathrm{~g} \mathrm{(92 \% )} \mathrm{as} \mathrm{yellow} \mathrm{solid.}{ }^{1} \mathrm{H}$ NMR $\left(400 \mathrm{MHz}, \mathrm{CD}_{2} \mathrm{Cl}_{2}\right) \delta 8.33(\mathrm{~d}, J=7.7 \mathrm{~Hz}, 2 \mathrm{H}), 7.84$ -7.77 (m, 8H), $7.60-7.56(\mathrm{~m}, 2 \mathrm{H}), 7.44$ (d, J=7.6 Hz, 2H), $7.35-7.31$ (m, 4H), $7.21-7.16(\mathrm{~m}$, $8 \mathrm{H}), 6.99$ (d, $J=8.2 \mathrm{~Hz}, 2 \mathrm{H}), 6.91$ (t, $J=7.4 \mathrm{~Hz}, 2 \mathrm{H}), 6.74(\mathrm{t}, J=7.7 \mathrm{~Hz}, 2 \mathrm{H}) .{ }^{13} \mathrm{C}$ NMR $(100$ $\left.\mathrm{MHz}, \mathrm{CD}_{2} \mathrm{Cl}_{2}\right) \delta 145.68,145.07,140.30,138.62,137.51,135.49,127.43,126.28,125.34,125.17$, $124.76,124.16,122.85,122.35,121.74,121.46,120.81,120.02,116.99,112.17,110.46,110.04$. 


\section{B) Rhamnosides}
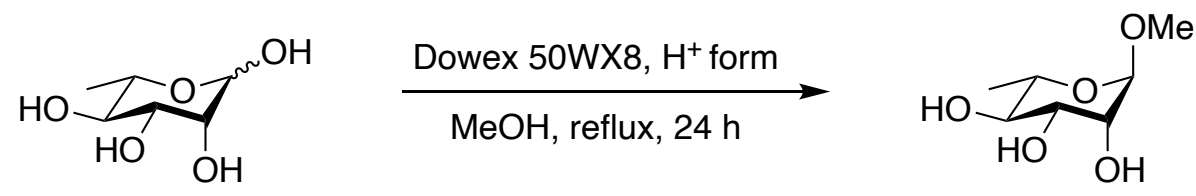

1

L- $\alpha$-methylrhamnoside 1 was prepared according to the previously reported procedures. ${ }^{4}$ Rhamnose monohydrate (30.0 g, $182.7 \mathrm{mmol})$, Dowex ${ }^{\mathrm{TM}} 50 \mathrm{WX} 8-100$ ion-exchange resin $(20 \mathrm{~g})$, and $250 \mathrm{~mL}$ of $\mathrm{MeOH}$ (reagent grade) was added to a $500 \mathrm{~mL}$ round bottom flask equipped with a stir bar. The reaction was stirred and refluxed at $70{ }^{\circ} \mathrm{C}$ for $24 \mathrm{~h}$. The reaction was cooled to room temperature. The resin was filtered and washed with $\mathrm{MeOH}$. The resulting filtrate was concentrated under reduced pressure. The resulting syrup dissolved in hot ethyl acetate was filtered through a silica plug. The material was concentrated under reduced pressure and the resulting syrup was purified by recrystallization from ethyl acetate $(20.95 \mathrm{~g}, 0.118 \mathrm{mmol}, 64 \%)$. The NMR spectra are consistent with those reported in the literature. ${ }^{5}{ }^{1} \mathrm{H}$ NMR $(400 \mathrm{MHz}, \mathrm{MeOD}) \delta 4.55(\mathrm{~d}, J=1.7$ $\mathrm{Hz}, 1 \mathrm{H}), 3.77$ (dd, $J=3.4,1.6 \mathrm{~Hz}, 1 \mathrm{H}), 3.60$ (dd, $J=9.4,3.5 \mathrm{~Hz}, 1 \mathrm{H}), 3.56-3.50$ (m, 1H), 3.34 (s, 3H), 1.27 (d, $J=6.2 \mathrm{~Hz}, 3 \mathrm{H}) .{ }^{13} \mathrm{C}$ NMR (400 MHz, MeOD) $\delta 101.4,72.55,71.0,70.8,68.2$, $53.7,16.6$. 


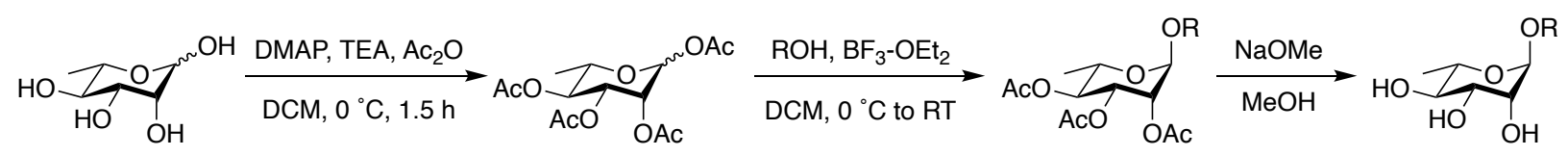

L- $\alpha$-cyclohexylrhamnoside S1, L- $\alpha$-benzylrhamnoside S2, and L- $\alpha$-(4-F)benzylrhamnoside

S3: peracetylated rhamnose was prepared according to the previously reported procedures. ${ }^{6}$ Rhamnose (10.0 g, $54.9 \mathrm{mmol})$, DMAP (1.34 g, $11.0 \mathrm{mmol})$, and a PTFE magnetic stir bar were added to a $250 \mathrm{~mL}$ round bottom flask. After purging the flask with nitrogen, $50 \mathrm{~mL}$ dry DCM was added. The flask was then cooled to $0{ }^{\circ} \mathrm{C}$. TEA $(61 \mathrm{~mL}, 439 \mathrm{mmol})$ was added over $5 \mathrm{~min}$ followed by $\mathrm{Ac}_{2} \mathrm{O}(36 \mathrm{~mL}, 384 \mathrm{mmol})$ over $15 \mathrm{~min}$. The resulting mixture was stirred for $1.5 \mathrm{~h}$ at $0{ }^{\circ} \mathrm{C}$ and then quenched by $5 \mathrm{~mL}$ of $\mathrm{MeOH}$. The mixture was then concentrated under reduced pressure. The solution was transferred to a separatory funnel, diluted with $100 \mathrm{~mL}$ EtOAc, and extracted with brine $(3 \times 20 \mathrm{~mL})$. The combined aqueous extracts were back-extracted with EtOAc $(3 \times 50 \mathrm{~mL})$. The organic layers were combined, washed with $5 \% \mathrm{HCl}(25 \mathrm{~mL})$ then sat. $\mathrm{NaHCO}_{3}$ until the organic layer was $\mathrm{pH}>7$. The organic layer was extracted once more with brine $(25 \mathrm{~mL})$, and subsequently dried over $\mathrm{MgSO}_{4}$, filtered, and concentrated in vacuo. The resulting yellow, viscous syrup was carried forward without purification $(94 \%, 17.1 \mathrm{~g}, 8: 1 \alpha / \beta) .{ }^{1} \mathrm{H} \mathrm{NMR}(400 \mathrm{MHz}$, $\left.\mathrm{CDCl}_{3}\right) \delta 6.01(\mathrm{~d}, J=1.9 \mathrm{~Hz}, 1 \mathrm{H}), 5.30(\mathrm{dd}, J=10.1,3.5 \mathrm{~Hz}, 1 \mathrm{H}), 5.27-5.22(\mathrm{~m}, 1 \mathrm{H}), 5.12(\mathrm{t}, J$ $=10.0 \mathrm{~Hz}, 1 \mathrm{H}), 3.94(\mathrm{dq}, J=9.7,6.2 \mathrm{~Hz}, 1 \mathrm{H}), 2.17(\mathrm{~s}, 3 \mathrm{H}), 2.16(\mathrm{~s}, 3 \mathrm{H}), 2.06(\mathrm{~s}, 3 \mathrm{H}), 2.04(\mathrm{~s}$, $3 \mathrm{H}), 1.25(\mathrm{~d}, J=5.2 \mathrm{~Hz}, 3 \mathrm{H})$.

A general glycosylation strategy was used to synthesize L- $\alpha$-rhamnosides with variation of the aglycone. In a vial equipped with a stir bar and septum cap was added the material from the previous step (peracetylated rhamnose $2 \mathrm{~g}, 6.0 \mathrm{mmol}$ ); after purging the vial with nitrogen, $10 \mathrm{~mL}$ DCM and the appropriate acceptor ( 1 equiv, $6.0 \mathrm{mmol}$ ) was added. The vial was cooled to $0{ }^{\circ} \mathrm{C}$. $\mathrm{BF}_{3}-\mathrm{OEt}_{2}$ (5 equiv, $3.7 \mathrm{~mL}, 30.1 \mathrm{mmol}$ ) was added drop wise. The resulting mixture was stirred for $2 \mathrm{~h}$ at $0{ }^{\circ} \mathrm{C}$ and then stirred overnight at RT. After this time, the mixture was diluted with DCM and water. The organic layer was washed with $1 \mathrm{M} \mathrm{HCl}$, sat. $\mathrm{NaHCO}_{3}$, and brine. The washed organic layer was then dried over $\mathrm{Na}_{2} \mathrm{SO}_{4}$, filtered, and concentrated in vacuo. The crude material was then purified by column chromatography to afford the desired product (Hex/EtOAc 2:1). 


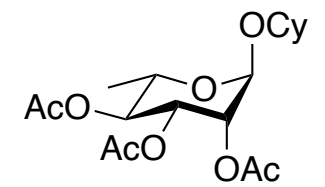

S1, L-a-cyclohexylrhamnose triacetate ${ }^{7}: 1.59 \mathrm{~g}, 4.27 \mathrm{mmol}, 71 \%$; ${ }^{1} \mathrm{H}$ NMR $\left(500 \mathrm{MHz} \mathrm{CDCl}_{3}\right) \delta 5.33(\mathrm{dd}, J=10.1,3.5 \mathrm{~Hz}, 1 \mathrm{H}), 5.18(\mathrm{dd}, J=3.5,1.8 \mathrm{~Hz}, 1 \mathrm{H}), 5.05(\mathrm{t}, J=$ $9.9 \mathrm{~Hz}, 1 \mathrm{H}), 4.86(\mathrm{~d}, J=1.8 \mathrm{~Hz}, 1 \mathrm{H}), 4.02-3.91(\mathrm{~m}, 1 \mathrm{H}), 3.64-3.50(\mathrm{~m}, 1 \mathrm{H}), 2.15(\mathrm{~s}, 3 \mathrm{H}), 2.04$ $(\mathrm{d}, J=1.1 \mathrm{~Hz}, 3 \mathrm{H}), 1.98(\mathrm{~s}, 3 \mathrm{H}), 1.93-1.79(\mathrm{~m}, 2 \mathrm{H}), 1.79-1.66(\mathrm{~m}, 2 \mathrm{H}), 1.62-1.22(\mathrm{~m}, 6 \mathrm{H})$, $1.20(\mathrm{~d}, J=6.3 \mathrm{~Hz}, 3 \mathrm{H})$.

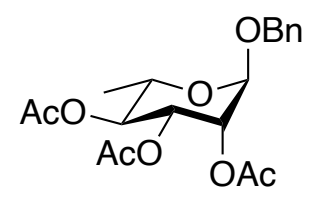

S2, L- $\alpha$-benzylrhamnose triacetate ${ }^{8}: 1.65 \mathrm{~g}, 4.34 \mathrm{mmol}, 72 \%$; ${ }^{1} \mathrm{H}$ NMR (500 $\left.\mathrm{MHz}, \mathrm{CDCl}_{3}\right) \delta 7.40-7.28(\mathrm{~m}, 5 \mathrm{H}), 5.34(\mathrm{dd}, J=10.0,3.5 \mathrm{~Hz}, 1 \mathrm{H}), 5.29(\mathrm{dd}, J=3.5,1.8 \mathrm{~Hz}$, $1 \mathrm{H}), 5.08(\mathrm{t}, J=9.9 \mathrm{~Hz}, 1 \mathrm{H}), 4.81(\mathrm{~d}, J=1.8 \mathrm{~Hz}, 1 \mathrm{H}), 4.71(\mathrm{~d}, J=12.0 \mathrm{~Hz}, 1 \mathrm{H}), 4.55(\mathrm{~d}, J=12.0$ $\mathrm{Hz}, 1 \mathrm{H}), 3.90(\mathrm{dq}, J=9.8,6.3 \mathrm{~Hz}, 1 \mathrm{H}), 2.13(\mathrm{~s}, 3 \mathrm{H}), 2.04(\mathrm{~s}, 3 \mathrm{H}), 1.98(\mathrm{~s}, 3 \mathrm{H}), 1.21(\mathrm{~d}, J=6.3$ $\mathrm{Hz}, 3 \mathrm{H})$.

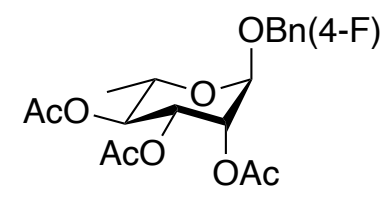

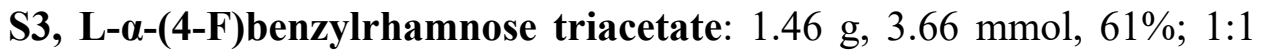
EtOAc/Hex, $\mathrm{R}_{\mathrm{f}}=0.17 .[\alpha]^{20} \mathrm{D}=-90.8(\mathrm{c}=1, \mathrm{MeCN}) .{ }^{1} \mathrm{H} \mathrm{NMR}\left(400 \mathrm{MHz}, \mathrm{CDCl}_{3}\right) \delta 7.34-7.28$ $(\mathrm{m}, 2 \mathrm{H}), 7.04(\mathrm{t}, J=8.7 \mathrm{~Hz}, 2 \mathrm{H}), 5.35-5.28(\mathrm{~m}, 1 \mathrm{H}), 5.26(\mathrm{dd}, J=3.5,1.8 \mathrm{~Hz}, 1 \mathrm{H}), 5.07(\mathrm{t}, J=$ $9.9 \mathrm{~Hz}, 1 \mathrm{H}), 4.78(\mathrm{~d}, J=1.7 \mathrm{~Hz}, 1 \mathrm{H}), 4.66(\mathrm{~d}, J=11.8 \mathrm{~Hz}, 1 \mathrm{H}), 4.51(\mathrm{~d}, J=11.9 \mathrm{~Hz}, 1 \mathrm{H}), 3.87$ $(\mathrm{dq}, J=9.7,6.3 \mathrm{~Hz}, 1 \mathrm{H}), 2.13(\mathrm{~s}, 3 \mathrm{H}), 2.04(\mathrm{~s}, 3 \mathrm{H}), 1.98(\mathrm{~s}, 3 \mathrm{H}), 1.20(\mathrm{~d}, J=6.3 \mathrm{~Hz}, 3 \mathrm{H}) .{ }^{13} \mathrm{C}$ NMR $\left(100 \mathrm{MHz}, \mathrm{CDCl}_{3}\right) \delta 170.25,170.13,163.91,161.46,132.53,132.50,130.04,129.96$, $115.67,115.45,96.72,71.20,69.98,69.26,68.93,66.74,21.04,20.93,20.85,17.52 .{ }^{19} \mathrm{~F}$ NMR $\left(376 \mathrm{MHz}, \mathrm{CDCl}_{3}\right) \delta-114.06$ (ddd, $J=14.1,8.7,5.4 \mathrm{~Hz}$ ). IR (neat, $\mathrm{cm}^{-1}$ ): 3445.2 (br), 2937.1 (br), 1736.9 (s), 1602.8 (w), 1513.3 (s), 1222.6 (s), 1121.9 (s), 1058.6 (s), 998.9 (s), 827.5 (w). HRMS $(\mathrm{DART})\left(\left[\mathrm{M}+\mathrm{NH}_{4}\right]^{+}\right)$Calcd. For C19H27NO8F: 416.17152. Found 416.17169. 
The sugars were deacetylated under basic conditions. In a vial equipped with a stir bar and septum cap was added the material from the previous step ( $\alpha$-rhamnosides triacetates, $3 \mathrm{mmol}$ ) and $\mathrm{NaOMe}(0.162 \mathrm{~g}, 3 \mathrm{mmol})$; after purging the vial with nitrogen, $15 \mathrm{~mL}$ anhydrous $\mathrm{MeOH}$ was added. The resulting mixture was stirred at RT and monitored by TLC $(2 \mathrm{~h})$. Upon completion, the mixture was concentrated in vacuo and purified by column chromatography to afford the desired product (DCM/MeOH 8:1).

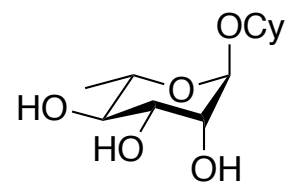

S4, L-a-cyclohexylrhamnose ${ }^{7}: 0.690 \mathrm{~g}, 2.80 \mathrm{mmol}, 93 \%$; ${ }^{1} \mathrm{H}$ NMR $(400 \mathrm{MHz}$, $\left.\mathrm{CDCl}_{3}\right) \delta 4.93(\mathrm{~s}, 1 \mathrm{H}), 3.89(\mathrm{~d}, J=3.4 \mathrm{~Hz}, 1 \mathrm{H}), 3.85-3.70(\mathrm{~m}, 2 \mathrm{H}), 3.65-3.54(\mathrm{~m}, 1 \mathrm{H}), 3.44(\mathrm{t}$, $J=9.4 \mathrm{~Hz}, 1 \mathrm{H}), 1.85(\mathrm{br}, 2 \mathrm{H}), 1.73(\mathrm{br}, 2 \mathrm{H}), 1.53(\mathrm{br}, 1 \mathrm{H}), 1.45-1.15(\mathrm{~m}, 8 \mathrm{H})$.

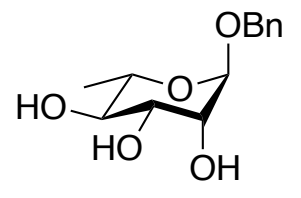

S5, L-a-benzylrhamnose ${ }^{8}: 0.702 \mathrm{~g}, 2.76 \mathrm{mmol}, 92 \%$; ${ }^{1} \mathrm{H}$ NMR $(400 \mathrm{MHz}$, $\left.\mathrm{CDCl}_{3}\right) \delta 7.40-7.27(\mathrm{~m}, 5 \mathrm{H}), 4.86(\mathrm{~s}, 1 \mathrm{H}), 4.71(\mathrm{~d}, J=11.9 \mathrm{~Hz}, 1 \mathrm{H}), 4.50(\mathrm{~d}, J=11.9 \mathrm{~Hz}, 1 \mathrm{H})$, $4.03-3.90(\mathrm{~m}, 1 \mathrm{H}), 3.81(\mathrm{dd}, J=9.4,3.4 \mathrm{~Hz}, 1 \mathrm{H}), 3.77-3.65(\mathrm{~m}, 1 \mathrm{H}), 3.45(\mathrm{~d}, J=9.4 \mathrm{~Hz}, 1 \mathrm{H})$, $1.32(\mathrm{~d}, J=6.2 \mathrm{~Hz}, 3 \mathrm{H})$.

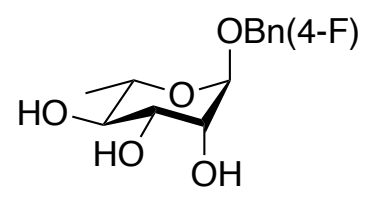

S6, L- $\boldsymbol{\alpha}$-(4-F)benzylrhamnose: $0.630 \mathrm{~g}, 2.31 \mathrm{mmol}, 77 \%$; 10:1 $\mathrm{DCM} / \mathrm{MeOH}, \mathrm{R}_{\mathrm{f}}=0.26 .[\alpha]^{20}{ }_{\mathrm{D}}=-83.2(\mathrm{c}=1, \mathrm{MeCN}) .{ }^{1} \mathrm{H}$ NMR $(400 \mathrm{MHz}, \mathrm{MeOD}) \delta 7.63-7.28$ (m, 2H), $7.24-6.86(\mathrm{~m}, 2 \mathrm{H}), 4.75(\mathrm{~d}, J=1.7 \mathrm{~Hz}, 1 \mathrm{H}), 4.66(\mathrm{~d}, J=11.8 \mathrm{~Hz}, 1 \mathrm{H}), 4.49$ (d, $J=11.8$ $\mathrm{Hz}, 1 \mathrm{H}), 3.81(\mathrm{dd}, J=3.5,1.7 \mathrm{~Hz}, 1 \mathrm{H}), 3.66(\mathrm{dd}, J=9.5,3.4 \mathrm{~Hz}, 1 \mathrm{H}), 3.60(\mathrm{dq}, J=9.5,6.3 \mathrm{~Hz}$, $1 \mathrm{H}), 3.38(\mathrm{t}, J=9.5 \mathrm{~Hz}, 1 \mathrm{H}), 1.26(\mathrm{~d}, J=6.2 \mathrm{~Hz}, 3 \mathrm{H}) .{ }^{13} \mathrm{C} \mathrm{NMR}(100 \mathrm{MHz}, \mathrm{MeOD}) \delta 165.05$, 162.62, 135.21, 135.18, 131.09, 131.00, 116.17, 115.96, 100.88, 73.97, 72.41, 72.28, 70.05, 69.29, 17.99. ${ }^{19} \mathrm{~F}$ NMR (376 MHz, MeOD) $\delta-117.77$ (ddd, $J=14.3,9.0,5.5 \mathrm{~Hz}$ ). IR (neat, $\mathrm{cm}^{-1}$ ): 3395.6 (br, s), 2929.7 (br, w), 1602.8 (w), 1513.3 (s), 1222.6 (s), 1129.4 (w), 1051.1 (s). HRMS (DART) $\left(\left[\mathrm{M}+\mathrm{NH}_{4}\right]^{+}\right)$Calcd. For C13H21NO5F: 290.13983. Found 290.14089. 


\section{C) Anhydrosugars}

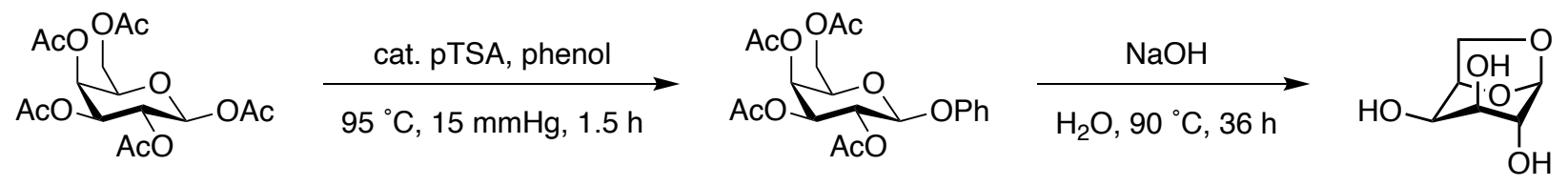

D-anhydrogalactose 9 was prepared according to the previously reported procedures. ${ }^{9} \beta$-Dgalactose pentaacetate $(19.5 \mathrm{~g}, 50.0 \mathrm{mmol}), p$-toluenesulfonic acid (0.285 g, $1.5 \mathrm{mmol})$, phenol (16.47, $175.0 \mathrm{mmol})$, and a PTFE magnetic stir bar were added to a $250 \mathrm{~mL}$ round bottom flask. The flask was heated to $95^{\circ} \mathrm{C}$ in an oil bath while under vacuum $(15 \mathrm{mmHg}$, pressure was regulated using a Buchi Vacuum Controller V 805). Upon heating the solids melted together. After 1.5 hours the reaction was diluted with DCM and washed with $0.5 \mathrm{M} \mathrm{NaOH}(300 \mathrm{ml})$ and water $(300 \mathrm{ml})$. The organic layer was dried over $\mathrm{Na}_{2} \mathrm{SO}_{4}$, filtered, and concentrated in vacuo. The resulting oil or solid was recrystallized from reagent ethanol to achieve white crystals $(1: 10 \alpha / \beta), 17.7 \mathrm{~g}, 41.7$ mmol, 83\%. The NMR spectra are consistent with those reported in the literature. ${ }^{10}{ }^{1} \mathrm{H}$ NMR (400 $\left.\mathrm{MHz}, \mathrm{CDCl}_{3}\right) \delta 7.34-7.27(\mathrm{~m}, 2 \mathrm{H}), 7.11-7.04(\mathrm{~m}, 1 \mathrm{H}), 7.03-6.98(\mathrm{~m}, 2 \mathrm{H}), 5.49$ (dd, $J=10.5$, $8.0 \mathrm{~Hz}, 1 \mathrm{H}), 5.46(\mathrm{dd}, J=3.5,1.1 \mathrm{~Hz}, 1 \mathrm{H}), 5.11(\mathrm{dd}, J=10.4,3.4 \mathrm{~Hz}, 1 \mathrm{H}), 5.05$ (d, $J=7.9 \mathrm{~Hz}$, 1H), $4.24(\mathrm{dd}, J=11.3,7.0 \mathrm{~Hz}, 1 \mathrm{H}), 4.16(\mathrm{dd}, J=11.3,6.3 \mathrm{~Hz}, 1 \mathrm{H}), 4.06(\mathrm{td}, J=6.7,1.2 \mathrm{~Hz}, 1 \mathrm{H})$, $2.18(\mathrm{~s}, 3 \mathrm{H}), 2.07(\mathrm{~s}, 3 \mathrm{H}), 2.06(\mathrm{~s}, 3 \mathrm{H}), 2.02(\mathrm{~s}, 3 \mathrm{H})$.

$\mathrm{NaOH}$ pellets (15 g, $375 \mathrm{mmol})$, water $(300 \mathrm{~mL})$, and a PTFE magnetic stir bar were added to a $500 \mathrm{~mL}$ round bottom flask. The solution was stirred until homogenous. 1-phenyl-tetraacetyl$\beta$-D-galactopyranoside $(10.6 \mathrm{~g}, 25 \mathrm{mmol})$ was added and the mixture was heated to $90{ }^{\circ} \mathrm{C}$ for 36 h. The reaction mixture was cooled to $0{ }^{\circ} \mathrm{C}$ and adjusted to $\mathrm{pH}=3$ with aqueous $\mathrm{HCl}$. The mixture

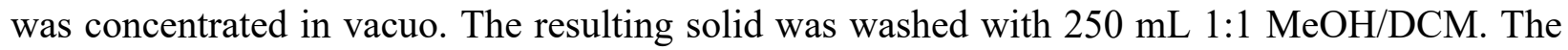
filtrate was collected and concentrated in vacuo. The crude product was subjected to column chromatography to remove dissolved salts and other impurities (DCM/MeOH). Remaining impurities, such as 1-phenyl- $\alpha$-D-galactopyranoside, can be removed by recrystallization with $\mathrm{MeOH} / \mathrm{MeCN}$ (solid dissolved in minimal amount of hot $\mathrm{MeOH}$ and subsequently dilute 1:1 with $\mathrm{MeCN}) 1.05 \mathrm{~g}, 6.50 \mathrm{mmol}, 27 \% .{ }^{1} \mathrm{H}$ NMR (400 MHz, MeOD) $\delta 5.24$ (t, $\left.J=1.6 \mathrm{~Hz}, 1 \mathrm{H}\right), 4.33$ (d, $J=7.1 \mathrm{~Hz}, 1 \mathrm{H}), 4.29(\mathrm{td}, J=4.7,4.3,1.3 \mathrm{~Hz}, 1 \mathrm{H}), 3.99-3.92(\mathrm{~m}, 1 \mathrm{H}), 3.84(\mathrm{dq}, J=4.9,1.5 \mathrm{~Hz}$, $1 \mathrm{H}), 3.65(\mathrm{t}, J=1.8 \mathrm{~Hz}, 1 \mathrm{H}), 3.53(\mathrm{ddd}, J=6.3,5.0,1.4 \mathrm{~Hz}, 1 \mathrm{H})$. 


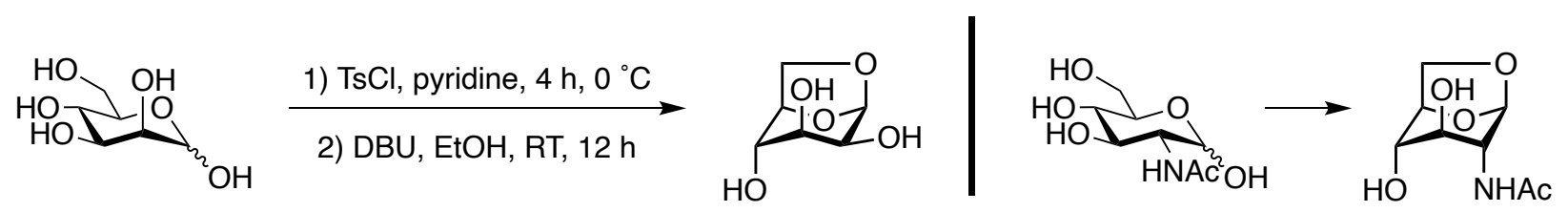

D-anhydromannose 11 and D-anhydroglucoseamine 17 were prepared according to an adapted procedure. ${ }^{11}$ Free sugar (mannose or N-acetyl glucosamine) (54 mmol, 1 equiv) and a PTFE magnetic stir bar were added to a $500 \mathrm{~mL}$ round bottom flask. After purging the flask with nitrogen, $120 \mathrm{~mL}$ dry pyridine was added. The flask was then cooled to $0{ }^{\circ} \mathrm{C}$. TsCl $(12.4 \mathrm{~g}, 65 \mathrm{mmol}, 1.2$ equiv) in $60 \mathrm{~mL}$ pyridine was added over $1 \mathrm{~h}$. The resulting mixture was stirred for $4 \mathrm{~h}$ at $0{ }^{\circ} \mathrm{C}$ and then quenched by $10 \mathrm{~mL}$ of $\mathrm{MeOH}$. The mixture was concentrated under reduced pressure. 400 $\mathrm{mL}$ reagent ethanol and DBU $(21 \mathrm{~mL}, 140 \mathrm{mmol}, 2.6$ equiv) were added to the resulting oil. The solution was stirred overnight. The mixture was then concentrated under reduced pressure $\left(50{ }^{\circ} \mathrm{C}\right)$. The resulting oil was subjected to column chromatography (0-15\% MeOH in EtOAc). Remaining impurities can be removed by recrystallization from a minimal amount of hot $\mathrm{MeOH}$.

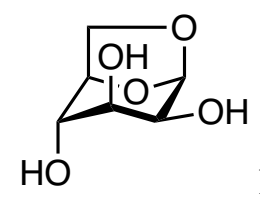

11, D-anhydromannose: $2.14 \mathrm{~g}, 10.5 \mathrm{mmol}, 19 \% .{ }^{1} \mathrm{H}$ NMR (400 MHz, MeOD) $\delta$ $5.23(\mathrm{t}, J=1.7 \mathrm{~Hz}, 1 \mathrm{H}), 4.46-4.39(\mathrm{~m}, 1 \mathrm{H}), 4.20(\mathrm{dd}, J=7.1,1.1 \mathrm{~Hz}, 1 \mathrm{H}), 3.83(\mathrm{dq}, J=5.2,1.7$ $\mathrm{Hz}, 1 \mathrm{H}), 3.77$ (d, $J=1.9 \mathrm{~Hz}, 1 \mathrm{H}), 3.68-3.61(\mathrm{~m}, 2 \mathrm{H})$.

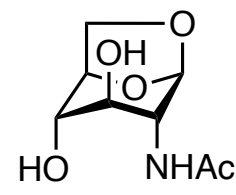

17, D-anhydroglucoseamine ${ }^{11}: 2.50 \mathrm{~g}, 15.4 \mathrm{mmol}, 28 \%{ }^{1} \mathrm{H}$ NMR (400 MHz, MeOD) $\delta 5.26(\mathrm{~d}, J=1.7 \mathrm{~Hz}, 1 \mathrm{H}), 4.58-4.44(\mathrm{~m}, 1 \mathrm{H}), 4.18(\mathrm{dd}, J=7.2,1.0 \mathrm{~Hz}, 1 \mathrm{H}), 3.83(\mathrm{~d}, J$ $=1.8 \mathrm{~Hz}, 1 \mathrm{H}), 3.68(\mathrm{dd}, J=7.2,5.7 \mathrm{~Hz}, 1 \mathrm{H}), 3.61-3.50(\mathrm{~m}, 2 \mathrm{H}), 1.98(\mathrm{~s}, 3 \mathrm{H})$. 


\section{D) Galactosides}

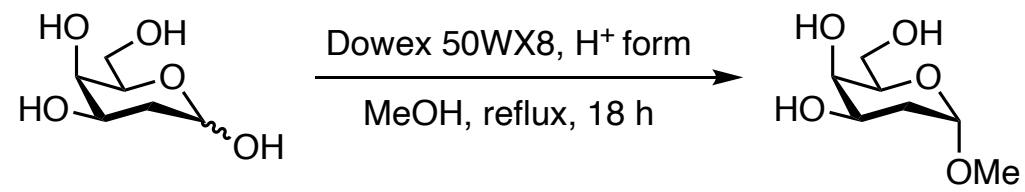

D-2-deoxy- $\boldsymbol{\alpha}$-methylgalactoside $\mathbf{2 0}$ was prepared according to the previously reported procedures. 2-deoxy galactose ( $3 \mathrm{~g}, 18.3 \mathrm{mmol})$ and Dowex ${ }^{\mathrm{TM}} 50 \mathrm{WX} 8-100$ ion-exchange resin (3 g) were added to a $40 \mathrm{~mL}$ vial equipped with a stir bar. The vial was capped with a septum cap and purged with nitrogen for $10 \mathrm{~min} .15 \mathrm{~mL}$ anhydrous methanol was added via syringe. The reaction was heated at $65{ }^{\circ} \mathrm{C}$ and stirred for $18 \mathrm{~h}$. The reaction was cooled to room temperature. The resin was filtered and washed with $\mathrm{MeOH}$. The resulting filtrate was concentrated under reduced pressure. The resulting oil was subjected to column chromatography $(0-20 \% \mathrm{MeOH}$ in DCM), 4:1 $\alpha / \beta$. Pure 2-deoxy- $\alpha$-methylgalactoside was obtained by recrystallization from hot ethyl acetate ( 20 mg/mL), $1.40 \mathrm{~g}, 7.80 \mathrm{mmol}, 43 \%$. ${ }^{1} \mathrm{H}$ NMR (400 MHz, MeOD) $\delta 4.80$ (d, $J=3.7 \mathrm{~Hz}$, $1 \mathrm{H}), 3.90(\mathrm{ddd}, J=12.0,5.1,3.1 \mathrm{~Hz}, 1 \mathrm{H}), 3.76(\mathrm{~d}, J=3.0 \mathrm{~Hz}, 1 \mathrm{H}), 3.74-3.65(\mathrm{~m}, 3 \mathrm{H}), 3.33$ (s, 3H), 1.93 (ddd, $J=12.4,3.8 \mathrm{~Hz}, 1 \mathrm{H}), 1.75$ (dd, $J=12.8,5.1 \mathrm{~Hz}, 1 \mathrm{H})$.

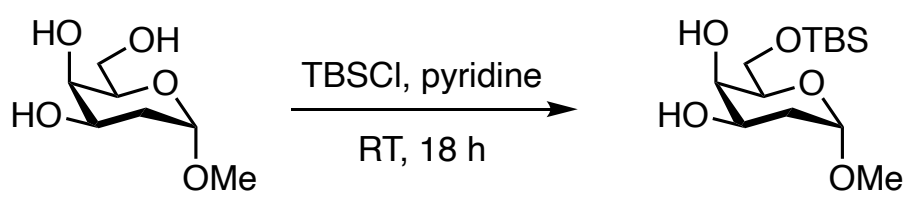

D-O6-TBS-2-deoxy- $\boldsymbol{\alpha}$-methylgalactoside $\mathbf{S} 7$ was prepared according to according to an adapted procedure. $^{12,13}$ 2-deoxy- $\alpha$-methylgalactoside $(0.356 \mathrm{~g}, 2 \mathrm{mmol})$ and $\mathrm{TBSCl}(0.301 \mathrm{~g}, 2 \mathrm{mmol})$ were added to a 2 dram vial equipped with a stir bar. The vial was capped with a septum cap and purged with nitrogen for $10 \mathrm{~min} .3 \mathrm{~mL}$ anhydrous pyridine was added via syringe. The reaction was stirred for $18 \mathrm{~h}$ at RT and subsequently concentrated under reduced pressure. The resulting oil was diluted with EtOAc, transferred to a separatory funnel, and extracted with water and brine. The organic layer was subsequently dried over $\mathrm{MgSO}_{4}$, filtered, and concentrated in vacuo. The product was obtained as a white solid, $0.169 \mathrm{~g}, 0.58 \mathrm{mmol}, 30 \% .{ }^{1} \mathrm{H} \mathrm{NMR}\left(400 \mathrm{MHz}, \mathrm{CDCl}_{3}\right) \delta 4.82(\mathrm{dd}, J=$ 3.4, $1.8 \mathrm{~Hz}, 1 \mathrm{H}), 4.02-3.92(\mathrm{~m}, 2 \mathrm{H}), 3.92-3.84(\mathrm{~m}, 2 \mathrm{H}), 3.69$ (t, $J=4.9 \mathrm{~Hz}, 1 \mathrm{H}), 3.32(\mathrm{~s}, 3 \mathrm{H})$, $3.03(\mathrm{~d}, J=3.8 \mathrm{~Hz}, 1 \mathrm{H}), 1.93-1.86(\mathrm{~m}, 2 \mathrm{H}), 0.91(\mathrm{~s}, 9 \mathrm{H}), 0.10(\mathrm{~d}, J=1.1 \mathrm{~Hz}, 6 \mathrm{H})$. 


\section{E) Mannosides}

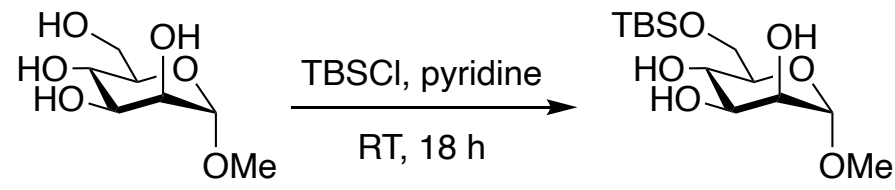

D-O6-TBS- $\alpha$-methylmannoside S8 was prepared according to an adapted procedure. See the synthesis of S5 for details. ${ }^{13}$ Obtained $0.524 \mathrm{~g}$ as a white solid, $1.70 \mathrm{mmol}, 85 \%$. ${ }^{1} \mathrm{H}$ NMR (400 MHz, MeOD) $\delta 4.61(\mathrm{~d}, J=1.6 \mathrm{~Hz}, 1 \mathrm{H}), 3.98(\mathrm{dd}, J=11.1,2.0 \mathrm{~Hz}, 1 \mathrm{H}), 3.85-3.71(\mathrm{~m}, 2 \mathrm{H}), 3.64$ $(\mathrm{dd}, J=8.8,3.4 \mathrm{~Hz}, 1 \mathrm{H}), 3.57-3.44(\mathrm{~m}, 2 \mathrm{H}), 3.36(\mathrm{~s}, 3 \mathrm{H}), 0.92(\mathrm{~s}, 9 \mathrm{H}), 0.10(\mathrm{~d}, J=1.4 \mathrm{~Hz}, 6 \mathrm{H})$.

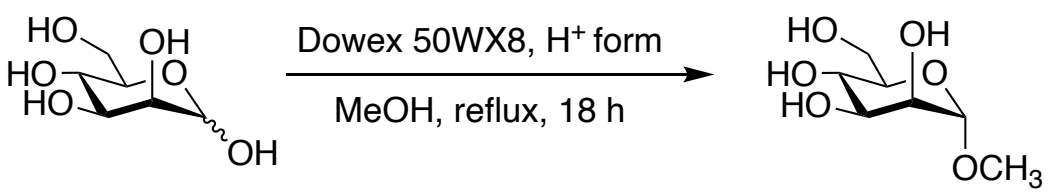

D- $\boldsymbol{\alpha}$-methylmannosides 6 and S9-S11 were prepared using standard Fisher glycosylation conditions. Mannose $(0.1 \mathrm{~g})$ and Dowex ${ }^{\mathrm{TM}}$ 50WX8-100 ion-exchange resin (0.2 $\left.\mathrm{g}\right)$ were added to a 2 dram vial equipped with a stir bar. The vial was capped with a septum cap and purged with nitrogen for $10 \mathrm{~min} .1 .5 \mathrm{~mL}$ anhydrous methanol was added via syringe. The reaction was stirred and heated at $65{ }^{\circ} \mathrm{C}$ for $18 \mathrm{~h}$. The reaction was cooled to room temperature. The resin was filtered and washed with $\mathrm{MeOH}$. The resulting filtrate was concentrated under reduced pressure. The resulting oil was recrystallized from hot methanol (0.5-1.0 mL). D- $\alpha$-methylmannosides 6 and S7S9 were obtained as white crystals $(\sim 66 \%,>20: 1 \alpha / \beta)$.

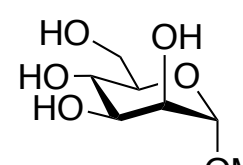

OMe D-a-methylmannoside 6: Prepared from unlabeled mannose and methanol as an analytical standard. ${ }^{1} \mathrm{H}$ NMR $(400 \mathrm{MHz}, \mathrm{MeOD}) \delta 4.63(\mathrm{~d}, J=1.7 \mathrm{~Hz}, 1 \mathrm{H}), 3.84(\mathrm{dd}, J=11.7,2.4$ $\mathrm{Hz}, 1 \mathrm{H}), 3.78(\mathrm{dd}, J=3.3,1.7 \mathrm{~Hz}, 1 \mathrm{H}), 3.71(\mathrm{dd}, J=11.8,5.9 \mathrm{~Hz}, 1 \mathrm{H}), 3.66(\mathrm{dd}, J=9.3,3.3 \mathrm{~Hz}$, $1 \mathrm{H}), 3.60(\mathrm{dd}, J=9.4 \mathrm{~Hz}, 1 \mathrm{H}), 3.48(\mathrm{ddd}, J=9.4,5.8,2.4 \mathrm{~Hz}, 1 \mathrm{H}), 3.38(\mathrm{~s}, 3 \mathrm{H}) .{ }^{13} \mathrm{C}$ NMR $(100$ MHz, MeOD) $\delta 102.73,74.48,72.60,72.09,68.60,62.93,55.19$. HRMS (DART) $\left([\mathrm{M}+\mathrm{H}]^{+}\right)$ Calcd. For C7H15O6: 195.08631. Found 195.08644. 


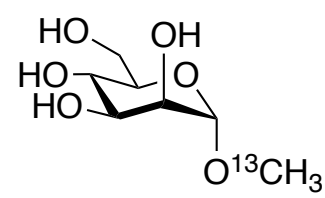

${ }_{3}$ D- $\alpha$-methylmannoside S9: Prepared from unlabeled mannose and ${ }^{13} \mathrm{C}$ labeled methanol. ${ }^{1} \mathrm{H}$ NMR (400 MHz, MeOD) $\delta 4.63(\mathrm{dd}, J=3.9,1.7 \mathrm{~Hz}, 1 \mathrm{H}), 3.84(\mathrm{dd}, J=11.8,2.4$ $\mathrm{Hz}, 1 \mathrm{H}), 3.78(\mathrm{dd}, J=3.3,1.7 \mathrm{~Hz}, 1 \mathrm{H}), 3.71(\mathrm{dd}, J=11.7,5.9 \mathrm{~Hz}, 1 \mathrm{H}), 3.66(\mathrm{dd}, J=9.4,3.3 \mathrm{~Hz}$, $1 \mathrm{H}), 3.60(\mathrm{t}, J=9.4 \mathrm{~Hz}, 1 \mathrm{H}), 3.51-3.44(\mathrm{~m}, 1 \mathrm{H}), 3.37$ (d, $J=142.5 \mathrm{~Hz}, 3 \mathrm{H}) .{ }^{13} \mathrm{C}$ NMR $(100 \mathrm{MHz}$, MeOD) $\delta 102.73(\mathrm{~d}, J=2.1 \mathrm{~Hz}), 74.48,72.60,72.09$ (d, $J=4.0 \mathrm{~Hz}), 68.60,62.93,55.19$. HRMS (DART) $\left.([\mathrm{M}+\mathrm{H}]]^{+}\right)$Calcd. For ${ }^{13} \mathrm{C} 1 \mathrm{C} 6 \mathrm{H} 15 \mathrm{O} 6: 196.08967$. Found 196.08939.

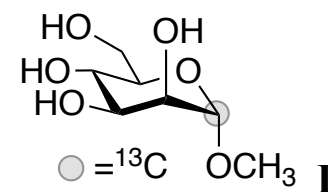

D-a-methylmannoside S10: Prepared from ${ }^{13} \mathrm{C}_{1}$-mannose and unlabeled methanol. ${ }^{1} \mathrm{H}$ NMR (400 MHz, MeOD) $\delta 4.63(\mathrm{dd}, J=168.4,1.5 \mathrm{~Hz}, 5 \mathrm{H}), 3.84$ (dd, $J=11.8,2.4$ $\mathrm{Hz}, 1 \mathrm{H}), 3.77$ (dt, $J=3.1,1.4 \mathrm{~Hz}, 1 \mathrm{H}), 3.71$ (dd, $J=11.7,5.9 \mathrm{~Hz}, 1 \mathrm{H}), 3.66$ (dd, $J=9.3,3.3 \mathrm{~Hz}$, 1H), $3.60(\mathrm{t}, J=9.4 \mathrm{~Hz}, 1 \mathrm{H}), 3.51-3.44(\mathrm{~m}, 1 \mathrm{H}), 3.38$ (d, $J=4.3 \mathrm{~Hz}, 3 \mathrm{H}) .{ }^{13} \mathrm{C}$ NMR $(100 \mathrm{MHz}$, MeOD) $\delta 102.73,74.48(\mathrm{~d}, J=2.1 \mathrm{~Hz}), 72.60,72.08(\mathrm{~d}, J=47.5 \mathrm{~Hz}), 68.60,62.93(\mathrm{~d}, J=3.4 \mathrm{~Hz})$, $55.18(\mathrm{~d}, J=2.1 \mathrm{~Hz})$. HRMS (DART) $\left.([\mathrm{M}+\mathrm{H}]]^{+}\right)$Calcd. For ${ }^{13} \mathrm{C} 1 \mathrm{C} 6 \mathrm{H} 15 \mathrm{O}$ : 196.08967. Found 196.08969 .

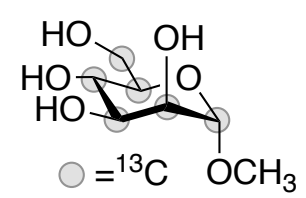

D-a-methylmannoside S11: Prepared from ${ }^{13} \mathrm{C}_{1-6}$-mannose and unlabeled methanol. The ${ }^{1} \mathrm{H}$ NMR (400 MHz, MeOD) is not interpretable. ${ }^{13} \mathrm{C}$ NMR (100 MHz, MeOD) $\delta$ $102.72(\mathrm{dd}, J=44.2,3.1 \mathrm{~Hz}), 75.29-73.63(\mathrm{~m}), 73.22-72.18(\mathrm{~m}), 72.52-71.41(\mathrm{~m}), 69.10$ $67.96(\mathrm{~m}), 62.92(\mathrm{dt}, J=43.3,3.4 \mathrm{~Hz}), 55.18(\mathrm{~m})$. HRMS (DART) $\left([\mathrm{M}+\mathrm{H}]^{+}\right)$Calcd. For ${ }^{13} \mathrm{C} 6 \mathrm{C} 1 \mathrm{H} 15 \mathrm{O6}: 201.10644$. Found 201.10680. 


\section{General Reaction Procedure of Redox Isomerization}

\section{General Procedure A) Procedure for Condition Optimization (0.1 mmol Rxn Scale)}

Into a 1-dram vial, L- $\alpha$-methylrhamnoside 1 (17.8 mg, $0.1 \mathrm{mmol}$ ), 4CzIPN (1.6 mg, $2 \mathrm{~mol} \%$ ), quinuclidine (1.1 mg, $10 \mathrm{~mol} \%), \mathrm{Mn}(\mathrm{OAc})_{2} \bullet 4 \mathrm{H}_{2} \mathrm{O}(2.5 \mathrm{mg}, 10 \mathrm{~mol} \%)$, and $\mathrm{Bu}_{4} \mathrm{NOAc}(1.5 \mathrm{mg}$, $5 \mathrm{~mol} \%$ ) were subsequently added. A 10x3 mm PTFE magnetic stir bar was added, and the vial was slightly capped and transferred into a nitrogen-filled purge box. Dry and degassed 5\% DMSO in $\mathrm{MeCN}(0.5 \mathrm{~mL})$ was added. The vial was then capped with a polypropylene screw cap with a bonded PTFE faced silicone liner and then removed from the purge box. The reaction vessel was then placed $5 \mathrm{~cm}$ from a blue LED Kessil lamp and stirred at $300 \mathrm{rpm}$ with a cooling fan. After 18 hours, the crude reaction mixture was concentrated and analyzed by ${ }^{1} \mathrm{H}$ NMR spectroscopy with nitrobenzene as analytical standard.

\section{General Procedure B) Procedure for Substrate Scope (0.3 mmol Rxn Scale)}

Into a 2-dram vial, L- $\alpha$-methylrhamnoside 1 ( $53.5 \mathrm{mg}, 0.3 \mathrm{mmol}$ ), 4CzIPN (4.7 mg, $2 \mathrm{~mol} \%$ ), quinuclidine (1.7 mg, $5 \mathrm{~mol} \%), \mathrm{Mn}(\mathrm{OAc})_{2} \bullet 4 \mathrm{H}_{2} \mathrm{O}$ (7.4 mg, $10 \mathrm{~mol} \%$ ), and $\mathrm{Bu} 4 \mathrm{NOAc}(9.0 \mathrm{mg}, 5$ mol \%) were subsequently added. A PTFE magnetic stir bar was added, and the vial was slightly capped and transferred into a nitrogen-filled purge box. Dry and degassed 5\% DMSO in MeCN $(1.5 \mathrm{~mL})$ was added. The vial was then capped with a polypropylene screw cap with a bonded PTFE faced silicone liner and then removed from the purge box. The reaction vessel was then placed $5 \mathrm{~cm}$ from 2x blue LED Kessil lamps and stirred at $500 \mathrm{rpm}$ with cooling fans and water bath (similar setup as shown in Figure S1, except with a smaller beaker). After 18 hours, the crude reaction mixture was concentrated and analyzed by ${ }^{1} \mathrm{H}$ NMR spectroscopy with nitrobenzene as analytical standard.

\section{General Procedure C) Procedure for Gram-Scale Reactions (6.0 mmol Rxn Scale)}

Into a $40 \mathrm{~mL}$ vial, L- $\alpha$-methylrhamnoside 1 (1069 mg, 6 mmol), 4CzIPN (95 mg, $2 \mathrm{~mol} \%$ ), quinuclidine (33 mg, $5 \mathrm{~mol} \%$ ), $\mathrm{Mn}(\mathrm{OAc})_{2} \bullet 4 \mathrm{H}_{2} \mathrm{O}$ (147 mg, $10 \mathrm{~mol} \%$ ), and $\mathrm{Bu}_{4} \mathrm{NOAc}$ (90 mg, 5 mol \%) were subsequently added. A PTFE magnetic stir bar was added, and the vial was slightly capped and transferred into a nitrogen-filled purge box. Dry and degassed 5\% DMSO in MeCN $(30 \mathrm{~mL})$ was added. The vial was then capped with a polypropylene screw cap with a bonded PTFE faced silicone liner and then removed from the purge box. The reaction vessel was then placed 5 
$\mathrm{cm}$ from $2 \mathrm{x}$ blue LED Kessil lamps and stirred at $500 \mathrm{rpm}$ with cooling fans and water bath (shown in Figure S1). After 18 hours, the crude reaction mixture was concentrated and analyzed by ${ }^{1} \mathrm{H}$ NMR spectroscopy with nitrobenzene as analytical standard.

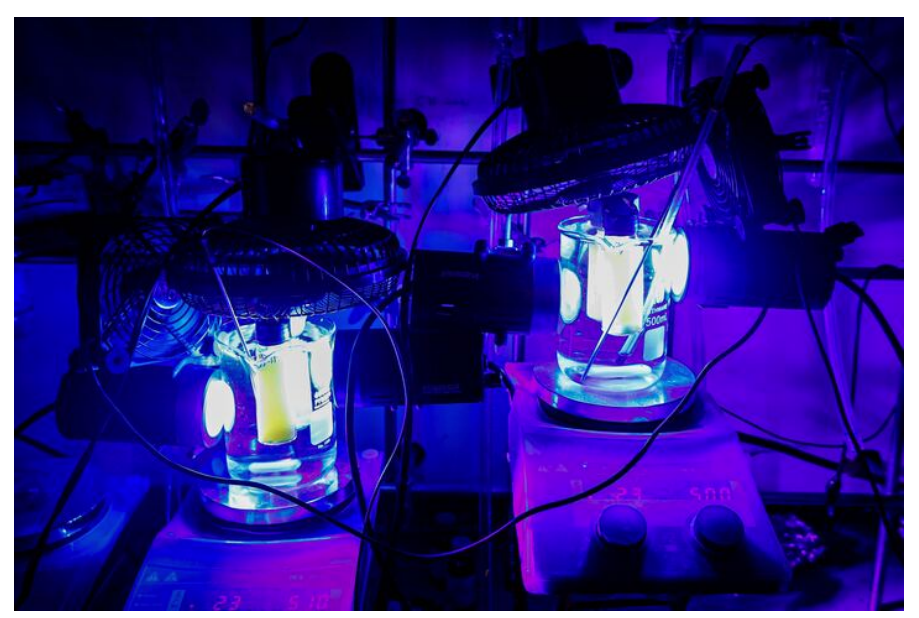

Figure S1. Reaction setup for $6 \mathrm{mmol}$ scale reaction with 2 Kessils, water bath, and two fans.

\section{D) Notes on reaction setup, purification, and analysis}

- The particular wavelength of blue LED Kessils was found to have no effect (427 - 456 $\mathrm{nm})$.

- Water baths were used at larger scales to avoid excessive heating. The water evaporated overnight, but this found to have no effect on reaction outcome.

- Avoid diluting the reaction with methanol as excessive exposure to polar protic solvents resulted in product degradation.

- Crude reaction NMRs were taken in MeOD to avoid broadening caused by $\mathrm{Mn}(\mathrm{II})$; concentration $\sim 2-3$ drop (pipette tip) in $500 \mu \mathrm{L} \mathrm{MeOD}$.

- Upon concentrating the reaction mixture, they were immediately columned or stored in the fridge for less than 2 days before chromatography. 


\section{E) Product Characterization}

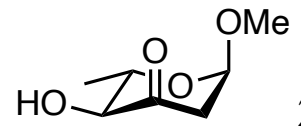

2: Prepared according to general procedure B using L- $\alpha$-methyl rhamnoside $(0.3$ mmol). The reaction mixture was purified by column chromatography (EtOAc/DCM) to give 2 (22 mg, 45\%).

2 was also prepared according to general procedure C using L- $\alpha$-methyl rhamnoside (6.0 mmol).

The reaction mixture was purified by column chromatography (EtOAc/DCM) to give 2 (432 mg, $45 \%)$.

10:1 DCM/EtOAc, $\mathrm{R}_{\mathrm{f}}=0.61 .{ }^{1} \mathrm{H}$ NMR $\left(400 \mathrm{MHz}, \mathrm{CDCl}_{3}\right) \delta 5.09(\mathrm{~d}, J=4.4 \mathrm{~Hz}, 1 \mathrm{H}), 3.86-3.72$ (m, 2H), 3.55 (s, 1H), 3.34 (s, 3H), $2.81(\mathrm{ddd}, J=14.1,4.7,1.4 \mathrm{~Hz}, 1 \mathrm{H}), 2.68$ (dd, $J=14.0,1.1$ $\mathrm{Hz}, 1 \mathrm{H}), 1.45(\mathrm{~d}, J=5.9 \mathrm{~Hz}, 3 \mathrm{H}) .{ }^{13} \mathrm{C} \mathrm{NMR}\left(100 \mathrm{MHz}, \mathrm{CDCl}_{3}\right) \delta 205.39,99.73,78.64,71.05$, $54.96,45.34,18.86$.

The NMR spectra are consistent with those reported in the literature. ${ }^{14}$

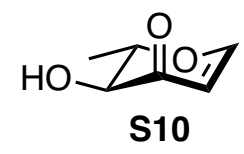

Enone, S10, is commonly observed post chromatography in small amounts $(<3 \%) .{ }^{14}$

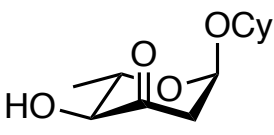

4: Prepared according to general procedure B using L- $\alpha$-cyclohexyl rhamnoside $(0.3 \mathrm{mmol})$. The reaction mixture was purified by column chromatography (EtOAc/DCM) to give $4(25 \mathrm{mg}, 37 \%)$.

10:1 DCM/EtOAc, $\mathrm{R}_{\mathrm{f}}=0.69 .[\alpha]^{20}{ }_{\mathrm{D}}=-120.3^{\circ}(\mathrm{c}=1, \mathrm{MeOH}) .{ }^{1} \mathrm{H}$ NMR $\left(400 \mathrm{MHz}, \mathrm{CDCl}_{3}\right) \delta 5.34$ $(\mathrm{d}, J=4.5 \mathrm{~Hz}, 1 \mathrm{H}), 3.91-3.76(\mathrm{~m}, 2 \mathrm{H}), 3.55(\mathrm{tt}, J=9.1,3.7 \mathrm{~Hz}, 2 \mathrm{H}), 2.80(\mathrm{ddd}, J=13.9,4.8,1.4$ $\mathrm{Hz}, 1 \mathrm{H}), 2.61(\mathrm{dd}, J=13.8,1.1 \mathrm{~Hz}, 1 \mathrm{H}), 1.89-1.62(\mathrm{~m}, 4 \mathrm{H}), 1.57-1.44(\mathrm{~m}, 1 \mathrm{H}), 1.41(\mathrm{~d}, J=5.9$ $\mathrm{Hz}, 3 \mathrm{H}), 1.37-1.13(\mathrm{~m}, 5 \mathrm{H}) .{ }^{13} \mathrm{C} \mathrm{NMR}\left(100 \mathrm{MHz}, \mathrm{CDCl}_{3}\right) \delta 205.74,96.42,78.68,75.23,71.13$, 45.87, 33.32, 31.35, 25.66, 24.20, 23.94, 18.79. IR (neat, $\mathrm{cm}^{-1}$ ): 3458.9 (br), 2933.4 (s), 2858.9 (m), 1725.8 (s), 1449.9 (w), 1118.2 (s). HRMS (DART) $\left([\mathrm{M}+\mathrm{H}]^{+}\right)$Calcd. For C12H21O4: 229.14344 , found 229.14250 . 
Ho Tlloj

5: Prepared according to general procedure B using L- $\alpha$-cyclobenzyl rhamnoside $(0.3 \mathrm{mmol})$. The reaction mixture was purified by column chromatography (EtOAc/DCM) to give 5 (28 mg, 39\%).

10:1 DCM/EtOAc, $\mathrm{R}_{\mathrm{f}}=0.75 .[\alpha]^{20} \mathrm{D}=-96.6^{\circ}(\mathrm{c}=1, \mathrm{MeOH}) .{ }^{1} \mathrm{H}$ NMR $\left(400 \mathrm{MHz}, \mathrm{CDCl}_{3}\right) \delta 7.39$ - $7.27(\mathrm{~m}, 5 \mathrm{H}), 5.27(\mathrm{~d}, J=4.8 \mathrm{~Hz}, 1 \mathrm{H}), 4.72-4.64(\mathrm{~m}, 1 \mathrm{H}), 4.50$ (d, J=12.1 Hz, 1H), 3.88 $3.82(\mathrm{~m}, 2 \mathrm{H}), 3.57(\mathrm{~s}, 1 \mathrm{H}), 2.82(\mathrm{dd}, J=14.1,4.7 \mathrm{~Hz}, 1 \mathrm{H}), 2.71(\mathrm{dd}, J=14.1,1.1 \mathrm{~Hz}, 1 \mathrm{H}), 1.47-$ $1.42(\mathrm{~m}, 3 \mathrm{H}) .{ }^{13} \mathrm{C}$ NMR $\left(100 \mathrm{MHz}, \mathrm{CDCl}_{3}\right) \delta 205.35,136.95,128.61,128.07,128.01,97.64,78.59$, 71.36, 69.06, 45.25, 18.85. IR (neat, $\mathrm{cm}^{-1}$ ): 3451.5 (br), 2933.4 (br), 1725.8 (s), 1453.6 (w), 1121.9 (s), $738.0(\mathrm{~m})$. HRMS (DART) $\left([\mathrm{M}+\mathrm{H}]^{+}\right)$Calcd. For C13H17O4: 237.11214, found 237.11253.

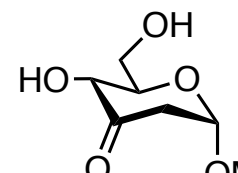

OMe 7: Prepared according to general procedure B using D- $\alpha$-methyl mannoside $(0.3$ mmol), 20\% DMSO, and $10 \mathrm{~mol} \% \mathrm{KOBz}$ used instead of $5 \mathrm{~mol} \% \mathrm{Bu}_{4} \mathrm{NOAc}$. The reaction mixture was purified by column chromatography with C2-deactivated silica (MeOH/DCM) to give 7. A satisfactory isolated yield could not be obtained to degradation on silica, including solvent mixtures without $\mathrm{MeOH}$. Small amounts of clean compound 7, could be isolated for characterization.

20:1 DCM/MeOH, $\mathrm{R}_{\mathrm{f}}=0.375 .[\alpha]^{20} \mathrm{D}=171.3(\mathrm{c}=1, \mathrm{MeOH}) .{ }^{1} \mathrm{H}$ NMR $(400 \mathrm{MHz}, \mathrm{MeOD}) \delta 5.14$ $(\mathrm{d}, J=4.4 \mathrm{~Hz}, 1 \mathrm{H}), 4.17$ (d, $J=10.0 \mathrm{~Hz}, 1 \mathrm{H}), 3.84(\mathrm{qd}, J=12.0,3.6 \mathrm{~Hz}, 2 \mathrm{H}), 3.69$ (ddd, $J=9.9$, 4.7, $2.3 \mathrm{~Hz}, 1 \mathrm{H}), 3.34(\mathrm{~s}, 3 \mathrm{H}), 2.88(\mathrm{dd}, J=13.5,3.9 \mathrm{~Hz}, 1 \mathrm{H}), 2.50(\mathrm{~d}, J=14.2 \mathrm{~Hz}, 1 \mathrm{H}) .{ }^{13} \mathrm{C} \mathrm{NMR}$ (100 MHz, MeOD) $\delta 207.25,101.19,76.38,74.12,62.64,55.02,46.65$. IR (neat, $\left.\mathrm{cm}^{-1}\right): 3440.3$ (br), 2922.2 (br), 1722.0 (s), 1364.2 (w), 1095.8 (s), 1036.2 (s), 954.2 (w). HRMS (DART) $\left([\mathrm{M}+\mathrm{H}]^{+}\right)$Calcd. For C7H13O5: 177.07575. Found 177.07538. 


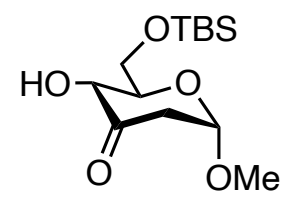

8: Prepared according to general procedure $B$ using O6-TBS-D- $\alpha$-methyl mannoside (0.3 mmol), 10\% DMSO, and $10 \mathrm{~mol} \% \mathrm{KOBz}$ used instead of $5 \mathrm{~mol} \% \mathrm{Bu} 4 \mathrm{NOAc}$. The reaction mixture was purified by column chromatography (EtOAc/Hex) to give $\mathbf{8}$ (28 $\mathrm{mg}$, $36 \%)$.

10:1 DCM/EtOAc, $\mathrm{R}_{\mathrm{f}}=0.78 .[\alpha]^{20}{ }_{\mathrm{D}}=103.8^{\circ}(\mathrm{c}=1, \mathrm{MeOH}) .{ }^{1} \mathrm{H}$ NMR $\left(400 \mathrm{MHz}, \mathrm{CDCl}_{3}\right) \delta 5.16$ (d, $J=4.6 \mathrm{~Hz}, 1 \mathrm{H}), 4.18$ (dd, $J=9.7,2.7 \mathrm{~Hz}, 1 \mathrm{H}), 4.03-3.89$ (m, 2H), 3.67 (ddd, $J=9.9,4.5,2.3$ Hz, 1H), $3.54(\mathrm{~d}, J=3.9 \mathrm{~Hz}, 1 \mathrm{H}), 3.34(\mathrm{~s}, 3 \mathrm{H}), 2.80(\mathrm{dd}, J=14.1,4.6 \mathrm{~Hz}, 1 \mathrm{H}), 2.67(\mathrm{~d}, J=12.9$ $\mathrm{Hz}, 1 \mathrm{H}), 0.92$ (s, 9H), 0.11 (s, 6H). ${ }^{13} \mathrm{C}$ NMR (100 MHz, $\left.\mathrm{CDCl}_{3}\right) \delta$ 206.06, 99.86, 75.59, 73.10, 62.96, 54.86, 45.24, 26.11, 18.61, -5.13. IR (neat, $\mathrm{cm}^{-1}$ ): 3466.4 (br), 2929.7 (br), 2858.9 (m), 1729.5 (s), 1461.1 (w), 1103.3 (s), 834.9 (w). HRMS (DART) ([M+H] $\left.]^{+}\right)$Calcd. For C13H27O5Si: 291.16223, found 291.16196.

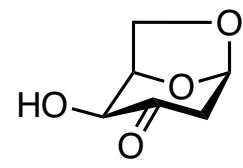

10: Prepared according to general procedure $B$ using $D$-anhydrogalactose $(0.3$ $\mathrm{mmol}), 10 \mathrm{~mol} \% \mathrm{KOBz}$ used instead of $5 \mathrm{~mol} \% \mathrm{Bu}_{4} \mathrm{NOAc}$. The reaction mixture was purified by column chromatography (Hex/EtOAc) to give $\mathbf{1 0}(21 \mathrm{mg}, 48 \%)$.

1:1 DCM/EtOAc, $\mathrm{R}_{\mathrm{f}}=0.73 .[\alpha]^{20}{ }_{\mathrm{D}}=-82.3(\mathrm{c}=1, \mathrm{MeOH}) .{ }^{1} \mathrm{H}$ NMR $(400 \mathrm{MHz}, \mathrm{MeOD}) \delta 5.77(\mathrm{~s}$, $1 \mathrm{H}), 4.75(\mathrm{t}, J=5.1 \mathrm{~Hz}, 1 \mathrm{H}), 4.35(\mathrm{~d}, J=5.4 \mathrm{~Hz}, 1 \mathrm{H}), 3.96(\mathrm{~d}, J=8.0 \mathrm{~Hz}, 1 \mathrm{H}), 3.76-3.68(\mathrm{~m}$, $1 \mathrm{H}), 2.77(\mathrm{~d}, J=15.5 \mathrm{~Hz}, 1 \mathrm{H}), 2.55(\mathrm{~d}, J=15.5 \mathrm{~Hz}, 1 \mathrm{H}) .{ }^{13} \mathrm{C}$ NMR $(100 \mathrm{MHz}, \mathrm{MeOD}) \delta 206.99$, 102.96, 77.88, 76.50, 66.59, 49.28. IR (neat, $\mathrm{cm}^{-1}$ ): 3444.1 (br), 2966.9 (w), 2907.3 (w), 1725.7 (s), $1211.4(\mathrm{w}), 1125.6(\mathrm{~s}), 861.0(\mathrm{~m})$. HRMS (DART) $\left([\mathrm{M}+\mathrm{H}]^{+}\right)$Calcd. For C6H9O4: 145.04954, found 145.05000 . 


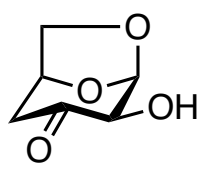

12: Prepared according to general procedure $B$ using $D$-anhydromannose $(0.3 \mathrm{mmol})$, $10 \mathrm{~mol} \% \mathrm{KOBz}$ used instead of $5 \mathrm{~mol} \% \mathrm{Bu} 4 \mathrm{NOAc}$. The reaction mixture was purified by column chromatography (Hex/EtOAc) to give 12 (22 mg, 52\%).

1:1 DCM/EtOAc, $\mathrm{R}_{\mathrm{f}}=0.62 .[\alpha]^{20}{ }_{\mathrm{D}}=-104.9(\mathrm{c}=1, \mathrm{MeOH}) .{ }^{1} \mathrm{H}$ NMR $(400 \mathrm{MHz}, \mathrm{MeOD}) \delta 5.54$ (d, $J=2.5 \mathrm{~Hz}, 1 \mathrm{H}), 4.89$ (t, $J=4.9 \mathrm{~Hz}, 1 \mathrm{H}), 4.10(\mathrm{~s}, 1 \mathrm{H}), 3.76$ (dt, $J=13.1,4.8 \mathrm{~Hz}, 2 \mathrm{H}), 2.91$ (dd, $J=15.6,5.1 \mathrm{~Hz}, 1 \mathrm{H}), 2.49$ (d, $J=15.5 \mathrm{~Hz}, 1 \mathrm{H}) .{ }^{13} \mathrm{C} \mathrm{NMR}(100 \mathrm{MHz}, \mathrm{MeOD}) \delta 207.08,104.84$, 78.54, 75.26, 70.62, 47.12. IR (neat, $\mathrm{cm}^{-1}$ ): 3444.1 (br), 2966.9 (w), 2907.3 (w), 1725.7 (s), 1326.9 (w), $1095.8(\mathrm{~s}), 1013.8(\mathrm{~m})$. HRMS (DART) $\left([\mathrm{M}+\mathrm{H}]^{+}\right)$Calcd. For C6H9O4: 145.04954, found 145.05002 .

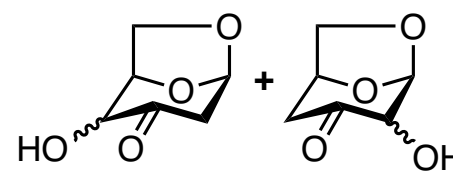

15 and 16: Prepared according to general procedure $B$ using Danhydroglucose $(0.3 \mathrm{mmol}), 10 \mathrm{~mol} \% \mathrm{KOBz}$ used instead of $5 \mathrm{~mol} \% \mathrm{Bu} 4 \mathrm{NOAc}$. The reaction mixture was purified by column chromatography (Hex/EtOAc). No separation of C2-epi-10 and C4-epi-12 was observed. C2-epi-10 and C4-epi-12 could not be isolated in sufficient quantity and purity for full characterization. A stack plot of ${ }^{1} \mathrm{H}$ NMRs $(\mathbf{1 0}, 12$, crude reaction of anhydroglucose, and a purified fraction) is presented in the spectra section. 


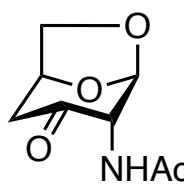

18: Prepared according to general procedure $B$ using D-anhydroglucosamine $(0.3$ $\mathrm{mmol})$. The reaction mixture was purified by column chromatography (MeOH/DCM). The product isomerized on silica, however small amounts of clean compound, 18, could be isolated for characterization.

20:1 DCM/MeOH, $\mathrm{R}_{\mathrm{f}}=0.58 .[\alpha]^{20}{ }_{\mathrm{D}}=-140.28(\mathrm{c}=1, \mathrm{MeOH}) .{ }^{1} \mathrm{H}$ NMR $\left(400 \mathrm{MHz}, \mathrm{CDCl}_{3}\right) \delta 6.22$ (s, 1H), $5.76(\mathrm{~d}, J=1.9 \mathrm{~Hz}, 1 \mathrm{H}), 4.93(\mathrm{t}, J=4.9 \mathrm{~Hz}, 1 \mathrm{H}), 4.66$ (dt, $J=7.1,1.5 \mathrm{~Hz}, 1 \mathrm{H}), 3.84$ (ddd, $J=6.9,4.7,2.0 \mathrm{~Hz}, 1 \mathrm{H}), 3.79$ (d, $J=7.6 \mathrm{~Hz}, 1 \mathrm{H}), 2.93$ (ddt, $J=15.4,4.9,1.6 \mathrm{~Hz}, 1 \mathrm{H}), 2.57$ (dd, $J=15.3,1.4 \mathrm{~Hz}, 1 \mathrm{H}), 2.06(\mathrm{~s}, 3 \mathrm{H}) .{ }^{13} \mathrm{C} \mathrm{NMR}\left(100 \mathrm{MHz}, \mathrm{CDCl}_{3}\right) \delta 201.85,170.45,102.38,74.03$, 69.71, 62.37, 46.82, 23.17. IR (neat, $\left.\mathrm{cm}^{-1}\right): 3358.3$ (br), 1729.5 (s), 1662.4 (s), 1531.9 (m), 1375.4 (m), $1129.4(\mathrm{~s}), 976.5(\mathrm{~m})$. HRMS (DART) $\left([\mathrm{M}+\mathrm{H}]^{+}\right)$Calcd. For C8H12NO4: 186.07608, found 186.07570 .

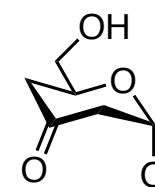

OMe 21: Prepared according to general procedure B using D- $\alpha$-methyl-2-deoxygalactoside $(0.3 \mathrm{mmol})$. The reaction mixture was purified by column chromatography (MeOH/DCM). A satisfactory isolated yield could not be obtained to degradation on silica, including solvent mixtures without $\mathrm{MeOH}$. Small amounts of clean compound, 21, could be isolated for characterization.

20:1 DCM/MeOH, $\mathrm{R}_{\mathrm{f}}=0.41 .[\alpha]^{20}{ }_{\mathrm{D}}=117.8(\mathrm{c}=1, \mathrm{MeOH}) .{ }^{1} \mathrm{H}$ NMR $\left(400 \mathrm{MHz}, \mathrm{CDCl}_{3}\right) \delta 5.17$ (d, $J=4.6 \mathrm{~Hz}, 1 \mathrm{H}), 4.14$ (ddt, $J=11.5,5.6,2.9 \mathrm{~Hz}, 1 \mathrm{H}), 3.81$ (dq, $J=11.9,2.9 \mathrm{~Hz}, 1 \mathrm{H}), 3.63$ (dt, $J=11.7,5.8 \mathrm{~Hz}, 1 \mathrm{H}), 3.37(\mathrm{~s}, 3 \mathrm{H}), 2.69-2.43(\mathrm{~m}, 3 \mathrm{H}), 2.38-2.27(\mathrm{~m}, 1 \mathrm{H}), 1.96(\mathrm{t}, J=6.4 \mathrm{~Hz}$, 1H). ${ }^{13} \mathrm{C}$ NMR $\left(100 \mathrm{MHz}, \mathrm{CDCl}_{3}\right) \delta 204.28,99.68,68.96,64.99,54.88,46.32,42.79$. IR (neat, $\mathrm{cm}^{-1}$ ): 3432.8 (br), 2914.7 (br), 1718.3 (s), 1628.8 (m), 1293.4 (w), 1043.6 (s). HRMS (DART) $\left([\mathrm{M}+\mathrm{H}]^{+}\right)$Calcd. For C7H13O4: 161.08084, found 161.08066. 


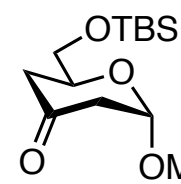

OMe 22: Prepared according to general procedure B using O6-TBS-D- $\alpha$-methyl-2-deoxygalactoside $(0.3 \mathrm{mmol})$. The reaction mixture was purified by column chromatography (EtOAc/Hex) to give 22 (43 mg, 52\%).

10:1 Hex/EtOAc, $\mathrm{R}_{\mathrm{f}}=0.37 .[\alpha]^{20} \mathrm{D}=76.3^{\circ}(\mathrm{c}=1, \mathrm{MeOH}) .{ }^{1} \mathrm{H}$ NMR $\left(400 \mathrm{MHz}, \mathrm{CDCl}_{3}\right) \delta 5.14(\mathrm{~d}$, $J=4.6 \mathrm{~Hz}, 1 \mathrm{H}), 4.12-4.02(\mathrm{~m}, 1 \mathrm{H}), 3.73(\mathrm{~d}, J=4.8 \mathrm{~Hz}, 2 \mathrm{H}), 3.35(\mathrm{~s}, 3 \mathrm{H}), 2.63$ (dd, $J=15.4,5.0$ $\mathrm{Hz}, 1 \mathrm{H}), 2.51-2.33(\mathrm{~m}, 3 \mathrm{H}), 0.90(\mathrm{~s}, 9 \mathrm{H}), 0.08(\mathrm{~s}, 6 \mathrm{H}) .{ }^{13} \mathrm{C} \mathrm{NMR}\left(100 \mathrm{MHz}, \mathrm{CDCl}_{3}\right) \delta 205.12$, 99.62, 69.44, 65.79, 54.85, 46.53, 43.64, 26.01, 18.50, -5.18, -5.20. IR (neat, cm ${ }^{-1}$ ): 2929.7 (br), $2858.9(\mathrm{~m}), 1729.5(\mathrm{~s}), 1472.3(\mathrm{w}), 1095.8(\mathrm{~s}), 834.9(\mathrm{~s})$. HRMS (DART) $\left([\mathrm{M}+\mathrm{H}]^{+}\right)$Calcd. For C13 H27 O4 Si : 275.16731. Found 275.16746.

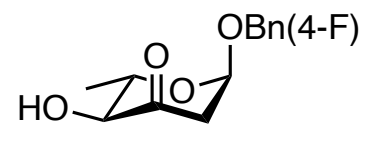

S12: Prepared according to general procedure B using D- $\alpha-(4-F)$ benzylrhamnoside $(0.3 \mathrm{mmol})$. The reaction mixture was purified by column chromatography (Hex/EtOAc) to give S12 (31 mg, 40\%).

3:1 Hex/EtOAc, $\mathrm{R}_{\mathrm{f}}=0.28 .[\alpha]^{20}{ }_{\mathrm{D}}=-105.2(\mathrm{c}=1, \mathrm{MeCN}) .{ }^{1} \mathrm{H} \mathrm{NMR}\left(400 \mathrm{MHz}, \mathrm{CD}_{3} \mathrm{CN}\right) \delta 7.39-$ $7.30(\mathrm{~m}, 2 \mathrm{H}), 7.18-7.04(\mathrm{~m}, 2 \mathrm{H}), 5.28(\mathrm{dt}, J=4.6,0.7 \mathrm{~Hz}, 1 \mathrm{H}), 4.61(\mathrm{~d}, J=11.7 \mathrm{~Hz}, 1 \mathrm{H}), 4.45$ $(\mathrm{d}, J=11.7 \mathrm{~Hz}, 1 \mathrm{H}), 3.84(\mathrm{ddd}, J=9.6,4.5,1.3 \mathrm{~Hz}, 1 \mathrm{H}), 3.75(\mathrm{dq}, J=9.6,6.0 \mathrm{~Hz}, 1 \mathrm{H}), 3.62(\mathrm{~d}, J$ $=4.5 \mathrm{~Hz}, 1 \mathrm{H}), 2.89(\mathrm{ddd}, J=14.2,4.7,1.3 \mathrm{~Hz}, 1 \mathrm{H}), 2.50(\mathrm{dd}, J=14.2,1.1 \mathrm{~Hz}, 1 \mathrm{H}), 1.36(\mathrm{~d}, J=$ $6.0 \mathrm{~Hz}, 3 \mathrm{H}) .{ }^{13} \mathrm{C} \mathrm{NMR}\left(100 \mathrm{MHz}, \mathrm{CDCl}_{3}\right) \delta 205.25,162.63(\mathrm{~d}, J=246.3 \mathrm{~Hz}), 132.73(\mathrm{~d}, J=3.2$ $\mathrm{Hz}), 129.82(\mathrm{~d}, J=8.2 \mathrm{~Hz}), 115.52(\mathrm{~d}, J=21.5 \mathrm{~Hz}), 97.63,78.57,71.45,68.40,45.23,18.86 .{ }^{19} \mathrm{~F}$ NMR (376 MHz, $\left.\mathrm{CD}_{3} \mathrm{CN}\right) \delta-117.21$ (ddd, $J=14.6,9.2,5.7 \mathrm{~Hz}$ ). IR (neat, $\mathrm{cm}^{-1}$ ): 3459.0 (br, w), 2933.4 (br, w), 1725.8 (s), 1509.6 (s), 1222.6 (s), 1118.2 (s), 767.8 (m). HRMS (DART) $\left(\left[\mathrm{M}+\mathrm{NH}_{4}\right]^{+}\right)$Calcd. For C13H19NO4F: 272.12926, found 272.13018. 


\section{Derivatizations of Products of Redox Isomerization}

\section{A) Cis-selective Ketone Reduction}

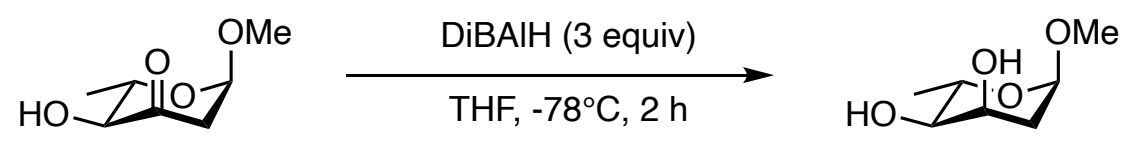

Into a round bottom flask, sugar $2(100 \mathrm{mg}, 0.624 \mathrm{mmol})$ and $6 \mathrm{~mL}$ of anhydrous THF was added with stir bar. The mixture was cooled to $-78^{\circ} \mathrm{C}$. DIBAl-H (1 M in hexanes) $(1.87 \mathrm{~mL}, 1.87 \mathrm{mmol})$ was added dropwise over $10 \mathrm{~min}$. The resulting mixture was let stir at $-78{ }^{\circ} \mathrm{C}$ for $2 \mathrm{~h}$. The reaction was then brought to $0{ }^{\circ} \mathrm{C}$, diluted with ethyl acetate and worked up with the Lou Fieser workup. $74 \mu \mathrm{L}$ of $\mathrm{H}_{2} \mathrm{O}$ was added dropwise, then $74 \mu \mathrm{L}$ of $15 \% \mathrm{NaOH}$, and then $190 \mu \mathrm{L} \mathrm{H}_{2} \mathrm{O}$. The mixture was warmed to room temperature, let stir for $15 \mathrm{~min}$ and then magnesium sulfate was added and let stir for another $15 \mathrm{~min}$. The mixture was filtered and concentrated under reduced pressure. The reaction mixture was purified by column chromatography (MeOH/DCM) to give $\mathbf{2 3}$ as a clear oil (75 mg, 74\%).

1:1 EA/Hex, $\mathrm{R}_{\mathrm{f}}=0.34 .{ }^{1} \mathrm{H} \mathrm{NMR}\left(400 \mathrm{MHz}, \mathrm{CDCl}_{3}\right) \delta 4.73(\mathrm{~d}, J=4.1 \mathrm{~Hz}, 1 \mathrm{H}), 3.90(\mathrm{dd}, J=10.0$, $3.4 \mathrm{~Hz}, 1 \mathrm{H}), 3.68(\mathrm{dq}, J=9.9,6.1 \mathrm{~Hz}, 1 \mathrm{H}), 3.42(\mathrm{~d}, J=10.0 \mathrm{~Hz}, 1 \mathrm{H}), 3.34(\mathrm{~s}, 3 \mathrm{H}), 3.11(\mathrm{td}, J=$ 10.0, 3.6 Hz, 1H), 2.66 (d, $J=10.7 \mathrm{~Hz}, 1 \mathrm{H}), 2.13$ (dd, $J=14.7,3.2 \mathrm{~Hz}, 1 \mathrm{H}), 1.87$ (dt, $J=14.7,3.5$ $\mathrm{Hz}, 1 \mathrm{H}), 1.30(\mathrm{~d}, J=6.3 \mathrm{~Hz}, 3 \mathrm{H}) .{ }^{13} \mathrm{C} \mathrm{NMR}\left(100 \mathrm{MHz}, \mathrm{CDCl}_{3}\right) \delta$ 98.42, 72.61, 67.51, 64.38, $55.21,35.19,17.89$.

The NMR spectra are consistent with those reported in the literature. ${ }^{15}$ 


\section{B) Trans-selective Ketone Reduction}

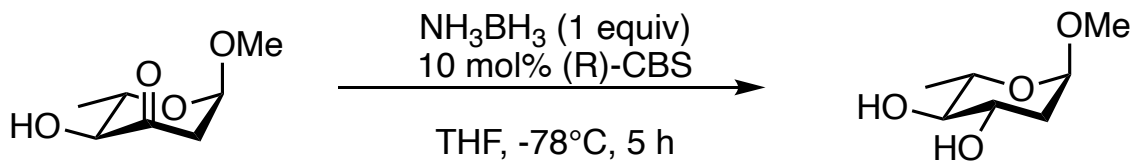

Into a round bottom flask, sugar $2(100 \mathrm{mg}, 0.624 \mathrm{mmol})$ and $6 \mathrm{~mL}$ of anhydrous THF was added with stir bar. In a separate round bottom flask, $\mathrm{NH}_{3} \mathrm{BH}_{3}(19.27 \mathrm{mg}, 0.624 \mathrm{mmol})$ and $(R)-(+)-o-$ Tolyl-CBS-oxazaborolidine solution $(0.5 \mathrm{M}$ in toluene) $(62.4 \mu \mathrm{L}, 0.0624 \mathrm{mmol})$ was added. Both were cooled to $-78^{\circ} \mathrm{C}$. The sugar mixture was then added dropwise to the borane mixture, and the resulting mixture was let stir at $-78^{\circ} \mathrm{C}$ for $5 \mathrm{~h}$. The reaction was brought to room temperature and quenched with $\mathrm{MeOH}(6 \mathrm{~mL}) .200 \mu \mathrm{L}$ of $1 \mathrm{M} \mathrm{HCl}$ was then added and the mixture was let stir overnight to hydrolyze the $\mathrm{O}-\mathrm{B}$ bond. The mixture was dried over $\mathrm{Na}_{2} \mathrm{SO}_{4}$, filtered, and concentrated in vacuo. The reaction mixture was purified by column chromatography (MeOH/DCM) to give 24 as a clear oil (44 mg, 43\%).

1:1 EA/Hex, $\mathrm{R}_{\mathrm{f}}=0.14 .{ }^{1} \mathrm{H}$ NMR $\left(400 \mathrm{MHz}, \mathrm{CDCl}_{3}\right) \delta 4.72(\mathrm{~d}, J=3.5 \mathrm{~Hz}, 1 \mathrm{H}), 3.86(\mathrm{ddd}, J=$ 11.7, 8.9, 5.1 Hz, 1H), 3.59 (dq, $J=9.3,6.2 \mathrm{~Hz}, 2 \mathrm{H}), 3.31(\mathrm{~s}, 3 \mathrm{H}), 3.06$ (t, $J=9.2 \mathrm{~Hz}, 1 \mathrm{H}), 2.11$ $(\mathrm{dd}, J=13.0,5.1 \mathrm{~Hz}, 1 \mathrm{H}), 1.66(\mathrm{ddd}, J=13.1,11.7,3.7 \mathrm{~Hz}, 1 \mathrm{H}), 1.28(\mathrm{~d}, J=6.2 \mathrm{~Hz}, 3 \mathrm{H}) .{ }^{13} \mathrm{C}$ NMR $\left(100 \mathrm{MHz}, \mathrm{CDCl}_{3}\right) \delta$ 98.52, 78.04, 69.19, 67.58, 54.75, 37.80, 17.85.

The NMR spectra are consistent with those reported in the literature. ${ }^{16,17}$ 


\section{C/D) Reductive Amination}

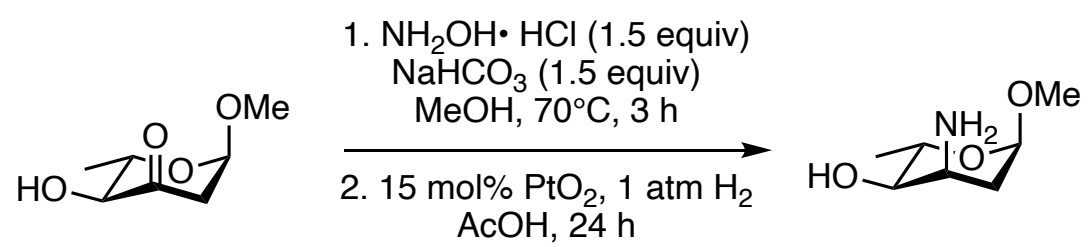

Into a round bottom flask, sugar $2(100 \mathrm{mg}, 0.624 \mathrm{mmol}), \mathrm{NaHCO}_{3}(78.6 \mathrm{mg}, 0.936 \mathrm{mmol})$, $\mathrm{NH}_{2} \mathrm{OH} \cdot \mathrm{HCl}(65 \mathrm{mg}, 0.936 \mathrm{mmol})$ and $6 \mathrm{~mL}$ of anhydrous $\mathrm{MeOH}$ was added with stir bar. The reaction was stirred at $70^{\circ} \mathrm{C}$ for $3 \mathrm{~h}$. After $3 \mathrm{~h}$, the reaction was brought to room temperature, filtered and concentrated under vacuo. (If using older samples of starting material, it is beneficial to run a column (EA/Hex) before the hydrogenation step.) Then, $\mathrm{PtO}_{2}(21 \mathrm{mg}, 0.096 \mathrm{mmol})$ and 6 $\mathrm{mL} \mathrm{AcOH}$ was added. The reaction was sparged with $\mathrm{N}_{2}$ for at least 15 minutes. Then the reaction was sparged with a $\mathrm{H}_{2}$ balloon and let stir under $\mathrm{H}_{2}$ atmosphere for $24 \mathrm{~h}$. After $24 \mathrm{~h}$, the reaction was sparged with $\mathrm{N}_{2}$ for at least 15 minutes to remove all $\mathrm{H}_{2}$. The reaction was then diluted with EtOAc $(6 \mathrm{~mL})$ and filtered through celite that was pre-washed with 1:1 AcOH/EA. The celite was rinsed with EtOAc $(10 \mathrm{~mL})$. The resulting mixture was concentrated under vacuo. To the resulting material was added $10 \mathrm{~mL} 15 \% \mathrm{NaOH}$ and $10 \mathrm{~mL}$ water; the solution was transferred to a separatory funny. The aqueous layer was extracted with EtOAc $(4 \times 10 \mathrm{~mL})$. The organic layer was dried over $\mathrm{Na}_{2} \mathrm{SO}_{4}$, filtered, and concentrated in vacuo to give 25 (85 mg, 84\%).

20:1 DCM/MeOH, $\mathrm{R}_{\mathrm{f}}=0.44 .{ }^{1} \mathrm{H}$ NMR $\left(400 \mathrm{MHz}, \mathrm{CDCl}_{3}\right) \delta 4.66(\mathrm{~d}, J=4.1 \mathrm{~Hz}, 1 \mathrm{H}), 3.62-3.50$ (m, 1H), $3.28(\mathrm{~s}, 3 \mathrm{H}), 3.10(\mathrm{dd}, J=9.7,4.5 \mathrm{~Hz}, 1 \mathrm{H}), 3.01$ (q, $J=3.9 \mathrm{~Hz}, 1 \mathrm{H}), 2.78(\mathrm{~s}, 3 \mathrm{H}), 2.01-$ $1.81(\mathrm{~m}, 2 \mathrm{H}), 1.24(\mathrm{~d}, J=6.2 \mathrm{~Hz}, 3 \mathrm{H}) .{ }^{13} \mathrm{C} \mathrm{NMR}\left(100 \mathrm{MHz}, \mathrm{CDCl}_{3}\right) \delta$ 98.61, 71.05, 64.11, 55.06, 47.71, 36.37, 18.04 .

The NMR spectra are consistent with those reported in the literature. ${ }^{18}$ 


\section{E) Methylation}

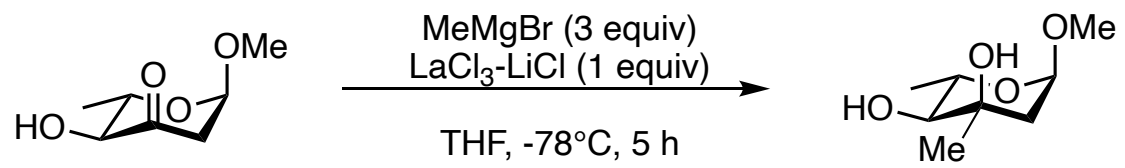

Into a round bottom flask, sugar $2(100 \mathrm{mg}, 0.624 \mathrm{mmol})$ and $12 \mathrm{~mL}$ of anhydrous THF was added with stir bar. The mixture was cooled to $-78{ }^{\circ} \mathrm{C}$. $\mathrm{LaCl}_{3}-\mathrm{LiCl}(1.040 \mathrm{ml}, 0.624 \mathrm{mmol})$ was added dropwise and let stir for $15 \mathrm{~min}$. Then $\mathrm{MeMgBr}(3 \mathrm{M}$ in diethyl ether) (0.624 mL, $1.873 \mathrm{mmol})$ was added dropwise. The resulting mixture was let stir at $-78{ }^{\circ} \mathrm{C}$ for $5 \mathrm{~h}$. The mixture was warmed to room temperature and water $(12 \mathrm{~mL})$ was added to quench. The solution was transferred to a separatory funnel and extracted with ethyl acetate $(5 \times 20 \mathrm{~mL})$. The organic layer was dried over $\mathrm{Na}_{2} \mathrm{SO}_{4}$, filtered, and concentrated in vacuo. The reaction mixture was purified by column chromatography (EtOAc/Hex) to give $\mathbf{2 6}$ as a pale yellow solid (60 mg, 55\%).

1:1 EA/Hex, $\mathrm{R}_{\mathrm{f}}=0.48 .{ }^{1} \mathrm{H}$ NMR $\left(400 \mathrm{MHz}, \mathrm{CDCl}_{3}\right) \delta 4.74(\mathrm{~d}, J=3.6 \mathrm{~Hz}, 1 \mathrm{H}), 3.84(\mathrm{~s}, 1 \mathrm{H}), 3.59$ $(\mathrm{dq}, J=9.6,6.2 \mathrm{~Hz}, 1 \mathrm{H}), 3.36(\mathrm{~s}, 3 \mathrm{H}), 3.01-2.89(\mathrm{~m}, 1 \mathrm{H}), 2.27(\mathrm{~d}, J=11.0 \mathrm{~Hz}, 1 \mathrm{H}), 2.02(\mathrm{dd}, J$ $=14.6,1.2 \mathrm{~Hz}, 1 \mathrm{H}), 1.80(\mathrm{dd}, J=14.6,3.7 \mathrm{~Hz}, 1 \mathrm{H}), 1.32(\mathrm{~d}, J=6.2 \mathrm{~Hz}, 3 \mathrm{H}), 1.22(\mathrm{~s}, 3 \mathrm{H}) .{ }^{13} \mathrm{C}$ NMR (100 MHz, $\left.\mathrm{CDCl}_{3}\right) \delta 98.53,76.52,70.01,65.56,55.12,40.85,25.79,18.06$.

The NMR spectra are consistent with those reported in the literature. ${ }^{19}$ 


\section{F/G) Reductive Amination of Anhydro-sugar Products}

\section{Methyl Oxime Formation:}

Into a round bottom flask, the crude reaction mixture $(5 \times 0.3 \mathrm{mmol}), \mathrm{NaHCO}_{3}(252 \mathrm{mg}, 3.0$ mmol), $\mathrm{NH}_{2} \mathrm{OMe}-\mathrm{HCl}(187.9 \mathrm{mg}, 2.25 \mathrm{mmol})$ and $15 \mathrm{~mL}$ of anhydrous $\mathrm{MeOH}$ was added with stir bar. The reaction was stirred at $70^{\circ} \mathrm{C}$ for $3 \mathrm{~h}$. After $3 \mathrm{~h}$, the reaction was brought to room temperature, filtered, and concentrated under vacuo. The crude reaction was purified by flash column chromatography (EtOAc/DCM) with C2-deactivated silica.

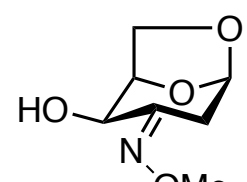

S13: 10 was prepared according to general procedure B using D-anhydrogalactose $(5 \times 0.3 \mathrm{mmol})$. The crude reactions were combined and then subjected to methyl oxime formation (as outlined above). The mixture was purified by column chromatography with C2-deactivated silica (EtOAc/DCM) to give $\mathbf{S 1 3}$ (91 mg, 35\% over two steps).

10:1 DCM/EtOAc, $\mathrm{R}_{\mathrm{f}}=0.69 .[\alpha]^{20} \mathrm{D}=-54.9^{\circ}(\mathrm{c}=1, \mathrm{MeOH}) .{ }^{1} \mathrm{H}$ NMR $(400 \mathrm{MHz}, \mathrm{MeOD}) \delta 5.53$ (s, 1H), 4.53 (t, $J=4.9 \mathrm{~Hz}, 1 \mathrm{H}), 4.46(\mathrm{~d}, J=4.7 \mathrm{~Hz}, 1 \mathrm{H}), 4.06(\mathrm{~d}, J=7.7 \mathrm{~Hz}, 1 \mathrm{H}), 3.88(\mathrm{~s}, 3 \mathrm{H})$, $3.66(\mathrm{dd}, J=7.1,5.7 \mathrm{~Hz}, 1 \mathrm{H}), 3.13(\mathrm{~d}, J=16.3 \mathrm{~Hz}, 1 \mathrm{H}), 2.14(\mathrm{dd}, J=16.4,2.3 \mathrm{~Hz}, 1 \mathrm{H}) .{ }^{13} \mathrm{C}$ NMR (100 MHz, MeOD) $\delta 155.25,101.37,77.15,69.44,65.38,62.08,33.89$. IR (neat, $\left.\mathrm{cm}^{-1}\right): 3458.9$ (br), 2966.9 (br), 2899.8 (w), 1464.8 (w), 1289.6 (w), 1118.2 (s), 1043.6 (s), 943.0 (m). HRMS (DART) $\left([\mathrm{M}+\mathrm{H}]^{+}\right)$Calcd. For C7H12NO4: 174.07608, found 174.07539. 


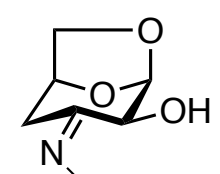

OMe S14: 12 was prepared according to general procedure B using D-anhydromannose (5 $\mathrm{x} 0.3 \mathrm{mmol}$ ). The crude reactions were combined and then subjected to methyl oxime formation (as outlined above). The mixture was purified by column chromatography with C2-deactivated silica (EtOAc/DCM) to give S14 (99 mg, 38\% over two steps).

10:1 DCM/EtOAc, $\mathrm{R}_{\mathrm{f}}=0.47 .[\alpha]^{20} \mathrm{D}=-111.7^{\circ}(\mathrm{c}=1, \mathrm{MeOH}) .{ }^{1} \mathrm{H}$ NMR $(400 \mathrm{MHz}, \mathrm{MeOD}) \delta 5.36$ $(\mathrm{d}, J=2.3 \mathrm{~Hz}, 1 \mathrm{H}), 4.66(\mathrm{t}, J=4.8 \mathrm{~Hz}, 1 \mathrm{H}), 4.14(\mathrm{~d}, J=2.3 \mathrm{~Hz}, 1 \mathrm{H}), 3.87(\mathrm{~s}, 3 \mathrm{H}), 3.76(\mathrm{t}, J=5.3$ $\mathrm{Hz}, 1 \mathrm{H}), 3.65$ (d, $J=7.4 \mathrm{~Hz}, 1 \mathrm{H}), 3.13(\mathrm{~d}, J=16.3 \mathrm{~Hz}, 1 \mathrm{H}), 2.33(\mathrm{dd}, J=16.4,2.8 \mathrm{~Hz}, 1 \mathrm{H}) .{ }^{13} \mathrm{C}$ NMR (100 MHz, MeOD) $\delta$ 155.47, 103.67, 73.59, 71.61, 70.46, 62.09, 31.61. IR (neat, cm-1): 3470.1 (br), 2963.2 (br), 2899.8 (w), 1729.5 (w), 1640.0 (w), 1230.0 (w), 1088.4 (s), 1043.6 (s), $965.4(\mathrm{~m})$. HRMS (DART) $\left([\mathrm{M}+\mathrm{H}]^{+}\right)$Calcd. For C7H12NO4: 174.07608, found 174.07585.

\section{Raney Nickel hydrogenation:}

Into a round bottom flask, Raney Nickel (535 mg, 5 equiv by wt.) was added as a slurry in water with a stir bar under $\mathrm{N}_{2}$. The Raney Nickel was washed with water $(10 \mathrm{ml})$ once and then with $\mathrm{MeOH}(10 \mathrm{ml}) .4 \mathrm{~mL}$ of $\mathrm{NH}_{3}(7 \mathrm{M}$ in $\mathrm{MeOH})$ was added. In a separate round bottom flask, methyl oxime starting material (107 mg, $0.62 \mathrm{mmol}$ ) was dissolved in $4 \mathrm{~mL}$ of anhydrous $\mathrm{MeOH}$. Then, the oxime starting material in $\mathrm{MeOH}$ was added to Raney Nickel solution. $\mathrm{A} \mathrm{H}_{2}$ balloon was added to create a $\mathrm{H}_{2}$ atmosphere. The reaction was stirred for $5 \mathrm{~h}$ at rt. After $5 \mathrm{~h}$, the $\mathrm{H}_{2}$ balloon was removed and the round bottom was placed under $\mathrm{N}_{2}$ and the head space was evacuated with $\mathrm{N}_{2}$. The reaction was then filtered through celite, washed with $\mathrm{MeOH}(15 \mathrm{~mL})$, and concentrated under vacuo. The crude reaction was purified by column chromatography with C2-deactived silica $(\mathrm{MeOH} / \mathrm{DCM})$. 


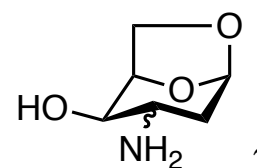

27: S13 was subjected to hydrogenation (as outlined above). The mixture was purified by column chromatography with C2-deactivated silica (MeOH/DCM) to give 27 as a 10:1 mixture (58 mg, 64\%).

Major isomer ( $\mathrm{NH}_{2}$-axial): ${ }^{1} \mathrm{H}$ NMR $(400 \mathrm{MHz}, \mathrm{MeOD}) \delta 5.52(\mathrm{~s}, 1 \mathrm{H}), 4.35(\mathrm{t}, \mathrm{J}=4.5 \mathrm{~Hz}, 1 \mathrm{H})$, $4.27(\mathrm{~d}, \mathrm{~J}=8.1 \mathrm{~Hz}, 1 \mathrm{H}), 4.01(\mathrm{t}, \mathrm{J}=5.4 \mathrm{~Hz}, 1 \mathrm{H}), 3.61(\mathrm{dd}, \mathrm{J}=7.9,4.7 \mathrm{~Hz}, 1 \mathrm{H}), 3.38(\mathrm{t}, \mathrm{J}=6.2 \mathrm{~Hz}$, 1H), $2.09-1.93(\mathrm{~m}, 2 \mathrm{H}) .{ }^{13} \mathrm{C}$ NMR (100 MHz, MeOD) $\delta$ 101.62, 76.37, 65.63, 65.31, 48.62, 36.02 .

Minor isomer ( $\mathrm{NH}_{2}$-equatorial): ${ }^{1} \mathrm{H} \mathrm{NMR}\left(400 \mathrm{MHz}, \mathrm{CD}_{3} \mathrm{OD}\right) \delta 5.45$ (s, $\left.1 \mathrm{H}\right), 4.34-4.39$ (m, 1H), $4.05(\mathrm{~d}, J=7.4 \mathrm{~Hz}, 1 \mathrm{H}), 3.63-3.56(\mathrm{~m}, 1 \mathrm{H}), 3.48(\mathrm{dd}, J=9.0,4.0 \mathrm{~Hz}, 1 \mathrm{H}), 2.93-2.86(\mathrm{~m}, 1 \mathrm{H})$, $2.05-1.90(\mathrm{~m}, 1 \mathrm{H}), 1.45(\mathrm{t}, J=12.2 \mathrm{~Hz}, 1 \mathrm{H}) .{ }^{13} \mathrm{C} \mathrm{NMR}\left(100 \mathrm{MHz}, \mathrm{CD}_{3} \mathrm{OD}\right) \delta 100.7,75.6,73.2$, $64.3,48.6,39.0$.

$\mathrm{MeOH}, \mathrm{R}_{\mathrm{f}}=0.06 . \mathrm{IR}\left(\right.$ neat, $\left.\mathrm{cm}^{-1}\right): 3365.8(\mathrm{br}), 2959.5(\mathrm{~m}), 2899.8(\mathrm{~m}), 1587.8(\mathrm{w}), 1125.6(\mathrm{~s})$, 864.7 (s). HRMS (DART) $\left([\mathrm{M}+\mathrm{H}]^{+}\right)$Calcd. For C6H12NO3: 146.08117, found 146.08167. 


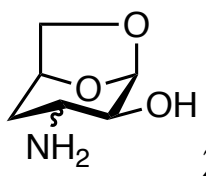

28: S14 was subjected to hydrogenation (as outlined above). The mixture was purified by column chromatography with $\mathrm{C} 2$-deactivated silica $(\mathrm{MeOH} / \mathrm{DCM})$ to give $\mathbf{2 8}$ as a 2:1 mixture (55 mg, 61\%).

Major isomer ( $\mathrm{NH}_{2}$-axial): ${ }^{1} \mathrm{H}$ NMR (400 MHz, MeOD) $\delta 5.26(\mathrm{~s}, 1 \mathrm{H}), 4.56-4.45(\mathrm{~m}, 1 \mathrm{H}), 4.04$ $(\mathrm{d}, J=7.6 \mathrm{~Hz}, 1 \mathrm{H}), 3.67(\mathrm{ddd}, J=7.3,4.9,1.5 \mathrm{~Hz}, 1 \mathrm{H}), 3.60(\mathrm{dd}, J=5.9,1.6 \mathrm{~Hz}, 1 \mathrm{H}), 3.21(\mathrm{t}, J$ $=6.0 \mathrm{~Hz}, 1 \mathrm{H}), 2.23(\mathrm{dddd}, J=15.1,6.2,4.2,1.7 \mathrm{~Hz}, 1 \mathrm{H}), 1.94-1.85(\mathrm{~m}, 1 \mathrm{H}) .{ }^{13} \mathrm{C}$ NMR $(151$ $\left.\mathrm{MHz}, \mathrm{D}_{2} \mathrm{O}\right) \delta 102.10,72.73,68.08,67.97,47.03,33.30$.

Minor isomer ( $\mathrm{NH}_{2}$-equatorial): ${ }^{1} \mathrm{H}$ NMR (400 MHz, $\left.\mathrm{CD}_{3} \mathrm{OD}\right) \delta 5.22(\mathrm{~s}, 1 \mathrm{H}), 4.56(\mathrm{~m}, 1 \mathrm{H}), 3.84$ $(\mathrm{d}, J=7.2 \mathrm{~Hz}, 1 \mathrm{H}), 3.74-3.68(\mathrm{~m}, 1 \mathrm{H}), 3.20(\mathrm{~d}, J=8.7 \mathrm{~Hz}, 1 \mathrm{H}), 2.95-2.9(\mathrm{~m}, 1 \mathrm{H}), 1.98-1.91$ (m, 1H), 1.63 (t, $J=14.1 \mathrm{~Hz}, 1 \mathrm{H}) .{ }^{13} \mathrm{C} \mathrm{NMR}\left(100 \mathrm{MHz}, \mathrm{CD}_{3} \mathrm{OD}\right) \delta 102.2,76.4,73.3,67.9,48.8$, 35.8 .

$\mathrm{MeOH}, \mathrm{R}_{\mathrm{f}}=$ 0.05. IR (neat, $\mathrm{cm}^{-1}$ ): $3354.6(\mathrm{br}), 2899.8(\mathrm{~m}), 1599.0(\mathrm{~m}), 1341.8(\mathrm{w}), 1080.9(\mathrm{~s})$, 976.5 (m). HRMS (DART) $\left([\mathrm{M}+\mathrm{H}]^{+}\right)$Calcd. For C6H12NO3: 146.08117, found 146.08082. 


\section{Condition Optimization}

A) Variation of standard reagents in the redox isomerization of $\alpha$-methylrhamnoside 1

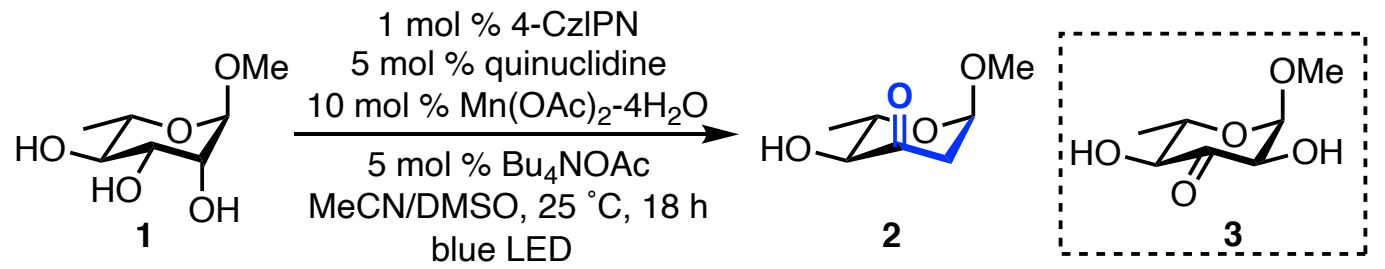

Table S1. Reaction optimization.

\begin{tabular}{|c|c|c|c|c|}
\hline Entry & Variation from standard conditions & Yield $2(\%)$ & $\operatorname{RSM}(\%)$ & Notes \\
\hline 1 & none & 50 & 5 & \\
\hline \multicolumn{5}{|c|}{ Bases (instead of $5 \mathrm{~mol} \mathrm{\%} \mathrm{NBu}_{4} \mathrm{OAc}$ ) } \\
\hline 2 & $10 \mathrm{~mol} \% \mathrm{KOAc}$ & 37 & $<5$ & \\
\hline 3 & $10 \mathrm{~mol} \% \mathrm{KOBz}$ & 38 & $<5$ & \\
\hline 4 & $5 \mathrm{~mol} \% \mathrm{NBu}_{4} \mathrm{OBz}$ & 43 & $<5$ & \\
\hline 5 & $5 \mathrm{~mol} \% \mathrm{NBu}_{4} \mathrm{OBz}(4-\mathrm{Cl})$ & 44 & $<5$ & \\
\hline \multicolumn{5}{|c|}{ Manganese Source (instead of $10 \mathrm{~mol} \% \mathrm{Mn}(\mathrm{OAc})_{2}-4 \mathrm{H}_{2} \mathrm{O}$ ) } \\
\hline 6 & $\mathrm{Mn}(\mathrm{OAc})_{2}-4 \mathrm{H}_{2} \mathrm{O}, 99+\%$ & 48 & 7 & \\
\hline 7 & $\mathrm{Mn}(\mathrm{OAc})_{3}-2 \mathrm{H}_{2} \mathrm{O}$ & 44 & 4 & \\
\hline 8 & $\mathrm{MnCl}_{2}$ & $<5$ & 90 & \\
\hline 9 & $\operatorname{Mn}(\mathrm{acac})_{3}$ & $<5$ & 82 & \\
\hline \multicolumn{5}{|c|}{ Photocatalyst (instead of $2 \mathrm{~mol} \%$ 4-CzIPN) } \\
\hline 10 & $1 \mathrm{~mol} \%\left(\operatorname{IrdF}\left(\mathrm{CF}_{3}\right) \mathrm{ppy}_{2}(\mathrm{dtbpy})\right) \mathrm{PF}_{6}$ & 40 & 15 & \\
\hline 11 & $1 \mathrm{~mol} \% \operatorname{Ir}(\mathrm{dF}(\mathrm{Me}) \mathrm{ppy})_{2}(\mathrm{dtbbpy}) \mathrm{PF}_{6}$ & 36 & 20 & \\
\hline \multicolumn{5}{|c|}{ Negative Controls } \\
\hline 12 & No $\mathrm{Mn}(\mathrm{OAc})_{2}-4 \mathrm{H}_{2} \mathrm{O}$ & 2 & 85 & $9 \% 3$ \\
\hline 13 & No quinuclidine & 0 & 100 & \\
\hline 14 & No 4-CzIPN & 0 & 100 & \\
\hline 15 & No light & 0 & 95 & \\
\hline
\end{tabular}


B) Examining Lewis bases and Brønsted acids to promote the redox isomerization of $\alpha$ methylrhamnoside 1

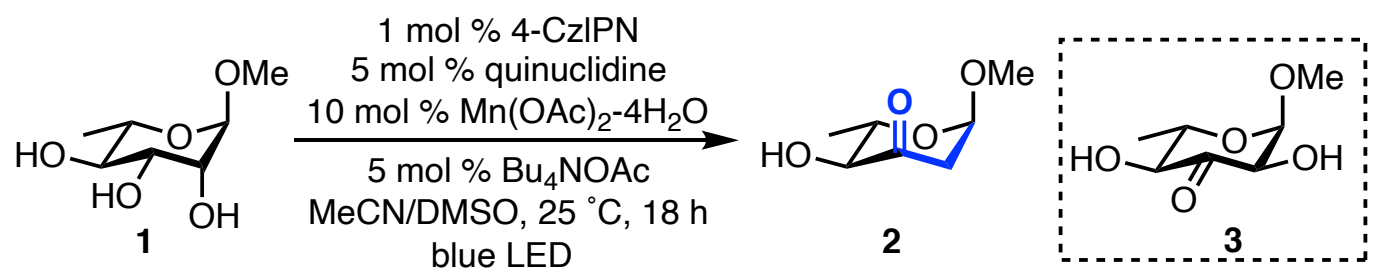

Table S2. Lewis bases and Brønsted acids in place of [Mn] do not promote formation of 2.

\begin{tabular}{|c|c|c|c|c|}
\hline Entry & Variation from standard conditions & Yield $2(\%)$ & $\operatorname{RSM}(\%)$ & Notes \\
\hline 1 & none & 50 & 5 & \\
\hline \multicolumn{5}{|c|}{ Lewis Bases (instead of $5 \mathrm{~mol} \% \mathrm{NBu}_{4} \mathrm{OAc}$ and $10 \mathrm{~mol} \% \mathrm{Mn}(\mathrm{OAc})_{2}-4 \mathrm{H}_{2} \mathrm{O}$ ) } \\
\hline 2 & $5 \mathrm{~mol} \% \mathrm{NBu}_{4} \mathrm{OAc}$ & 2 & 85 & $9 \% 3$ \\
\hline 3 & $5 \mathrm{~mol} \% \mathrm{NBu}_{4} \mathrm{OBz}$ & 3 & 70 & $7 \% 3$ \\
\hline 4 & $5 \mathrm{~mol} \% \mathrm{NBu}_{4} \mathrm{OBz}(4-\mathrm{Cl})$ & 3 & 71 & $6 \% 3$ \\
\hline 5 & $5 \mathrm{~mol} \% \mathrm{NBu}_{4} \mathrm{OP}(\mathrm{O})(\mathrm{OBu})_{2}$ & 3 & 90 & $6 \% 3$ \\
\hline \multicolumn{5}{|c|}{ Brønsted Acids (instead of $5 \mathrm{~mol} \% \mathrm{NBu}_{4} \mathrm{OAc}$ and $10 \mathrm{~mol} \% \mathrm{Mn}(\mathrm{OAc})_{2}-4 \mathrm{H}_{2} \mathrm{O}$ ) } \\
\hline 6 & $20 \mathrm{~mol} \mathrm{\%} \mathrm{HOBz}$ & 1 & 92 & \\
\hline 7 & $20 \mathrm{~mol} \% \mathrm{HOAc}$ & 3 & 88 & \\
\hline 8 & $20 \mathrm{~mol} \% \mathrm{pTsOH}$ & 0 & 99 & \\
\hline 9 & $20 \mathrm{~mol}_{\%} \mathrm{NH}_{4} \mathrm{Cl}$ & 0 & 86 & \\
\hline
\end{tabular}


C) Examining Lewis acids to promote redox isomerization

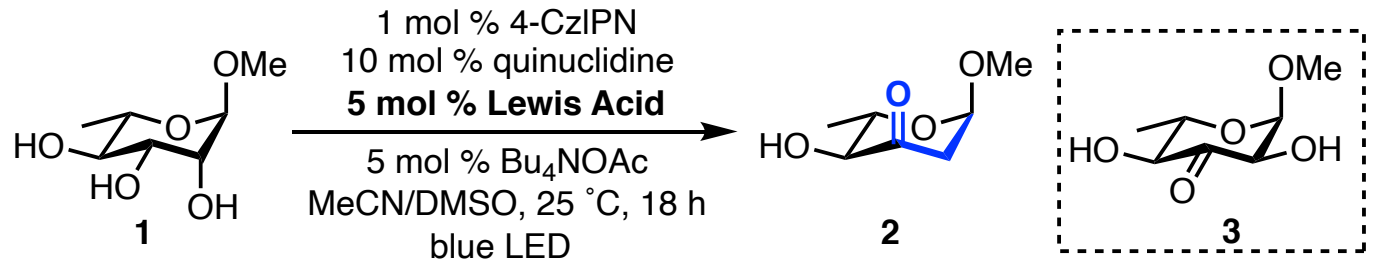

Table S3. Lewis acids in place of [Mn] do not promote formation of 2.

\begin{tabular}{|c|c|c|c|c|}
\hline Entry & Lewis Acid & Yield 2 (\%) & RSM (\%) & Notes \\
\hline 1 & $\mathrm{PhB}(\mathrm{OH})_{2}$ & 3 & 85 & $8 \% 3$ \\
\hline 2 & $\mathrm{C}_{6} \mathrm{~F}_{5} \mathrm{~B}(\mathrm{OH})_{2}$ & 1 & 78 & $7 \% 3$ \\
\hline 3 & $\mathrm{Mes}_{2} \mathrm{BOH}$ & 1 & 90 & $3 \% 3$ \\
\hline 4 & $\mathrm{Cu}(\mathrm{OAc})_{2}$ & 5 & $<5$ & $38 \% 3$ \\
\hline 5 & $\mathrm{NiCl}_{2}$-glyme & 1 & 81 & $6 \% 3$ \\
\hline 6 & $\mathrm{Co}(\mathrm{OAc})_{2}-4 \mathrm{H}_{2} \mathrm{O}$ & 1 & 38 & $18 \% 3$ \\
\hline 7 & $\mathrm{Zn}(\mathrm{OAc})_{2}-2 \mathrm{H}_{2} \mathrm{O}$ & 5 & 66 & $4 \% 3$ \\
\hline 8 & $\mathrm{Zn}(\mathrm{OTf})_{2}$ & 5 & 58 & $10 \% 3$ \\
\hline 9 & $\mathrm{Zn}\left(\mathrm{BF}_{4}\right)_{2}-\mathrm{H}_{2} \mathrm{O}$ & 2 & 84 & $2 \% 3$ \\
\hline 10 & $\mathrm{Al}(\mathrm{OiPr})_{3}$ & 5 & 74 & $4 \% 3$ \\
\hline 11 & $\mathrm{AlCl}_{3}$ & 1 & 93 & $1 \% 3$ \\
\hline 12 & $\mathrm{La}(\mathrm{OAc})_{3}$ & 5 & 76 & $4 \% 3$ \\
\hline 13 & $\operatorname{Er}(\mathrm{OTf})_{3}$ & 4 & 60 & $3 \% 3$ \\
\hline 14 & $\mathrm{Yb}(\mathrm{OTf}) 3$ & 3 & 82 & $4 \% 3$ \\
\hline 15 & $\mathrm{~Tb}(\mathrm{OTf})_{3}$ & 2 & 66 & $3 \% 3$ \\
\hline 16 & $\mathrm{Sc}(\mathrm{OTf})$ & 5 & 76 & $4 \% 3$ \\
\hline
\end{tabular}



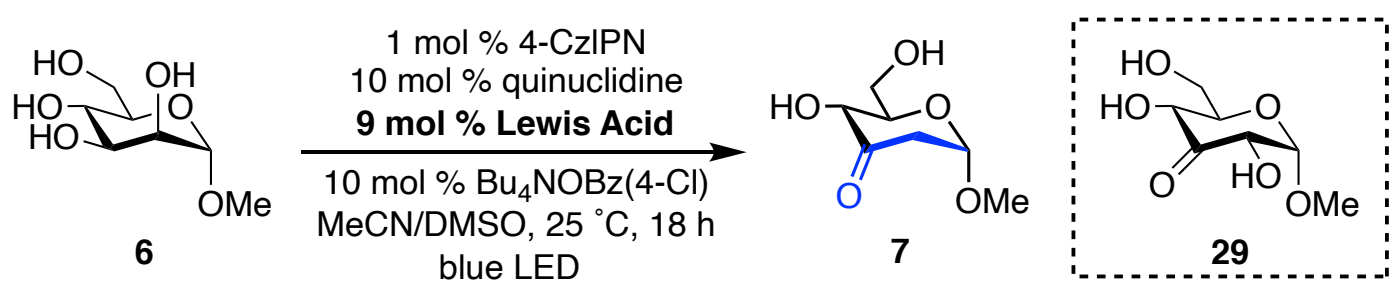

Table S4. Lewis acids in place of [Mn] do not promote formation of 7.

\begin{tabular}{|c|c|c|c|c|}
\hline Entry & Variation from standard conditions & Yield 7 (\%) & $\operatorname{RSM}(\%)$ & Notes \\
\hline 1 & $\mathrm{FeCl}_{3}-6 \mathrm{H}_{2} \mathrm{O}$ & - & 66 & \\
\hline 2 & $\mathrm{FeCl}_{3}$ & - & 62 & \\
\hline 3 & $\mathrm{Fe}\left(\mathrm{NO}_{3}\right)_{3}-9 \mathrm{H}_{2} \mathrm{O}$ & - & 72 & \\
\hline 4 & $\mathrm{Fe}(\mathrm{acac})_{3}$ & - & 55 & \\
\hline 5 & $\mathrm{FeCl}_{2}-4 \mathrm{H}_{2} \mathrm{O}$ & - & 57 & \\
\hline 6 & $\mathrm{Fe}(\mathrm{OAc})_{2}$ & - & 76 & \\
\hline 7 & $\left(\mathrm{NH}_{4}\right)_{2} \mathrm{Ce}\left(\mathrm{NO}_{3}\right)_{6}(\mathrm{CAN})$ & - & 70 & \\
\hline 8 & $\mathrm{Ce}(\mathrm{OAc})_{3}-\mathrm{xH}_{2} \mathrm{O}$ & 4 & 70 & \\
\hline 9 & $\mathrm{Ce}\left(\mathrm{SO}_{4}\right)_{2}-\mathrm{xH}_{2} \mathrm{O}$ & 4 & 81 & \\
\hline 10 & $\mathrm{Ti}(\mathrm{OiPr})_{3}$ & 4 & 74 & $10 \% 29$ \\
\hline 11 & $\operatorname{Ti}\left(\mathrm{cp}^{*}\right)_{2}$ & 1 & 82 & $3 \% 29$ \\
\hline 12 & $\mathrm{SmI}_{2}$ & 2 & 90 & \\
\hline 13 & $\mathrm{C}_{6} \mathrm{~F}_{5} \mathrm{~B}(\mathrm{OH})_{2}$ & 1 & - & $5 \% 29$ \\
\hline 14 & 2-OMe- $\mathrm{PhB}(\mathrm{OH})_{2}$ & 2 & 68 & $3 \% 29$ \\
\hline
\end{tabular}


D) Examining the effect of water on the redox isomerization of $\alpha$-methylrhamnoside 1

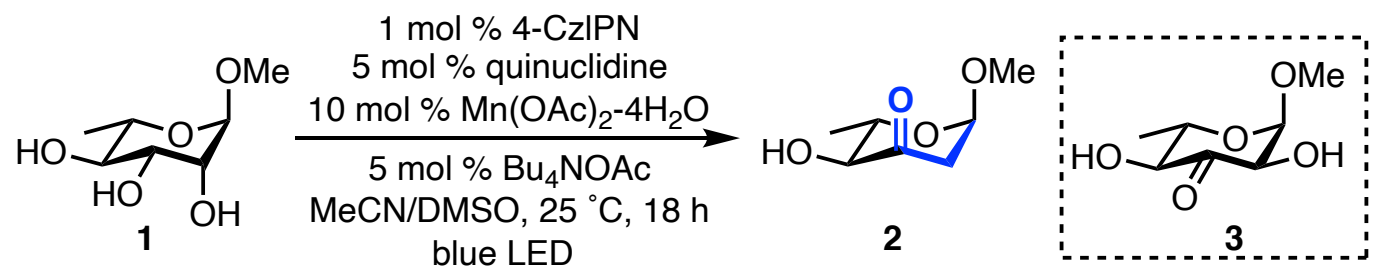

Table S5. Water does not influence the formation of 2.

\begin{tabular}{|c|c|c|c|c|}
\hline Entry & Variation from standard conditions & Yield $2(\%)$ & RSM (\%) & Notes \\
\hline 1 & none & 50 & 5 & \\
\hline \multicolumn{5}{|c|}{ Manganese Source (instead of $10 \mathrm{~mol} \% \mathrm{Mn}(\mathrm{OAc})_{2}-4 \mathrm{H}_{2} \mathrm{O}$ ) } \\
\hline 2 & $\mathrm{Mn}(\mathrm{OAc})_{2}$ (anhydrous) & 50 & 10 & \\
\hline 3 & $\mathrm{Mn}\left(\mathrm{OOC}\left(\mathrm{CH}_{2}\right)_{3} \mathrm{C}_{6} \mathrm{H}_{11}\right)_{2}$ & 47 & 8 & \\
\hline \multicolumn{5}{|c|}{ Molecular Sieves } \\
\hline 4 & $20 \mathrm{mg} 4 \AA \mathrm{MS}$ & 43 & - & \\
\hline 5 & $60 \mathrm{mg} 4 \AA \mathrm{MS}$ & 48 & - & \\
\hline 6 & $20 \mathrm{mg} 5 \AA \mathrm{MS}$ & 44 & - & \\
\hline 7 & $60 \mathrm{mg} 5 \AA \mathrm{MS}$ & 45 & - & \\
\hline \multicolumn{5}{|c|}{ Addition of $\mathrm{H}_{2} \mathrm{O}$} \\
\hline 8 & 0.1 equiv $\mathrm{H}_{2} \mathrm{O}$ & 46 & - & \\
\hline 9 & 0.5 equiv $\mathrm{H}_{2} \mathrm{O}$ & 49 & - & \\
\hline 10 & 1.0 equiv $\mathrm{H}_{2} \mathrm{O}$ & 48 & 2 & \\
\hline 11 & 5.0 equiv $\mathrm{H}_{2} \mathrm{O}$ & 44 & 7 & \\
\hline 12 & 10.0 equiv $\mathrm{H}_{2} \mathrm{O}$ & 32 & 29 & \\
\hline
\end{tabular}

MS activated in a vacuum oven at $150{ }^{\circ} \mathrm{C}$ for 3 days 


\section{Mechanistic Studies}

\section{A) Hydrogen Atom Abstraction Site-Selectivity}

a) Subjection of anhydro sugars to SCS and epimerization reaction condition. ${ }^{20,21}$

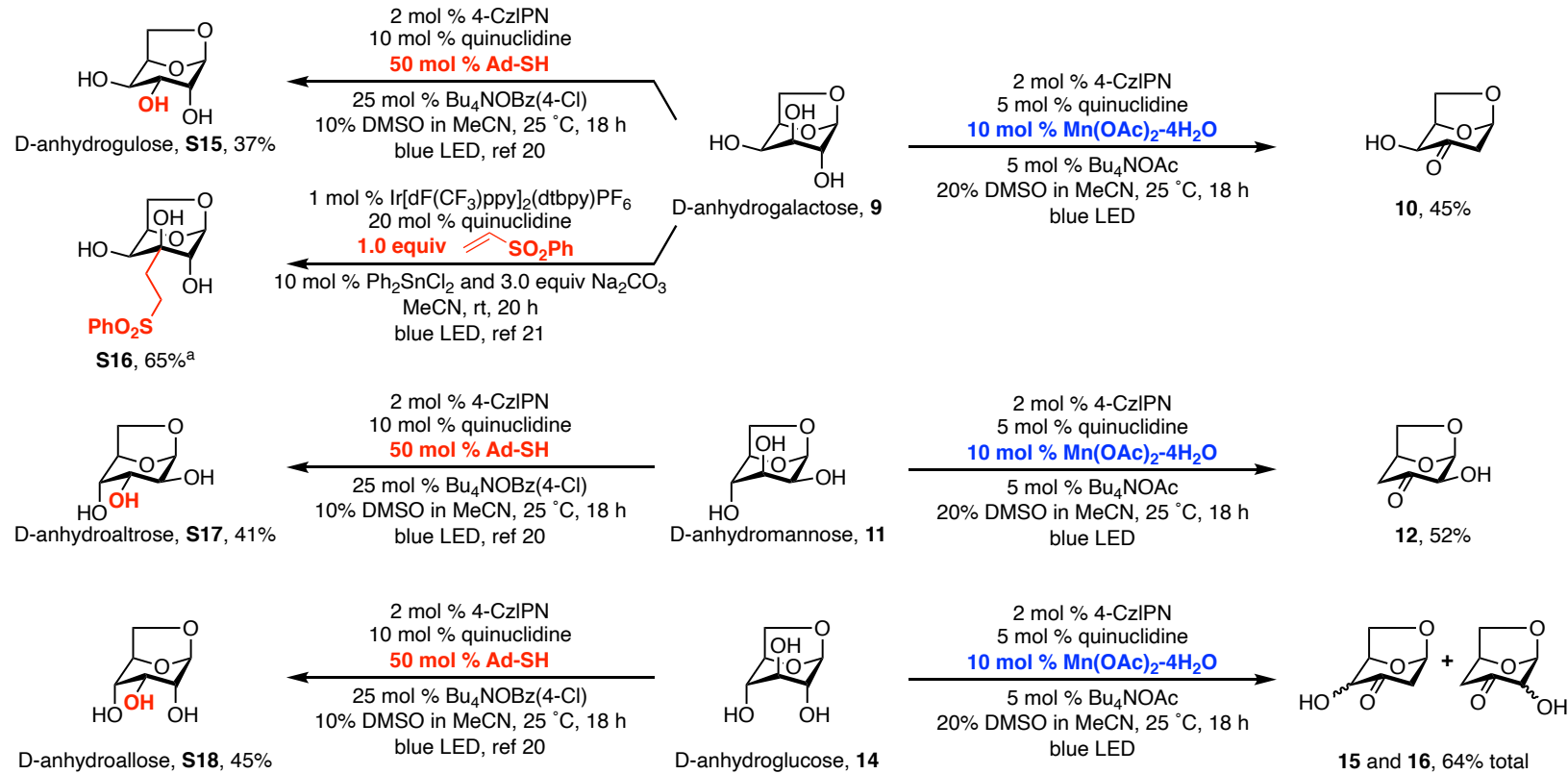

${ }^{\text {a}}$ Reaction performed with 2 equivalents of 9

\section{b) Subjection of $\boldsymbol{\alpha}$-methylglucoside to SCS and epimerization reaction conditions. ${ }^{20,22}$}

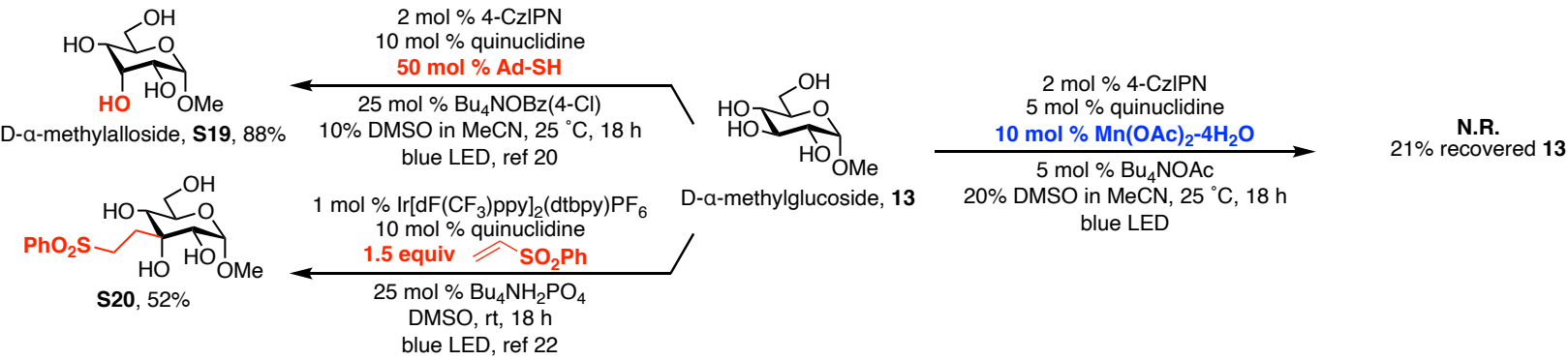

\section{c) Subjection of $\alpha$-methylgalactoside to SCS and epimerization reaction conditions. ${ }^{20}$}

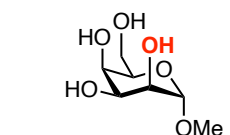

D-a-methyltaloside, $\mathbf{S 2 1}, 15 \%$

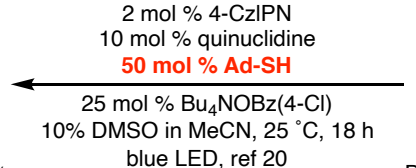

blue LED, ref 20

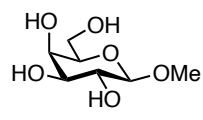

D-a-methylgalactoside, 19

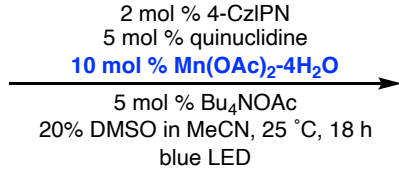

$25 \%$ recovered 19 


\section{B) Mechanistic Reactions}

a) Crossover study with anhydrogalactose and 2

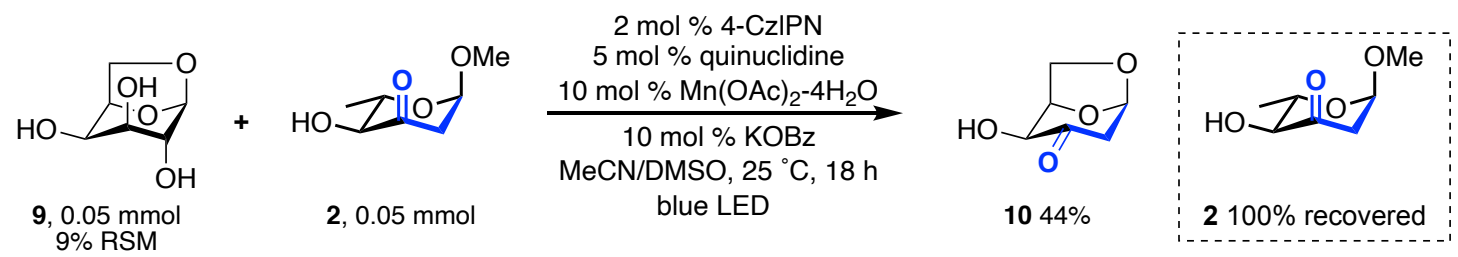

b) Crossover study with anhydromannose and 7
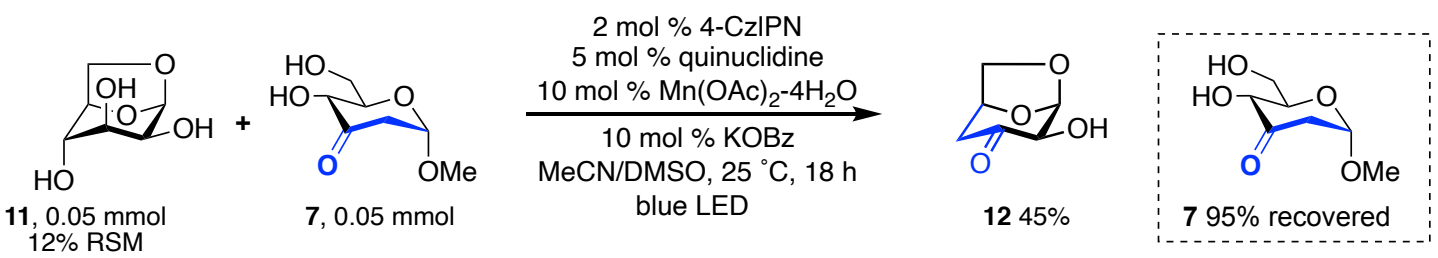

c) Crossover study with anhydrogalactose and 3-keto-glucoside, 29<smiles>OC1C2COC(O2)C1O</smiles>

9, $0.09 \mathrm{mmol}$ $9 \%$ RSM

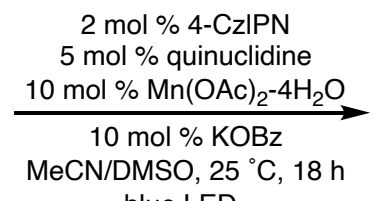

blue LED

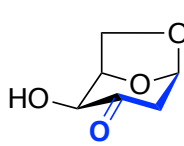

$1041 \%$

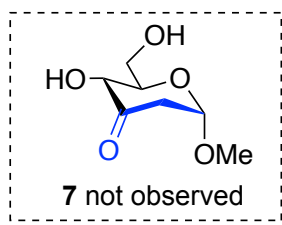

d) Low-conversion crossover study with anhydrogalactose and 3-keto-glucoside, 29<smiles>OC1COC2COC1C2O</smiles>

9, $0.05 \mathrm{mmol}$ $69 \%$ RSM

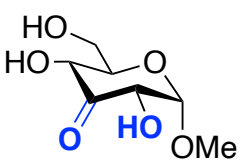

$29,0.05 \mathrm{mmol}$ $26 \%$ RSM

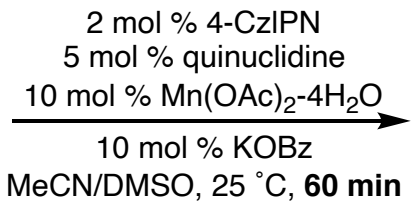

blue LED

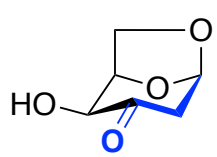

$1011 \%$

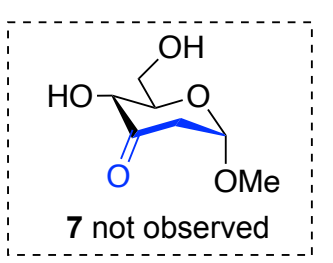

e) Crossover study with anhydrogalactose and 3-keto-rhamnoside, 30

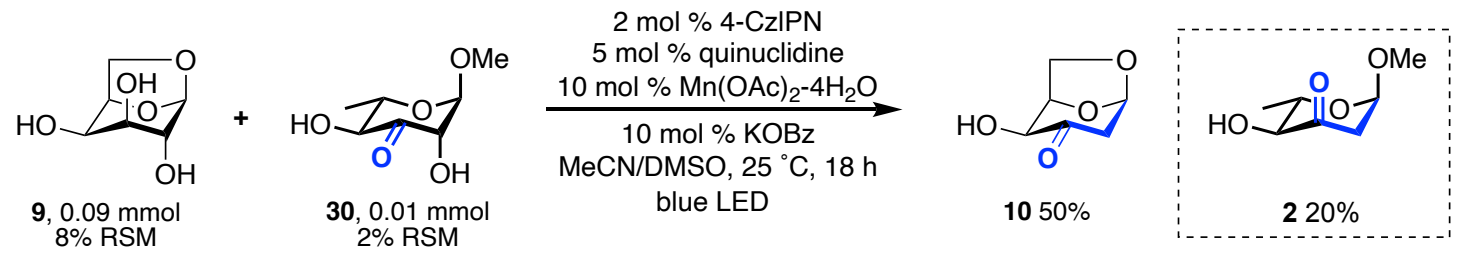

f) Subjection of 3-keto-rhamnoside, 30, to standard reaction conditions

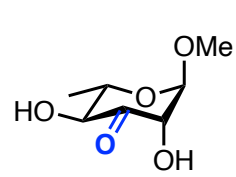

$30,0.1 \mathrm{mmol}$ $18 \%$ RSM

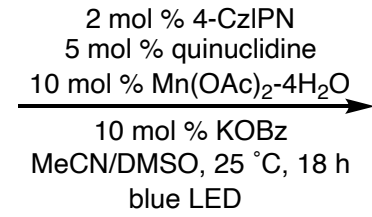

$\underbrace{\mathrm{OMe}}_{314 \%}$

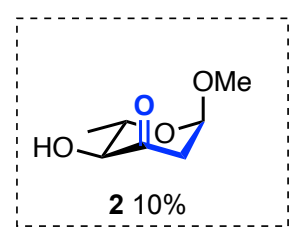




\section{C) Investigation of Mass Balance}

\section{a) Crude ${ }^{1} \mathbf{H}$ NMR of $\alpha$-methylrhamnoside 1 subjected to standard reaction conditions}

Despite nearly full consumption of starting material, no other significant $(>3 \%)$ byproduct is detected in the crude reaction mixtures.
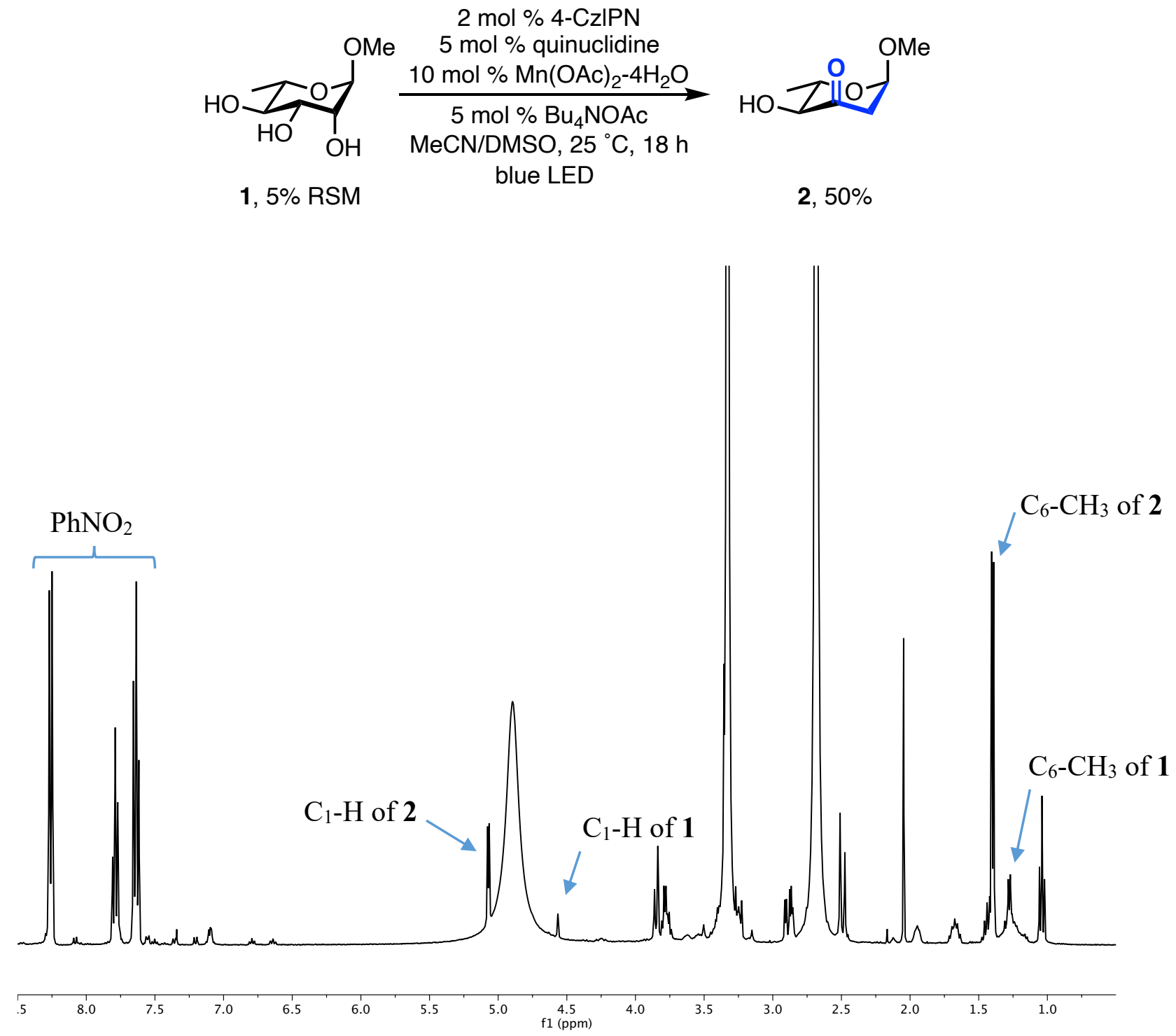

Figure S2. Crude ${ }^{1} \mathrm{H}$ NMR of $\alpha$-methylrhamnoside 1 subjected to standard reaction conditions, as described in general procedure A. Reaction was quantitated using nitrobenzene as an external standard; same reaction as seen in Table S1, entry 1. 


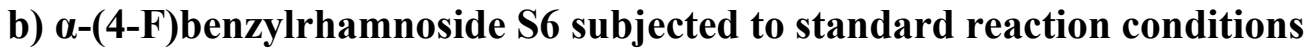

To more closely monitor the formation of potentially volatile reaction side-products, we carried out an experiment using 4-fluoromethylbenzyl rhamnoside S6. Analysis of the crude reaction mixture by ${ }^{1} \mathrm{H}$ and ${ }^{19} \mathrm{~F}$ NMR revealed the formation of $43 \%$ product $\mathbf{S 1 2}$ along with $19 \%$ unreacted S6, and 31\% 4-fluoromethylbenzyl alcohol (Figure S3), reflecting perfect mass balance with respect to the aglycone, but only $64 \%$ with respect to the glycoside.

Analytical standards for the 3-keto sugars, S22 and S23 were synthesized from S6 using Waymouth's selective catalytic oxidation of carbohydrates using $[(\text { neocuproine }) \mathrm{Pd}(\mathrm{OAc})]_{2}(\mathrm{OTf})_{2}{ }^{23} \mathbf{S 2 2}$ was made using the preparative scale trifluoroethanol conditions, while $\mathbf{S 2 3}$ was made using the preparative scale $\mathrm{MeCN} / \mathrm{H}_{2} \mathrm{O}$ conditions. 

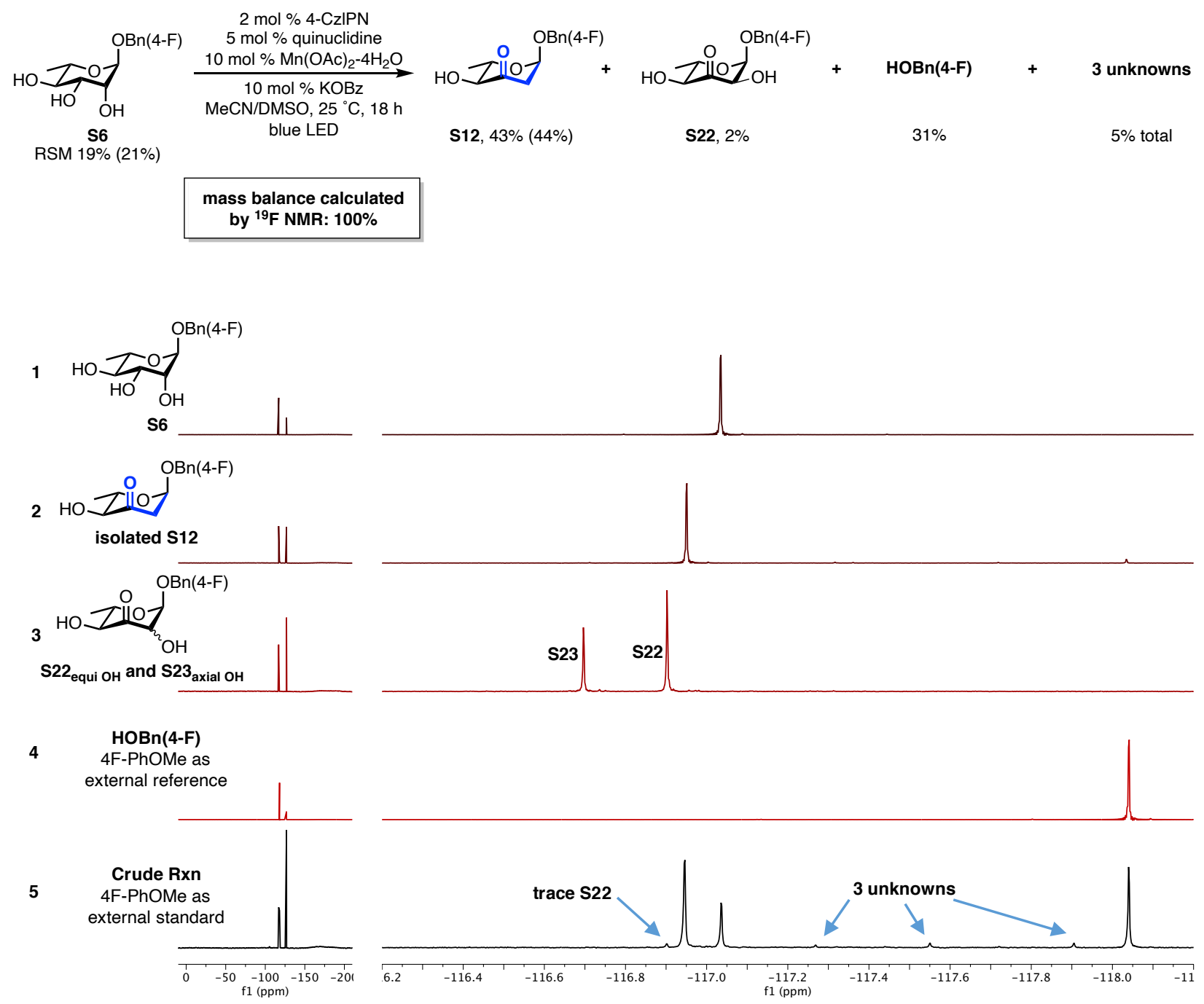

Figure S3. ${ }^{19} \mathrm{~F}$ NMR stack of relevant analytical standards and the crude NMR of S6 subjected to standard reaction conditions (as described in general procedure $\mathrm{A}$, except 4F-PhOMe was used as the external standard instead of $\mathrm{PhNO}_{2}$ ). 4F-PhOMe was added to every NMR as an external reference to align the spectra. The left stack shows the whole domain of the ${ }^{19} \mathrm{~F}$ NMR with $4 \mathrm{~F}$ PhOMe appearing at $-126.8 \mathrm{ppm}$ and all other peaks within the range of -116.2 to $-118.2 \mathrm{ppm}$. The right stack shows the domain of the ${ }^{19} \mathrm{~F}$ NMR between -116.2 to $-118.2 \mathrm{ppm}$. 


\section{c) Crude ${ }^{13} \mathrm{C}$ NMRs of ${ }^{13} \mathrm{C}$ labeled mannosides subjected to modified reaction conditions}

To more closely monitor the formation side-products originating from the degradation of the glycoside, we carried out a series of experiments with ${ }^{13} \mathrm{C}$ labeled mannosides. Subjection of a mannoside with a ${ }^{13} \mathrm{C}$ labeled aglycone, S9, resulted in the formation of product, labeled 7 , and methanol (Figure S4 and S5, entry 5). Other than remaining starting material, no other peaks from possible side products are visible. Subjection of mannosides with a ${ }^{13} \mathrm{C}$ labeled $\mathrm{C}_{1}(\mathbf{S 1 0})$ or $\mathrm{C}_{1-6}$, (S11) resulted in the formation of product, labeled 7, and ${ }^{13} \mathrm{CO}_{2}$ (Figure S4 and S5, entries 6 and 7). Other than remaining starting material, only very minor peaks of side-products are visible. 


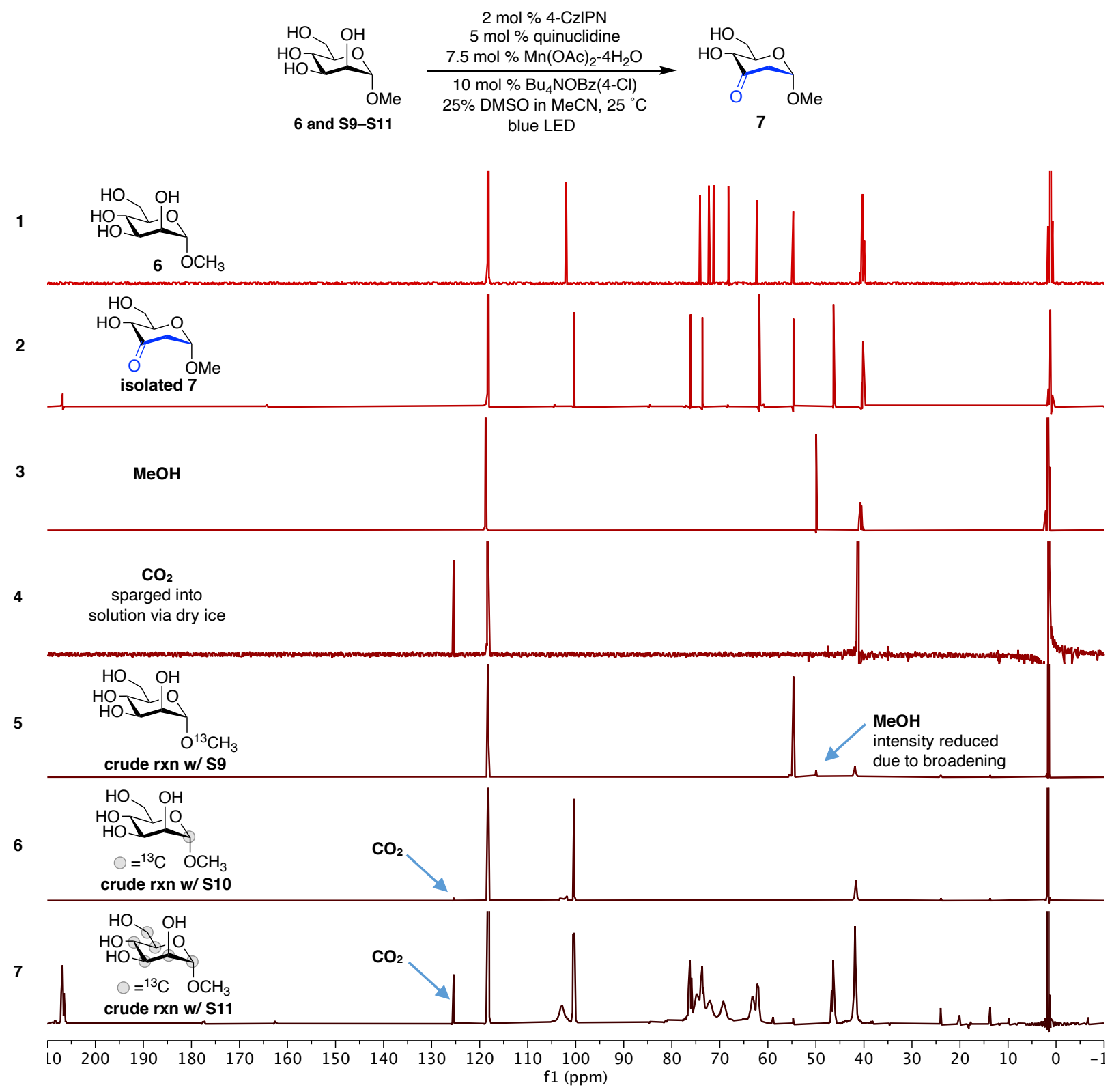

Figure S4. ${ }^{13} \mathrm{C}$ NMR stack of relevant analytical standards and the crude NMRs of 6 and S9-S11 subjected to modified reaction conditions from those described in general procedure A, see scheme above. The reactions were performed at $0.1 \mathrm{M}$ (instead of $0.2 \mathrm{M}$, to reduce broadening) in a sealed J-young tube. $0.05 \mathrm{mmol}$ of the corresponding mannoside was added to the J-young tube directly. All other reagents were prepared at the corresponding concentration in a stock solution and added to the J-young tubes via syringe in the purge box. The reactions were irradiated with blue LED for $18 \mathrm{~h}$ with no stirring. Upon reaction completion, ${ }^{13} \mathrm{C}$ NMRs were acquired on the Bruker 500 with 1024 scans. 

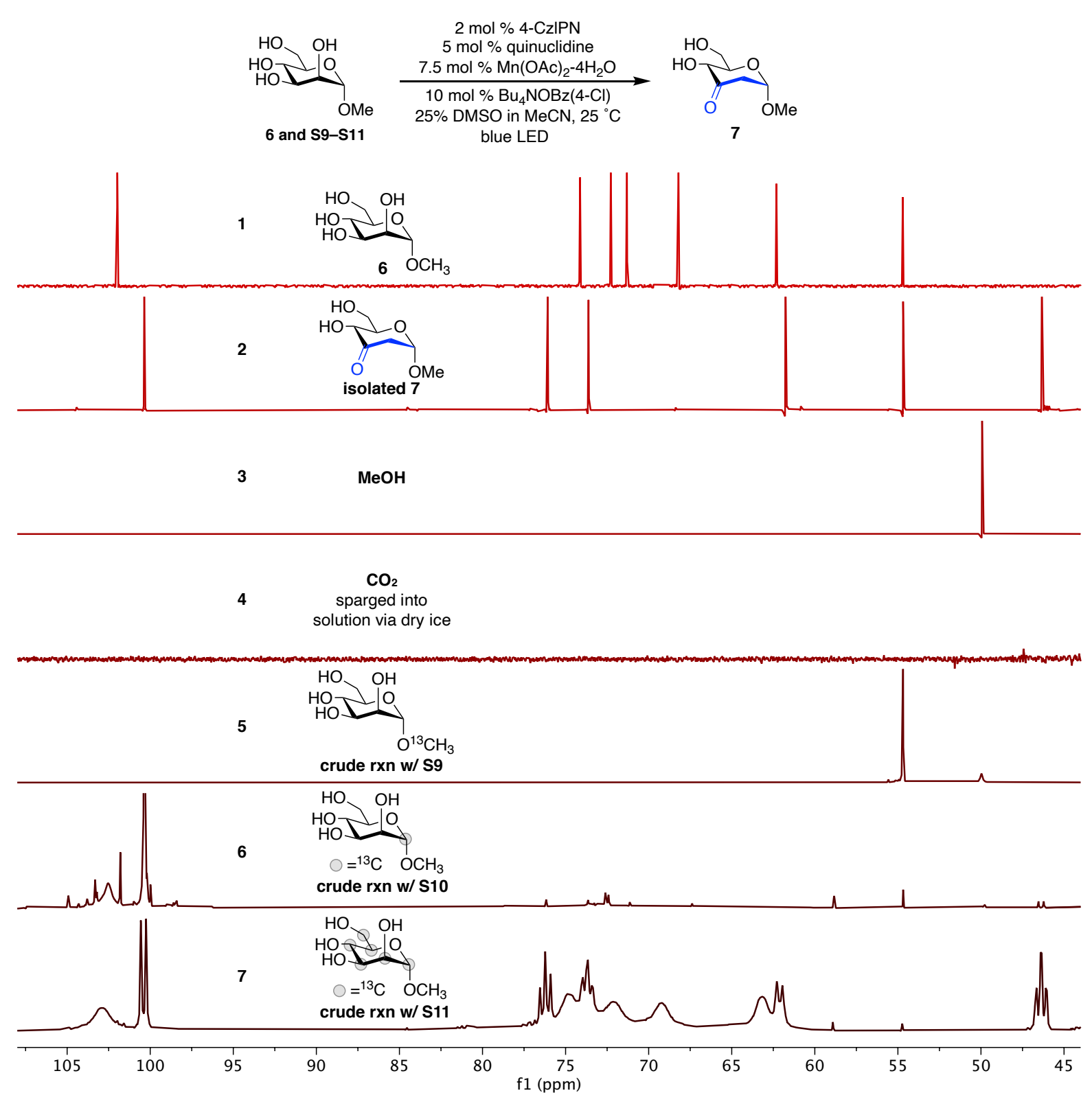

Figure S5. Same ${ }^{13} \mathrm{C}$ NMR stack as shown in Figure S4, except zoomed in on the domain between 108-44 ppm. Spectra 7 was scaled vertically to show the lack of anomeric carbons other than that in ${ }^{13} \mathrm{C}_{1}$-labeled 7. 
Overall, these findings reveal that lost mass balance arises through the catastrophic destruction of the substrate with loss of the intact aglycone, potentially with formation of volatile side-products. Two hypotheses for the source of substrate decomposition are: $\boldsymbol{a}$ ) 3-ketosugars and/or $\boldsymbol{b}$ ) other intermediates - such as those arising from the initially-formed sugar radical or unselective $\mathrm{H}$ atom abstraction.

Hypothesis $\boldsymbol{a}$ is supported by low detected mass balance when 3-keto substrates were subjected to standard reaction conditions (see SI section 8B; parts c, $\mathrm{d}$ and f). However, we cannot conclusively state whether ketone products (such as 3, 29, and 30) are formed under the reaction condition: our studies only demonstrate that ketone products are unstable and decompose under the reaction conditions.

Hypothesis $\boldsymbol{b}$ is supported by the low detected mass balance when substrates known to undergo selective $\mathrm{H}$ atom abstraction at positions not bearing an alpha, axial hydroxyl substituent were subjected to standard reaction conditions (see SI section 8A; parts b and c). However, we cannot conclusively state whether unselective $\mathrm{H}$ atom abstraction is occurring on substrates that produce deoxyketopyranoside products. Trapping studies, such as those shown in SI section 8J, did not lead to the detection of detection of adducts arising from sugar radicals (such as $\mathbf{3 1}, \mathbf{3 2}$, or others). 


\section{D) Time Course}
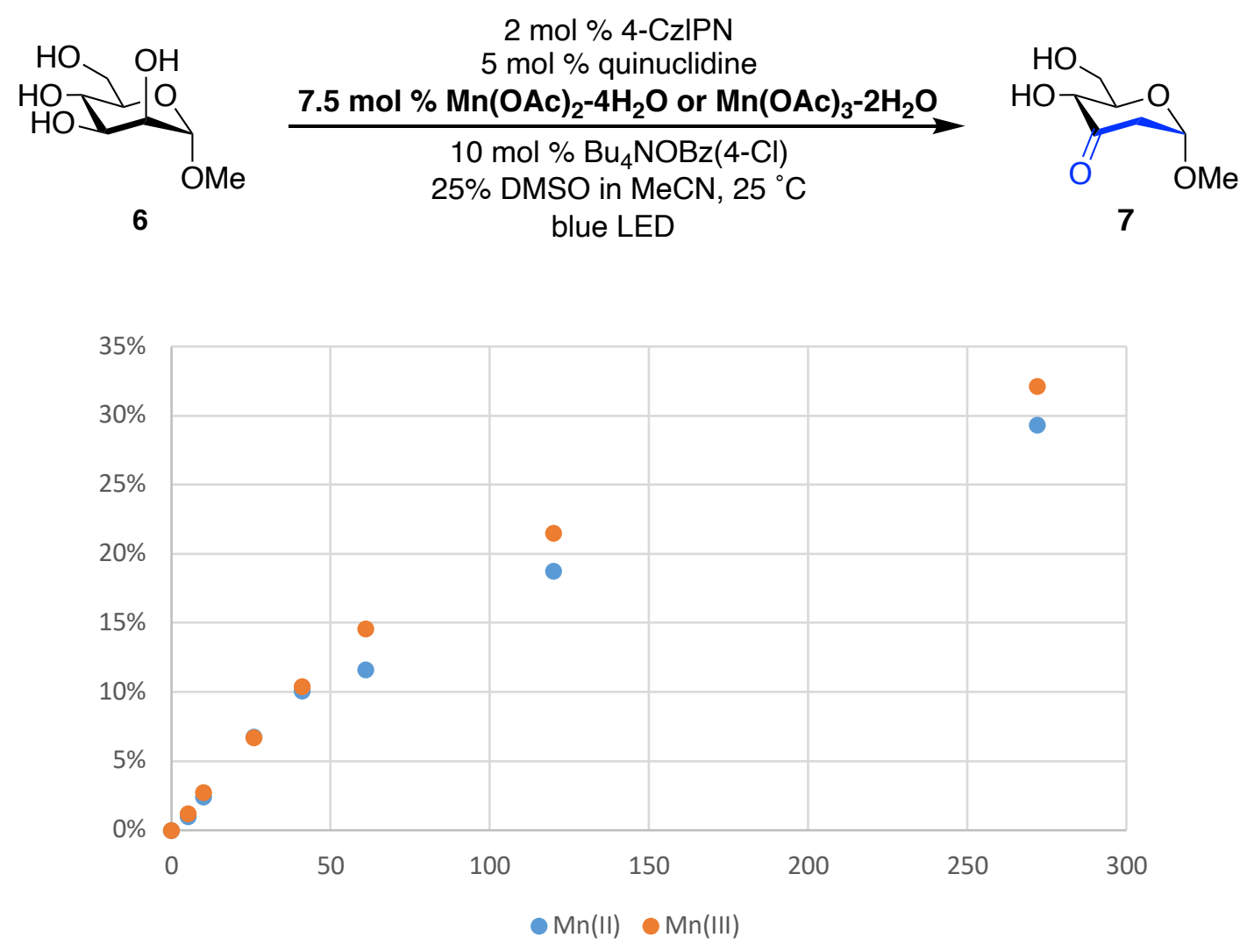

\section{E) EPR Experiments}

The reactions were set up as according to general procedure A unless indicated otherwise, such as those with $\mathrm{Mn}(\mathrm{OAc})_{3}-2 \mathrm{H}_{2} \mathrm{O}$ instead of $\mathrm{Mn}(\mathrm{OAc})_{2}-4 \mathrm{H}_{2} \mathrm{O}$ or selective removal of reaction components. Following irradiation with blue LED, the samples were brought back into the purgebox. The reactions were then diluted 10-fold with 5\% DMSO in MeCN solution to achieve $1 \mu \mathrm{M}$ manganese, placed into Wilmad $4 \mathrm{~mm}$ thin wall quartz EPR tubes, and capped with septa. Once taken out of the purge box, the samples were immediately placed into liquid $\mathrm{N}_{2}$ and analyzed by EPR.

Samples were also prepared in the same fashion with only $\mathrm{Mn}(\mathrm{OAc})_{2}-4 \mathrm{H}_{2} \mathrm{O}$ and $\mathrm{Mn}(\mathrm{OAc})_{3}-2 \mathrm{H}_{2} \mathrm{O}$ in $5 \% \mathrm{DMSO} / \mathrm{MeCN}$ for comparison (no irradiation with blue LED). Care was taken to ensure the same concentration of manganese in all samples. 

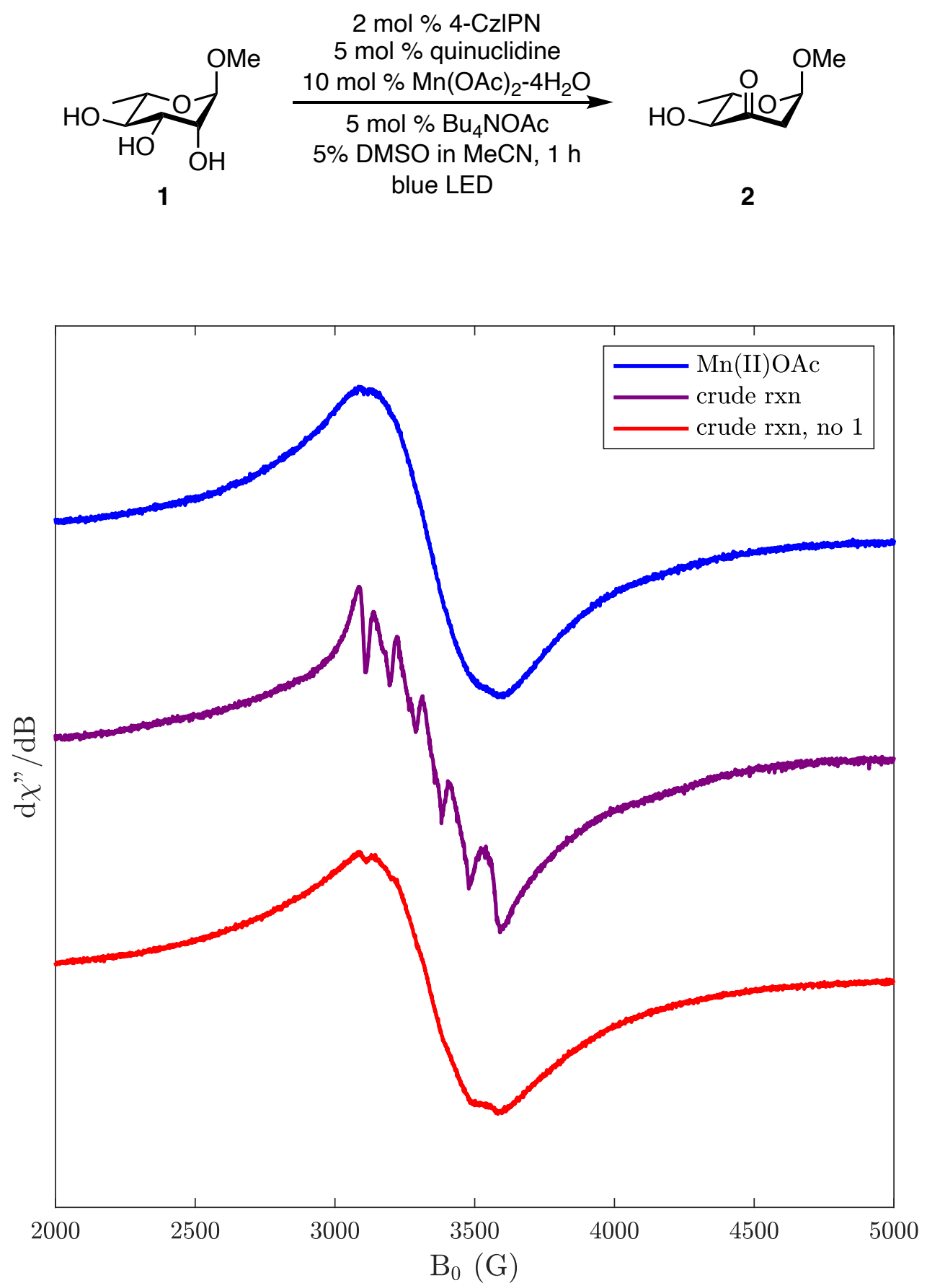

Figure S2. EPR spectra for $\mathrm{Mn}(\mathrm{OAc})_{2}-4 \mathrm{H}_{2} \mathrm{O}$ alone (no irradiation), crude rxn under standard reaction condition withs $\mathrm{Mn}(\mathrm{OAc})_{2}-4 \mathrm{H}_{2} \mathrm{O}$ (1 h irradiation), and crude rxn under standard reaction condition withs $\mathrm{Mn}(\mathrm{OAc})_{2}-4 \mathrm{H}_{2} \mathrm{O}$ without 1 (1 h irradiation). An isotropic peak is observed for $\mathrm{Mn}(\mathrm{OAc})_{2}-4 \mathrm{H}_{2} \mathrm{O}$ alone and reaction conditions without sugar. In the presence of sugar, $\mathbf{1}$, hyperfine splitting is observed, indicating a new ligand environment for $\mathrm{Mn}^{2+}$. 

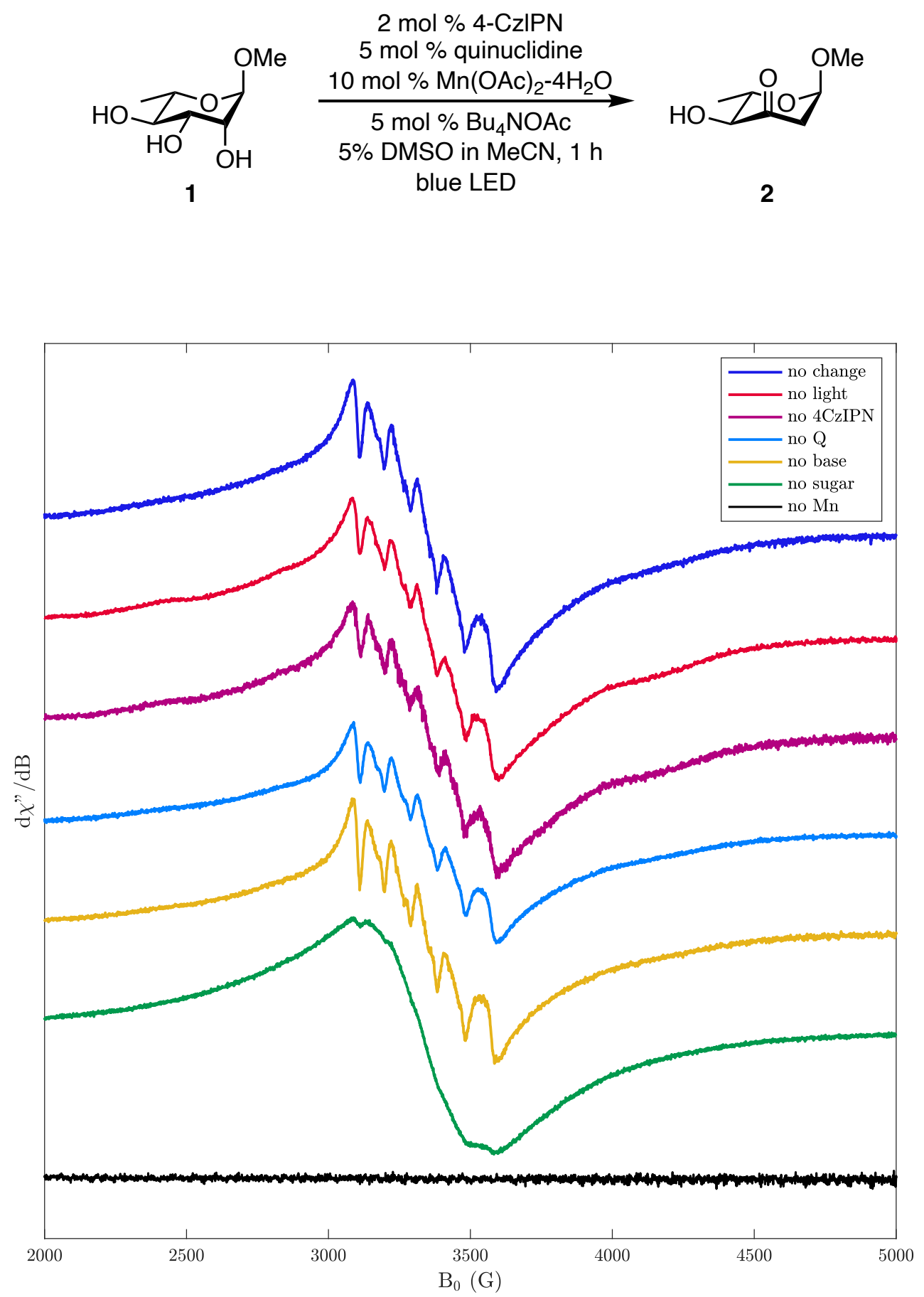

Figure S3. EPR spectra showing that sugar is the only reaction component necessary for the formatting of a hyperfine splitting pattern. All reactions were illuminated with blue LED prior to preparation of the EPR samples, except the no light control. 

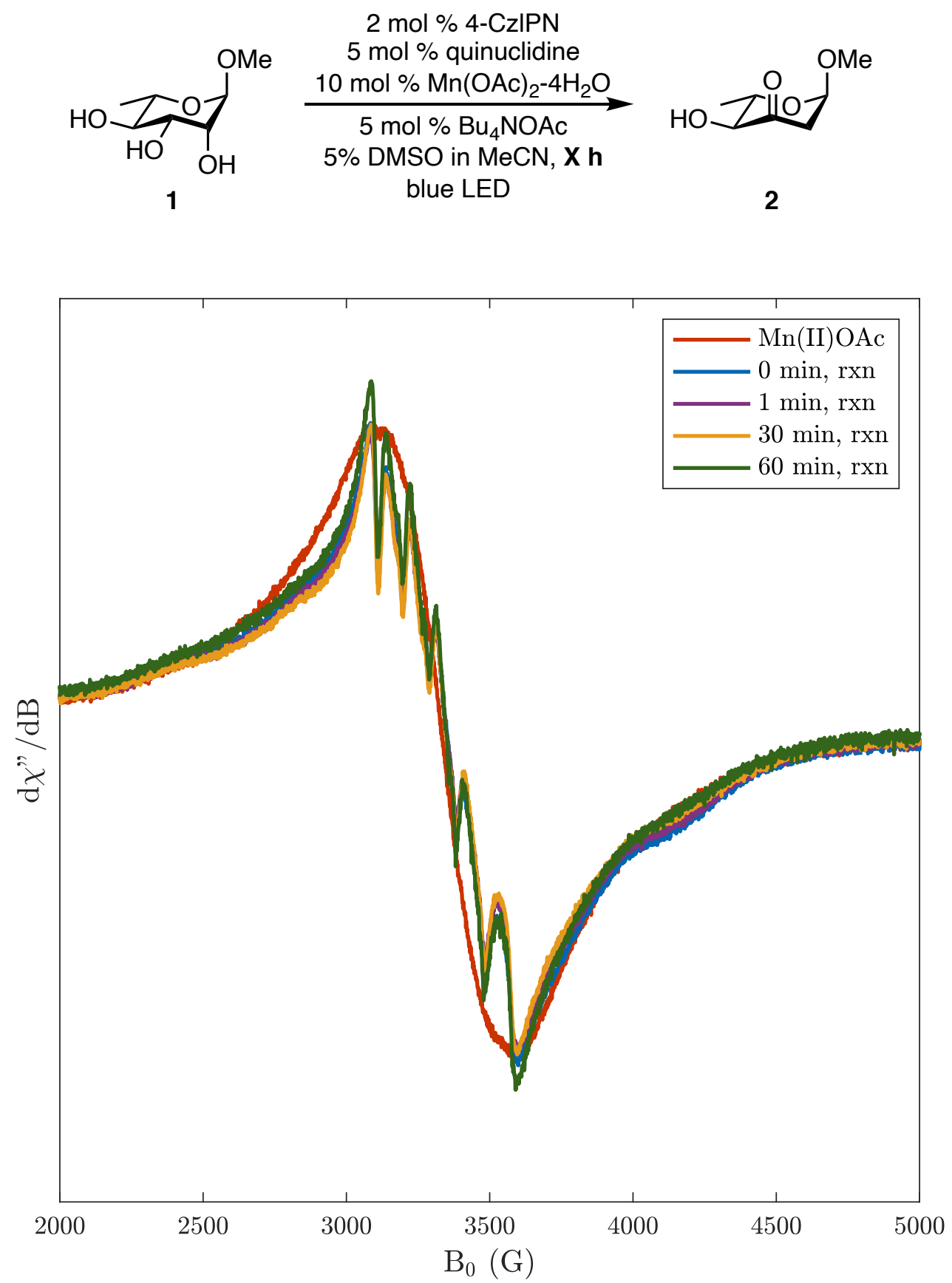

Figure S4. Overlapped EPR spectra for $\mathrm{Mn}(\mathrm{OAc})_{2}-4 \mathrm{H}_{2} \mathrm{O}$ alone (no irradiation) and standard $\mathrm{Mn}(\mathrm{OAc})_{2}-4 \mathrm{H}_{2} \mathrm{O}$ conditions at $0 \mathrm{~min}, 1 \mathrm{~min}, 30 \mathrm{~min}$, and $60 \mathrm{~min}$ of blue LED irradiation. Overall concentration of for $\mathrm{Mn}(\mathrm{OAc})_{2}-4 \mathrm{H}_{2} \mathrm{O}$ does not change with respect to reaction time. 

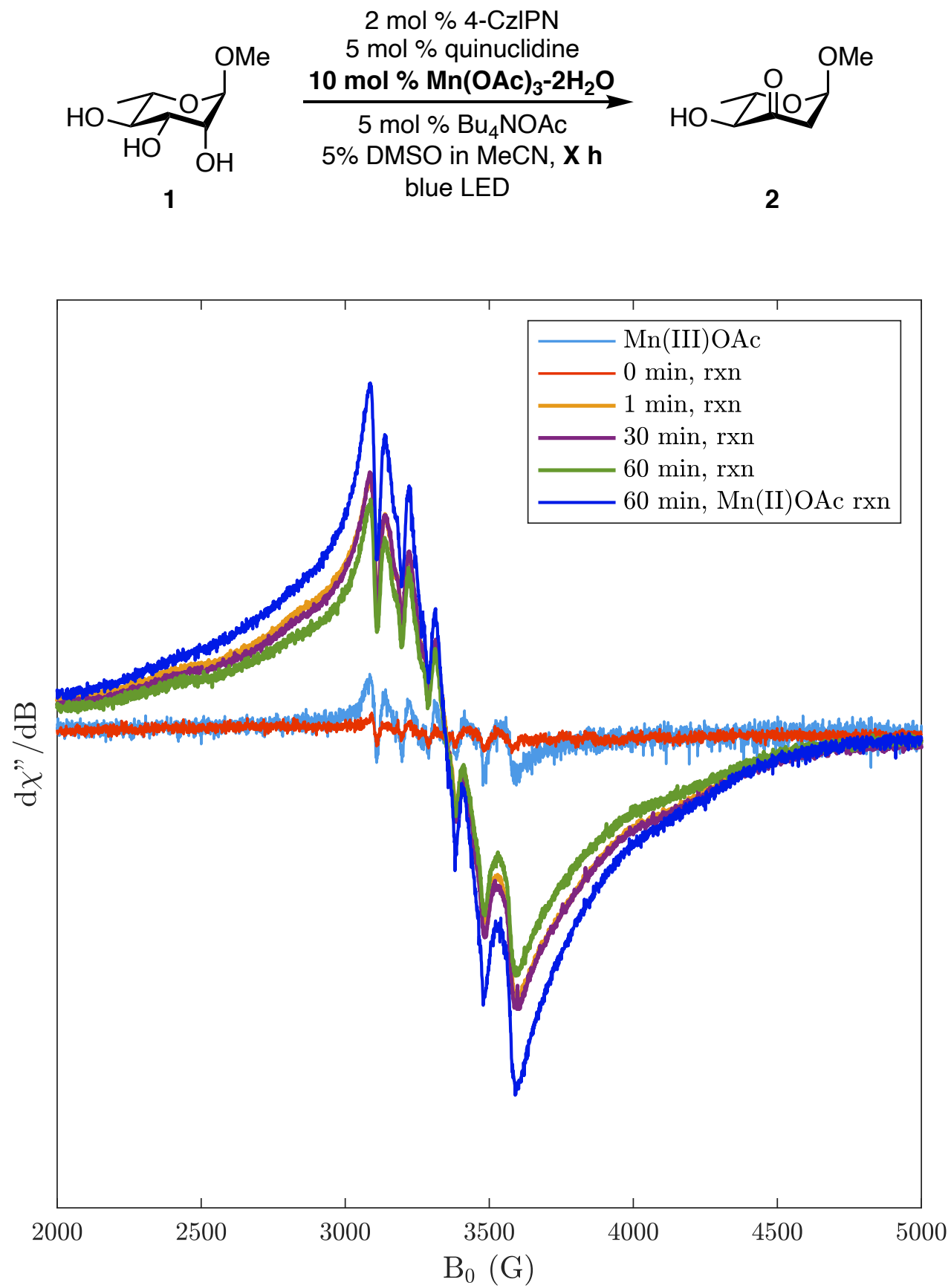

Figure S5. Overlapped EPR spectra for $\mathrm{Mn}(\mathrm{OAc})_{3}-2 \mathrm{H}_{2} \mathrm{O}$ alone (no irradiation), $\mathrm{Mn}(\mathrm{OAc})_{3}-2 \mathrm{H}_{2} \mathrm{O}$ conditions at $0 \mathrm{~min}, 1 \mathrm{~min}, 30 \mathrm{~min}$, and $1 \mathrm{~h}$ of blue LED irradiation, and standard $\mathrm{Mn}(\mathrm{OAc})_{2}-4 \mathrm{H}_{2} \mathrm{O}$ conditions at $1 \mathrm{~h}$ of blue LED irradiation. $\mathrm{Mn}^{3+}$ is rapidly converted to $\mathrm{Mn}^{2+}$ under standard reaction conditions. 


\section{F) UV-Vis Experiments}

The reactions were set up as according to general procedure A with modified conditions noted in the scheme below. An aliquot of the reaction was taken at $\mathrm{t}=0$ for $\mathrm{UV}-\mathrm{Vis}$ analysis: the solution was diluted 40-fold with 10\% DMSO in MeCN solution to achieve $50 \mu \mathrm{M}$ manganese, placed into cuvette, and capped with septa. Once taken out of the purge box, the samples were immediately analyzed by UV-Vis. The reaction vial was then sealed with a polypropylene screw cap with a bonded PTFE faced silicone liner and then removed from the purge box. The vial was then placed $5 \mathrm{~cm}$ from a blue LED Kessil lamp and stirred at $300 \mathrm{rpm}$ with a cooling fan. After 10 minutes, the vial brought back into the purge box. An aliquot was taken at $\mathrm{t}=10 \mathrm{~min}$ according to the procedure outlined above.
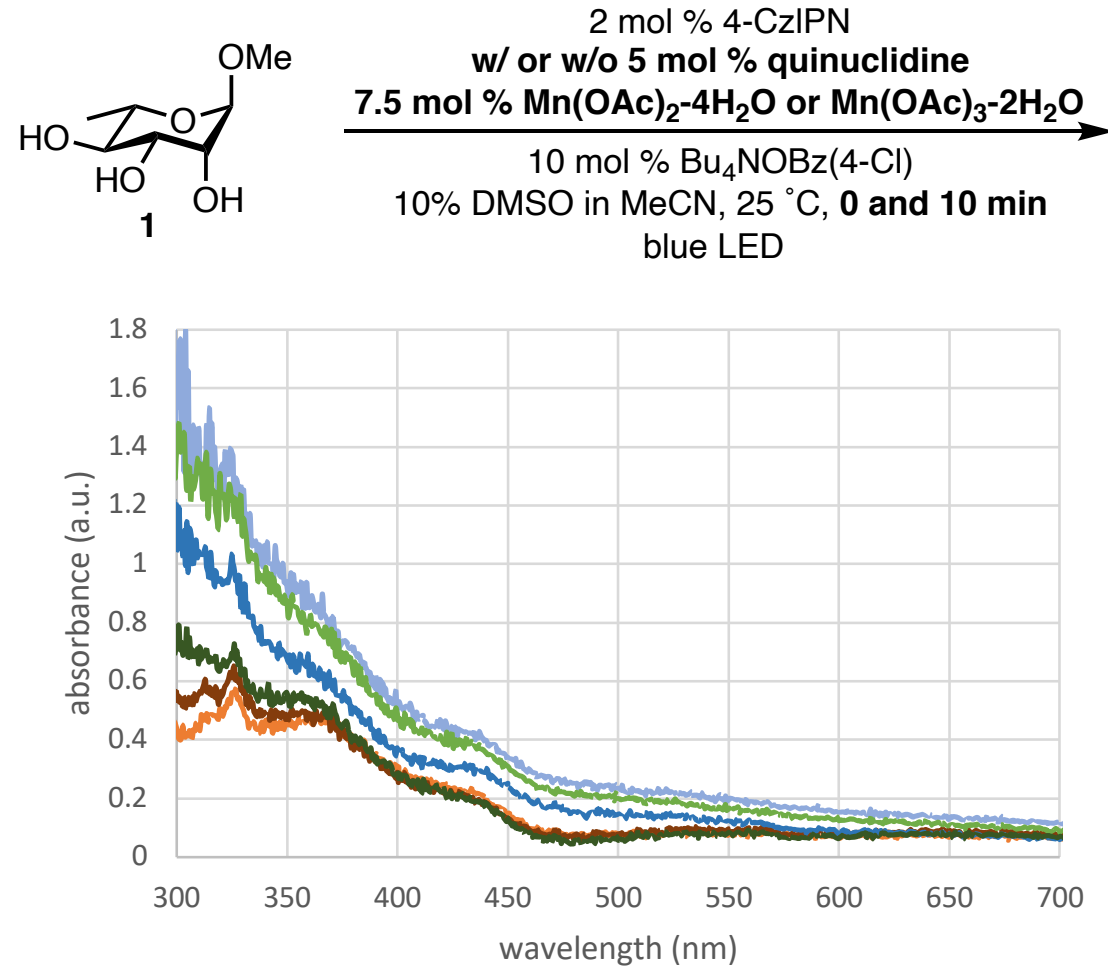

2 mol \% 4-CzIPN

w/ or w/o 5 mol \% quinuclidine $7.5 \mathrm{~mol} \% \mathrm{Mn}(\mathrm{OAc})_{2}-4 \mathrm{H}_{2} \mathrm{O}$ or $\mathrm{Mn}(\mathrm{OAc})_{3}-2 \mathrm{H}_{2} \mathrm{O}$

$10 \mathrm{~mol} \% \mathrm{Bu}_{4} \mathrm{NOBz}(4-\mathrm{Cl})$ $10 \%$ DMSO in MeCN, $25^{\circ} \mathrm{C}, 0$ and $10 \mathrm{~min}$ blue LED

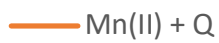

$-\mathrm{Mn}(\mathrm{II})+\mathrm{Q}$ (10 min irradiation)

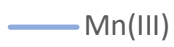

- $\mathrm{Mn}(\mathrm{III})$ (10 min irradiation)

$\longrightarrow \mathrm{Mn}(\mathrm{III})+\mathrm{Q}$

$\longrightarrow \mathrm{Mn}(\mathrm{III})+\mathrm{Q}$ (10 min irradiation)

Figure S6. UV-vis spectra of diluted reactions pre and post $10 \mathrm{~min}$ blue LED irradiation. Reduction of $\mathrm{Mn}(\mathrm{III})$ under reaction conditions (dark green trace); $\mathrm{Mn}(\mathrm{II})$ resting state. 


\section{G) Fluorescence Quenching Experiments}

Stern-Volmer fluorescence quenching experiments were performed with freshly prepared stock solutions of 4-CzIPN (1000 $\mu \mathrm{M}, \mathrm{MeCN}), \mathrm{Bu}_{4} \mathrm{NOAc}(5000 \mu \mathrm{M}, \mathrm{MeCN})$, quinuclidine (10,000 $\mu \mathrm{M}$, $\mathrm{MeCN}), \mathrm{Mn}(\mathrm{OAc})_{2}-4 \mathrm{H}_{2} \mathrm{O}(10,000 \mu \mathrm{M}, \mathrm{DMSO})$, and $\mathrm{Mn}(\mathrm{OAc})_{3}-2 \mathrm{H}_{2} \mathrm{O}(10,000 \mu \mathrm{M}$, DMSO). Samples were prepared containing the desired concentrations of reagents; the appropriate amounts of $\mathrm{MeCN}$ and DMSO were added to make $2.0 \mathrm{~mL}$ solutions of $25 \% \mathrm{DMSO}$ in $\mathrm{MeCN}$. For each sample, the luminescence was acquired two times and averaged.

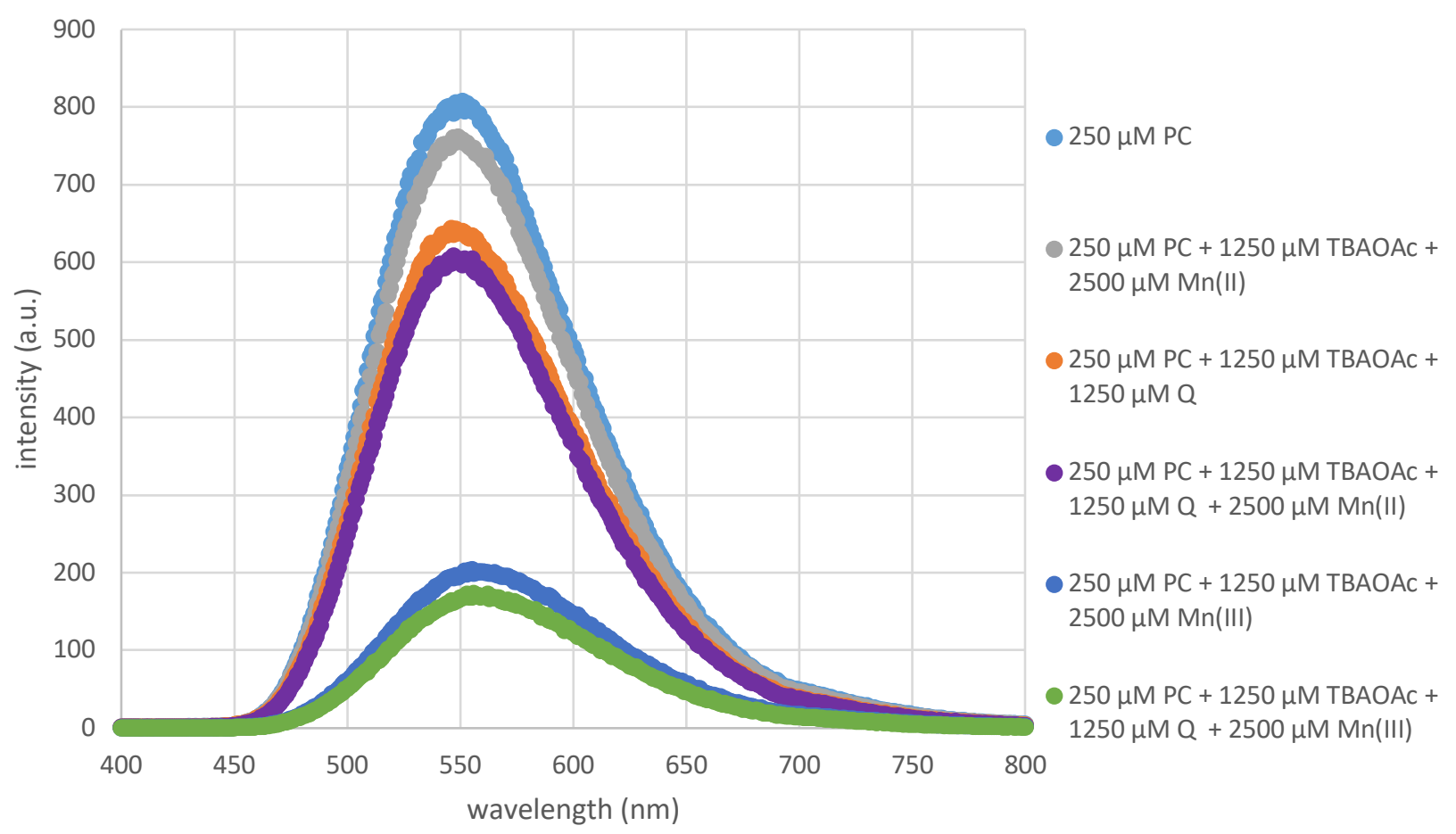

Figure S7. The emission intensity changes of $4 \mathrm{CzIPN}$ in the presence of different reaction components. 


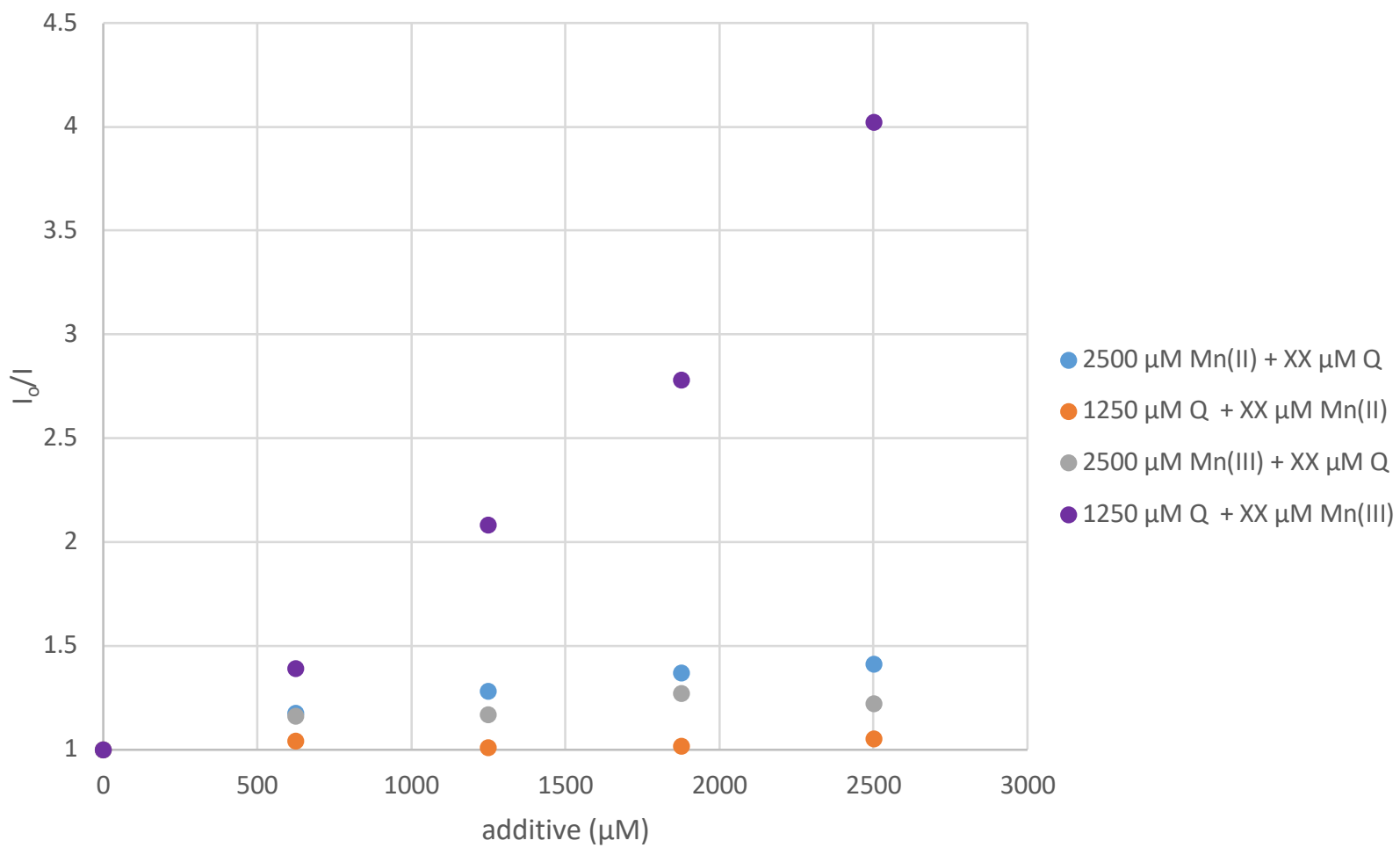

Figure S8. Stern-Volmer plot of 4CzIPN with varying reaction components. All samples contained $250 \mu \mathrm{M}$ PC and $1250 \mu \mathrm{M}$ TBAOAc. 


\section{H) Binding Studies}

Samples for the NMR binding studies were prepared in the purge box with degassed $\mathrm{d}_{6}$-DMSO and $\mathrm{d}_{3}-\mathrm{MeCN}$. A stock solution of methylrhamnoside 1 was prepared in $20 \% \mathrm{~d}_{6}$-DMSO in $\mathrm{d}_{3}$ $\mathrm{MeCN}(50 \mathrm{mM}, 4500 \mu \mathrm{L})$ with $8 \mu \mathrm{L}$ benzene. The corresponding amount of [Mn] was weighed into an NMR tube and brought into the purge box. $500 \mu \mathrm{L}$ of the sugar stock solution was added to each tube. The tubes were capped with septa, electrical taped, and brought out of the purge box. The higher concentration samples were sonicated and slightly heated to dissolve all [Mn]. The sample were allowed to sit for $1 \mathrm{~h}$ prior to ${ }^{1} \mathrm{H}$ NMR analysis. The spectra were referenced to the benzene peak, as both $\mathrm{MeCN}$ and DMSO exhibited shifting with respect to [Mn].
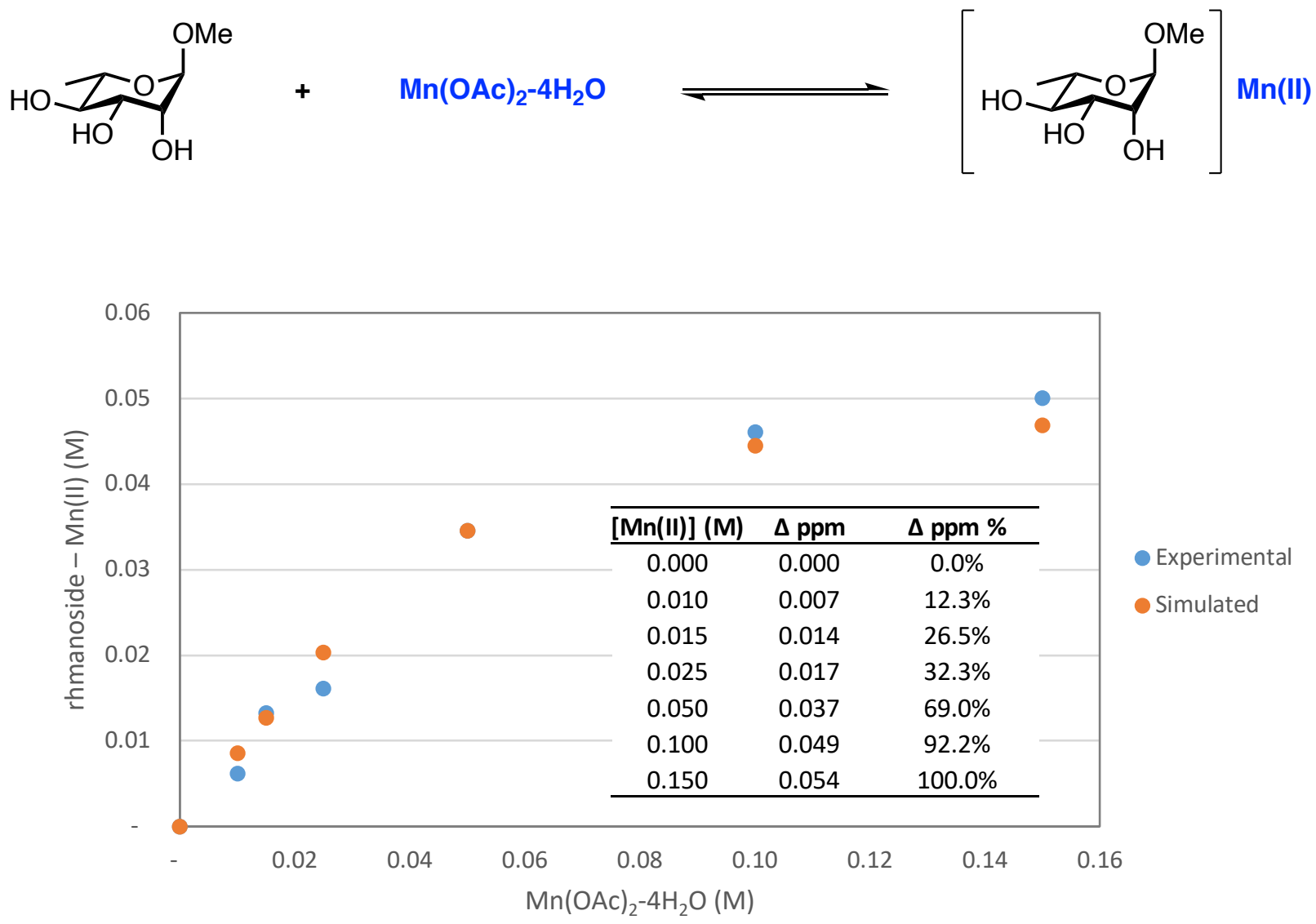

Figure S9. Equilibrium binding curve between $\mathrm{Mn}$ (II) and methylrhamnoside. Tracking peak shift of $-\mathrm{OMe}, \mathrm{K}=145 \mathrm{M}^{-1}$. 

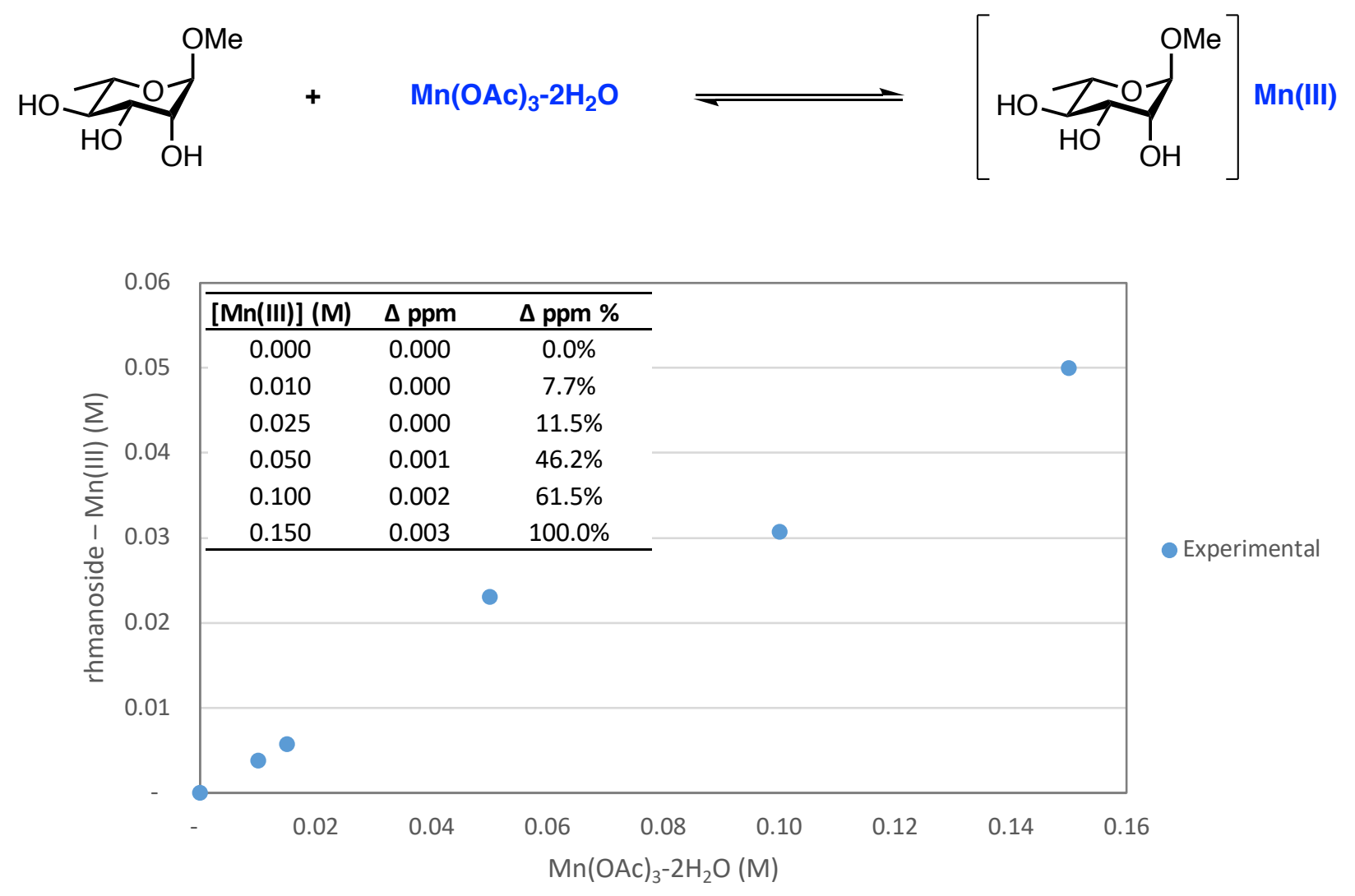

Figure S10. Equilibrium binding curve between $\mathrm{Mn}(\mathrm{III})$ and methyl rhamnoside. Tracking peak shift of -OMe, $\mathrm{K}=$ n.d. $\mathrm{M}^{-1}$. Tracking the peak shift of -Me resulted in no measurable binding. 


\section{I) Cyclic Voltammetry}

Cyclic voltammetry (CV) experiments were conducted in a $25 \mathrm{~mL}$ 3-neck round bottom fitted with a glassy carbon working electrode ( $3 \mathrm{~mm}$ in diameter, $\mathrm{BASi}$ ), an $\mathrm{Ag} / \mathrm{AgNO}_{3}$ reference electrode $\left(100 \mathrm{mM} \mathrm{TBABF}_{4}\right.$ and $10 \mathrm{mM} \mathrm{AgNO}_{3}$ ), a platinum wire counter electrode, and a stir bar. The solution of interest was sparged with nitrogen for 3-5 minutes before data collection. Scan rates and potential ranges of the acquired spectra are noted in the caption. Upon completion of data gathering, ferrocene was added ( $10 \mathrm{mM}$ final concentration), to reference the potentials vs $\mathrm{Fc} / \mathrm{Fc}^{+}$. To convert the potentials from $\mathrm{Fc} / \mathrm{Fc}^{+}$to $\mathrm{SCE}, 380 \mathrm{mV}$ were added to the measured values. ${ }^{24,25}$

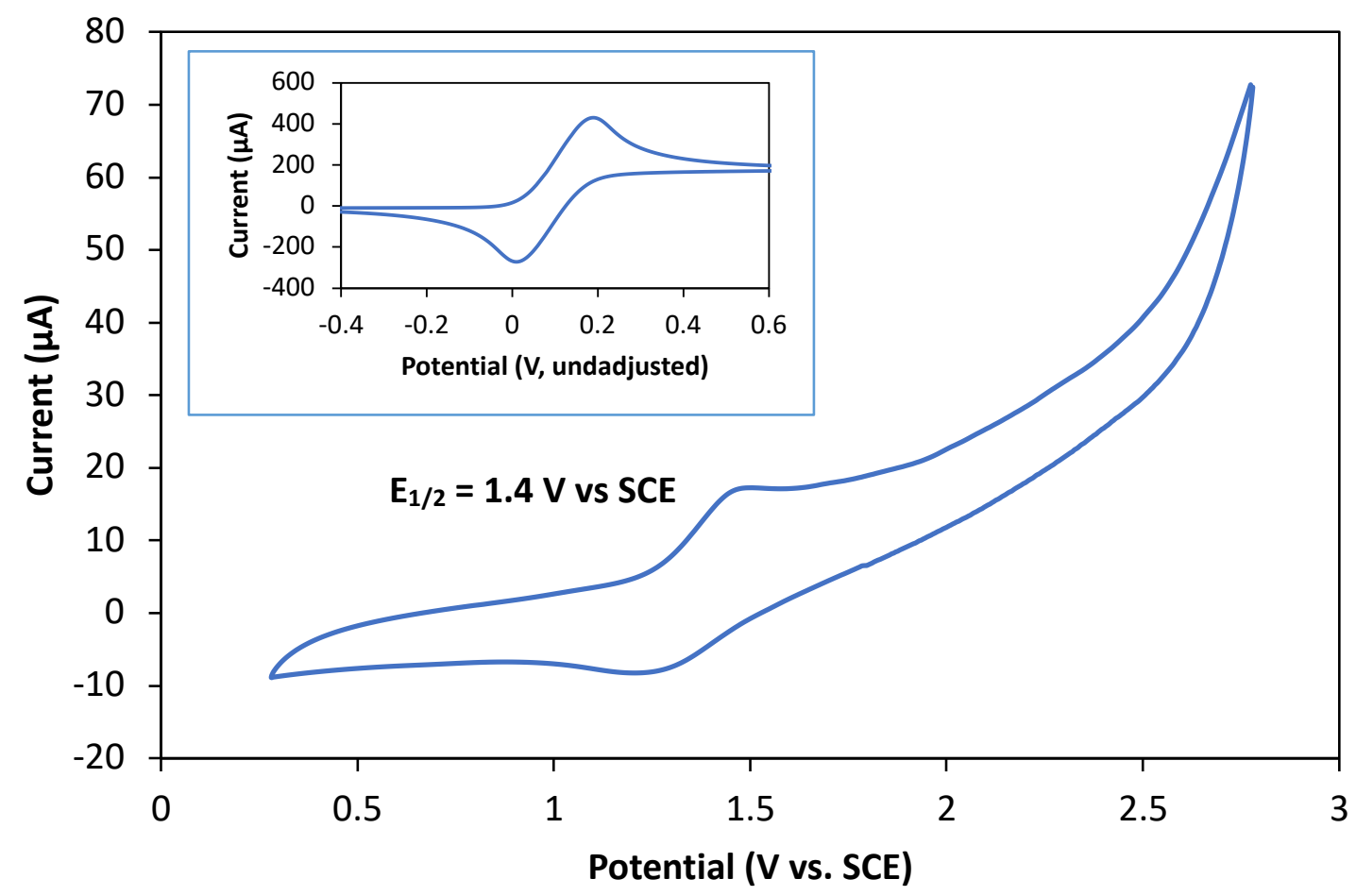

Figure S11. Cyclic voltammogram of $\mathrm{Mn}(\mathrm{OAc})_{3}-2 \mathrm{H}_{2} \mathrm{O}(10 \mathrm{mM})$ and $100 \mathrm{mM} \mathrm{TBABF}_{4}$ in $\mathrm{MeCN}_{\text {. }}$ Not fully soluble. Scanned from $2.8 \mathrm{~V}$ to $0.3 \mathrm{~V}$ and back, $100 \mathrm{mV} / \mathrm{s}$. Ferrocene $(10 \mathrm{mM})$ was added after acquiring the spectra of $\mathrm{Mn}(\mathrm{OAc})_{3}-2 \mathrm{H}_{2} \mathrm{O}$, see inset. 


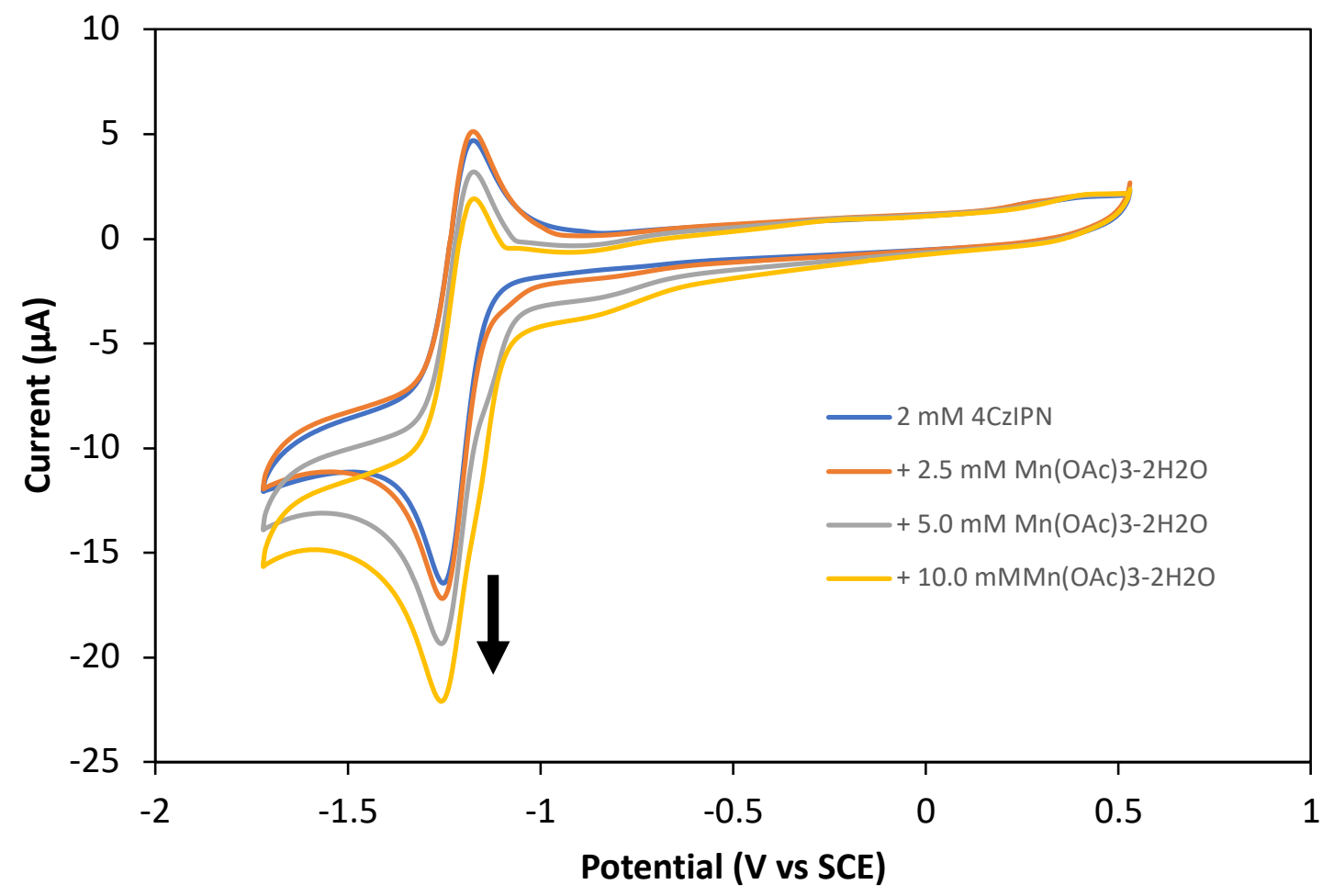

Figure S12. Cyclic voltammogram of 4CzIPN (2 mM), $\mathrm{Mn}(\mathrm{OAc})_{3}-2 \mathrm{H}_{2} \mathrm{O}(0 \mathrm{mM}, 2.5 \mathrm{mM}, 5 \mathrm{mM}$, and $10 \mathrm{mM}$ ), and $100 \mathrm{mM} \mathrm{TBABF}_{4}$ in $\mathrm{MeCN}$. 


\section{J) Trapping Studies}

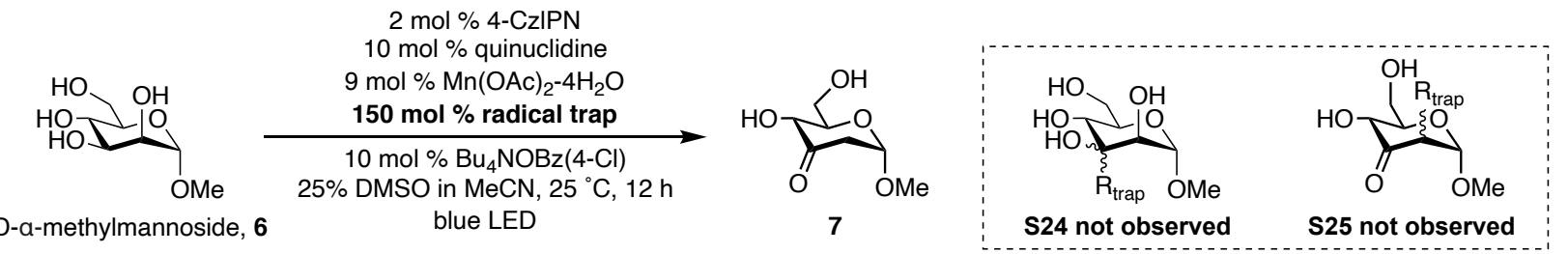

Table S6. No trapping of the proposed radical intermediates observed.

\begin{tabular}{clccc}
\hline Entry & \multicolumn{1}{c}{ Radical Trap } & Yield 7 & RSM (\%) & Notes \\
\hline 1 & None & 35 & 19 & \\
2 & Phenyl vinyl sulfone & 22 & 24 & \\
3 & Ethyl vinyl ether & 0 & 62 & \\
4 & 2-methoxypropene & 0 & 85 & \\
5 & Vinylcyclohexane & 13 & 66 & \\
6 & TEMPO & 0 & 20 & 18\% other, present w/o quinucldine \\
8 & Galvinoxyl & 0 & 92 & \\
9 & PhSe-SePh & 0 & 74 & \\
10 & Selectflour & 0 & 52 & \\
\hline
\end{tabular}




\section{References}

(1) Kawamata, Y.; Yan, M.; Liu, Z.; Bao, D. H.; Chen, J.; Starr, J. T.; Baran, P. S. Scalable, Electrochemical Oxidation of Unactivated C-H Bonds. J. Am. Chem. Soc. 2017, 139 (22), 7448-7451.

(2) Ma, Z.; Lu, J.; Wang, X.; Chen, C. Revisiting the Kinnel-Scheuer Hypothesis for the Biosynthesis of Palau'amine. Chem. Commun. 2011, 47 (1), 427-429.

(3) Luo, J.; Zhang, J. Donor-Acceptor Fluorophores for Visible-Light-Promoted Organic Synthesis: Photoredox/Ni Dual Catalytic C(Sp3)-C(Sp2) Cross-Coupling. ACS Catal. 2016, 6 (2), 873-877.

(4) Binkley, R. W.; Goewey, G. S.; Johnston, J. C. Regioselective Ring Opening of Selected Benzylidene Acetals. A Photochemically Inititated Reaction for Partial Deprotection of Carbohydrates. J. Org. Chem. 1984, 49 (6), 992-996.

(5) Nakae, K.; Kurata, I.; Kojima, F.; Igarashi, M.; Hatano, M.; Sawa, R.; Kubota, Y.; Adachi, H.; Nomoto, A. Sacchathridine A, a Prostaglandin Release Inhibitor from Saccharothrix Sp. J. Nat. Prod. 2013, 76 (4), 720-722.

(6) Grayson, E. J.; Bernardes, G. J. L.; Chalker, J. M.; Boutureira, O.; Koeppe, J. R.; Davis, B. G. A Coordinated Synthesis and Conjugation Strategy for the Preparation of Homogeneous Glycoconjugate Vaccine Candidates. Angew. Chemie - Int. Ed. 2011, 50 (18), 4127-4132.

(7) Fulse, D. B.; Heung, B. J.; Kwan, S. K. Synthesis of Tri-, Hexa-, and Nonasaccharide Subunits of the Atypical O-Antigen Polysaccharide of the Lipopolysaccharide from Danish Helicobacter Pylori Strains. J. Org. Chem. 2007, 72 (26), 9963-9972.

(8) Jalsa, N. K. Regioselective Removal of the Anomeric O-Benzyl from Differentially Protected Carbohydrates. Tetrahedron Lett. 2011, 52 (49), 6587-6590.

(9) Boissière-Junot, N.; Tellier, C.; Rabiller, C. On the Regioselective Acylation of 1,6Anhydro- $\beta$-D- and L-Hexopyranoses Catalysed by Lipases: Structural Bass and Synthetic Applications. J. Carbohydr. Chem. 1998, 17 (1), 99-115.

(10) Dimakos, V.; Liu, J. J. W.; Ge, Z.; Taylor, M. S. Copper-Mediated Anomeric O-Arylation with Organoboron Reagents. Org. Biomol. Chem. 2019, 17 (23), 5671-5674.

(11) Quibell, M.; Schulz-Utermoehl, T.; Murray, F. ANTIBACTERIAL ANTISENSE AGENTS Le. WO2020109792A1, 2020.

(12) Fisher, M. J.; Myers, C. D.; Joglar, J.; Chen, S. H.; Danishefsky, S. J. Synthetic Studies toward Rapamycin: A Solution to a Problem in Chirality Merger through Use of the Ireland Reaction. J. Org. Chem. 1991, 56 (20), 5826-5834.

(13) Lee, D.; Taylor, M. S. Borinic Acid-Catalyzed Regioselective Acylation of Carbohydrate Derivatives. J. Am. Chem. Soc. 2011, 133 (11), 3724-3727.

(14) Gong, J.; Li, W.; Fu, P.; MacMillan, J.; De Brabander, J. K. Isolation, Structure, and Total 
Synthesis of the Marine Macrolide Mangrolide D. Org. Lett. 2019, 21 (8), 2957-2961.

(15) Mallams, A. K.; Puar, M. S.; Rossman, R. R.; McPhail, A. T.; Macfarlane, R. D.; Stephens, R. L. Kijanimicin. Part 3. Structure and Absolute Stereochemistry of Kijanimicin. J. Chem. Soc. Perkin Trans. 1 1983, 1497-1534.

(16) Grethe, G.; Mitt, T.; Williams, T. H.; Uskokovic, M. R. Synthesis of Daunosamine. J. Org. Chem. 1983, 48 (26), 5309-5315.

(17) Evtushenko, E. V; Ovodov, Y. S. Synthesis of Methyl 2,6- and 3,6-Dideoxy- $\alpha$-L-ArabinoHexopyranosides and Methyl 4,6-Dideoxy- $\alpha$-L-Lyxo-Hexopyranoside. Chem. Nat. Compd. 1990, 26 (3), 263-264.

(18) Zeng, J.; Sun, G.; Yao, W.; Zhu, Y.; Wang, R.; Cai, L.; Liu, K.; Zhang, Q.; Liu, X.-W.; Wan, Q. 3-Aminodeoxypyranoses in Glycosylation: Diversity-Oriented Synthesis and Assembly in Oligosaccharides. Angew. Chemie Int. Ed. 2017, 56 (19), 5227-5231.

(19) Leet, J. E.; Schroeder, D. R.; Langley, D. R.; Colson, K. L.; Huang, S.; Klohr, S. E.; Lee, M. S.; Golik, J.; Hofstead, S. J. Chemistry and Structure Elucidation of the Kedarcidin Chromophore. J. Am. Chem. Soc. 1993, 115 (18), 8432-8443.

(20) Wang, Y.; Carder, H. M.; Wendlandt, A. E. Synthesis of Rare Sugar Isomers through SiteSelective Epimerization. Nature 2020, 578 (7795).

(21) Gorelik, D. J.; Turner, J. A.; Virk, T. S.; Foucher, D. A.; Taylor, M. S. Site-and Stereoselective C-H Alkylations of Carbohydrates Enabled by Cooperative Photoredox, Hydrogen Atom Transfer, and Organotin Catalysis Scheme 1. Approaches for Substrate Activation in Site-Selective C-H Alkylations of Glycosides. Org. Lett 2021, 23, 27.

(22) Chim, I.; Wan, S.; Witte, M. D.; Minnaard, A. J.; Li, R.; Chemcomm, /; Communication, C. Site-Selective Carbon-Carbon Bond Formation in Unprotected Monosaccharides Using Photoredox Catalysis †. Chem. Commun 2017, 4926, 4926.

(23) Chung, K.; M. Waymouth, R. Selective Catalytic Oxidation of Unprotected Carbohydrates. ACS Catal. 2016, 6 (7), 4653-4659.

(24) G. Yayla, H.; Wang, H.; T. Tarantino, K.; S. Orbe, H.; R. Knowles, R. Catalytic RingOpening of Cyclic Alcohols Enabled by PCET Activation of Strong O-H Bonds. J. Am. Chem. Soc. 2016, 138 (34), 10794-10797.

(25) Pavlishchuk, V. V.; Addison, A. W. Conversion Constants for Redox Potentials Measured versus Different Reference Electrodes in Acetonitrile Solutions at $25^{\circ} \mathrm{C}$. Inorganica Chim. Acta 2000, 298 (1), 97-102. 
S3

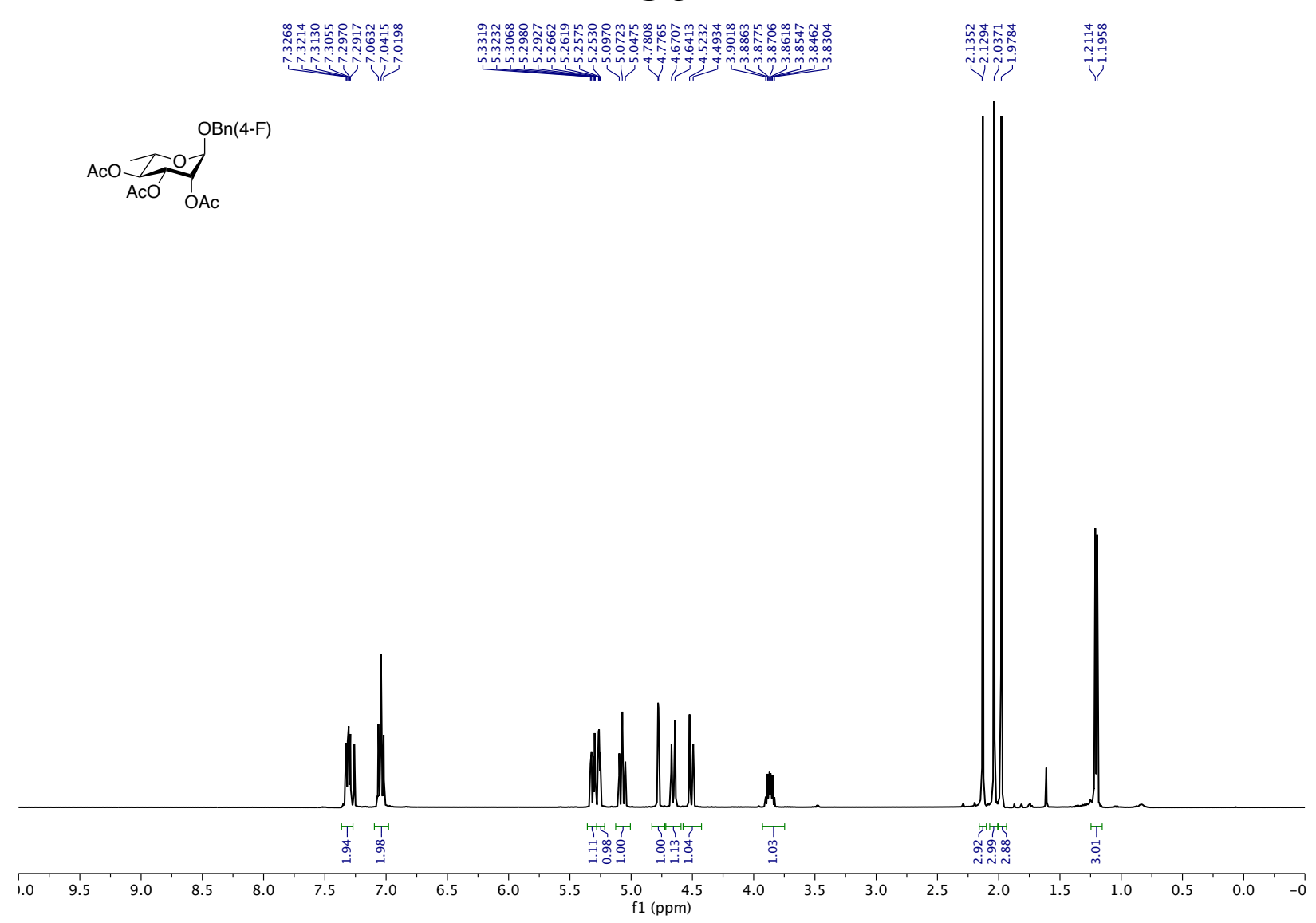

S3

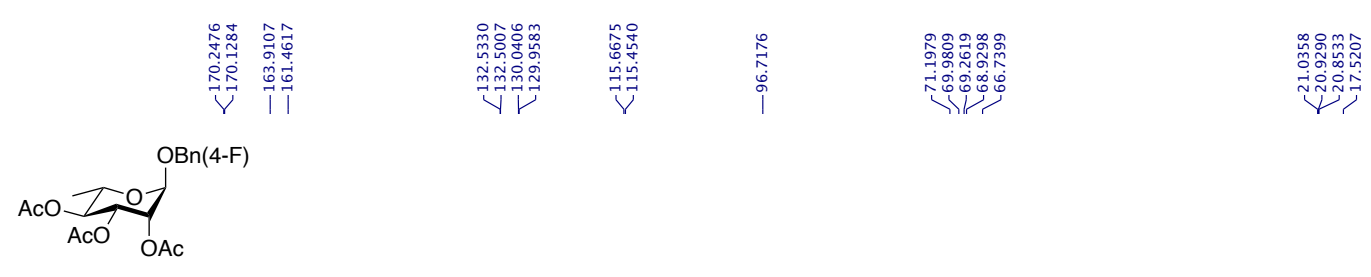

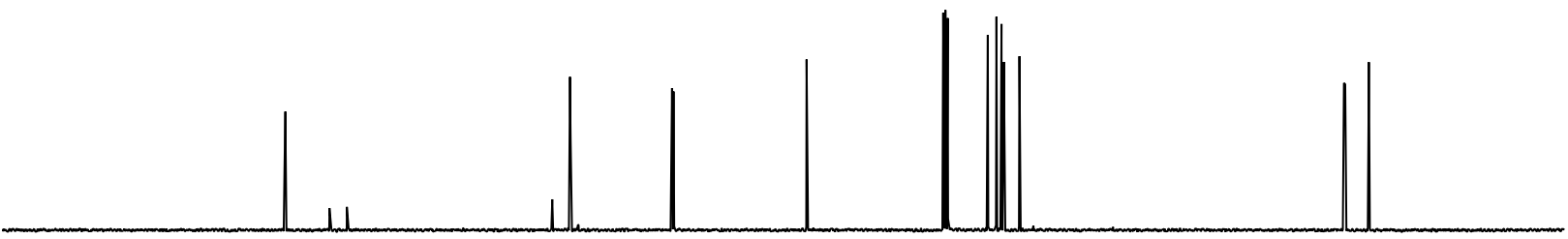

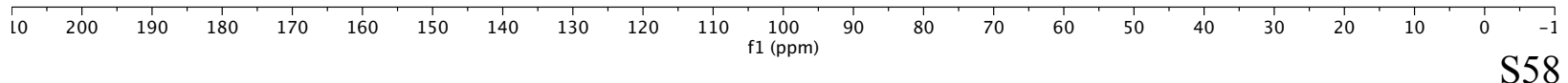




\section{S3}

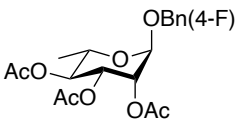
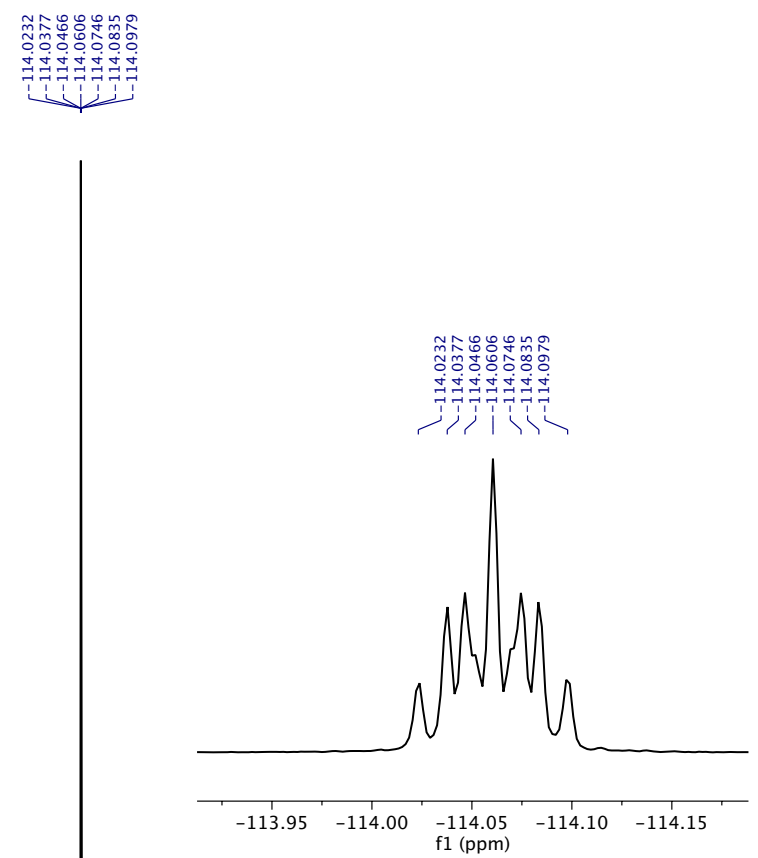

\begin{tabular}{lllllllllllllllllllllll}
\hline 0 & 0 & -10 & -20 & -30 & -40 & -50 & -60 & -70 & -80 & -90 & -100 & -110 & -120 & -130 & -140 & -150 & -160 & -170 & -180 & -190 & -200 & -2
\end{tabular}

\section{S6}

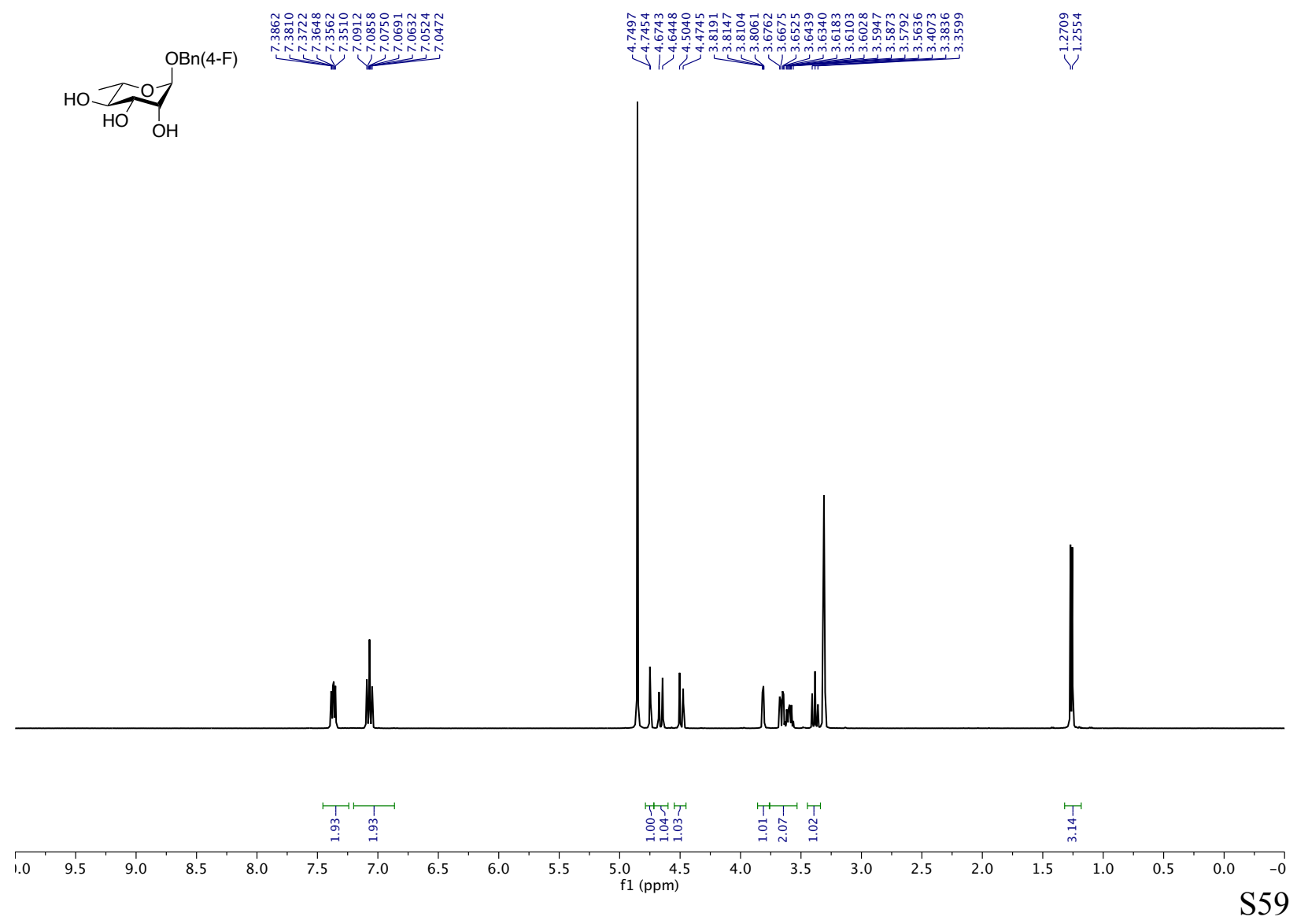




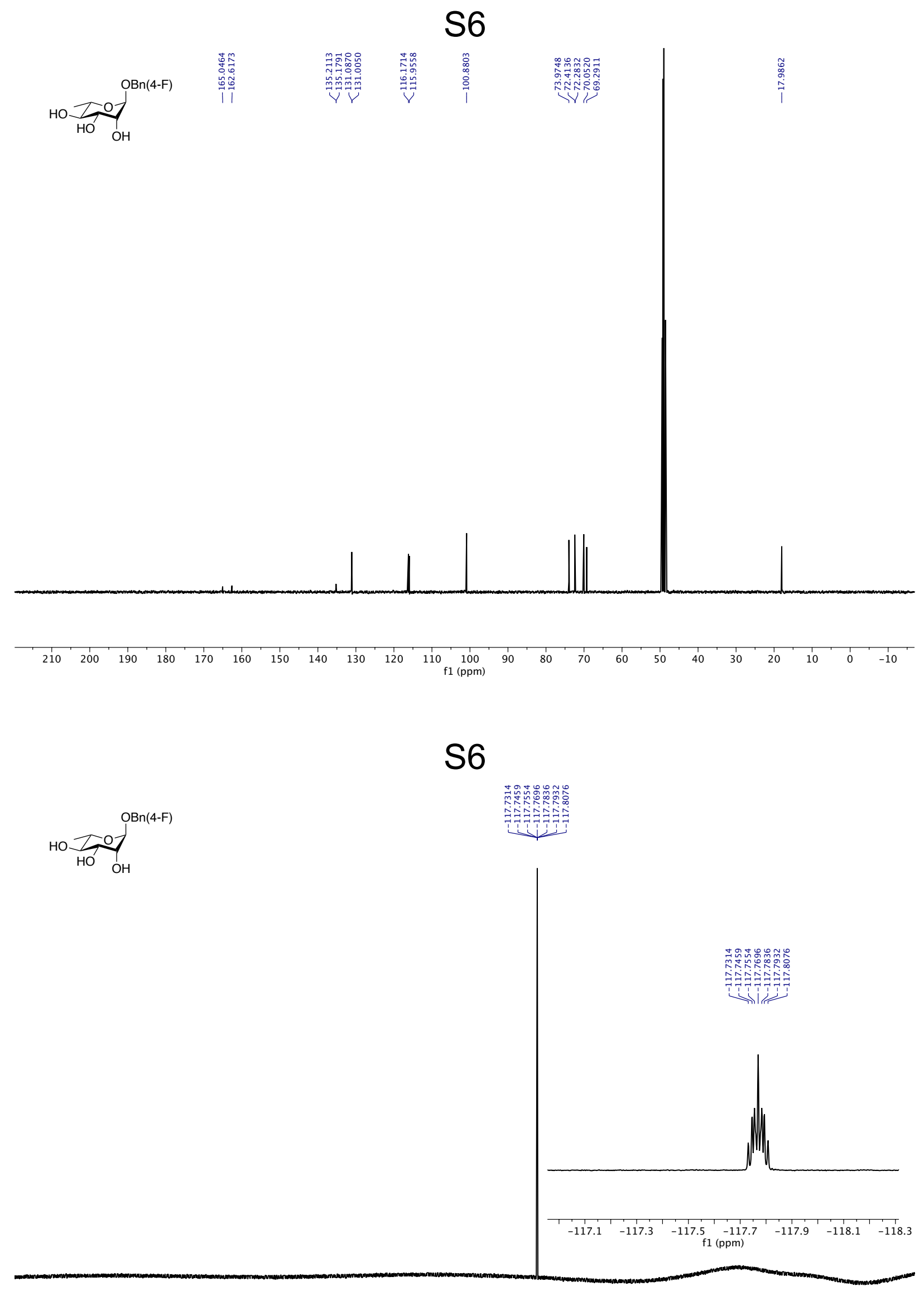

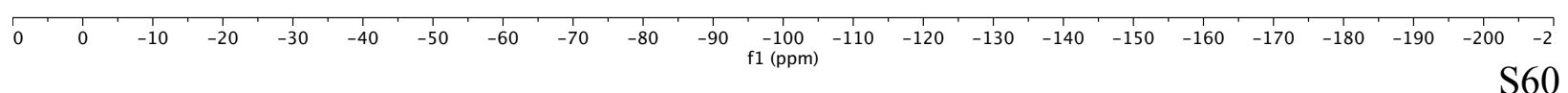




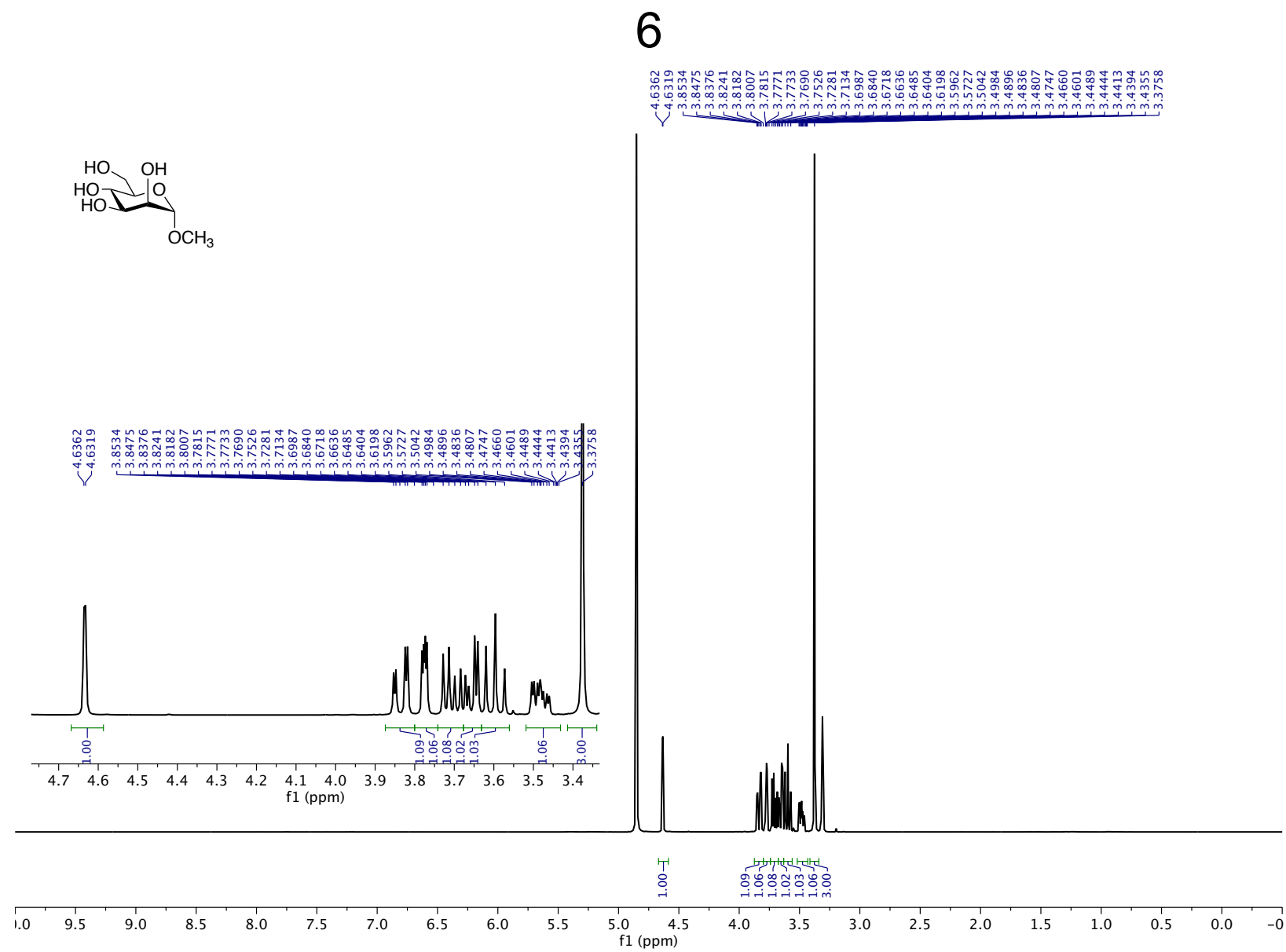

6

$\underbrace{\mathrm{HO}}_{\mathrm{OCH}_{3}}$
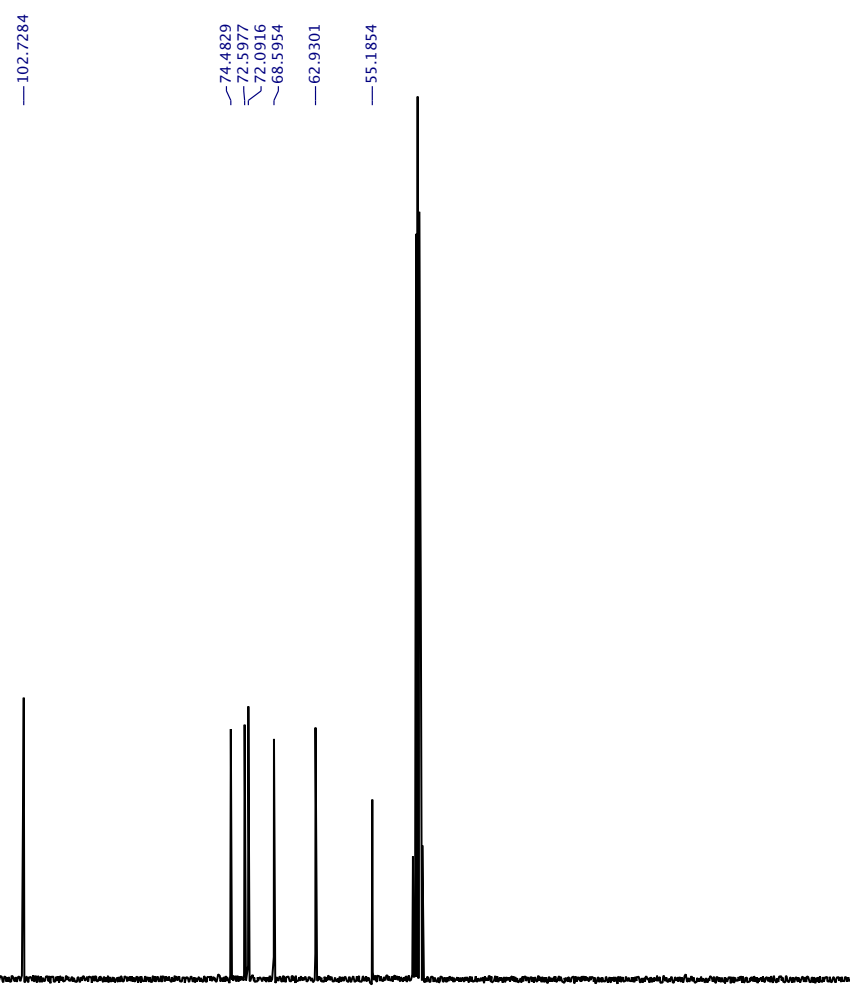

\begin{tabular}{rrrrrrrrrrrrrrrrrrrrrrr}
\hline 10 & 200 & 190 & 180 & 170 & 160 & 150 & 140 & 130 & 120 & 110 & $\begin{array}{c}100 \\
\mathrm{ff}(\mathrm{ppm})\end{array}$ & 90 & 80 & 70 & 60 & 50 & 40 & 30 & 20 & 10 & 0 & -1 \\
S61
\end{tabular} 
S9



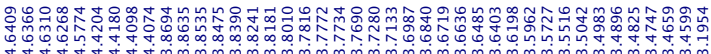

ن

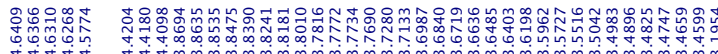

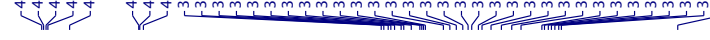

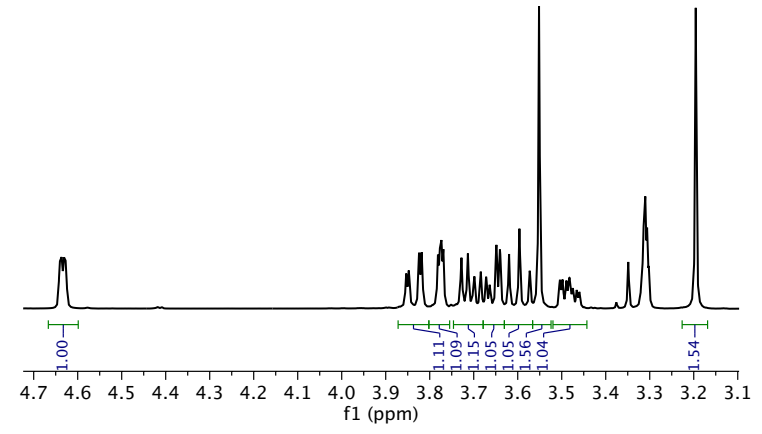

S9



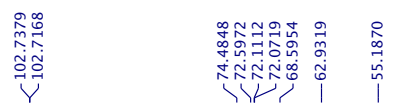

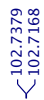

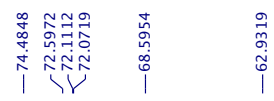
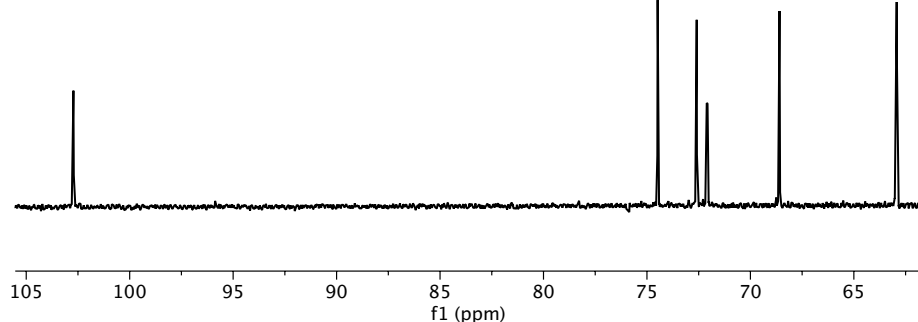

$10 \quad 200$

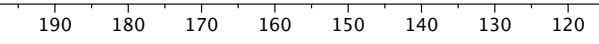

$10 \begin{gathered}100 \\ f 1(p p m)\end{gathered}$

$80 \quad 70$

$50 \quad 40$

$30 \quad 20$

$\begin{array}{lll}10 & 0 & -1\end{array}$ 


\section{S10}

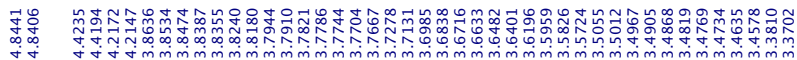

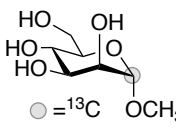

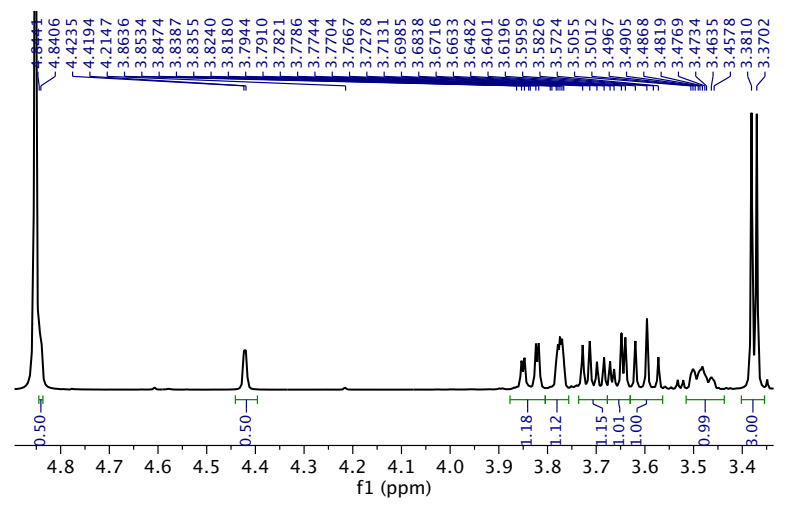

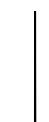
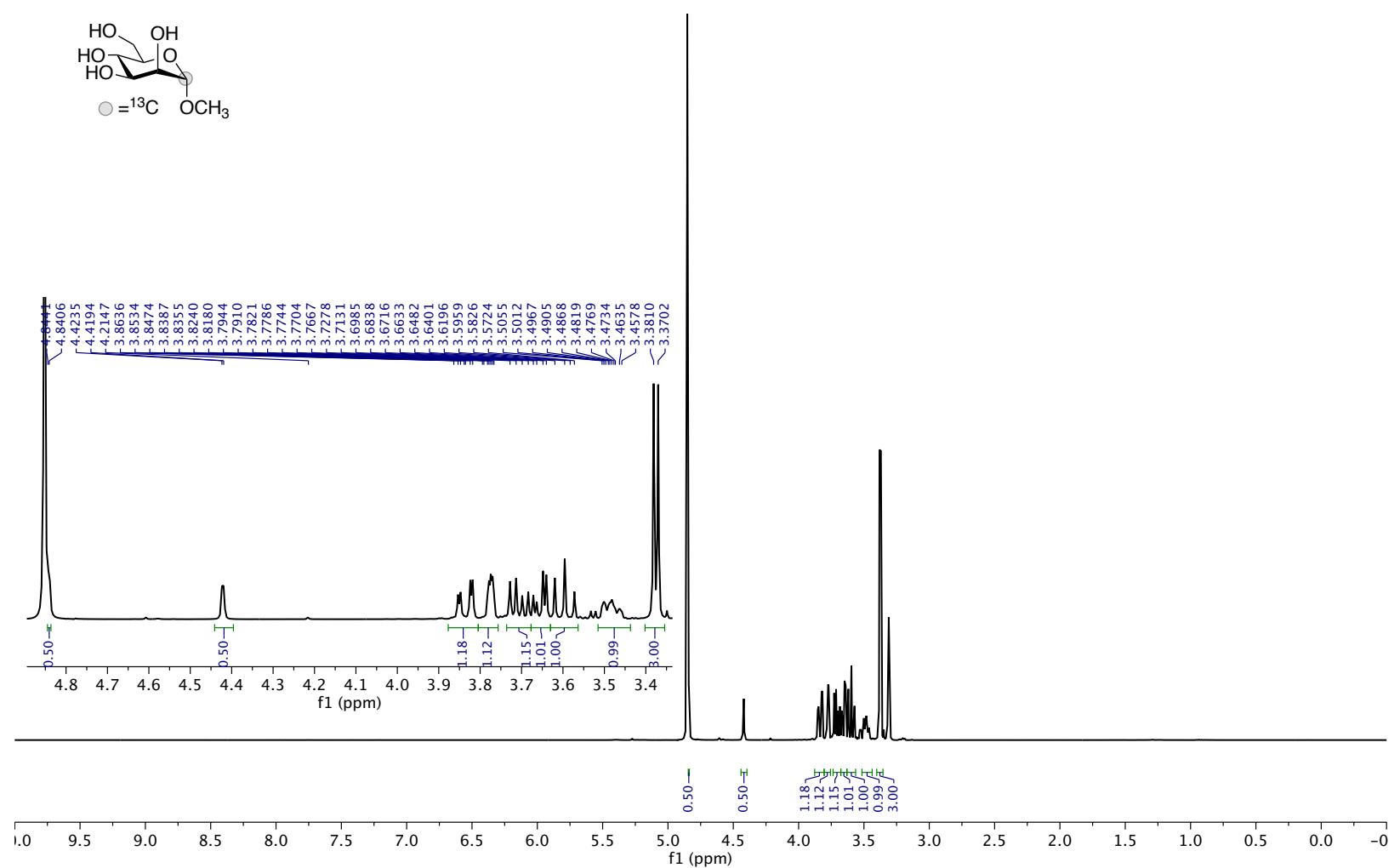

S10

${ }_{\mathrm{O}={ }^{13} \mathrm{C}}^{\mathrm{O} \mathrm{OCH}_{3}}$

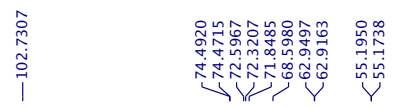

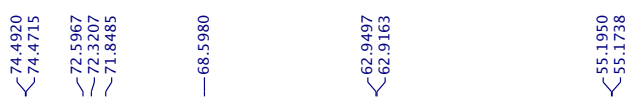

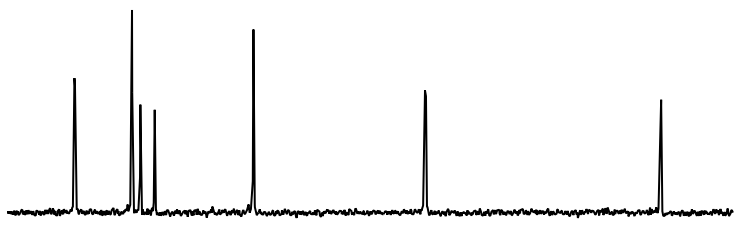

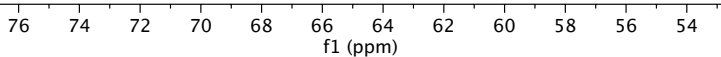

\begin{tabular}{lllllllllllllllllllllll}
\hline 10 & 200 & 190 & 180 & 170 & 160 & 150 & 140 & 130 & 120 & 110 & $\begin{array}{c}100 \\
\mathrm{f} 1(\mathrm{ppm})\end{array}$ & 90 & 80 & 70 & 60 & 50 & 40 & 30 & 20 & 10 & 0 & -1
\end{tabular} 


\section{S11}
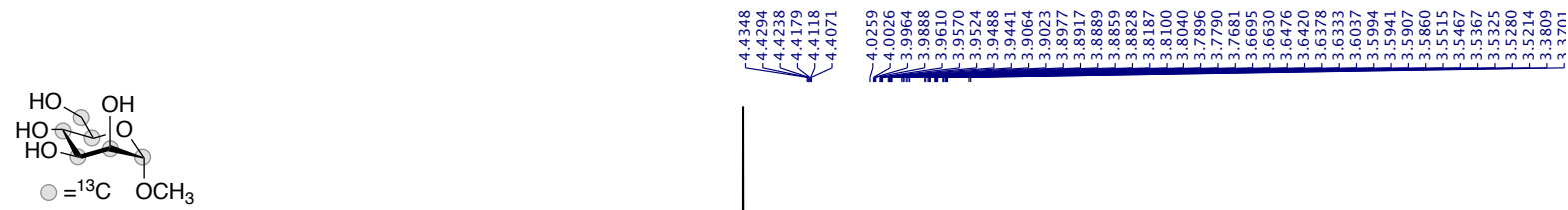

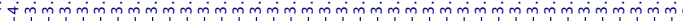

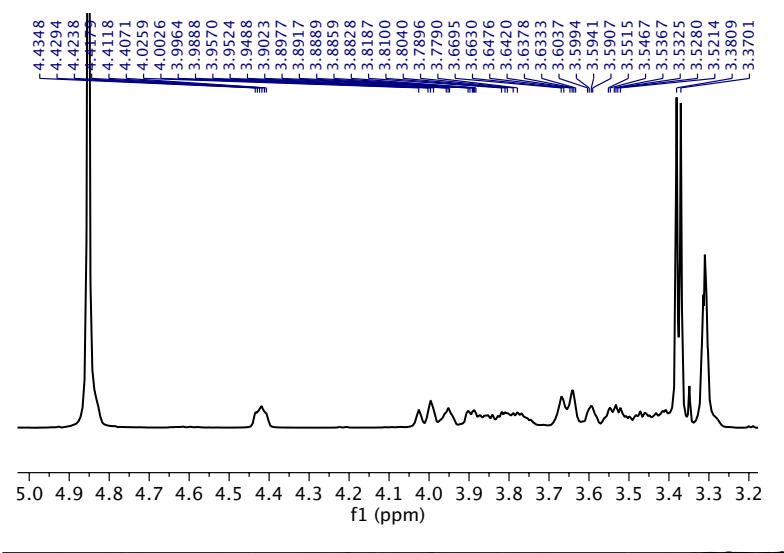

1 Mand han

1.0

5.0
$\mathrm{f} 1(\mathrm{ppm})$

S11

${ }_{={ }^{13} \mathrm{C} \quad \mathrm{OCH}_{3}}^{\mathrm{HO}}$

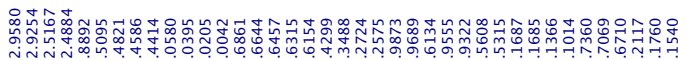

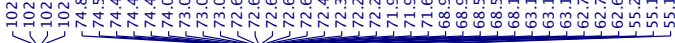

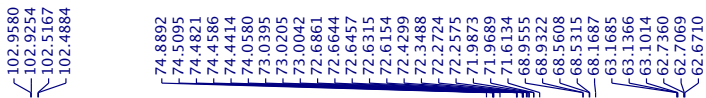
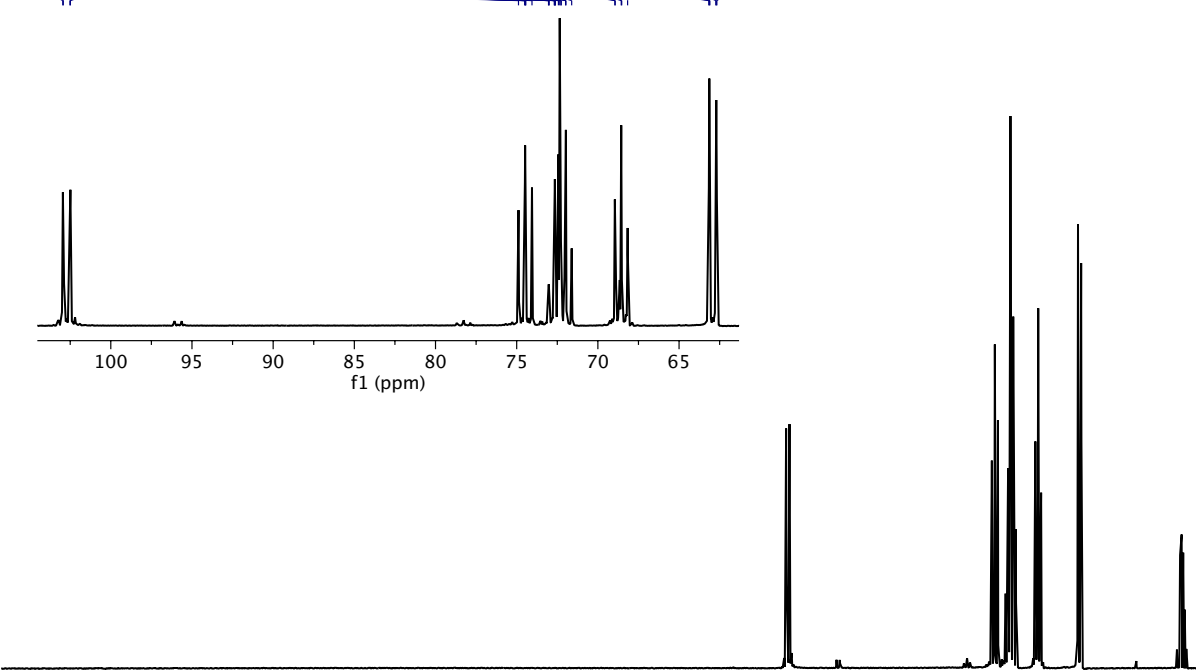

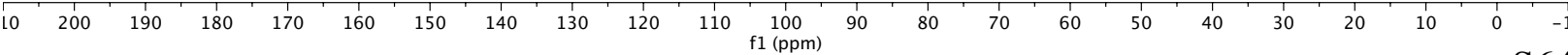




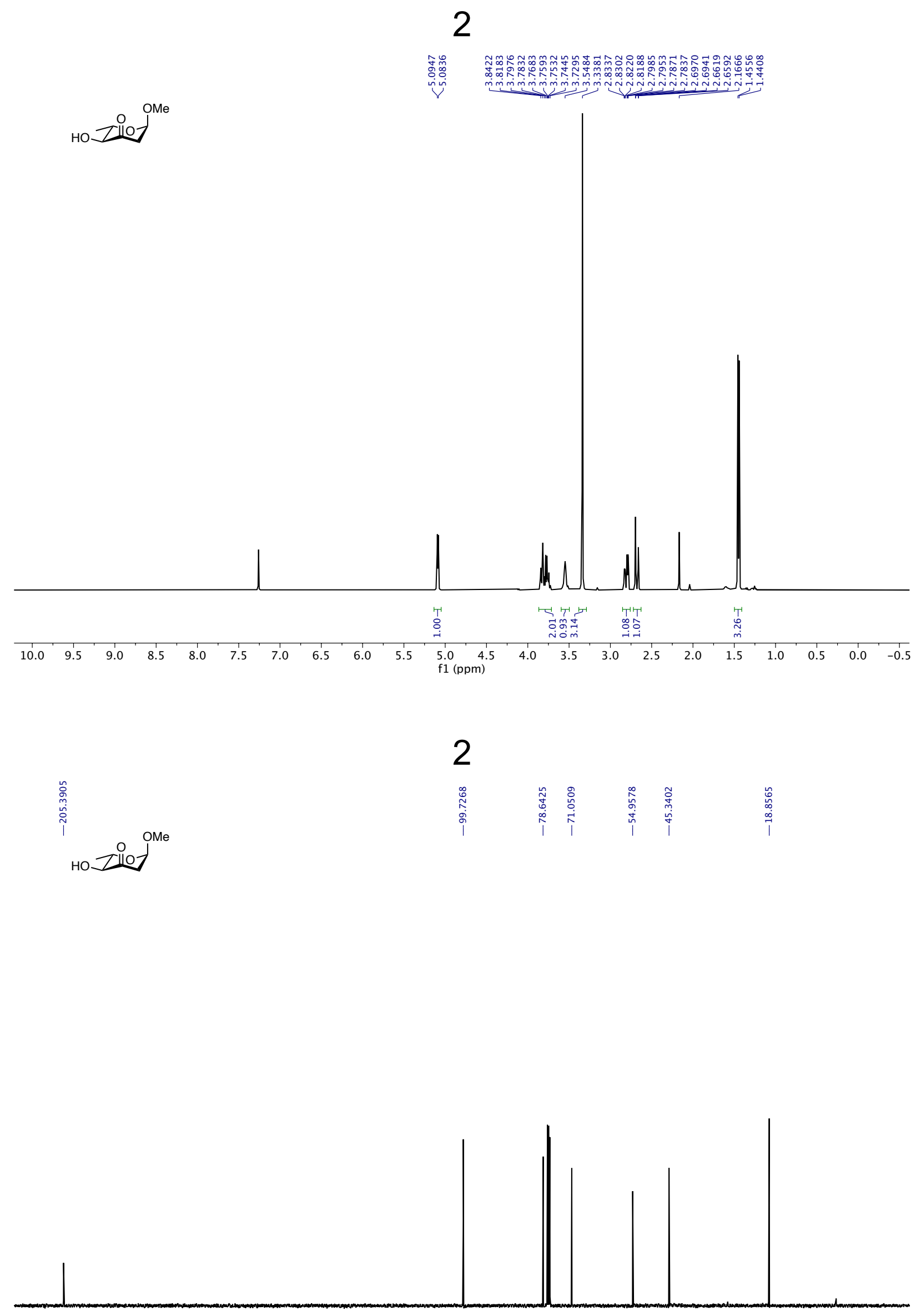

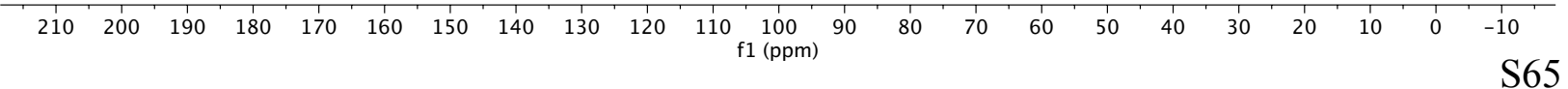


Ho tilloj

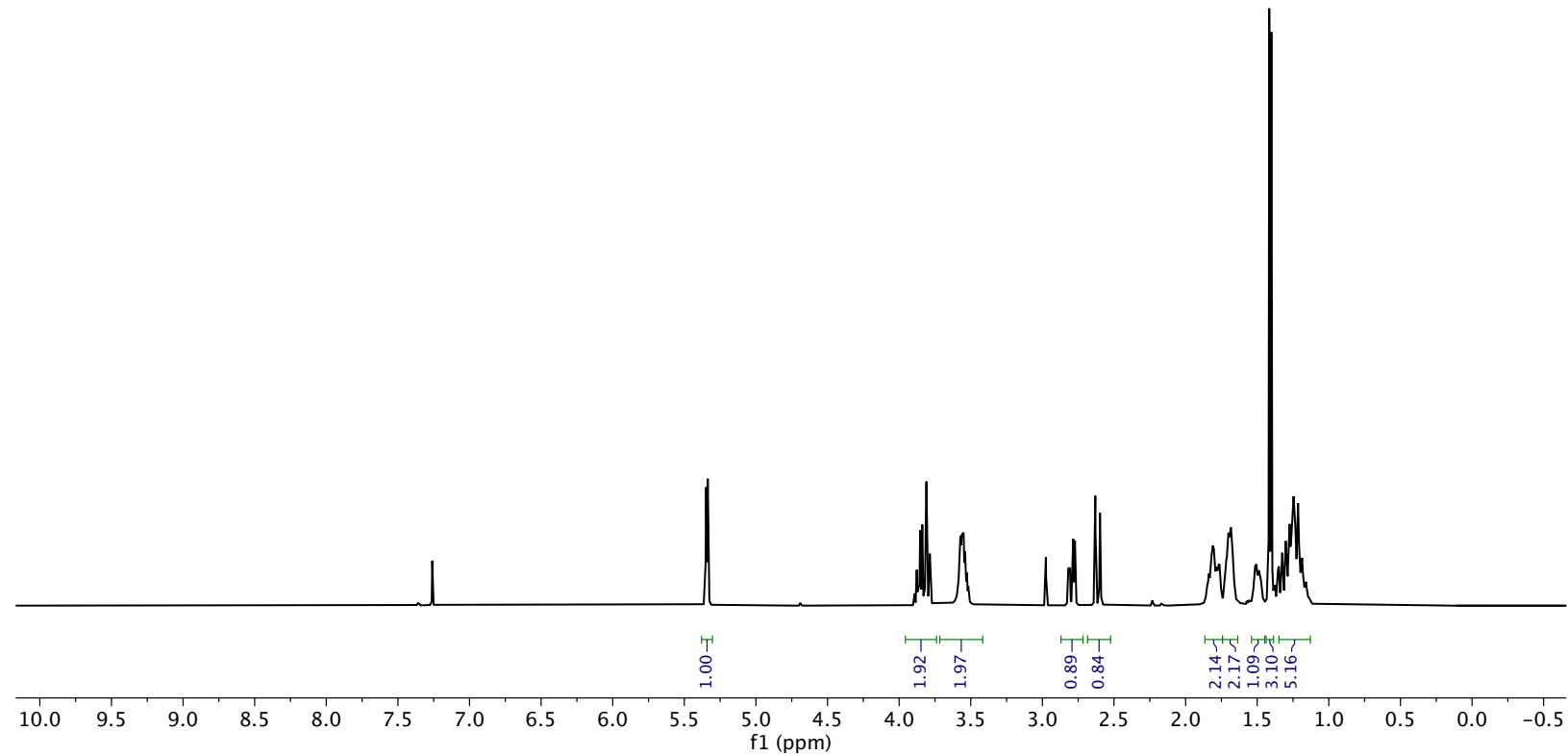

4

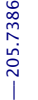

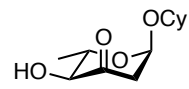

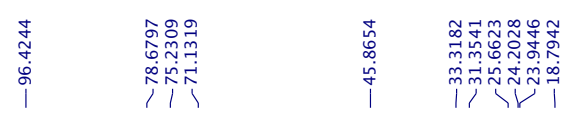

|||||||||||| $\mid$

$\begin{array}{rlllllllllllllllllllllllll}210 & 200 & 190 & 180 & 170 & 160 & 150 & 140 & 130 & 120 & 110 & \begin{array}{c}100 \\ \mathrm{f} 1(\mathrm{pbm})\end{array} & 90 & 80 & 70 & 60 & 50 & 40 & 30 & 20 & 10 & 0 & -10 \\ \mathrm{~S} 66\end{array}$




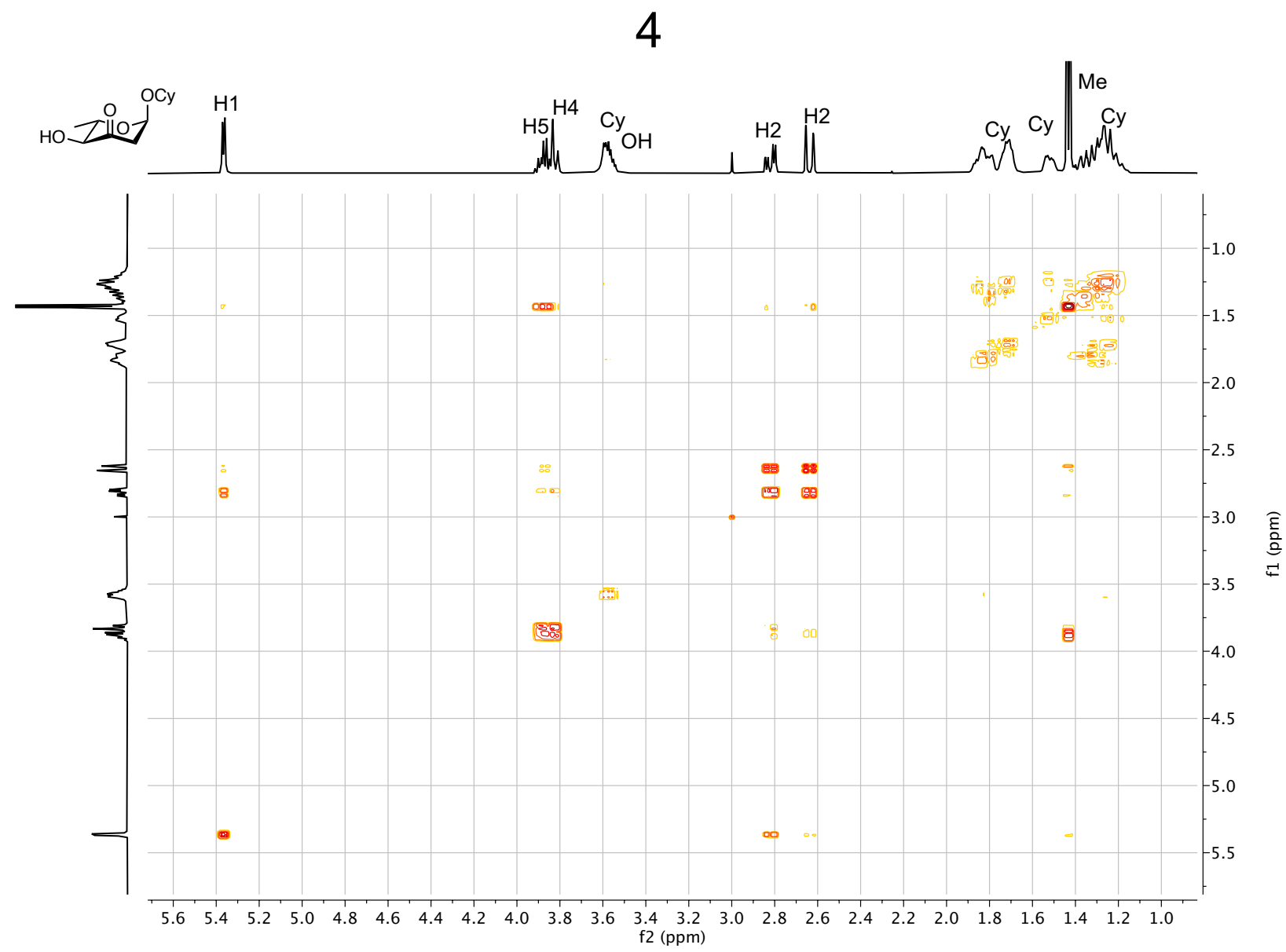

4

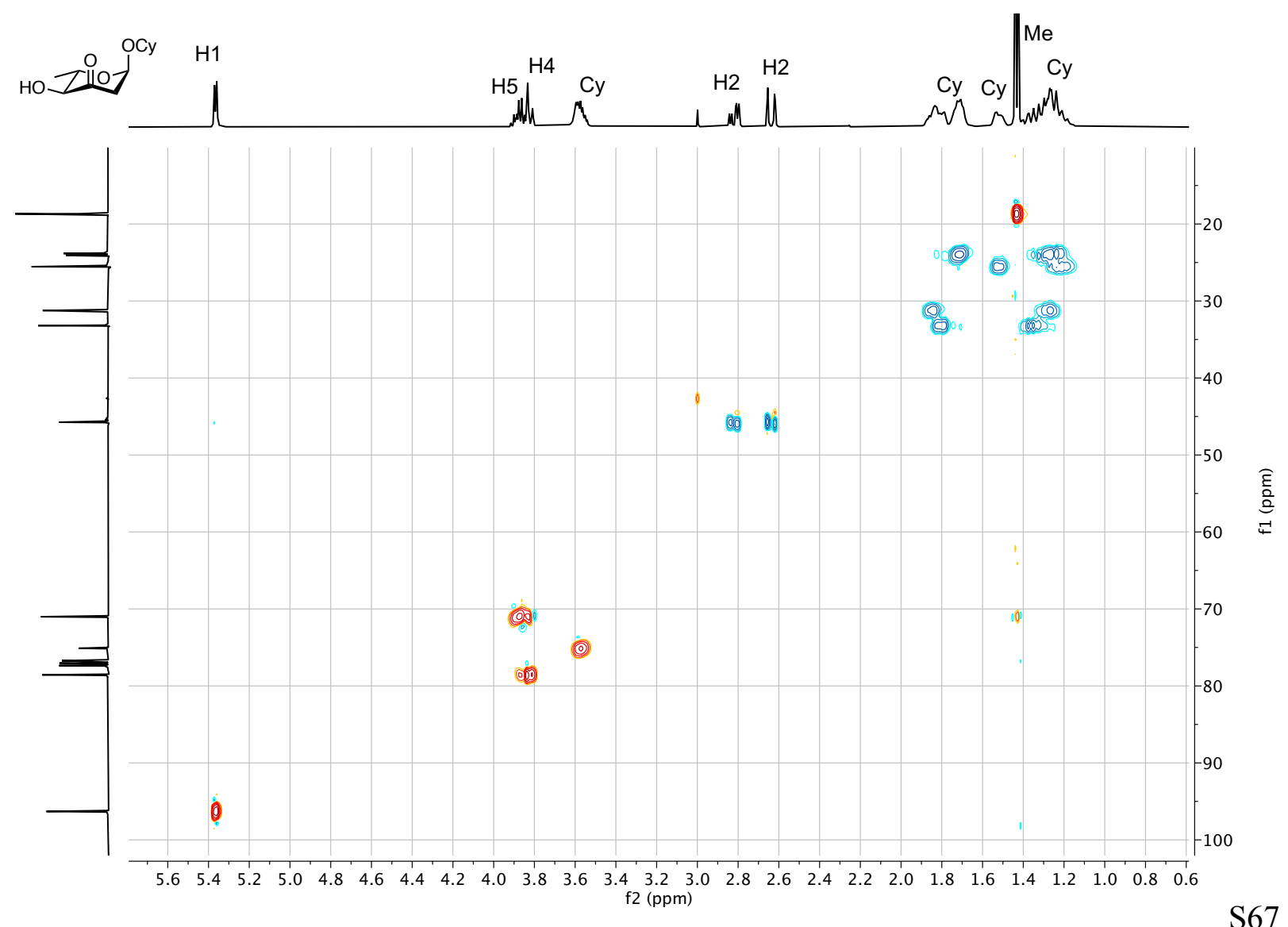




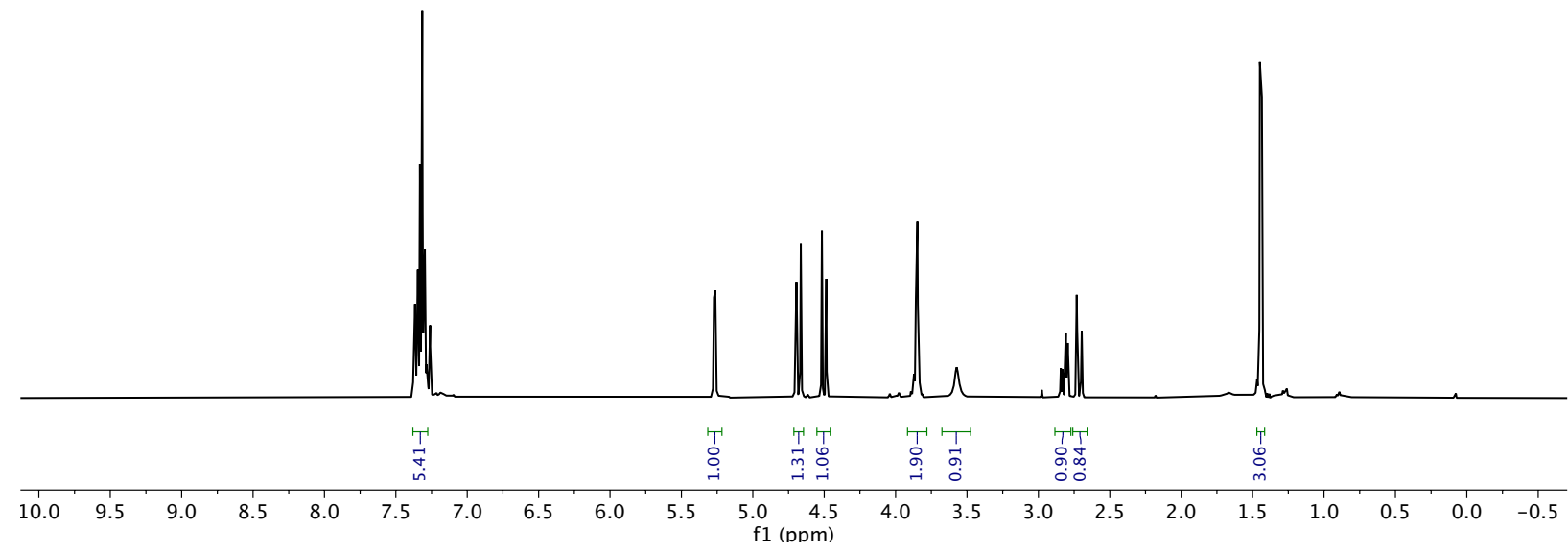

5

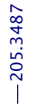
Ho ב-1이 
5

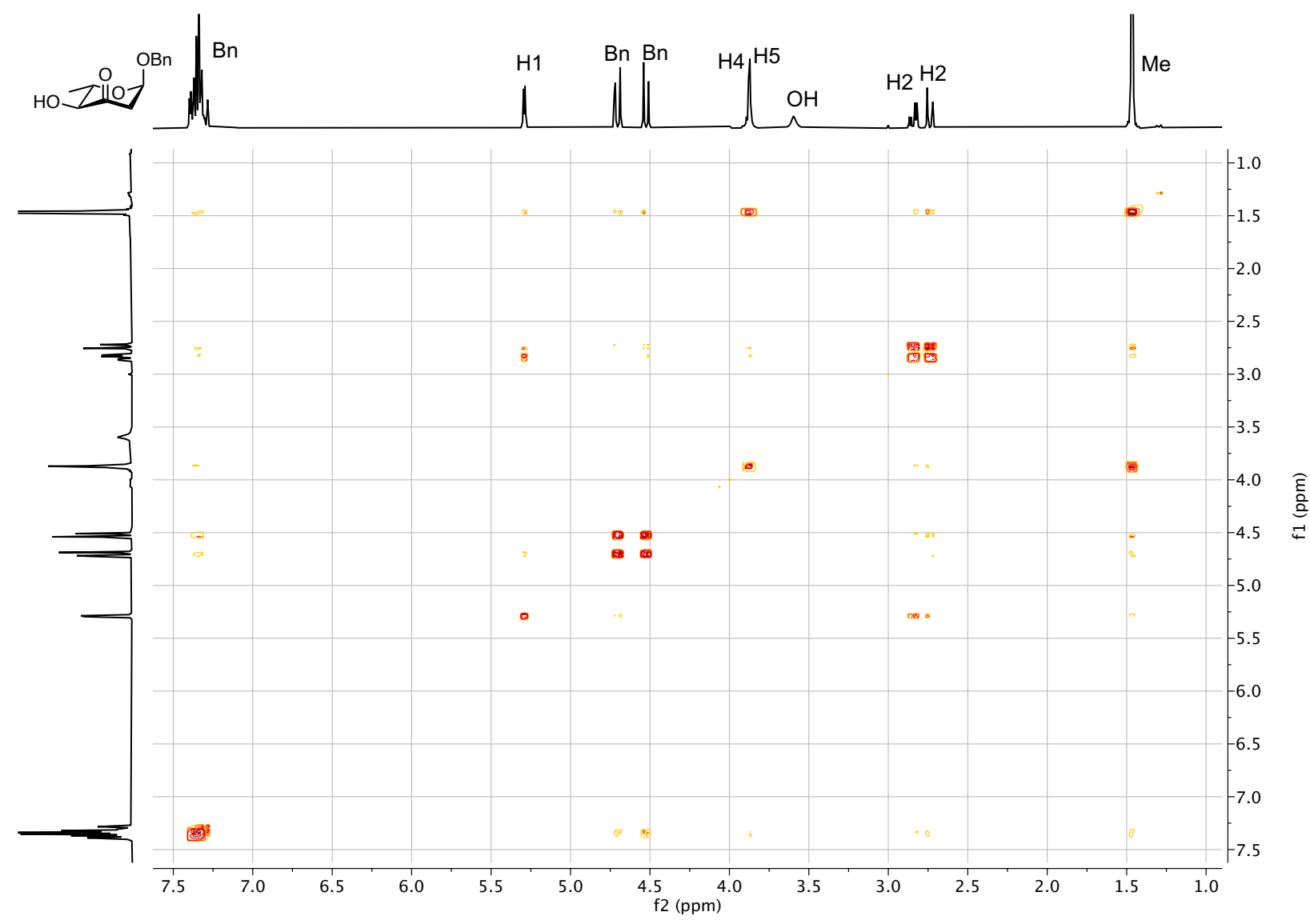

5
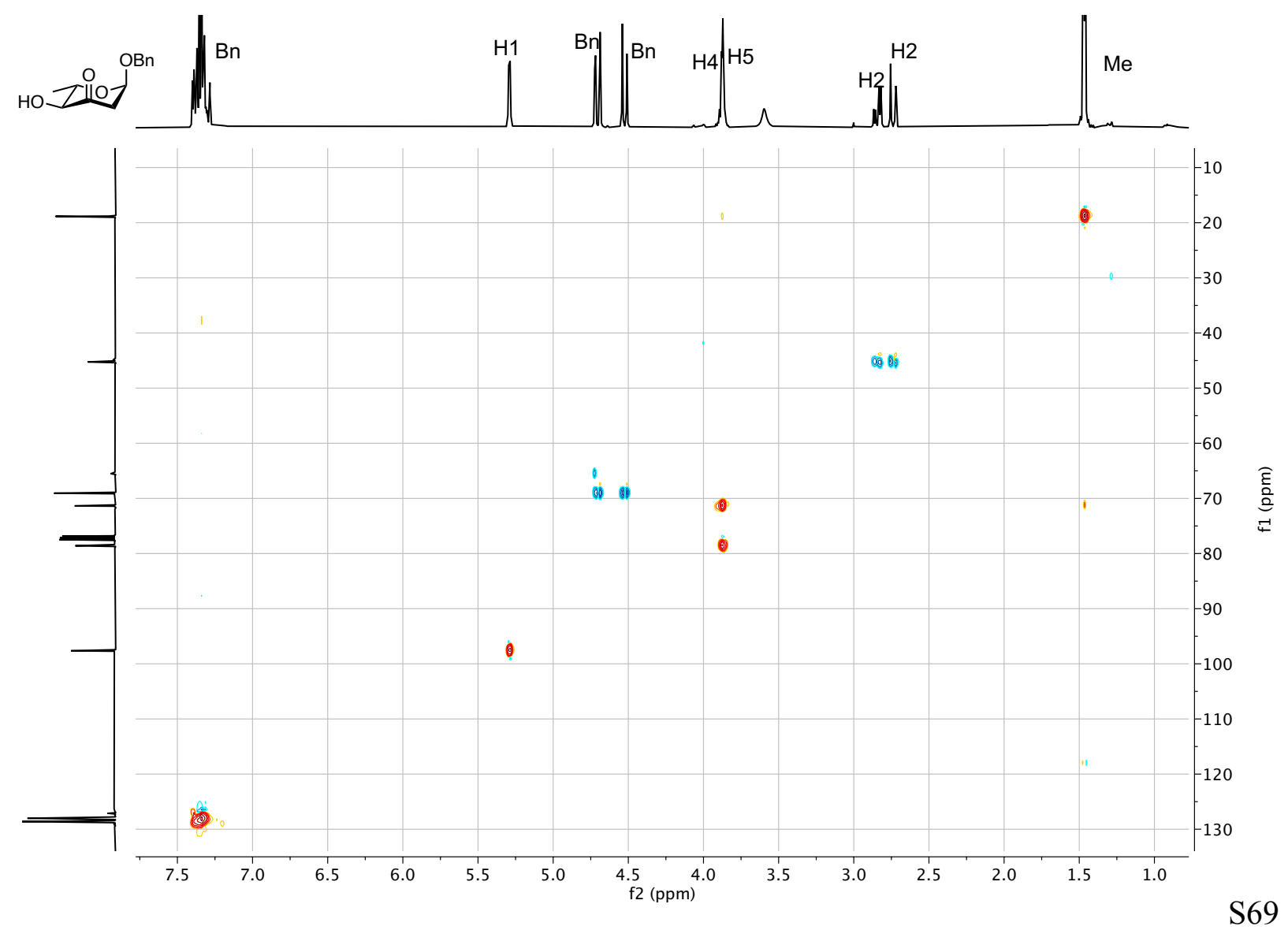


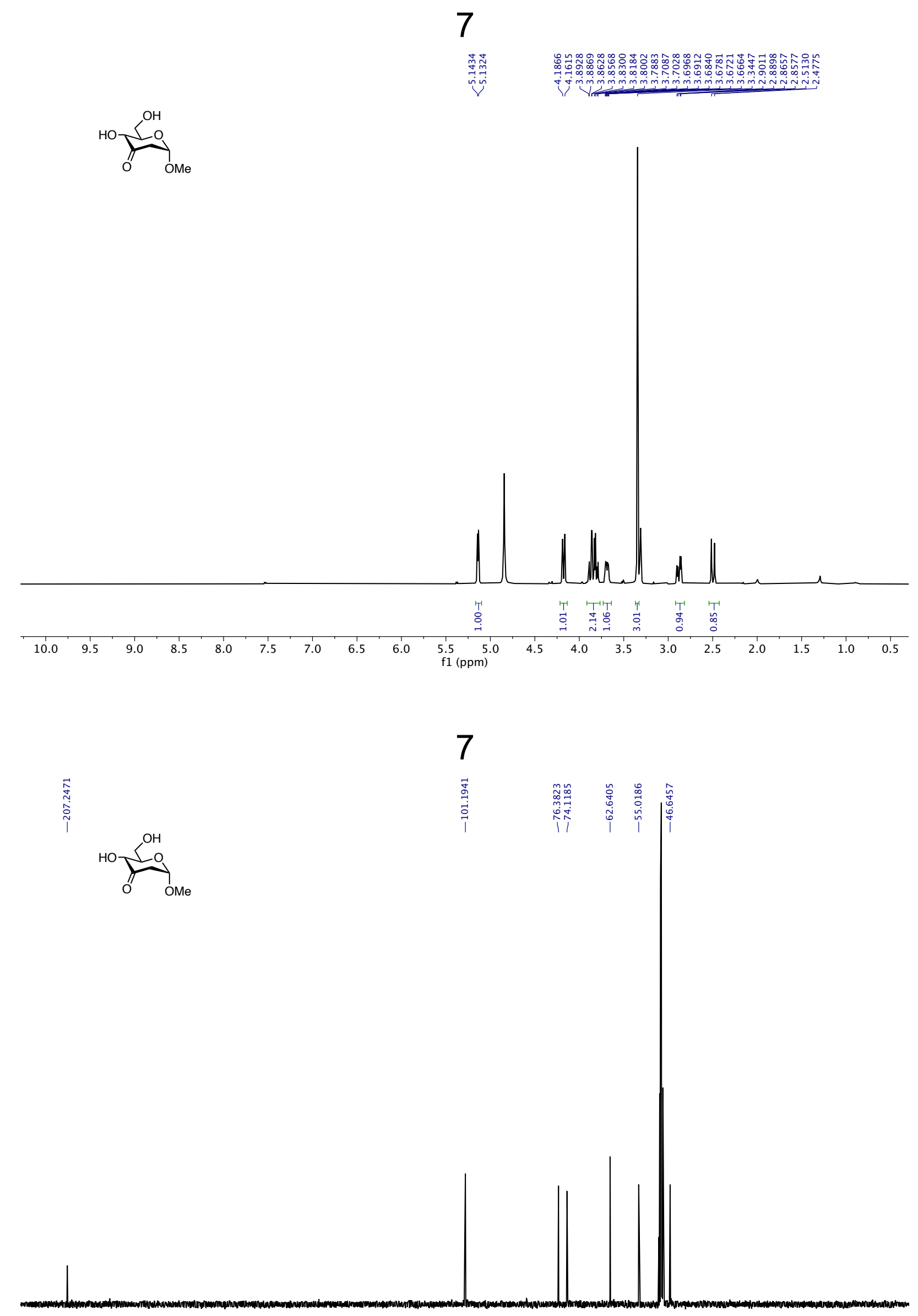

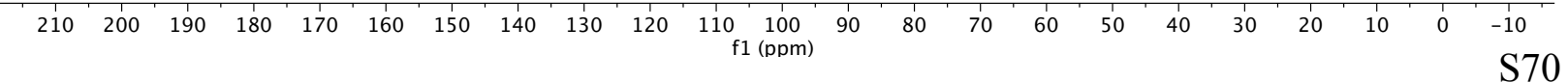



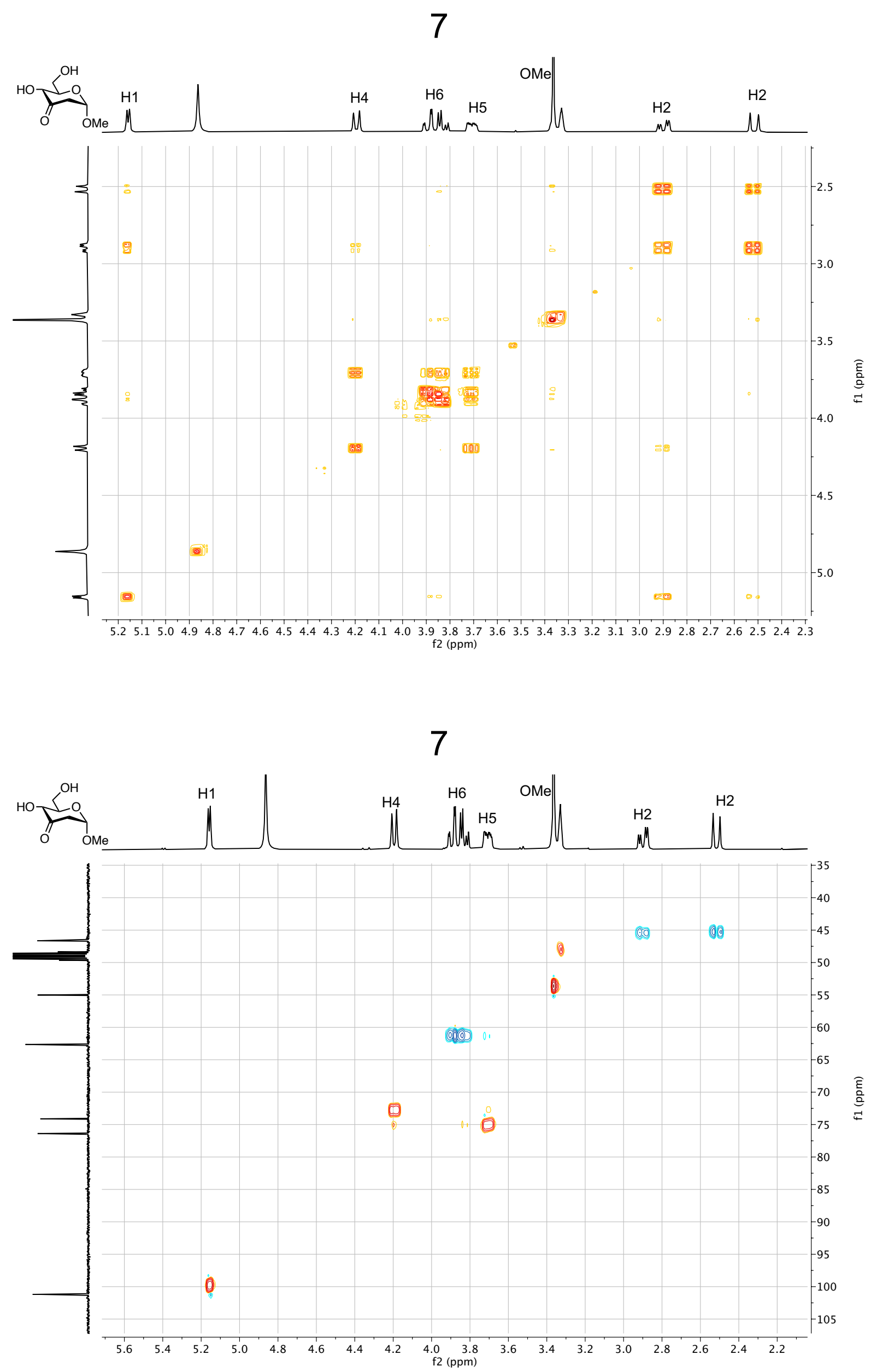


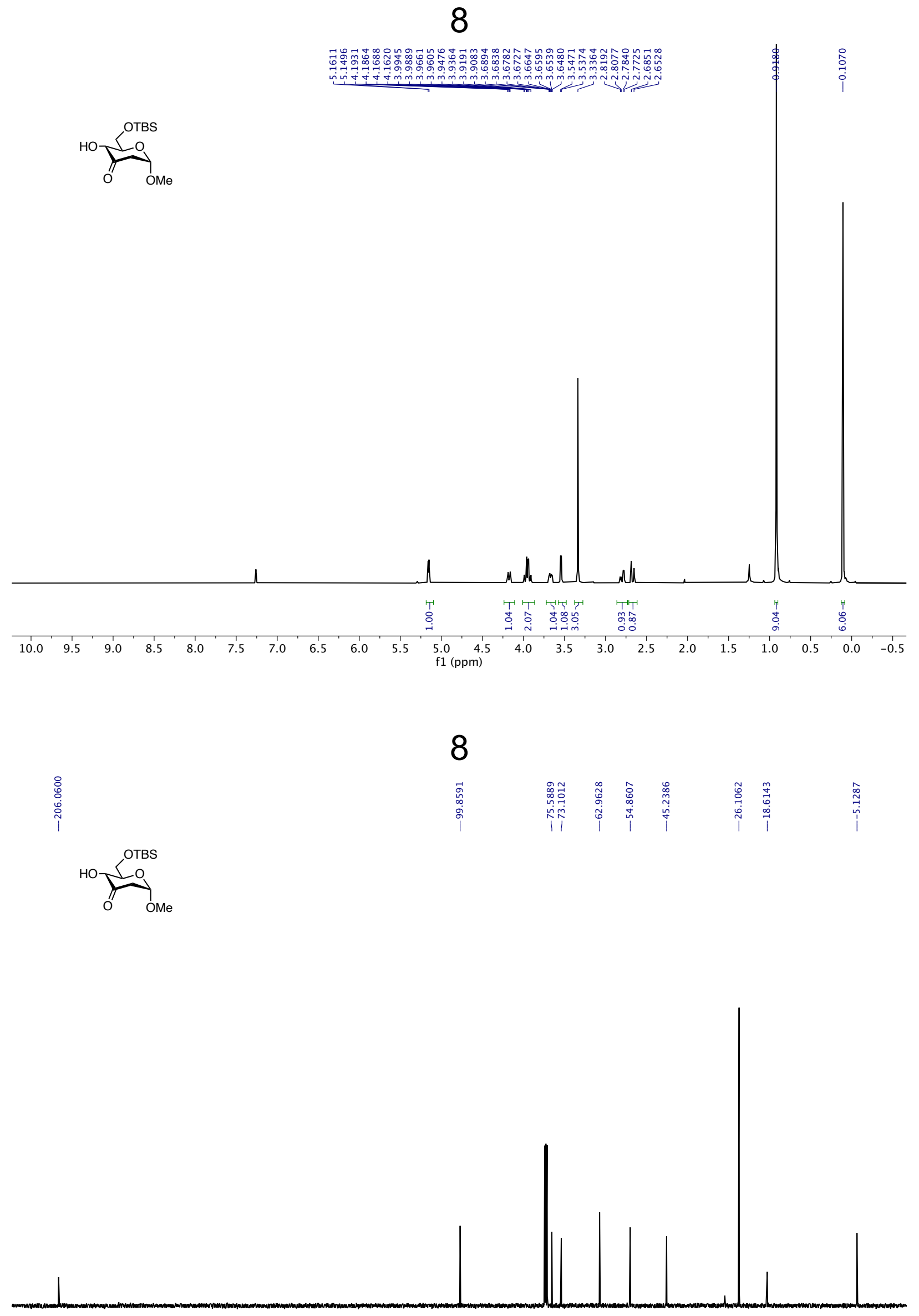

$\begin{array}{rllllllllllllllllllllll}210 & 200 & 190 & 180 & 170 & 160 & 150 & 140 & 130 & 120 & 110 & \begin{array}{c}100 \\ \mathrm{f} 1(\mathrm{ppm})\end{array} & 90 & 80 & 70 & 60 & 50 & 40 & 30 & 20 & 10 & 0 & -10 \\ \mathrm{~S} 72\end{array}$ 


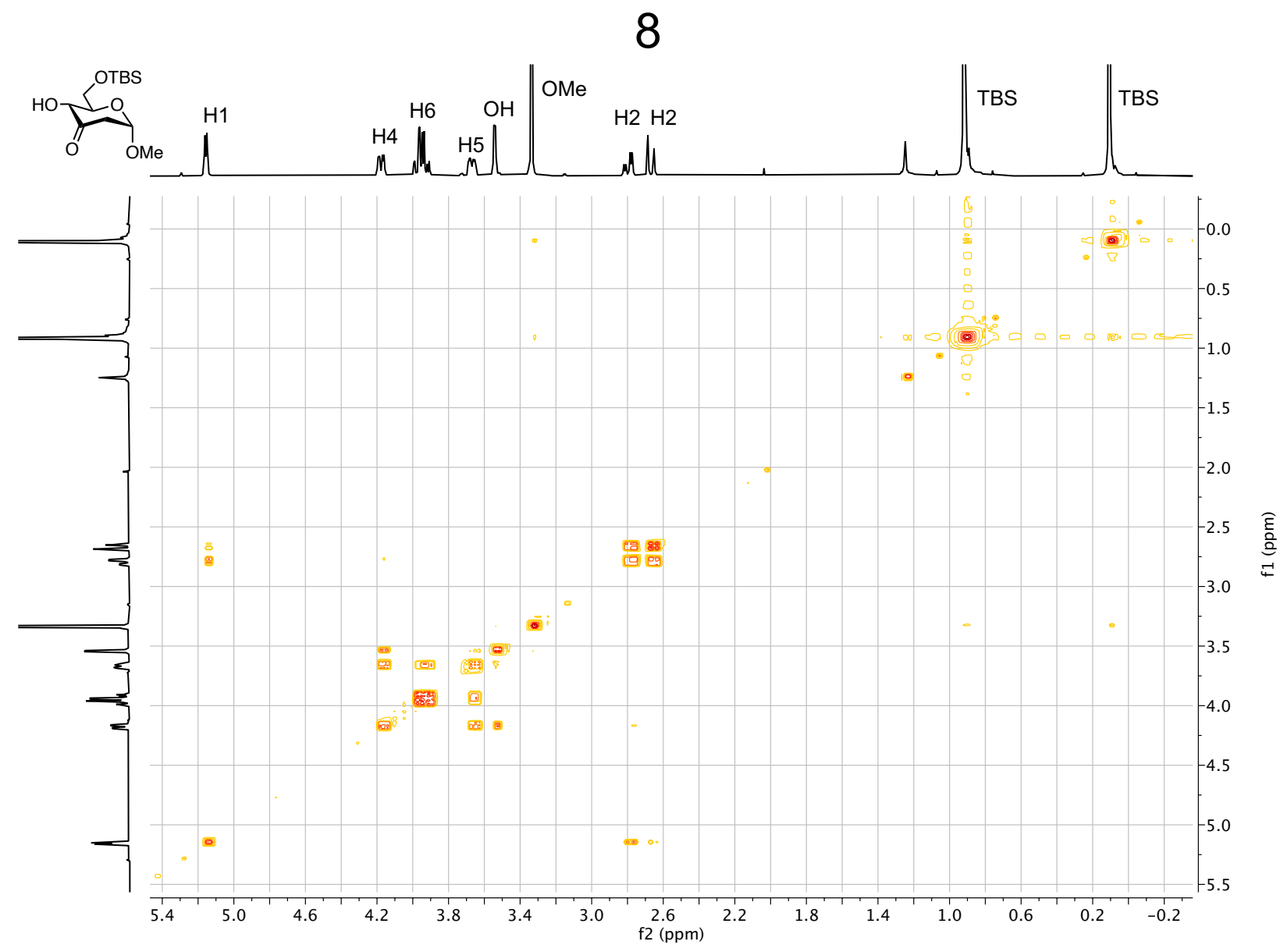

8

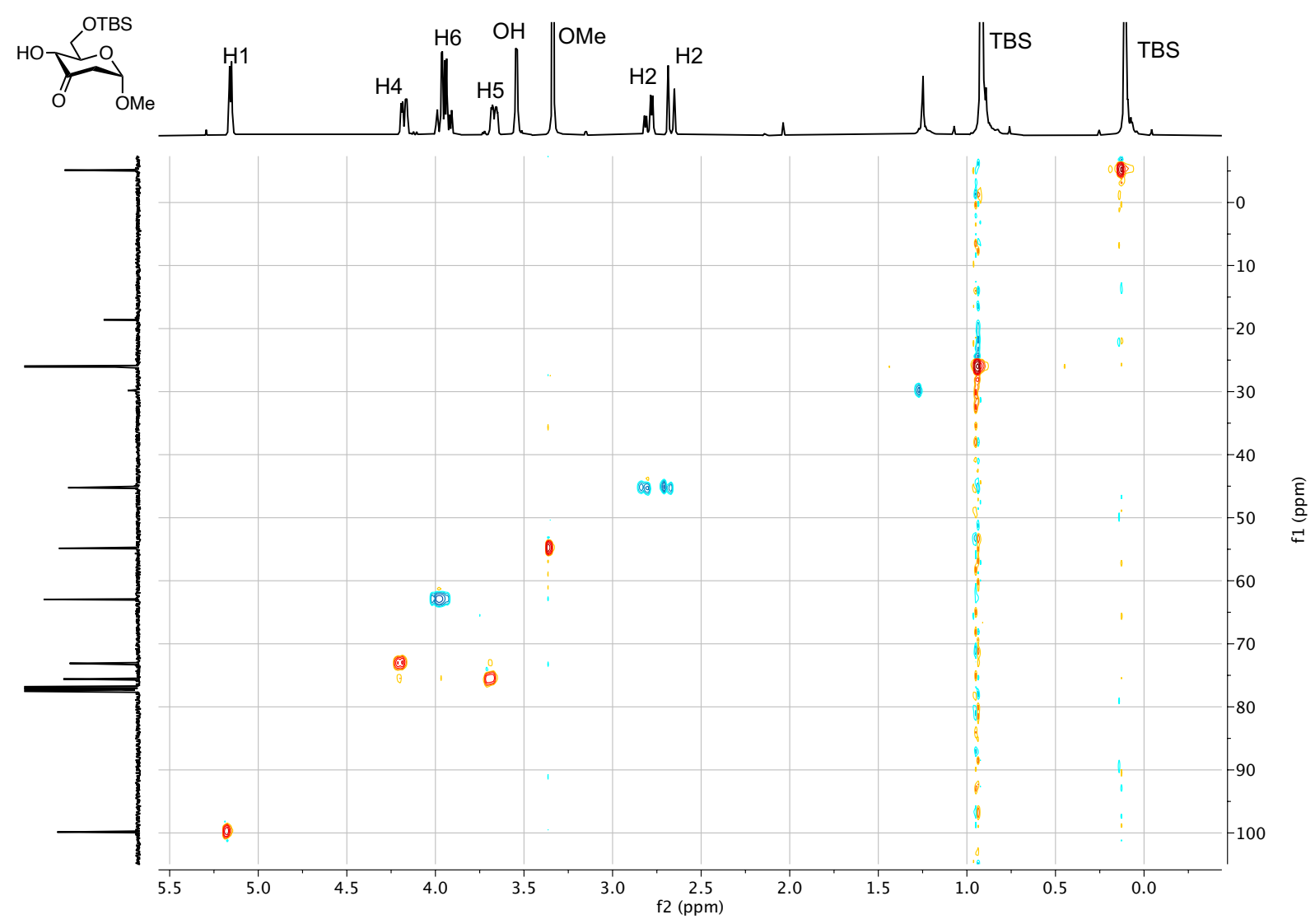


<smiles>O=C1[C+]2COC1C2</smiles>

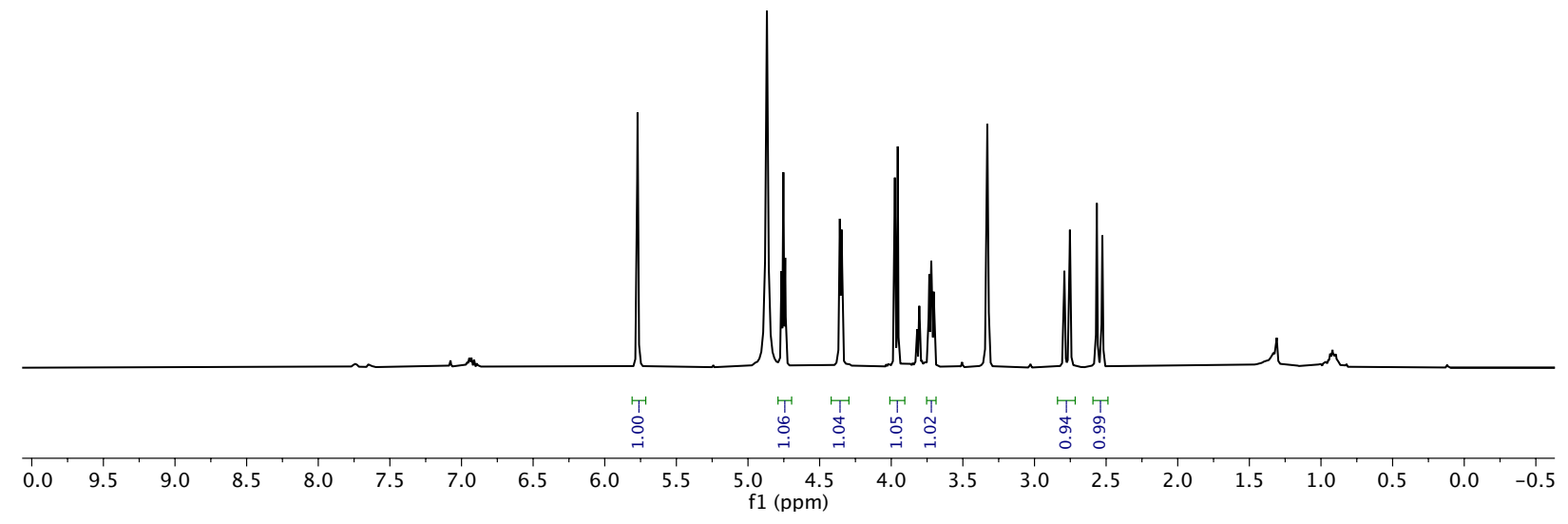

10

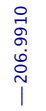

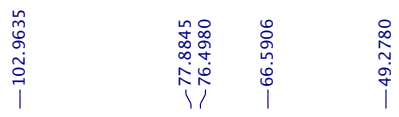<smiles>O=C1[C+]2COC1C2</smiles> 
10

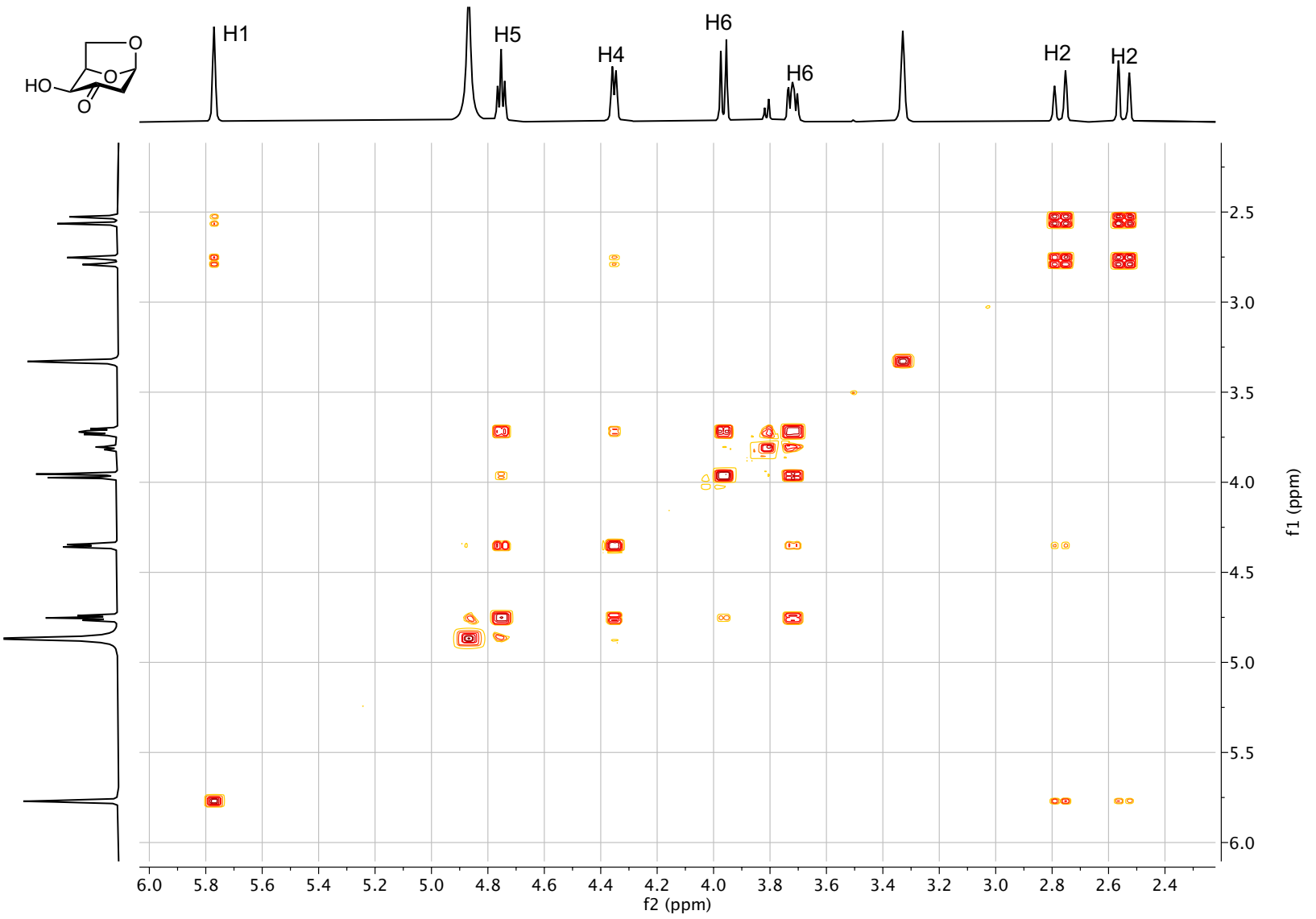

10

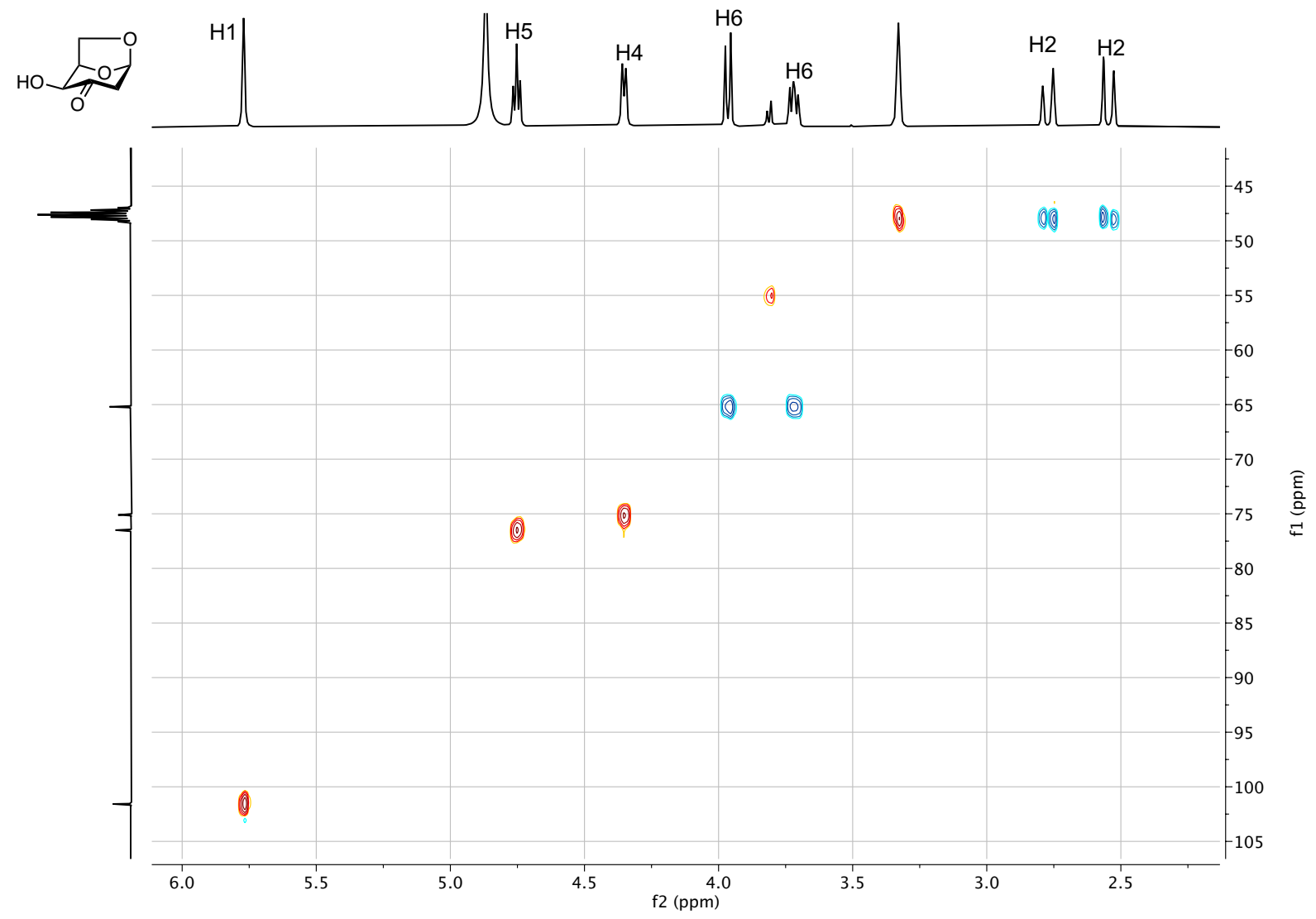



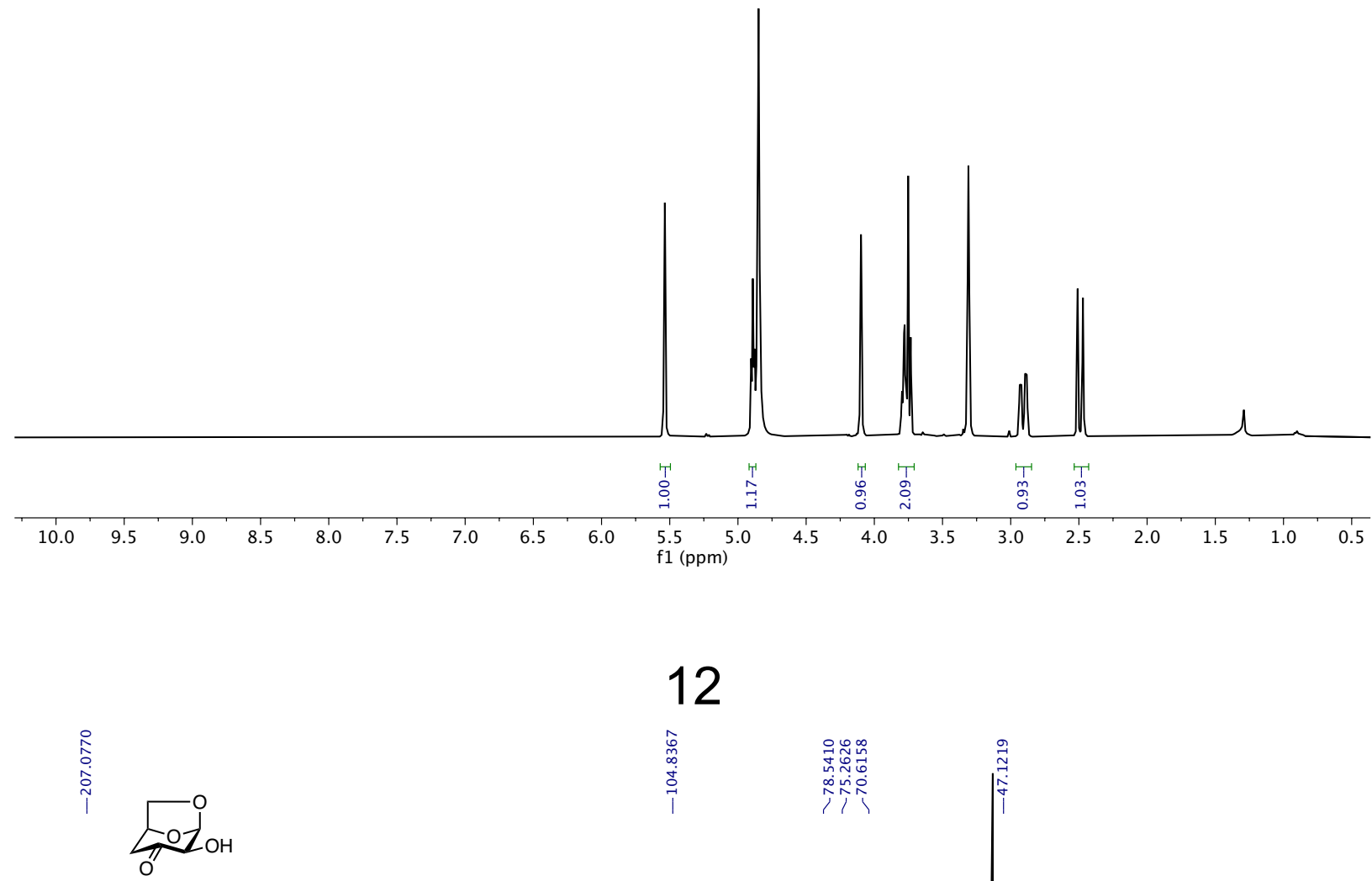

12
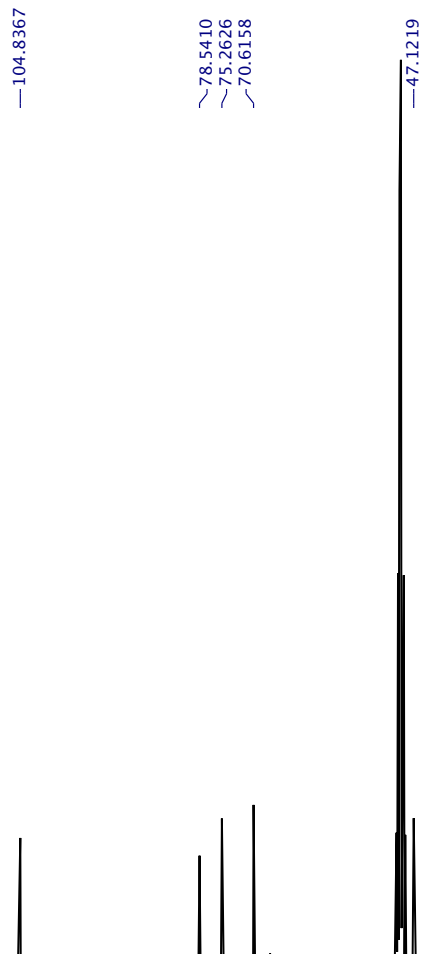
12

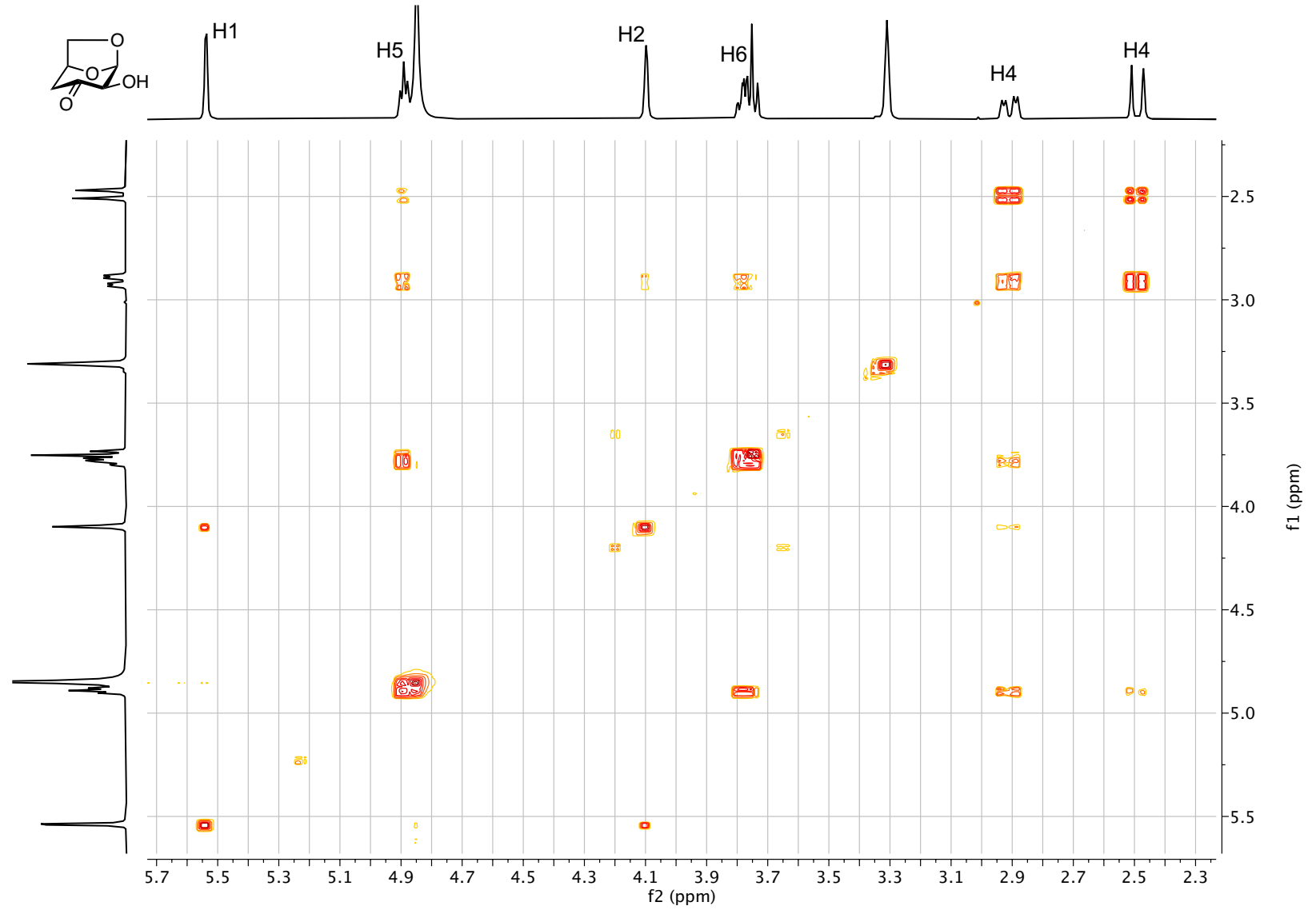

12

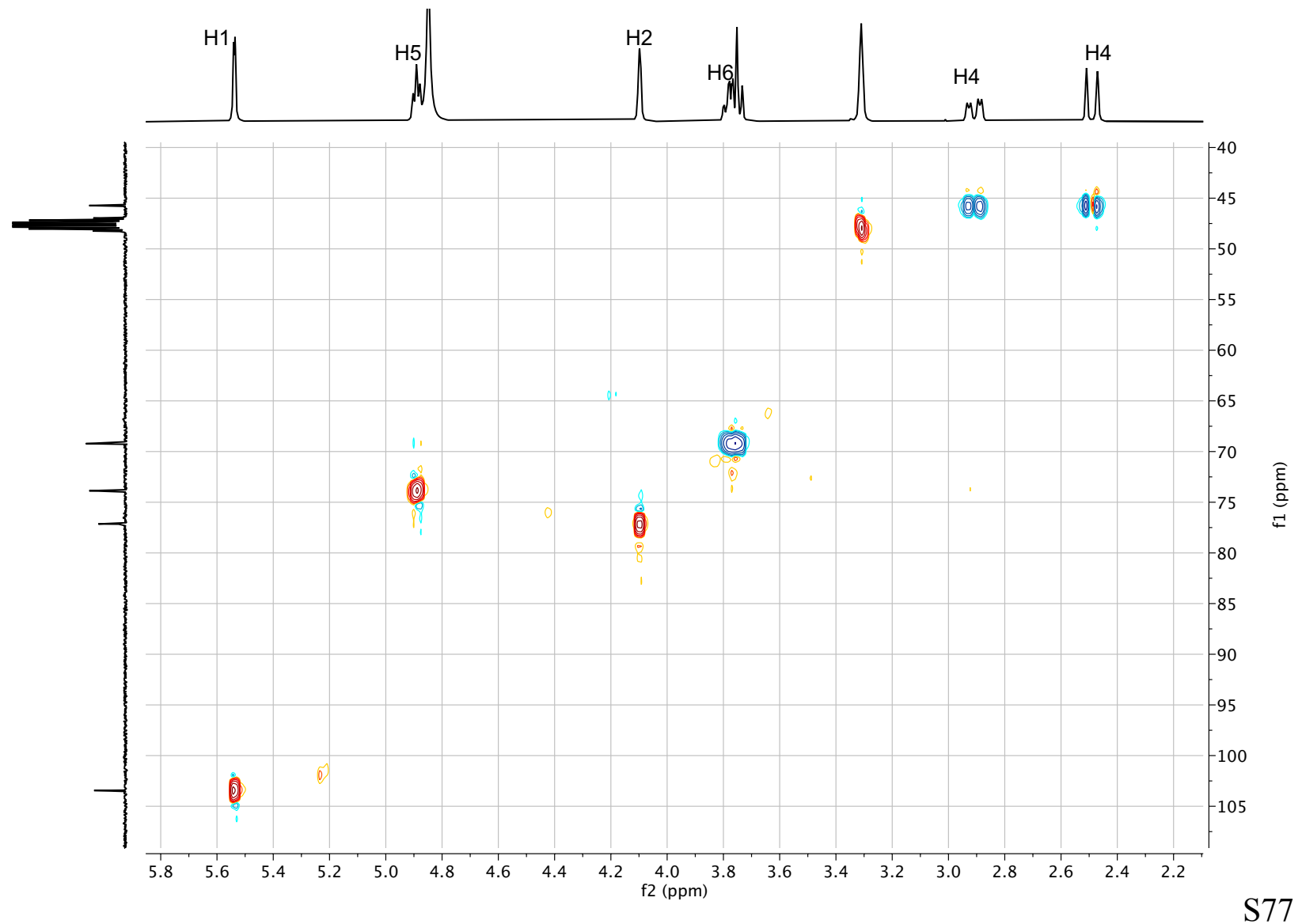




\section{5 and 16}
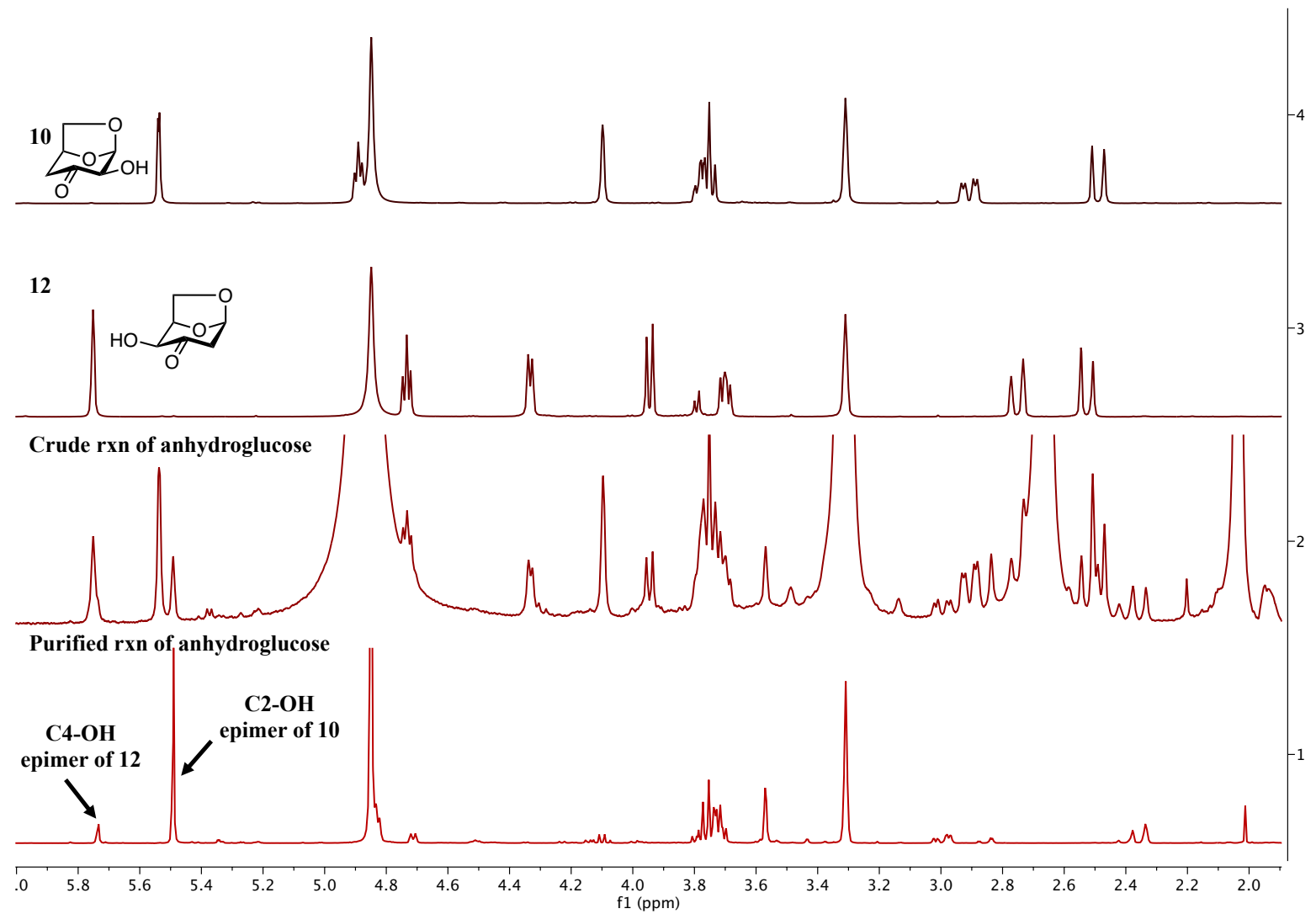

18
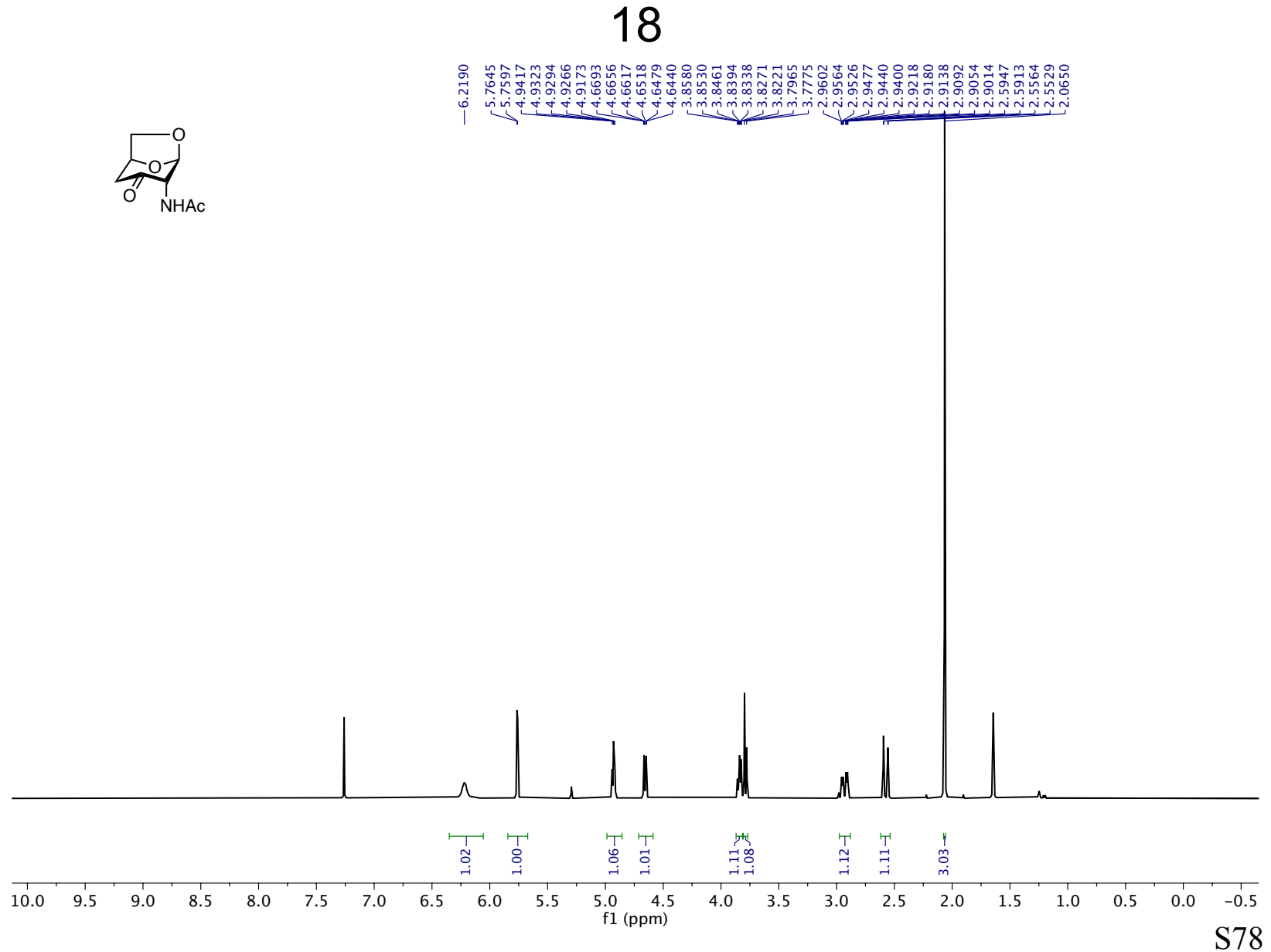
18

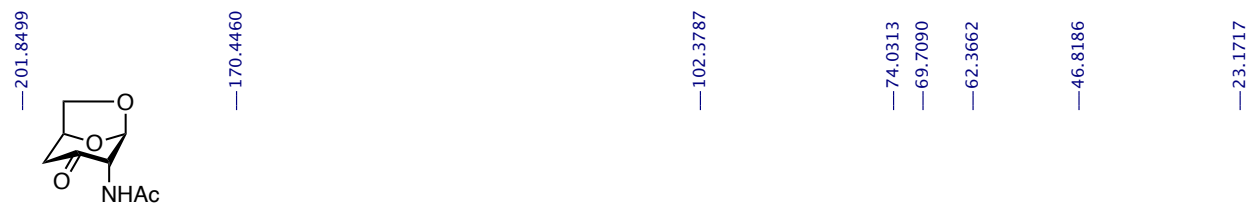
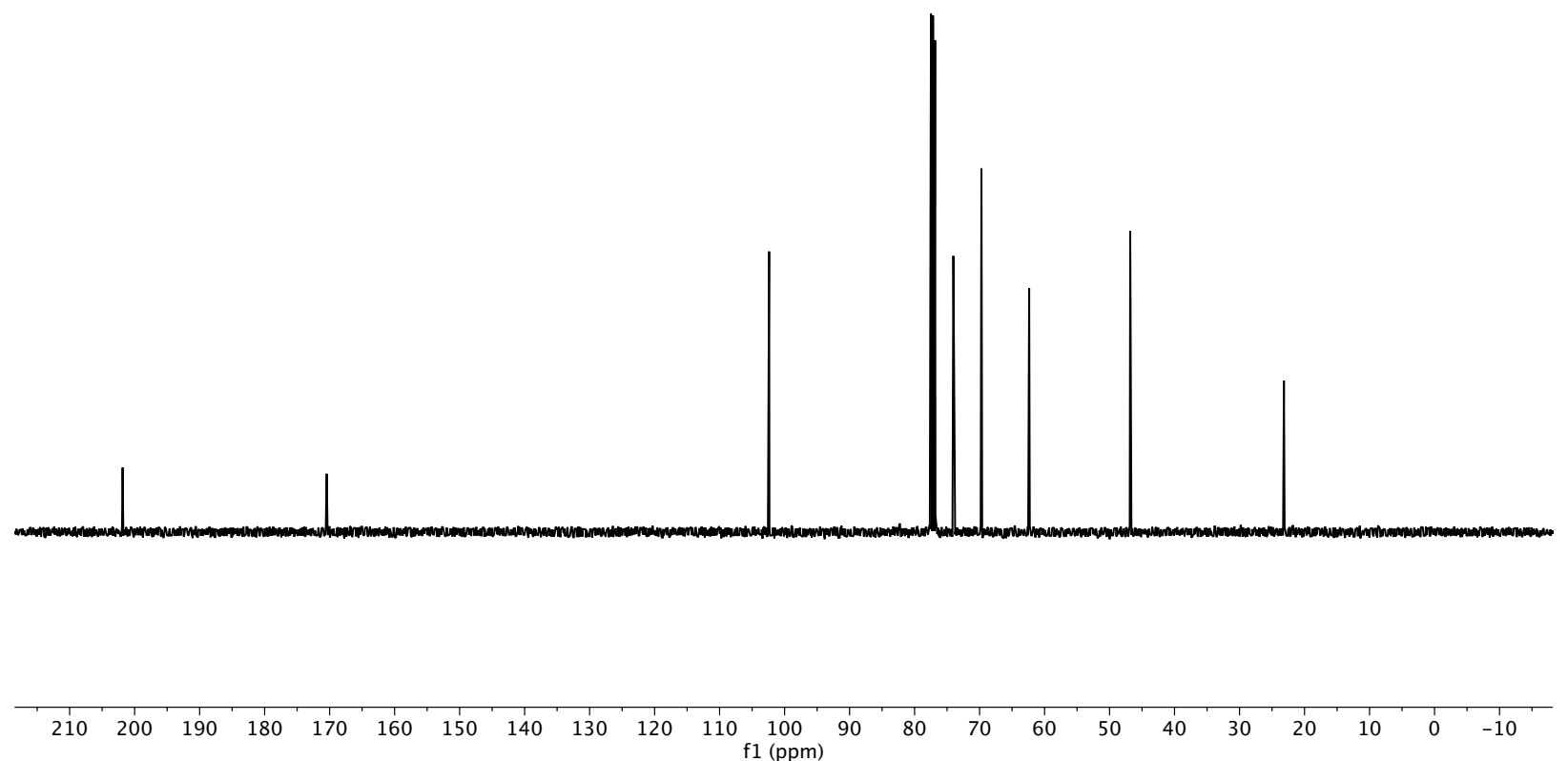

18

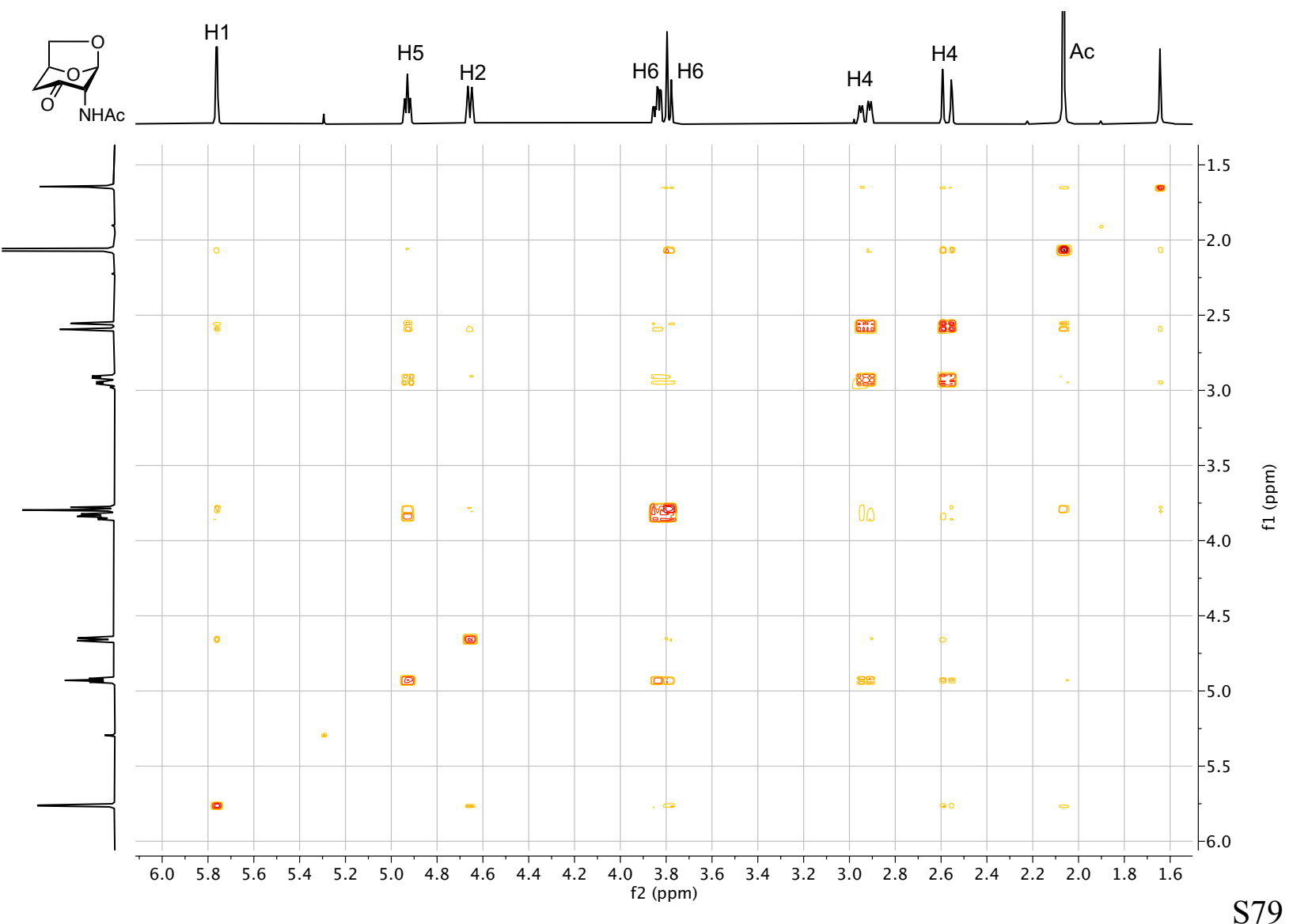


18

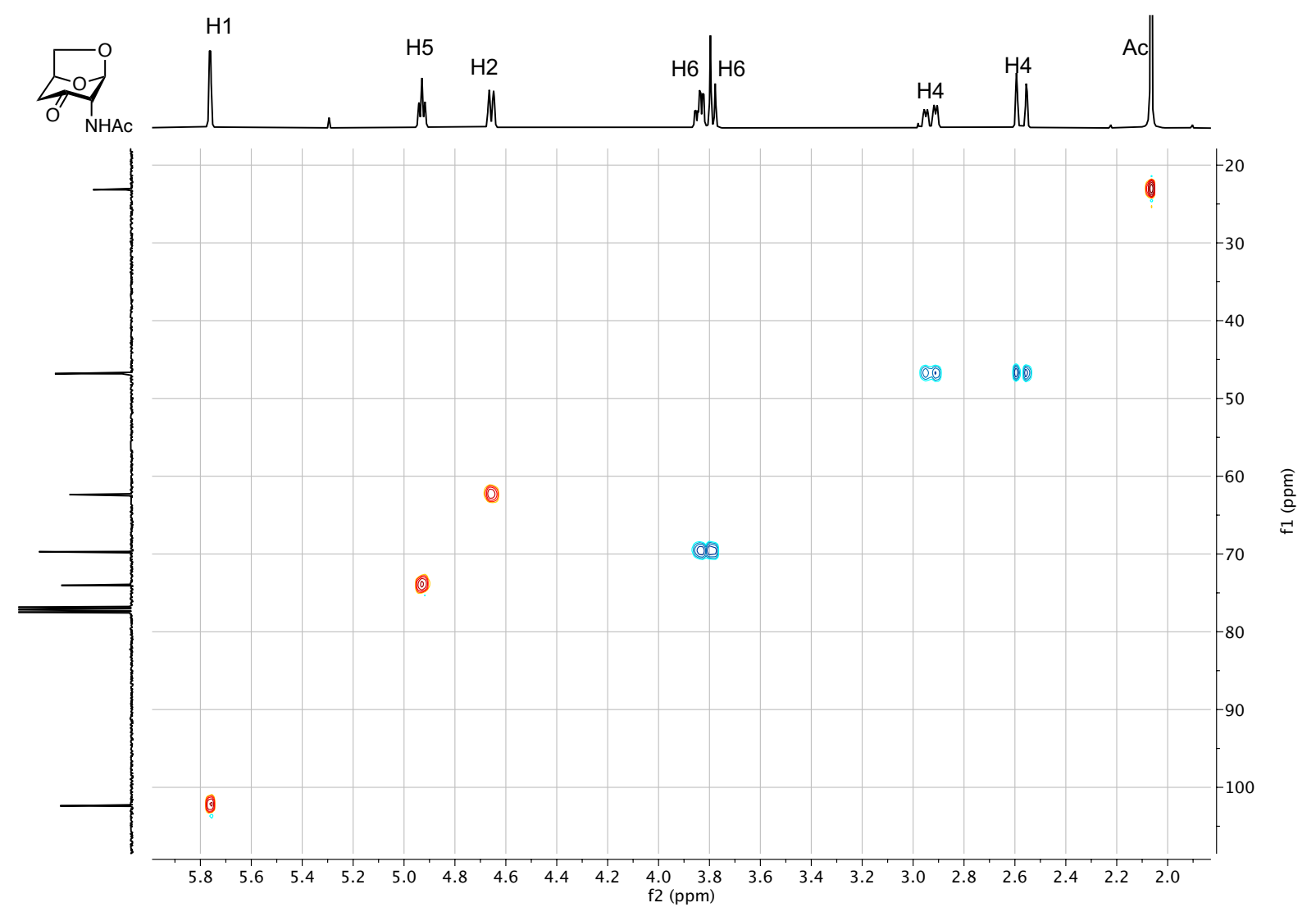

21

$\overbrace{\mathrm{O}}^{\mathrm{OH}}$

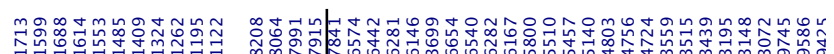

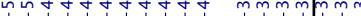

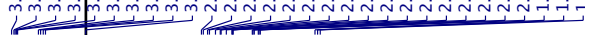

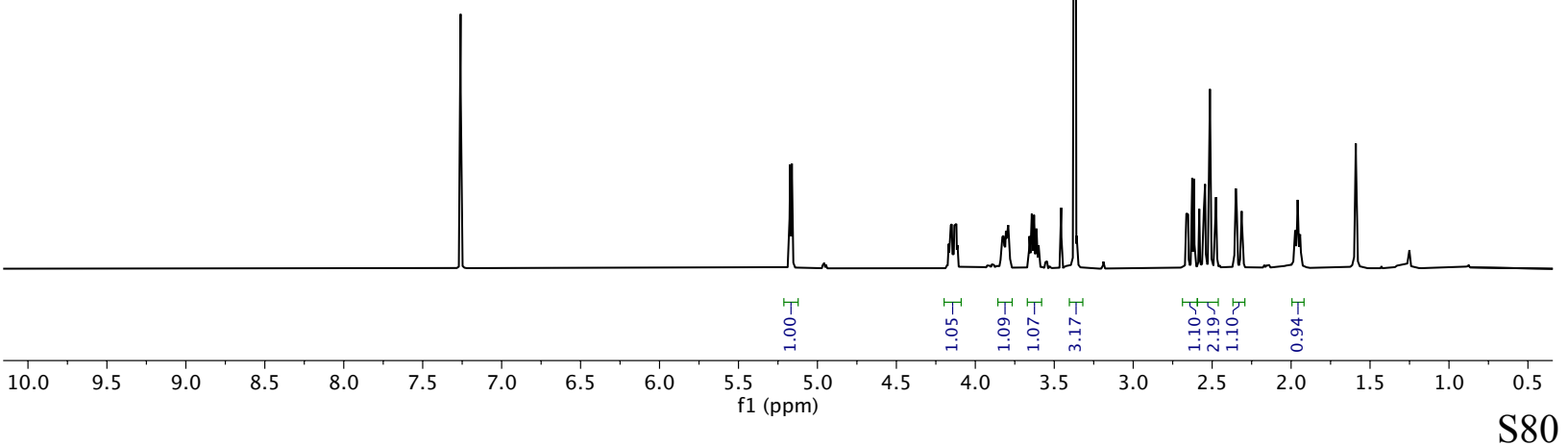


21

i

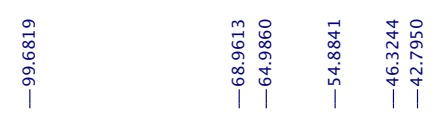

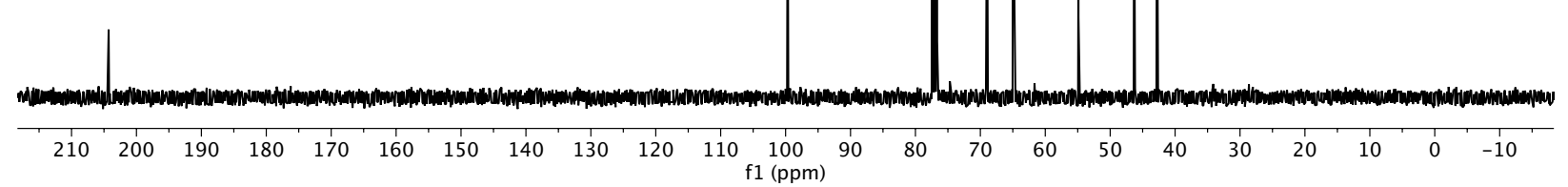

21

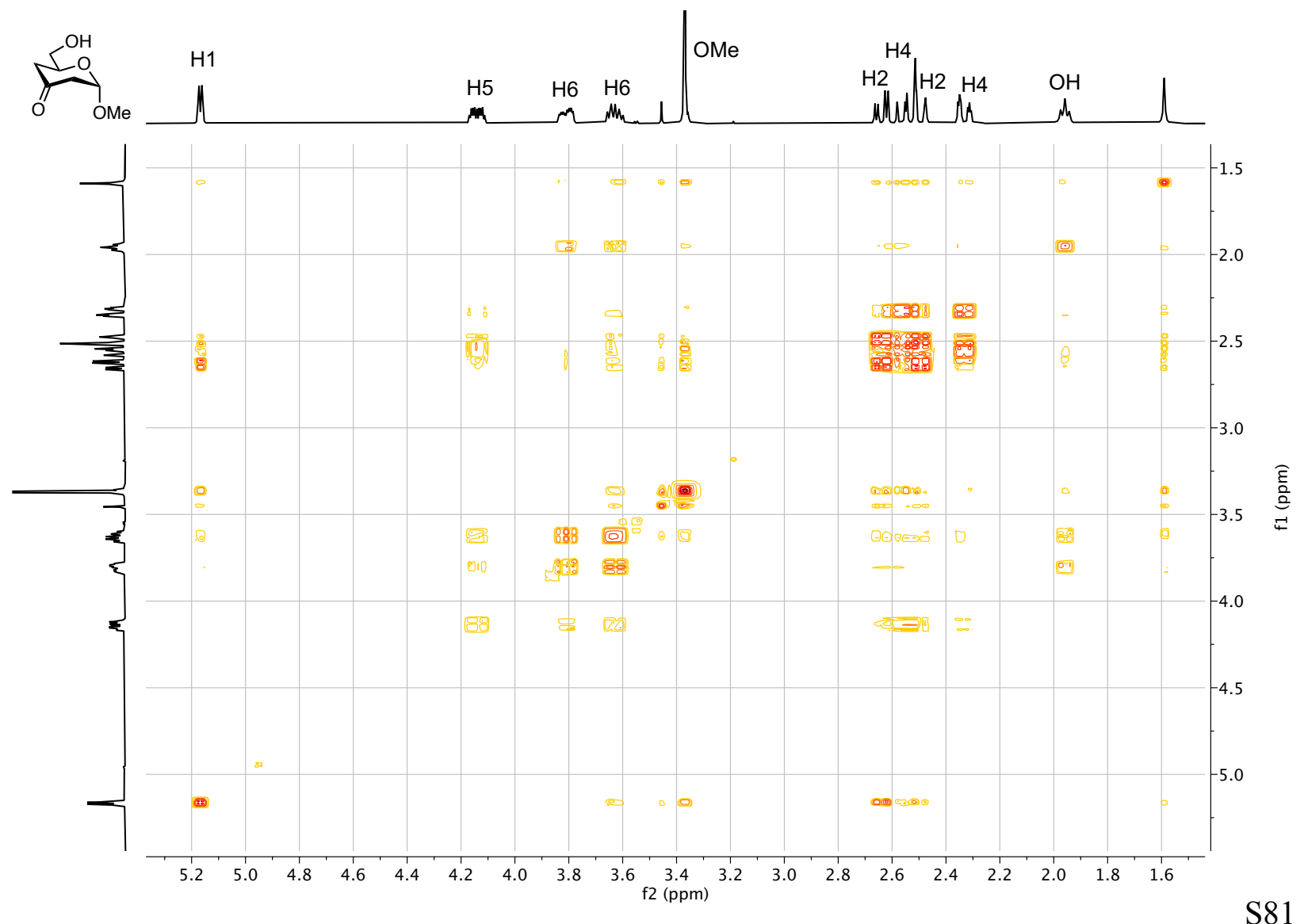


21

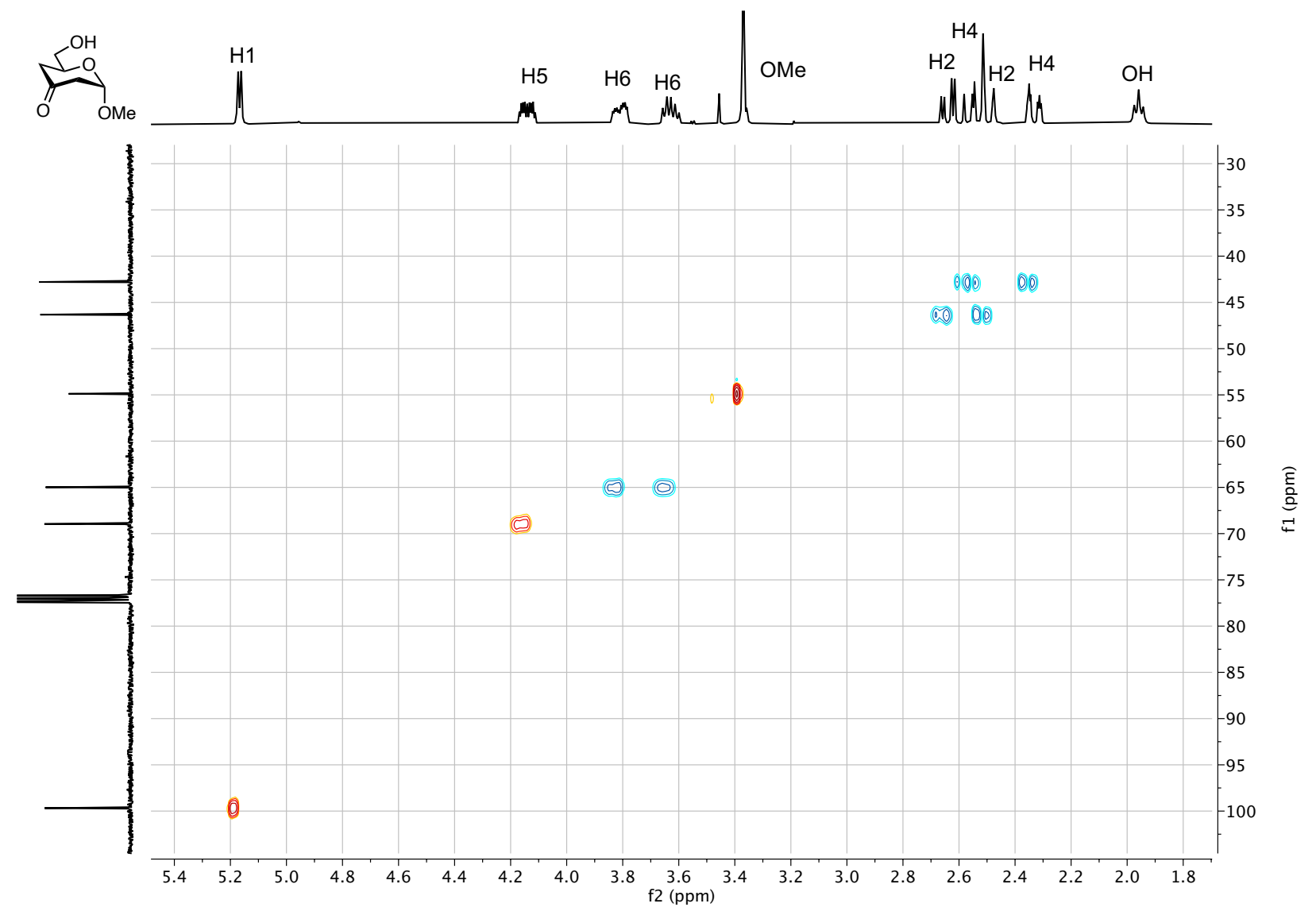

22
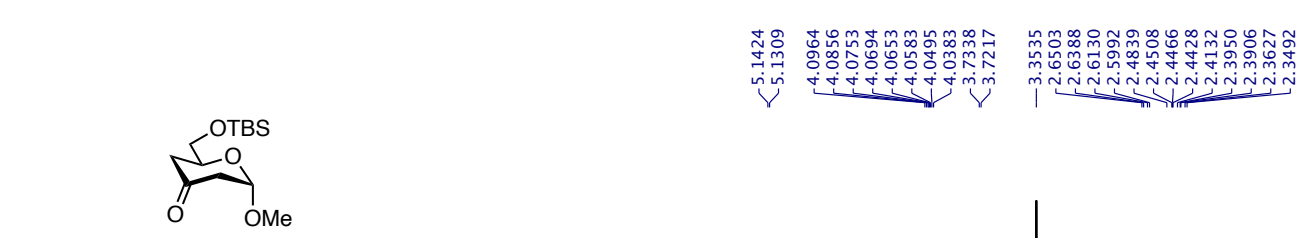

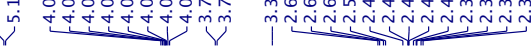


22

OMe

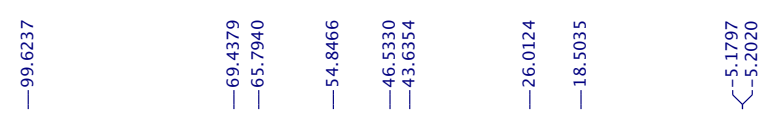
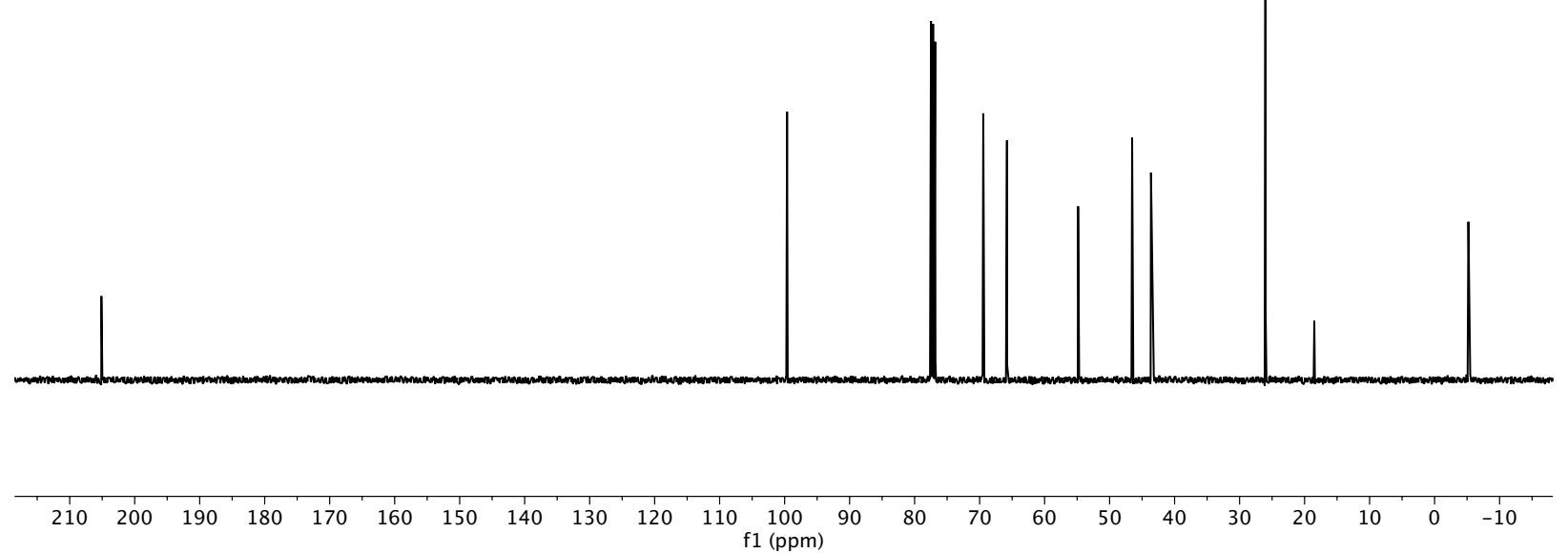

22

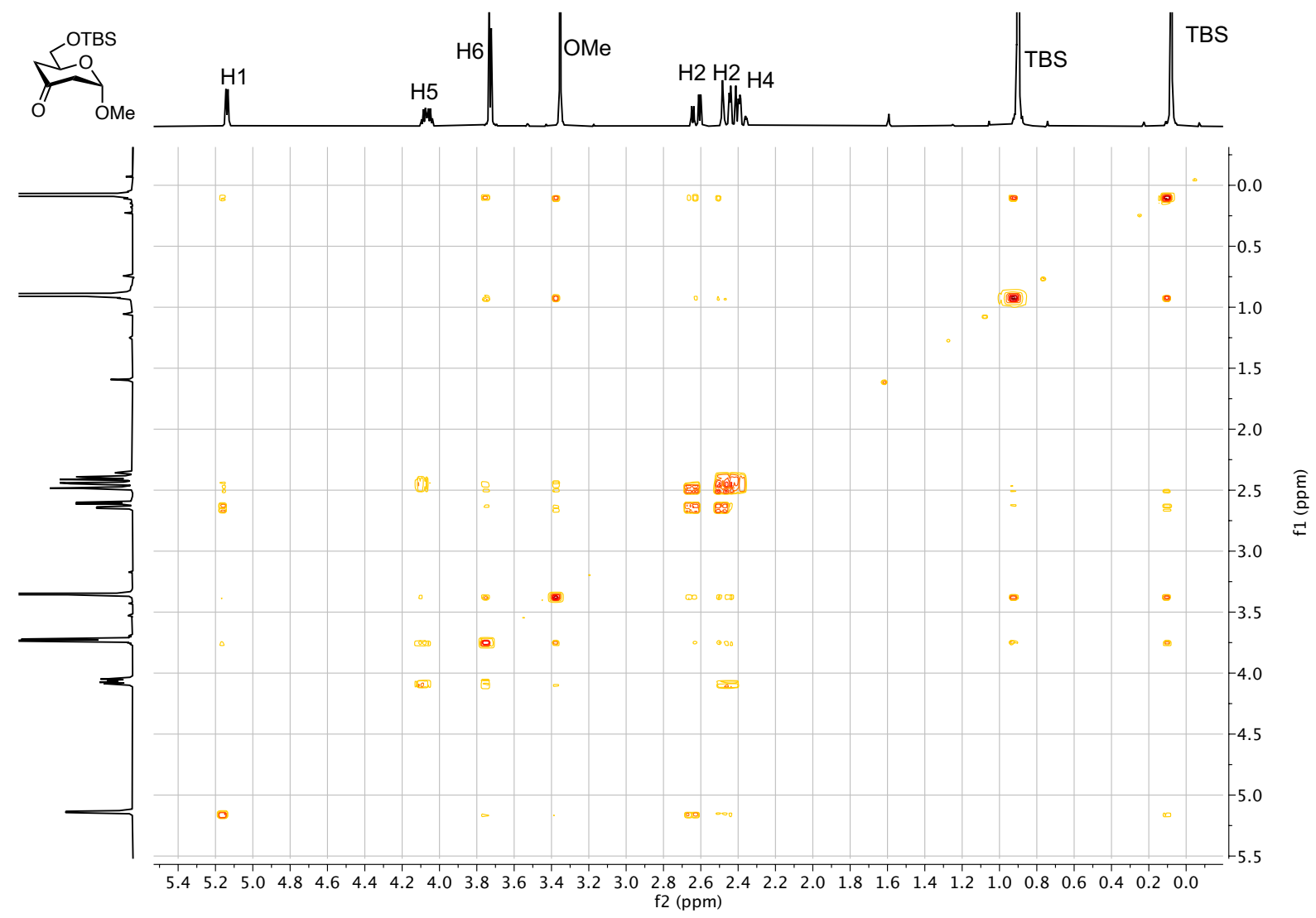




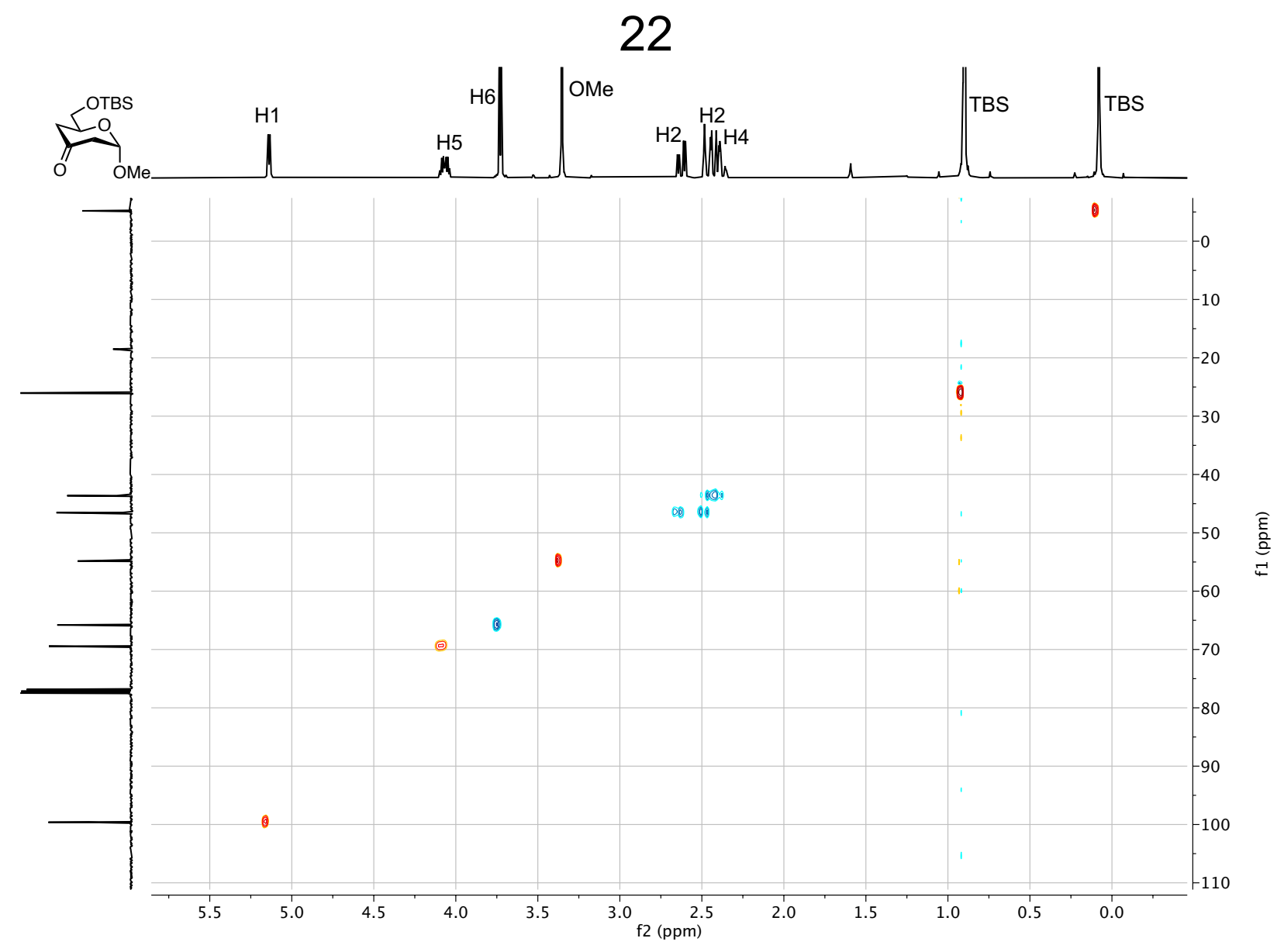

S12

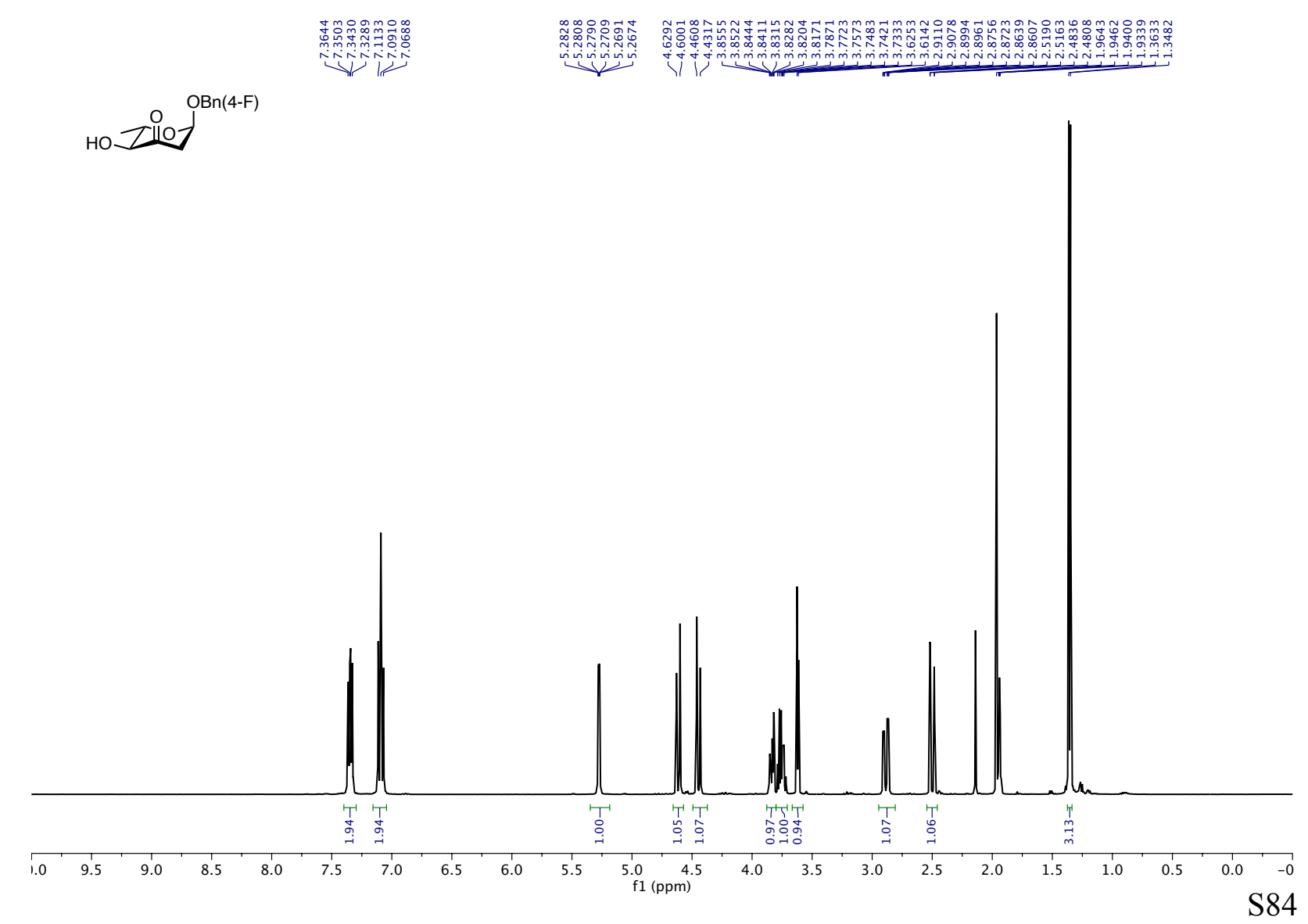




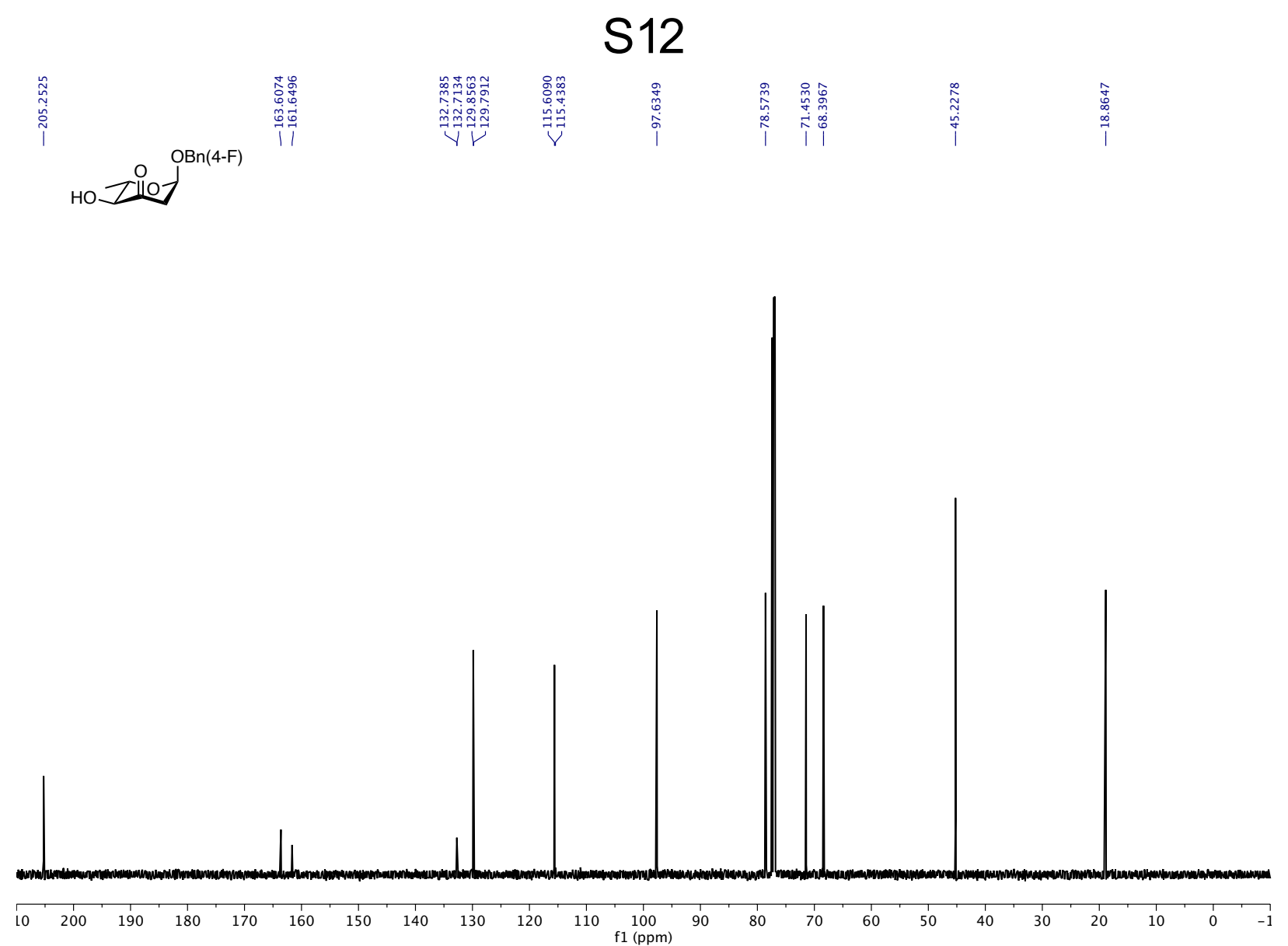

S12

nо 기이
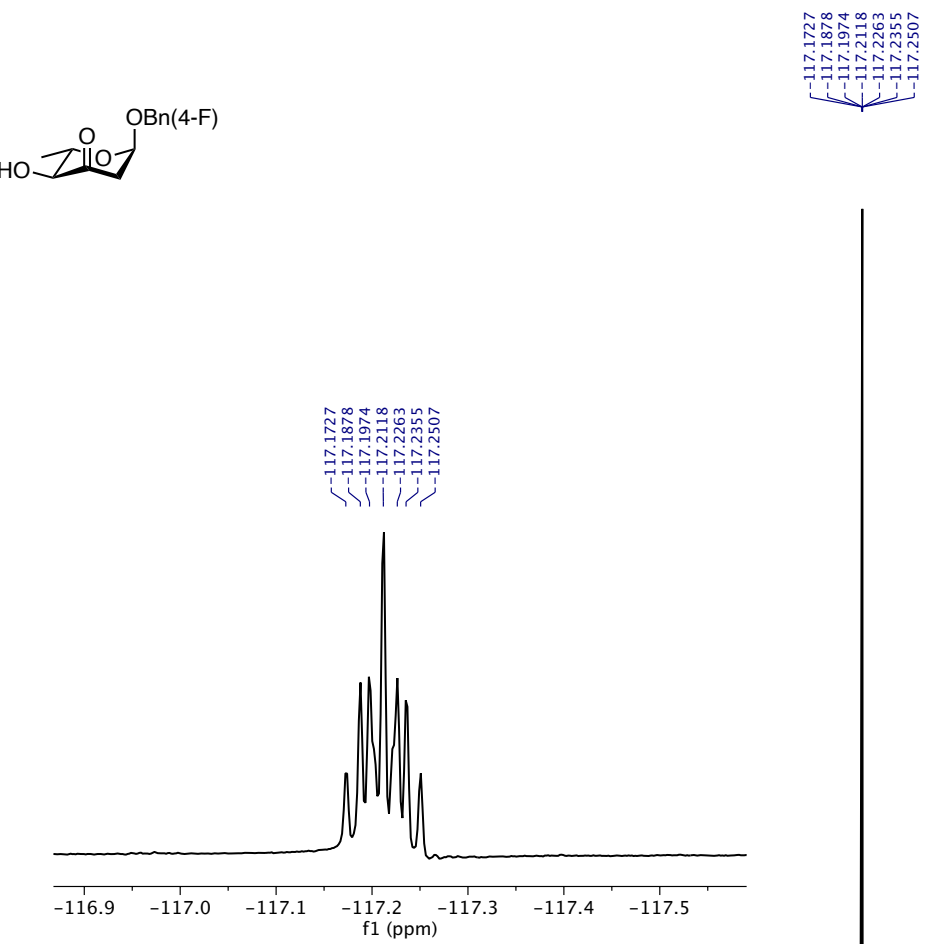

\begin{tabular}{llllllllllllllllllllllll}
\hline 0 & 0 & -10 & -20 & -30 & -40 & -50 & -60 & -70 & -80 & -90 & -100 & -110 & -120 & -130 & -140 & -150 & -160 & -170 & -180 & -190 & -200 \\
\hline
\end{tabular} 


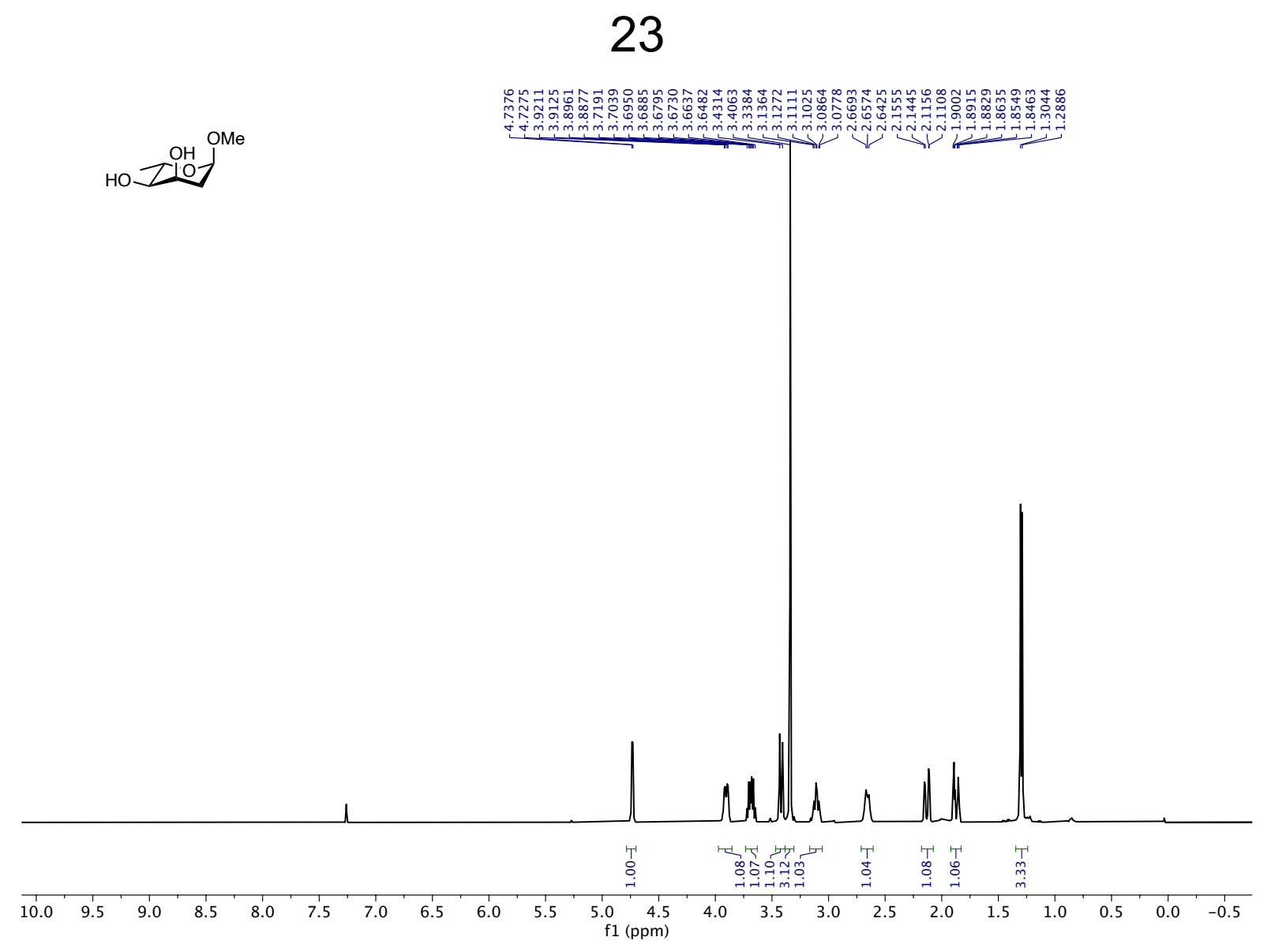

23

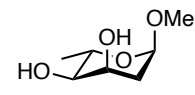

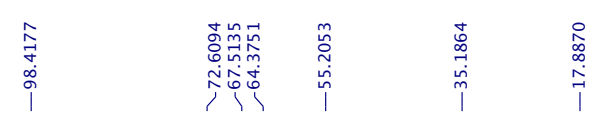

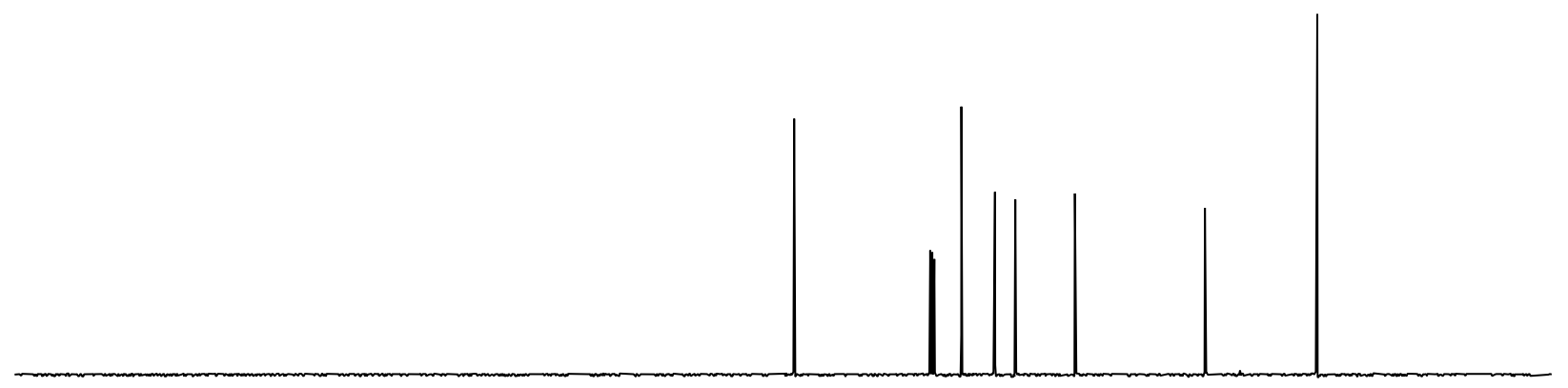




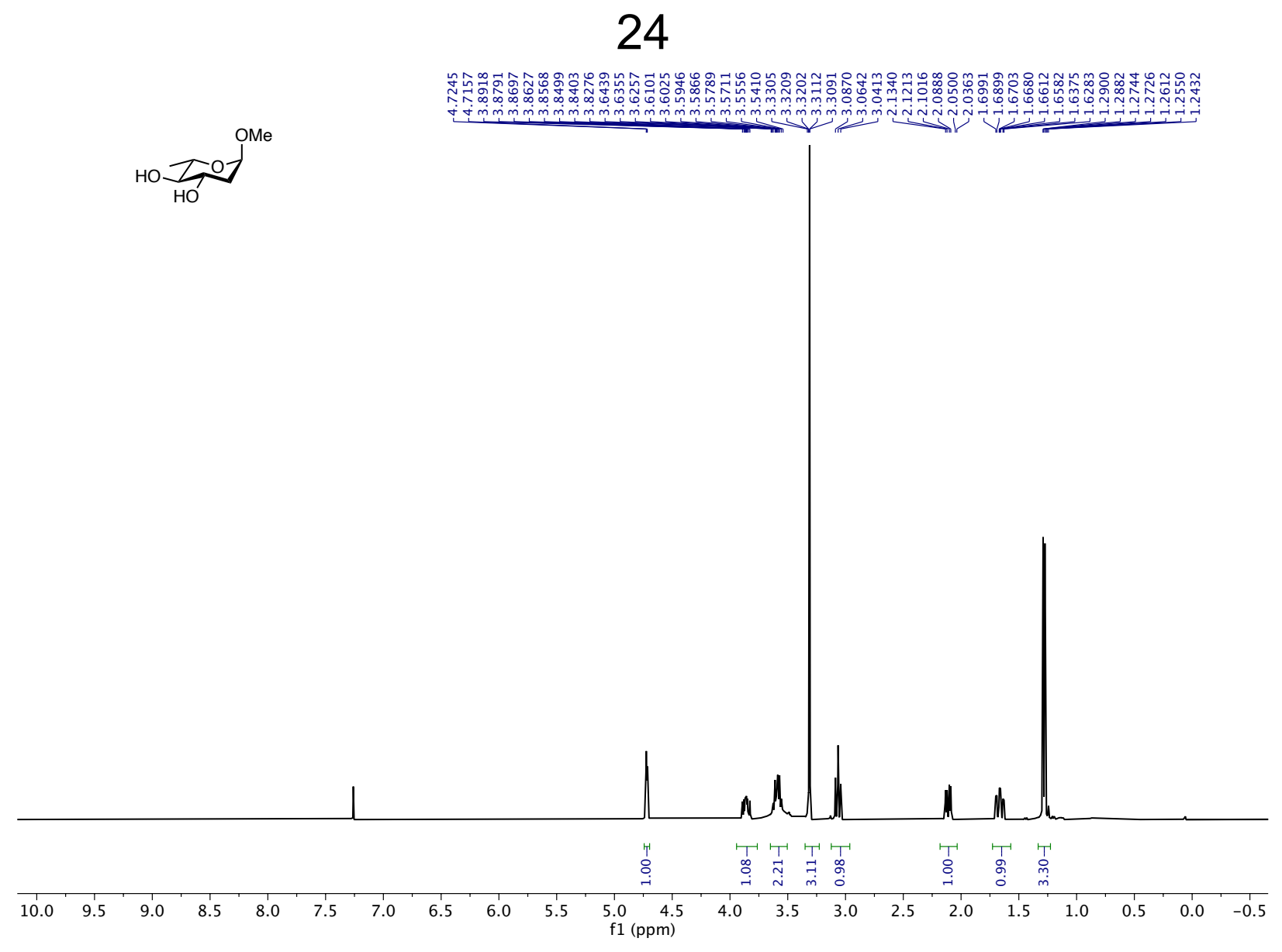

24

HO HO $_{\text {HO }}^{\text {OMe }}$

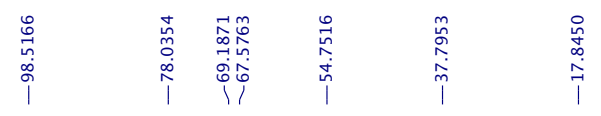

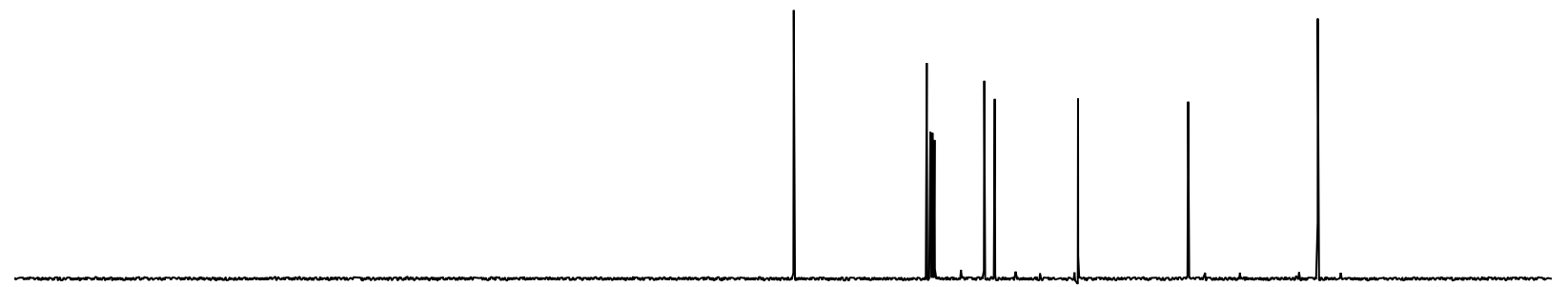

$\begin{array}{rllllllllllllllllllllll}210 & 200 & 190 & 180 & 170 & 160 & 150 & 140 & 130 & 120 & 110 & \begin{array}{c}100 \\ \mathrm{f} 1(\mathrm{ppm})\end{array} & 90 & 80 & 70 & 60 & 50 & 40 & 30 & 20 & 10 & 0 & -10 \\ \mathrm{~S} 87\end{array}$ 


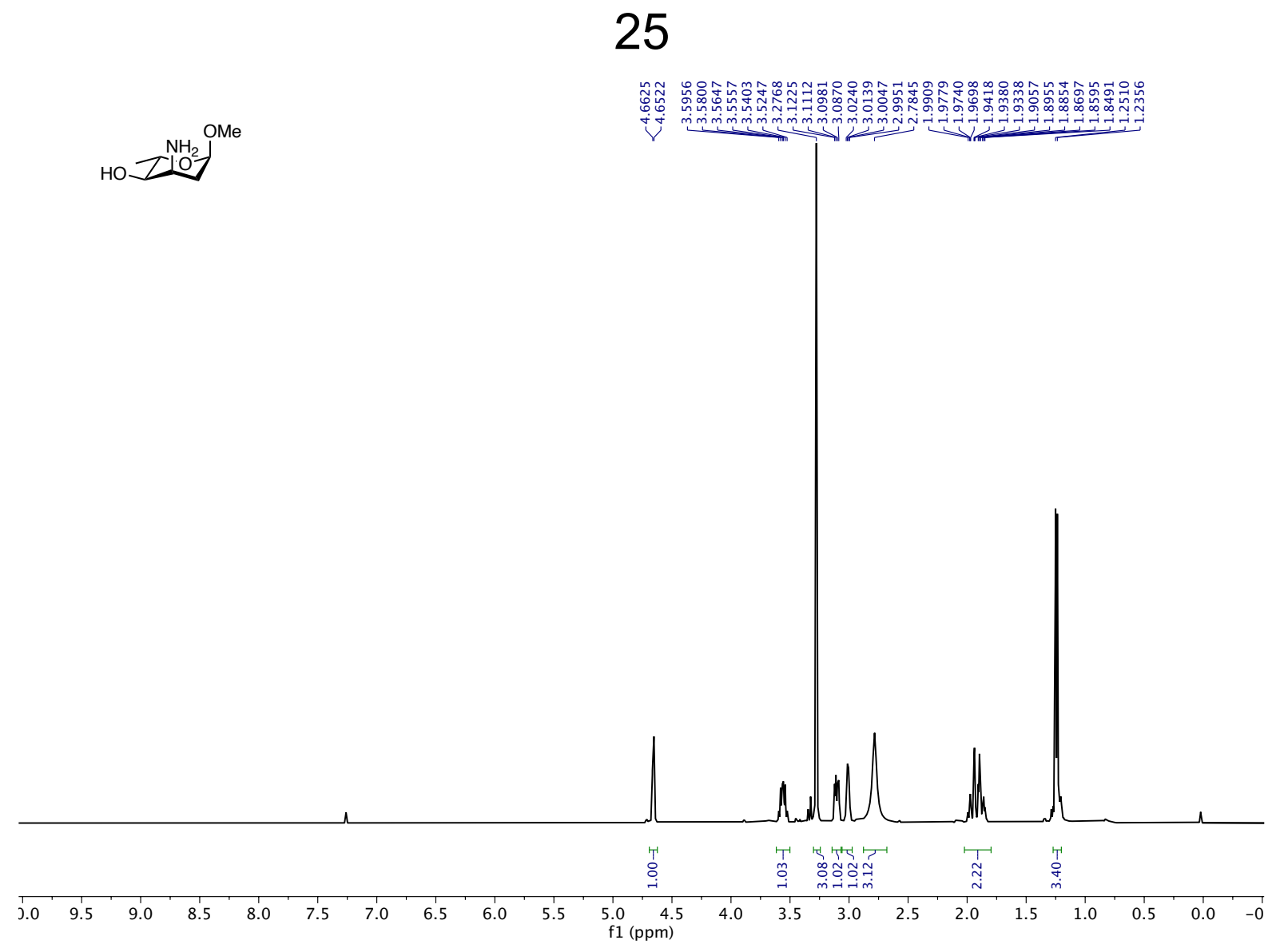

\section{5}

HO- $2 \cdot \mathrm{NH}^{2} \jmath^{\mathrm{OMe}}$

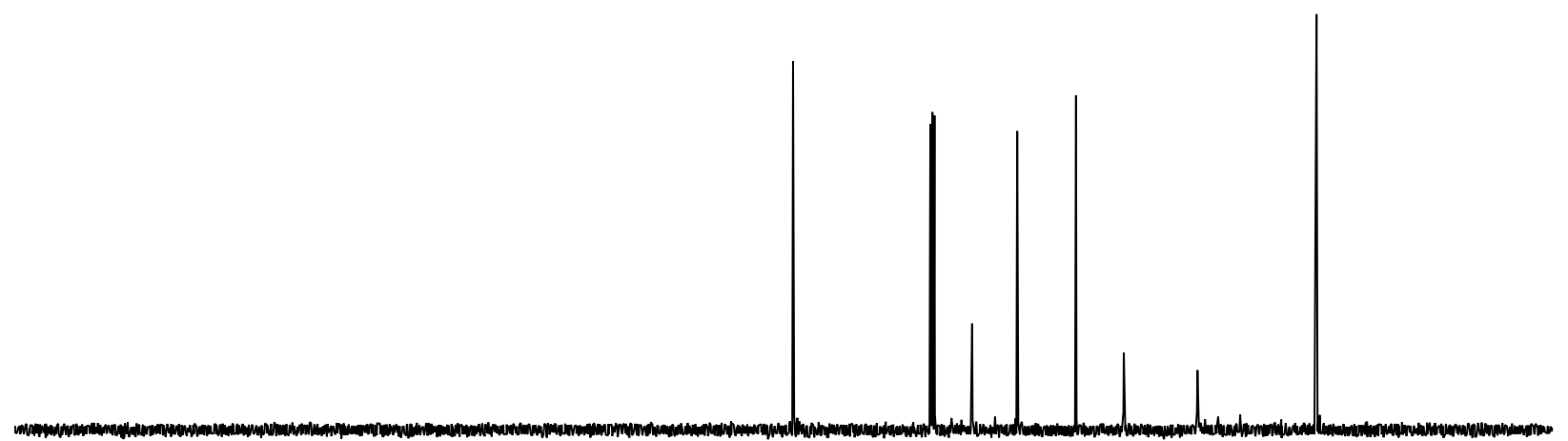




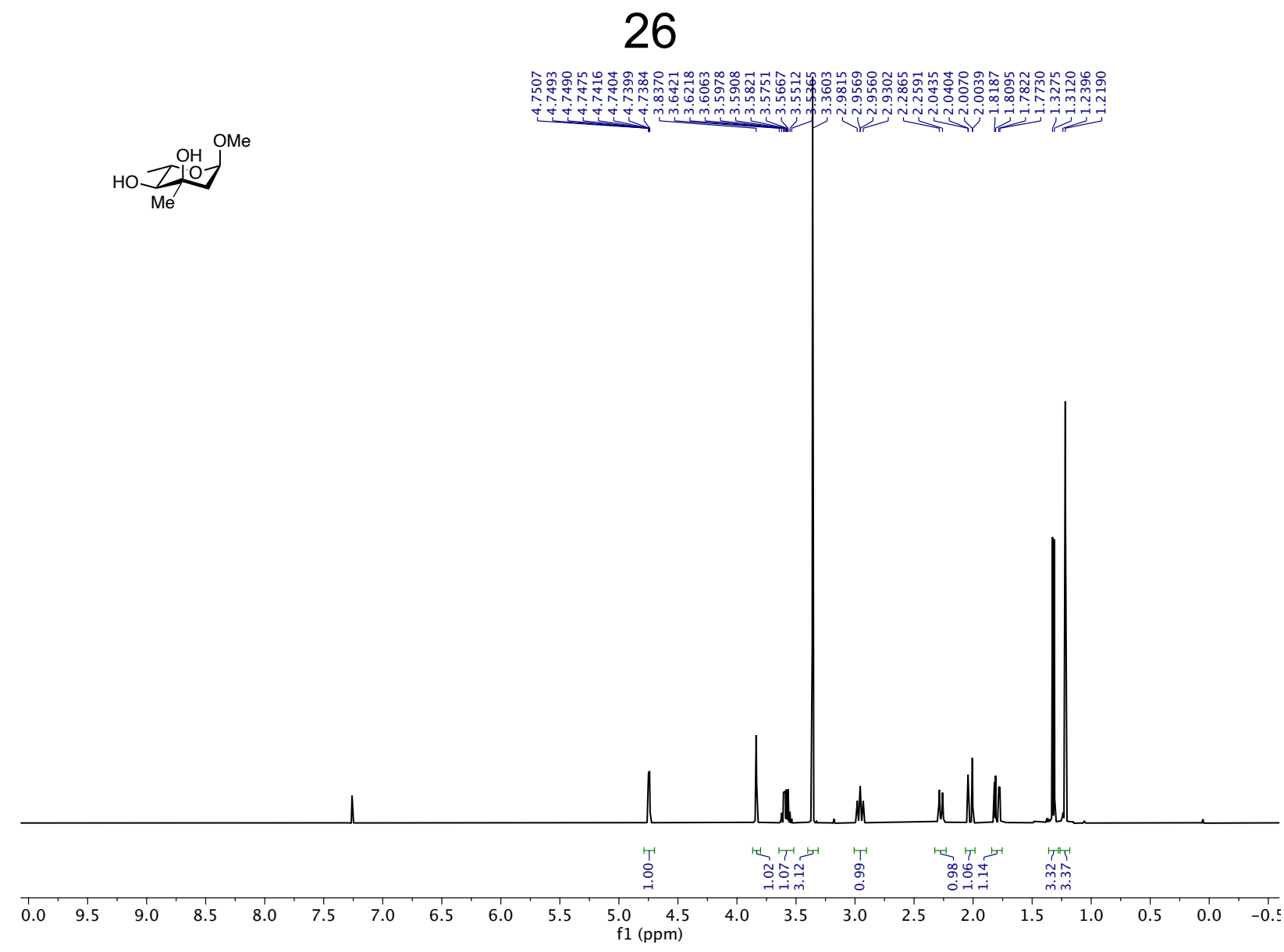

26

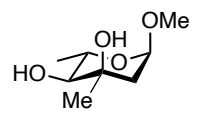

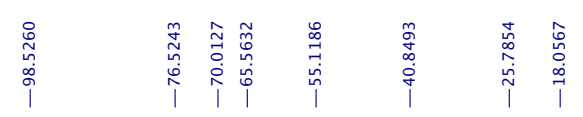
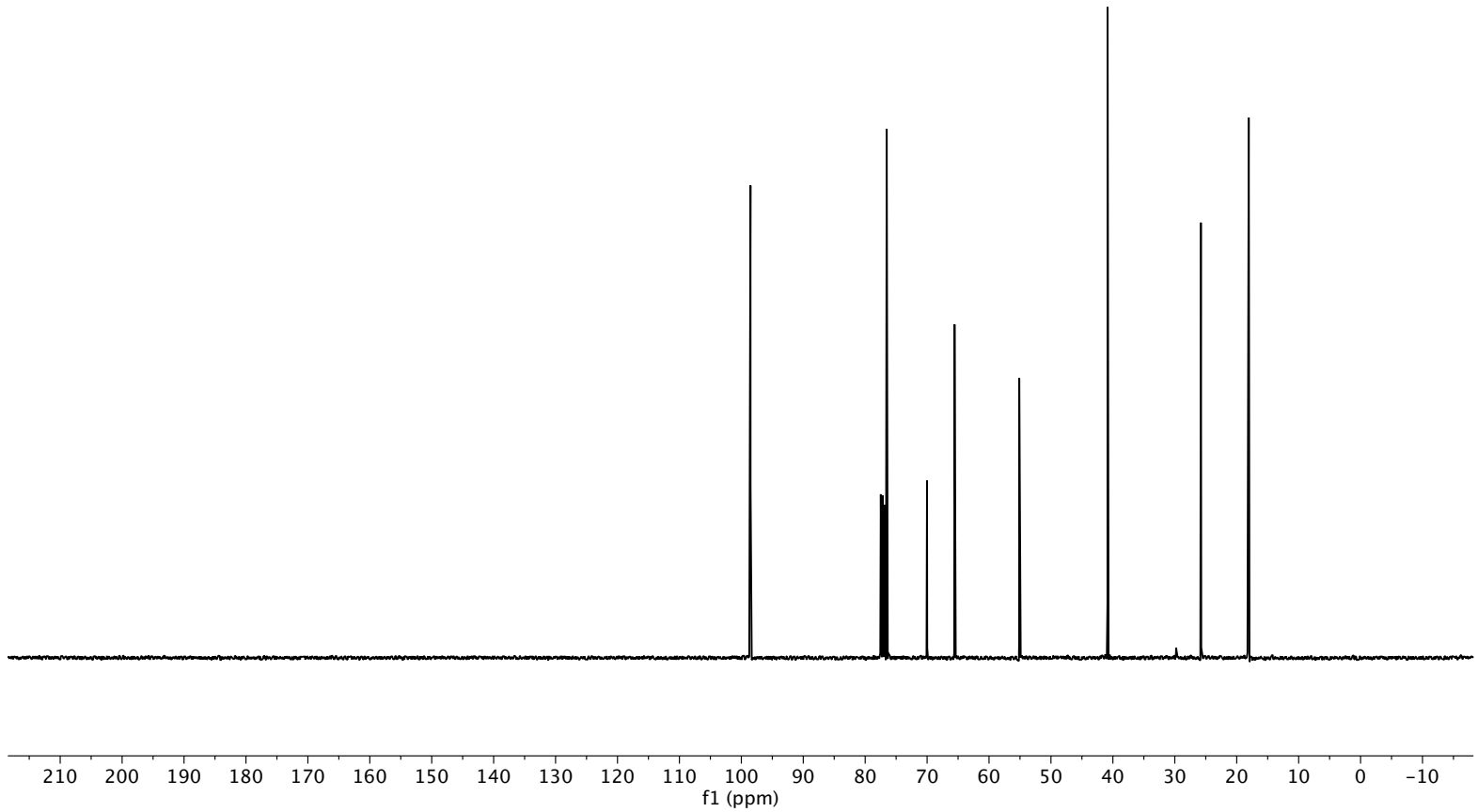


\section{S13}
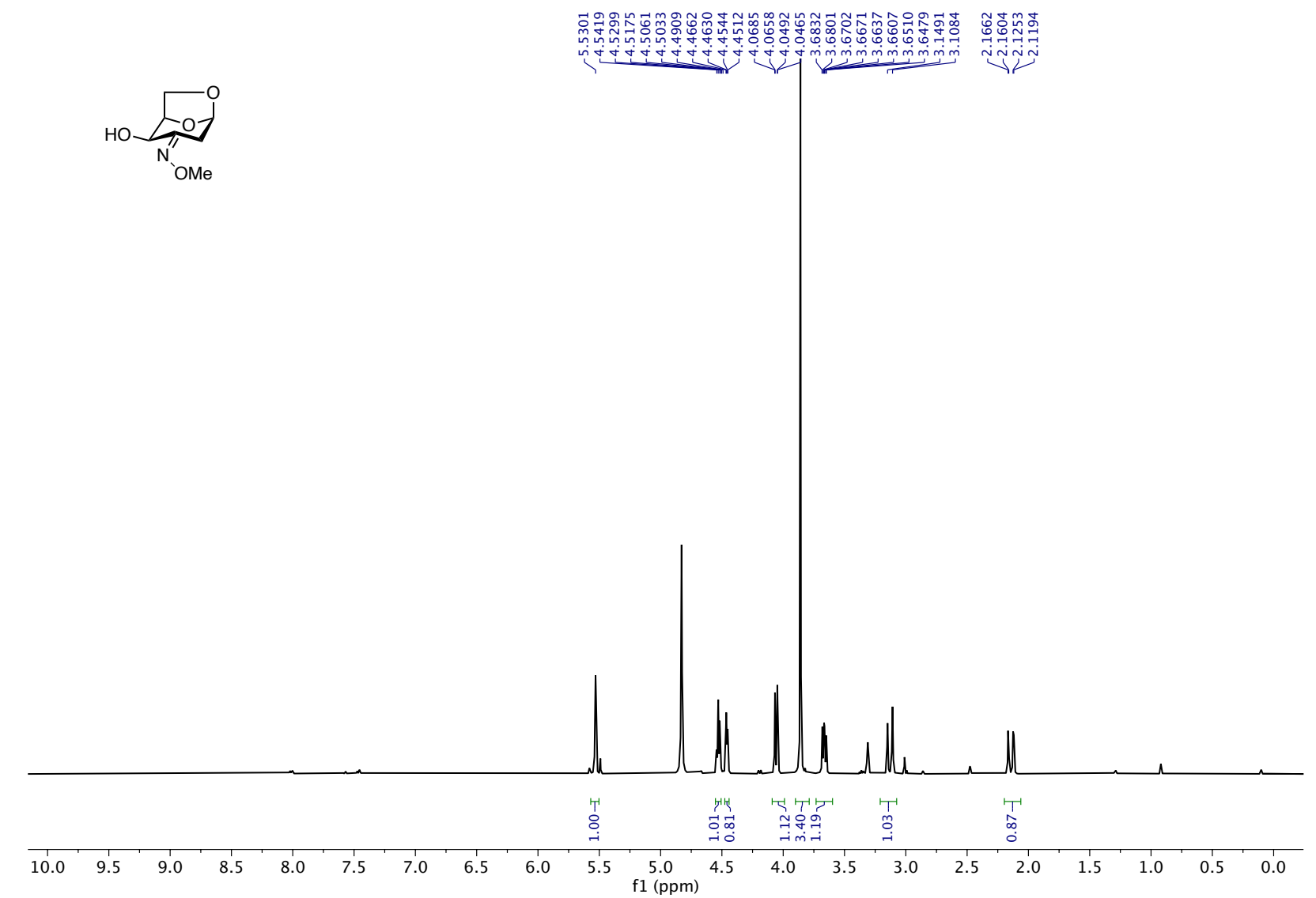

S13
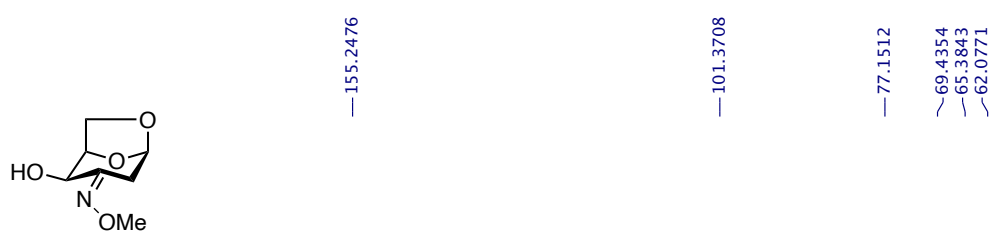

$\underset{\substack{\infty \\ \infty \\ \infty \\ \infty}}{\substack{\infty \\ m}}$

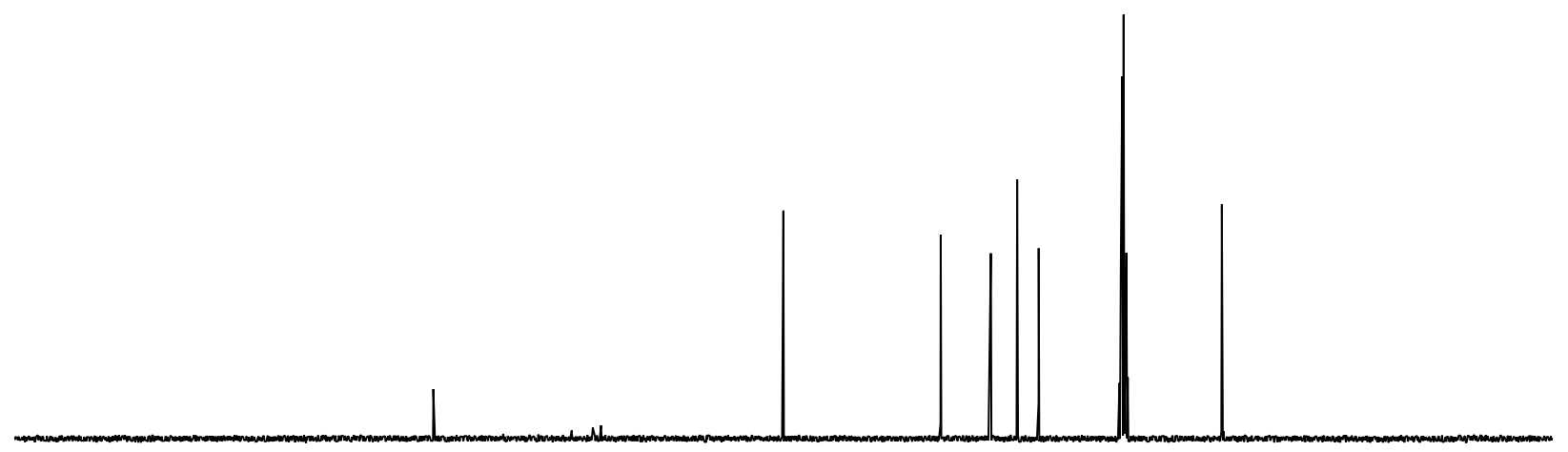

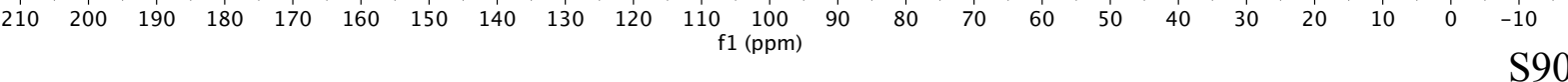




\section{S13}

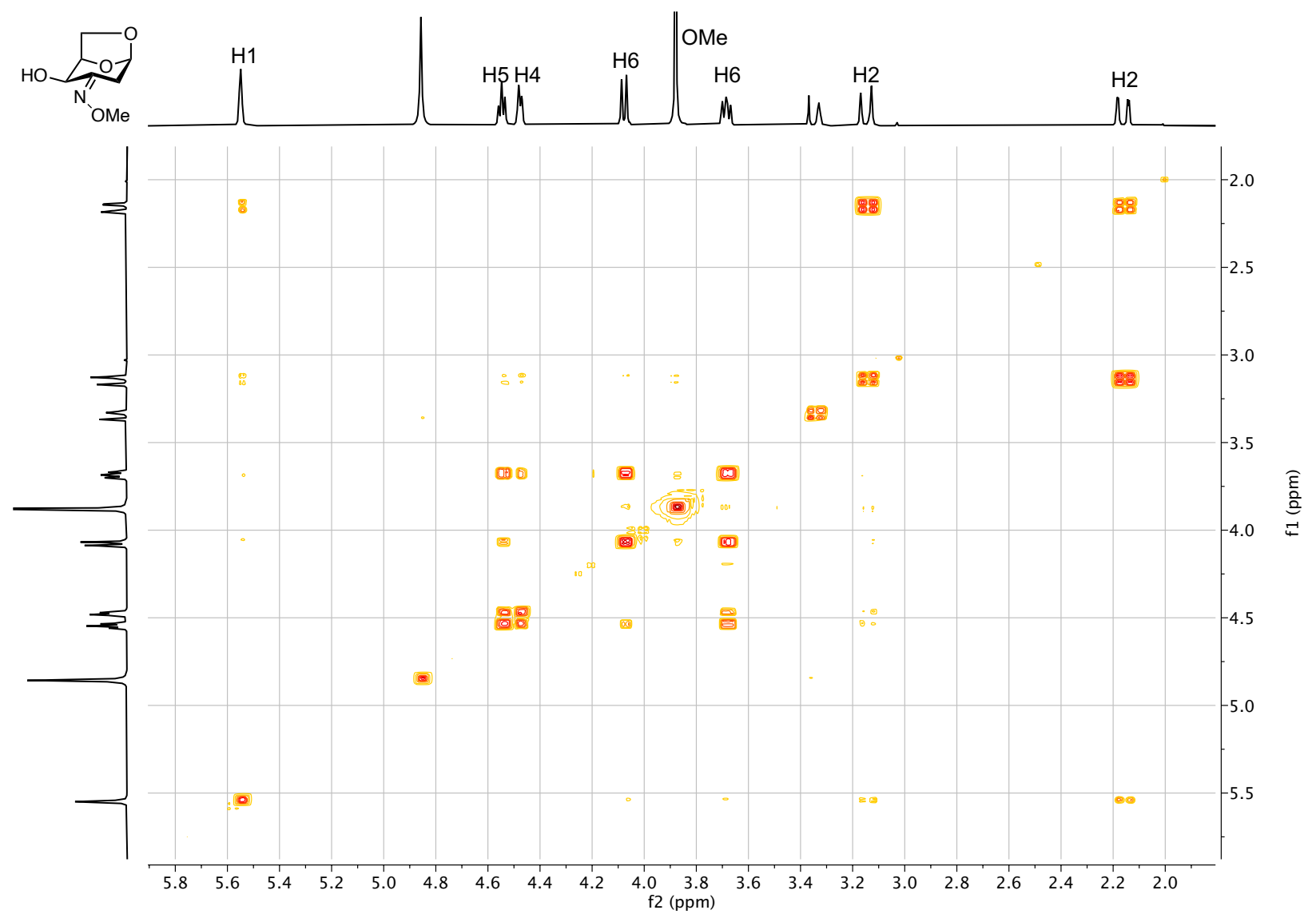

S13

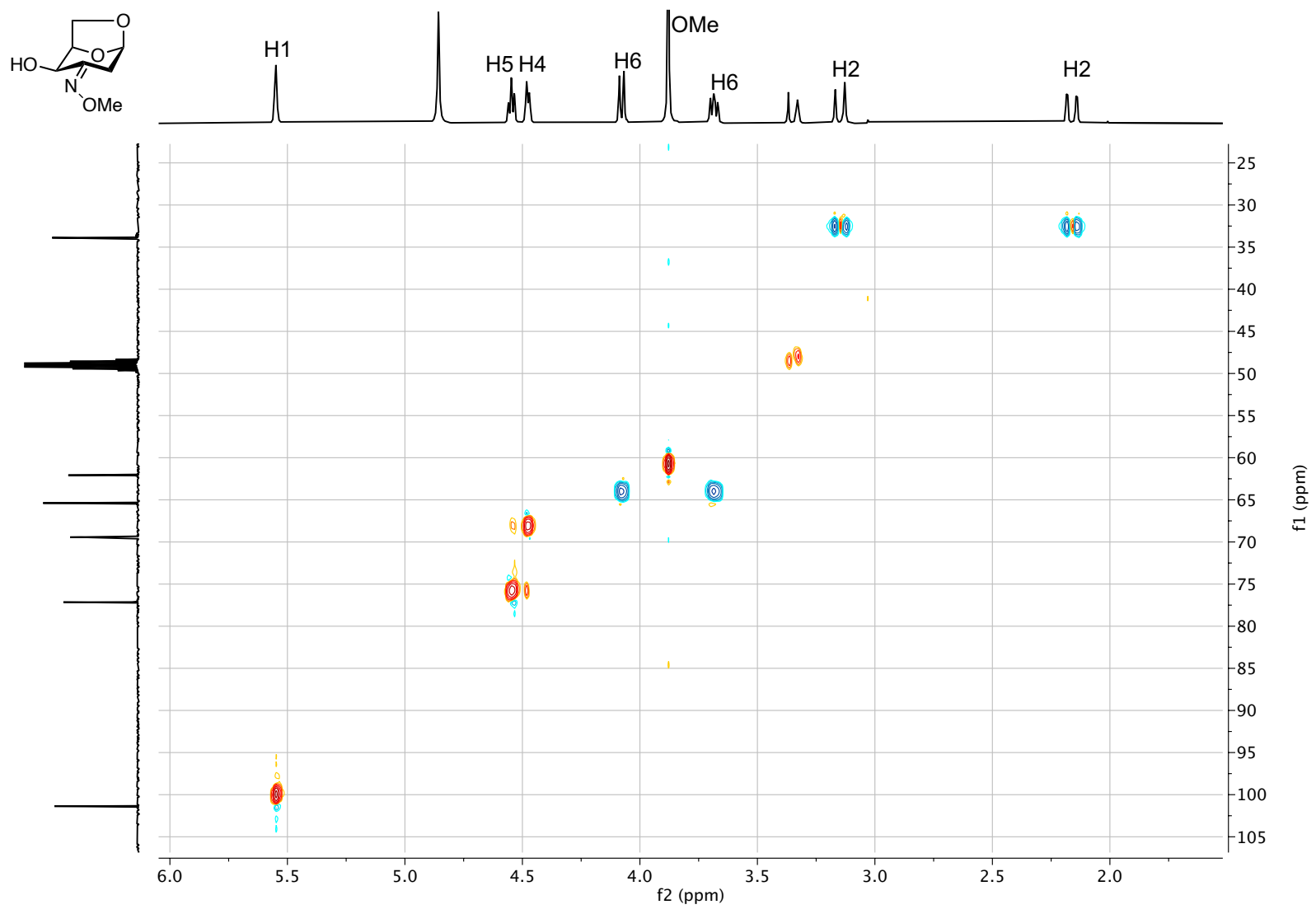




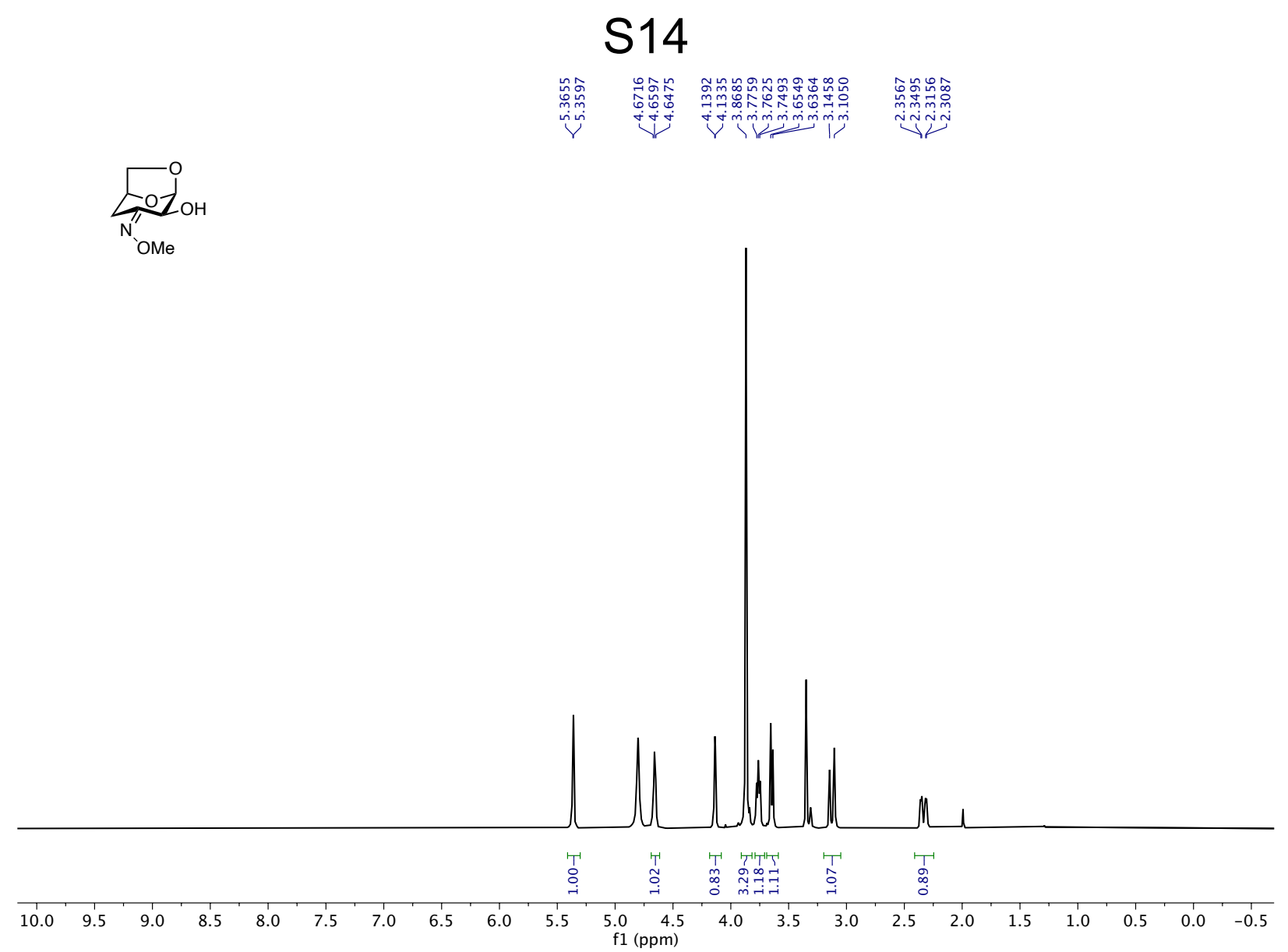

\section{S14}
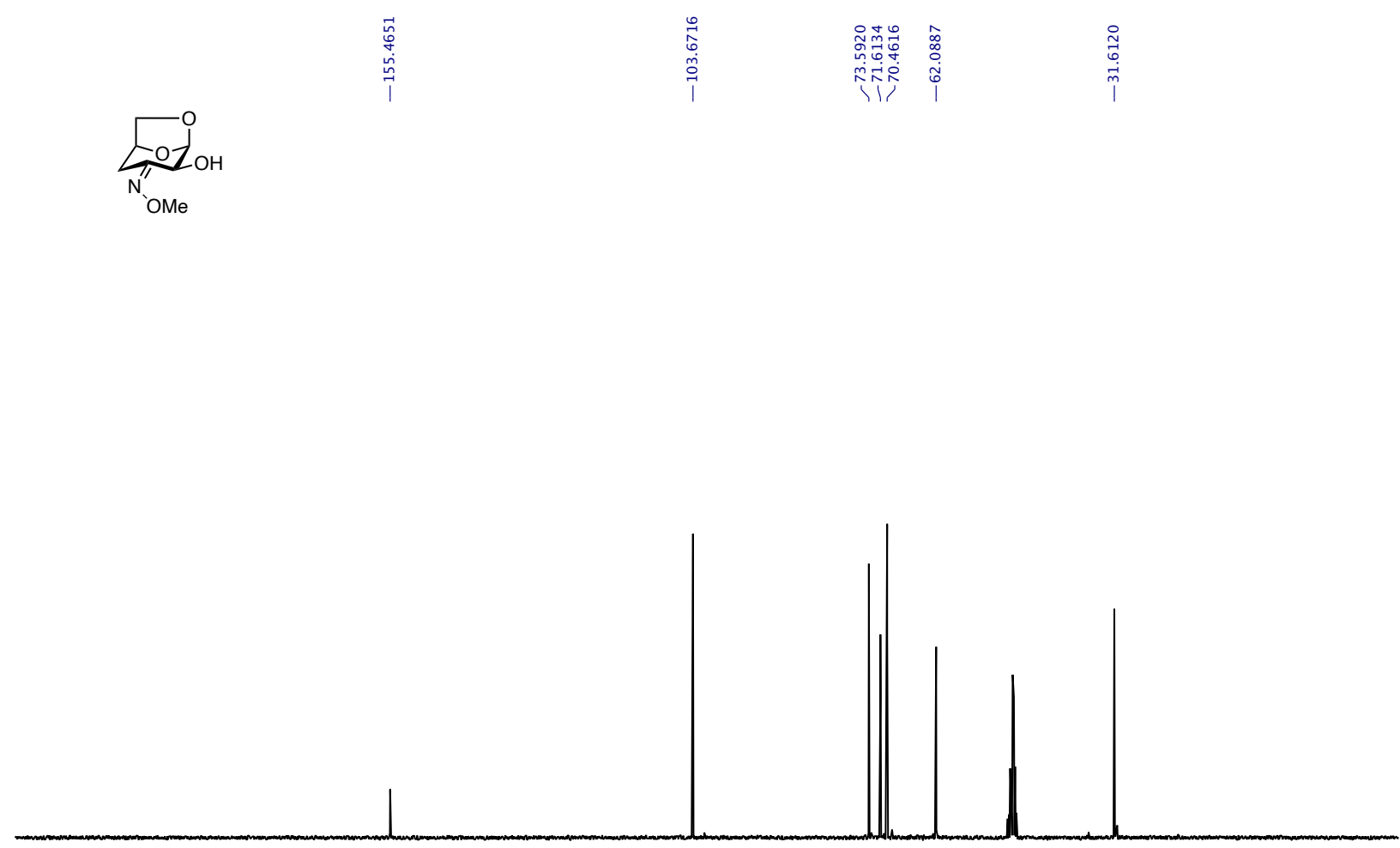

$\begin{array}{llllllllllllllllllllllll}210 & 200 & 190 & 180 & 170 & 160 & 150 & 140 & 130 & 120 & \begin{array}{c}110 \\ \mathrm{f} 1(\mathrm{ppm})\end{array} & 90 & 80 & 70 & 60 & 50 & 40 & 30 & 20 & 10 & 0 & -10 \\ \mathrm{~S} 92\end{array}$ 


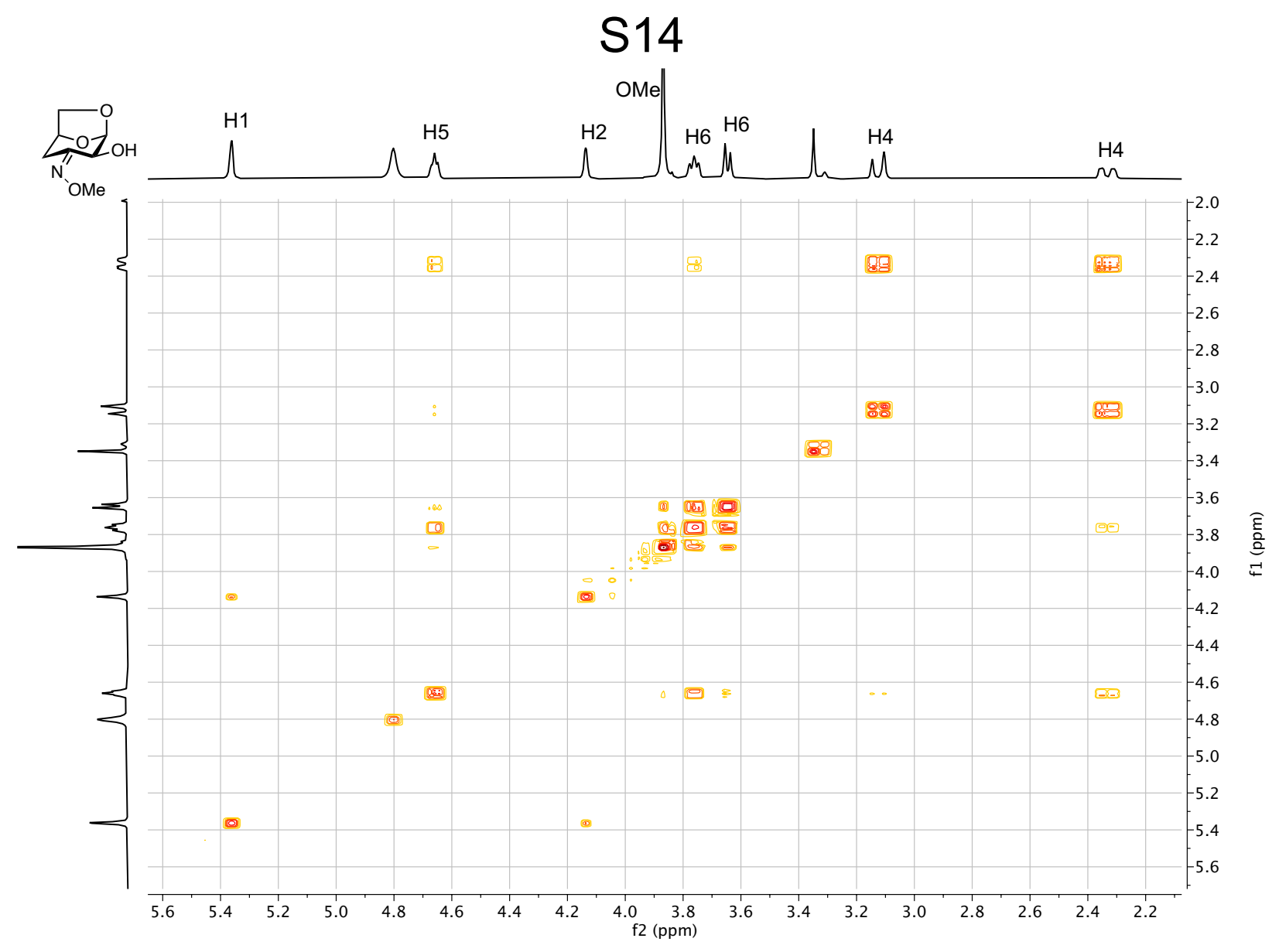

S14

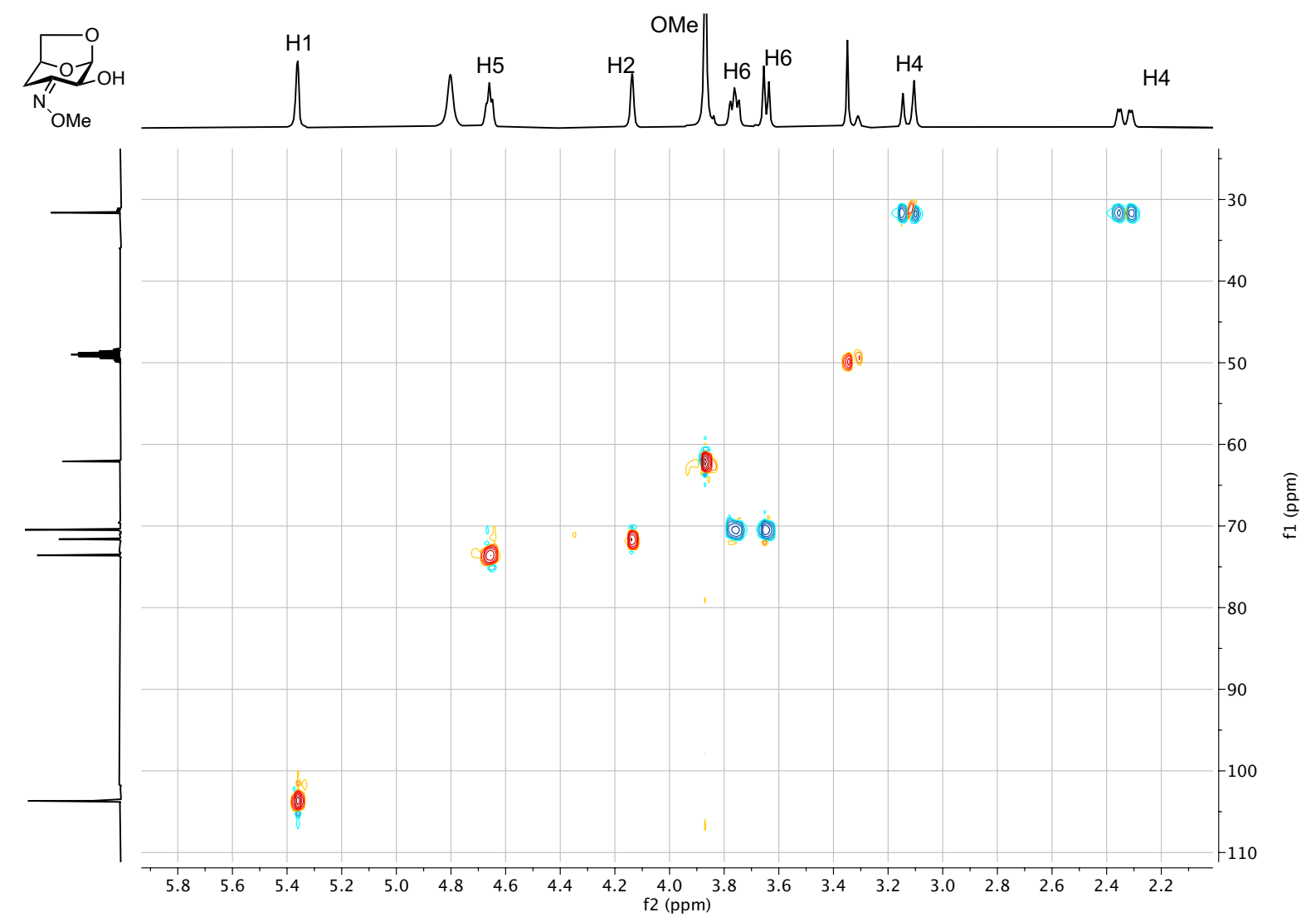




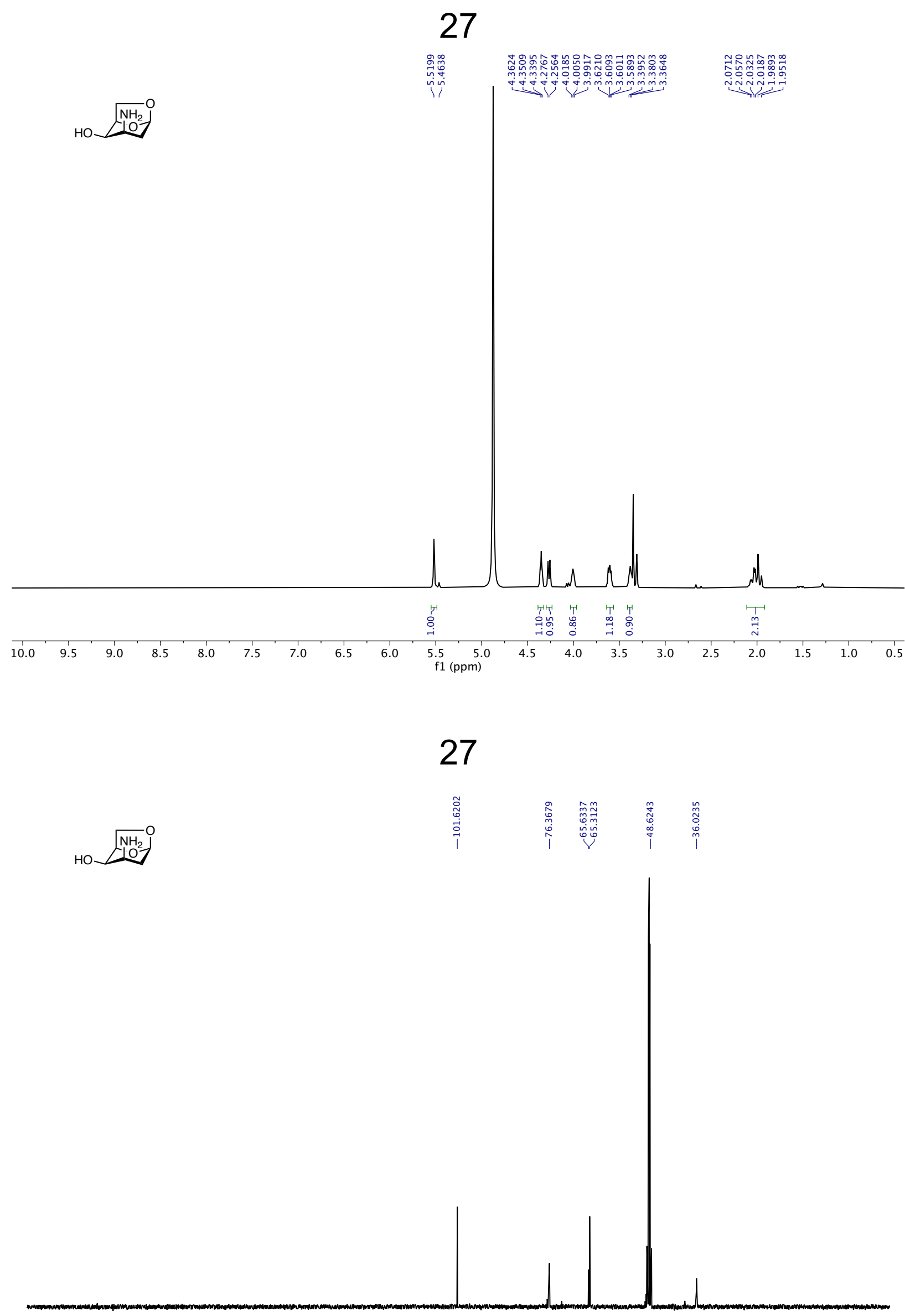

HON 
27

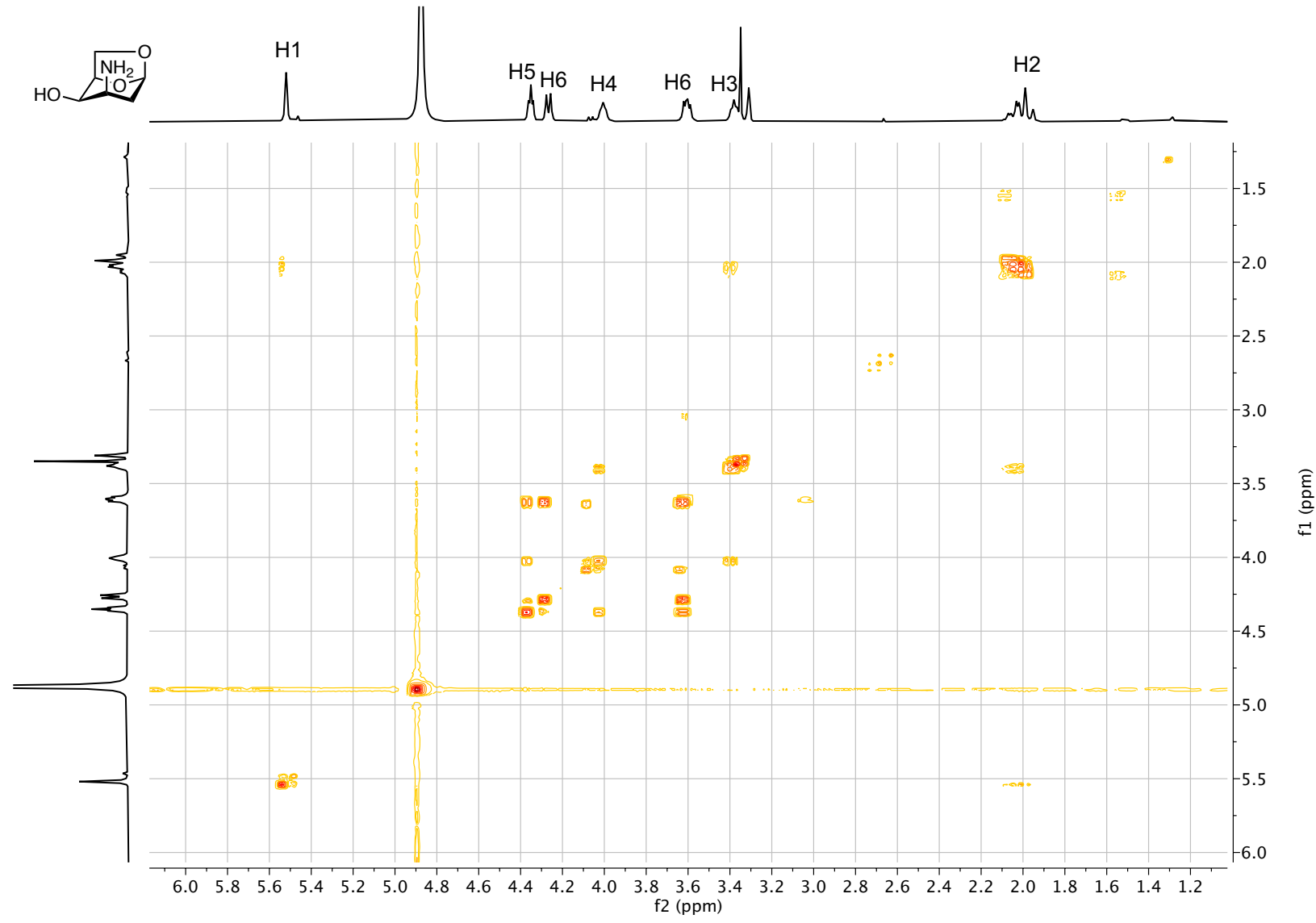

27

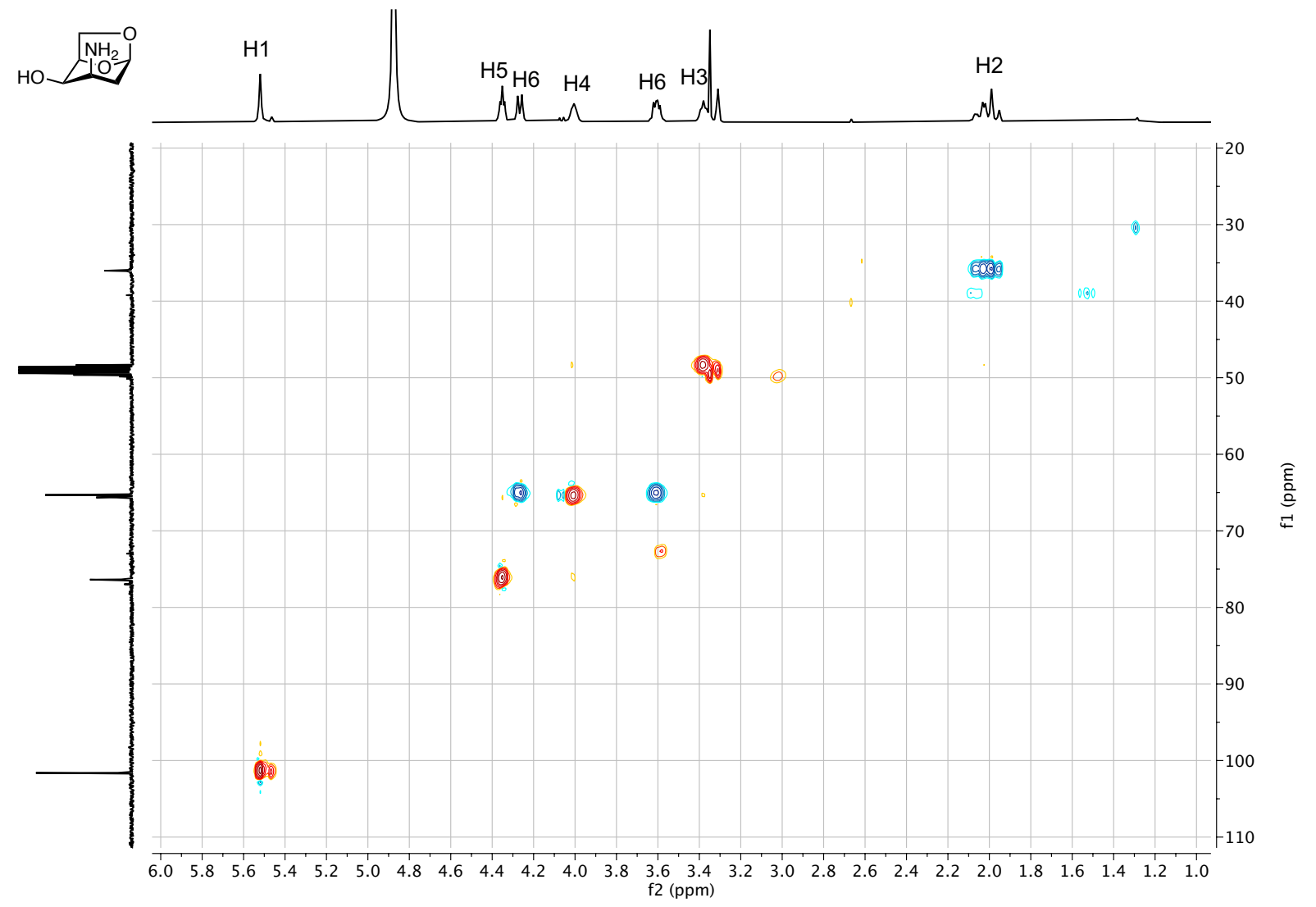


27

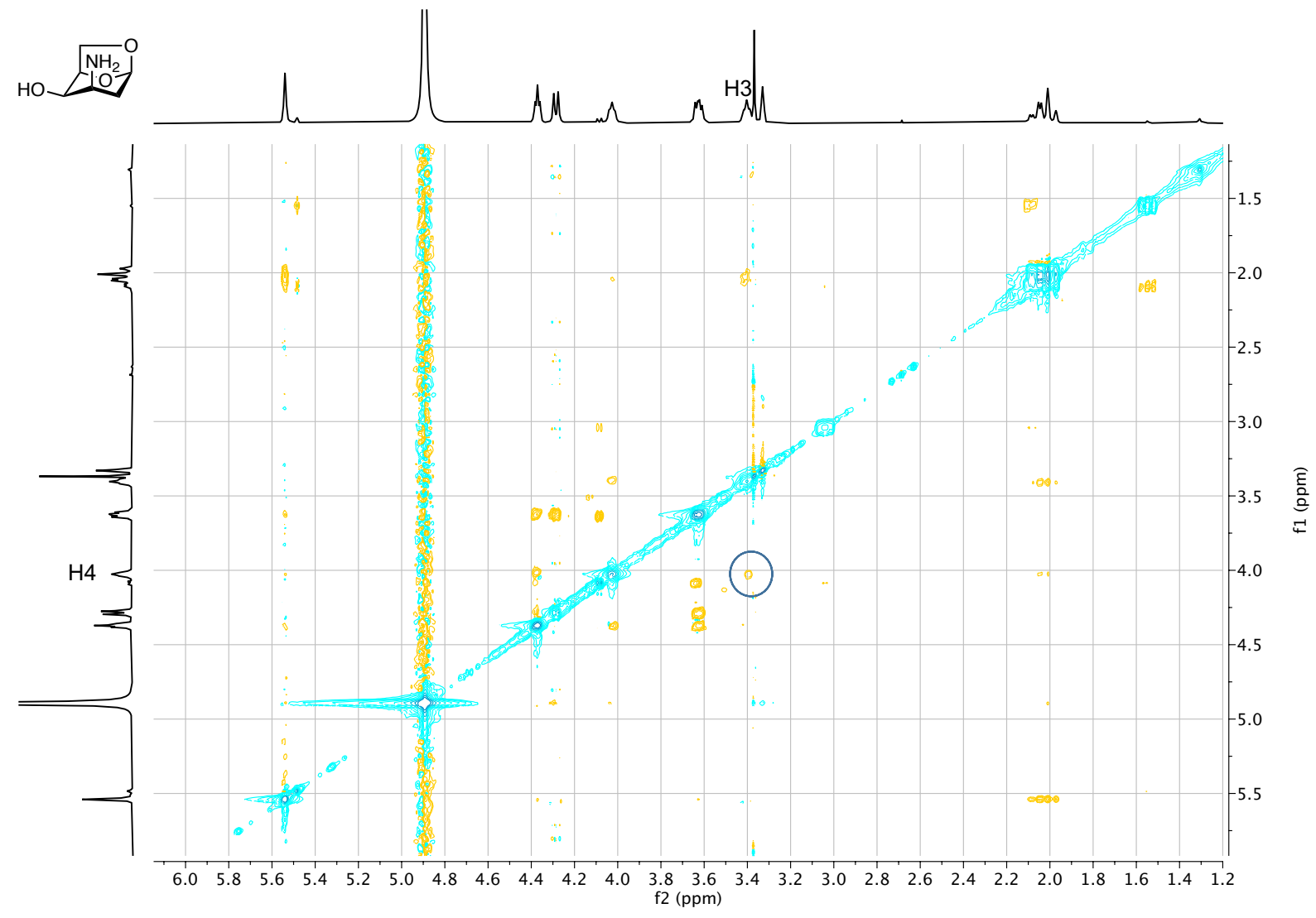

28

$\mathrm{NH}_{2} \mathrm{O}$

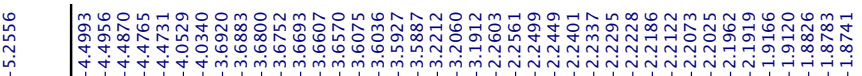

$20^{2} \mathrm{ZH}$

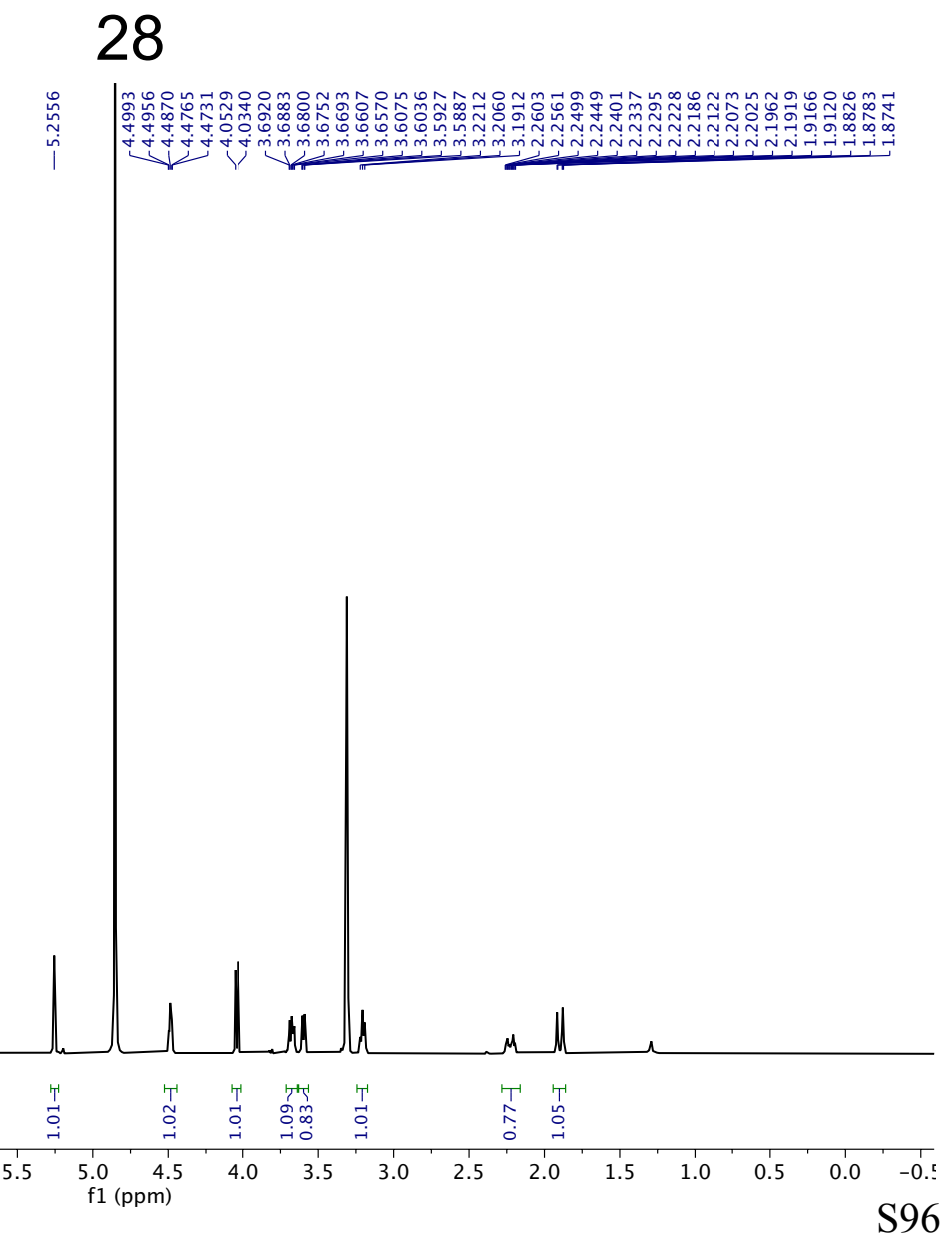




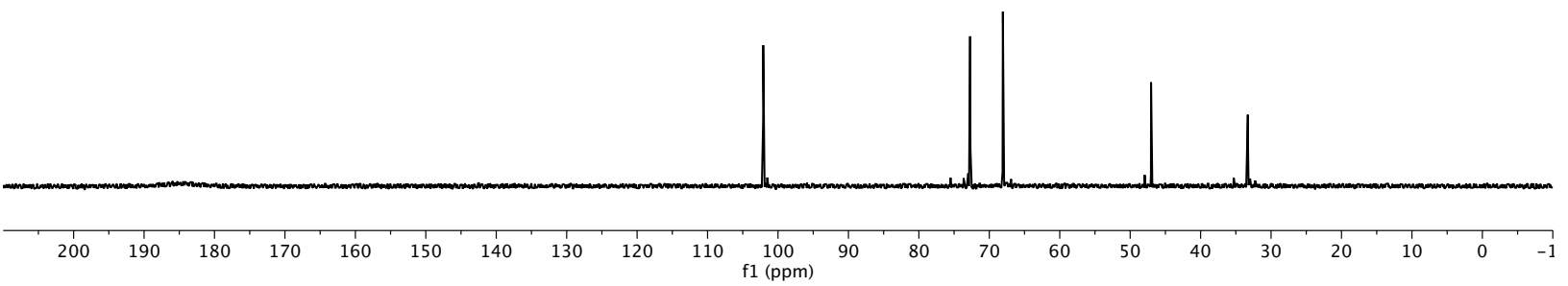

28

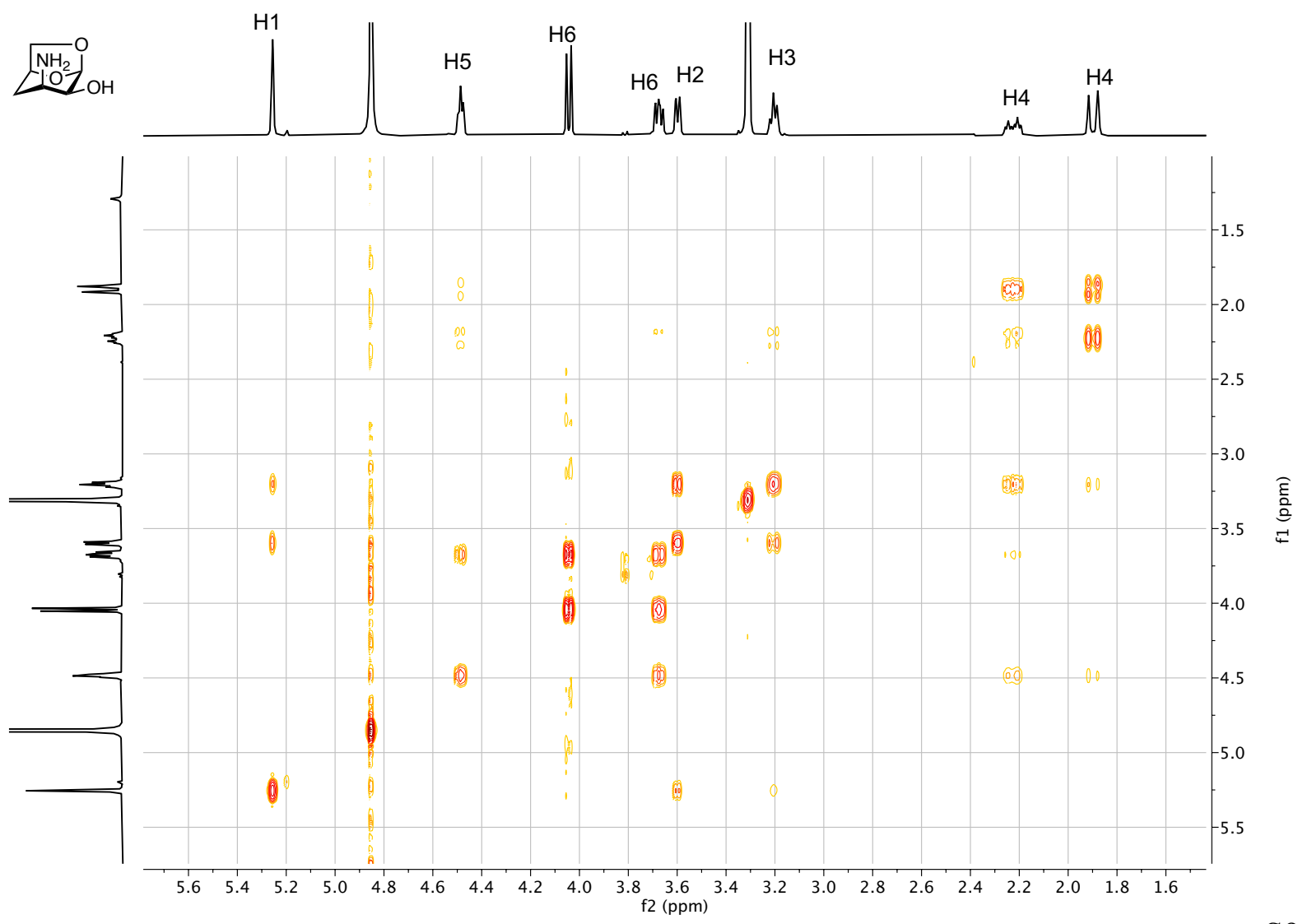


28

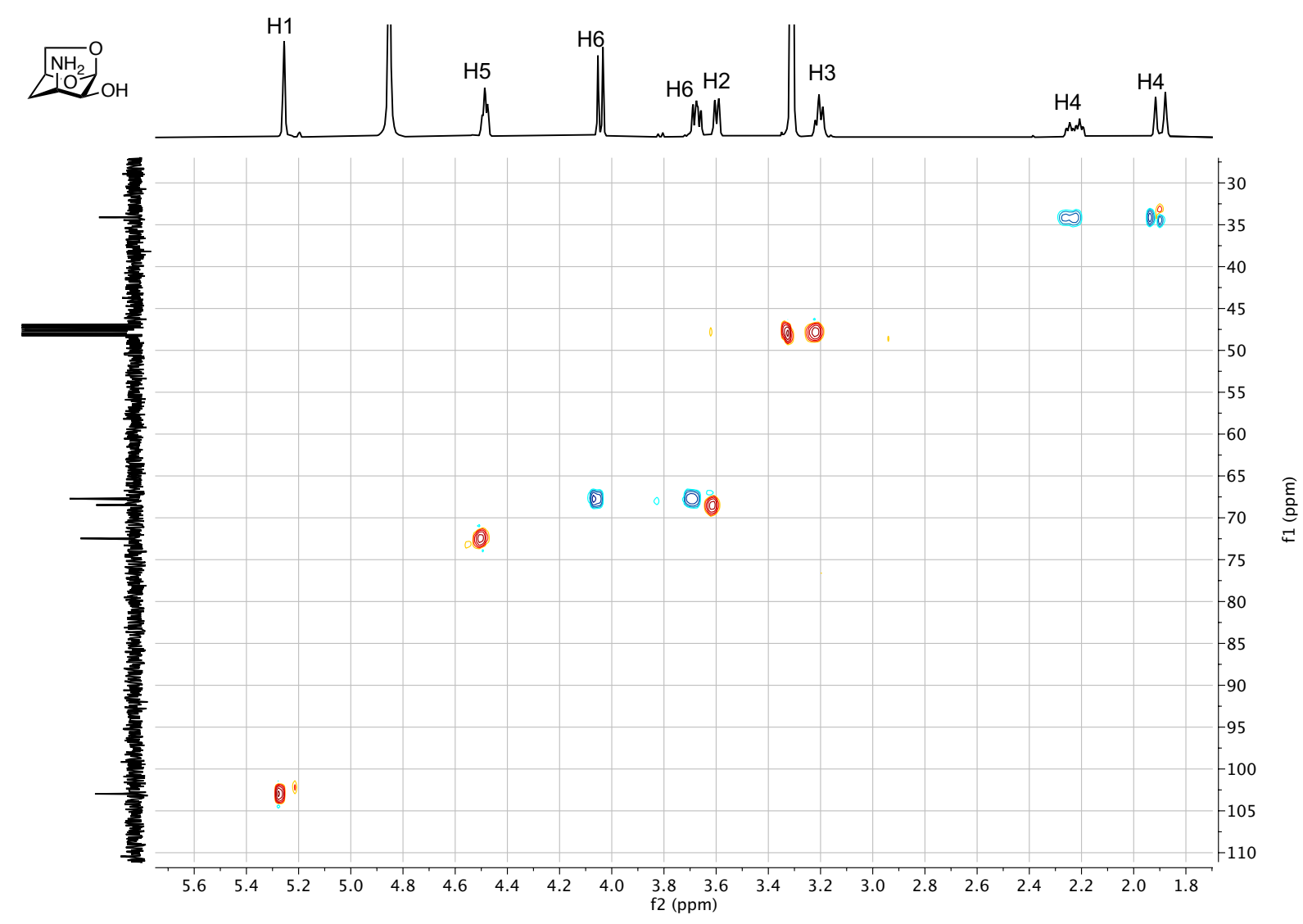

28

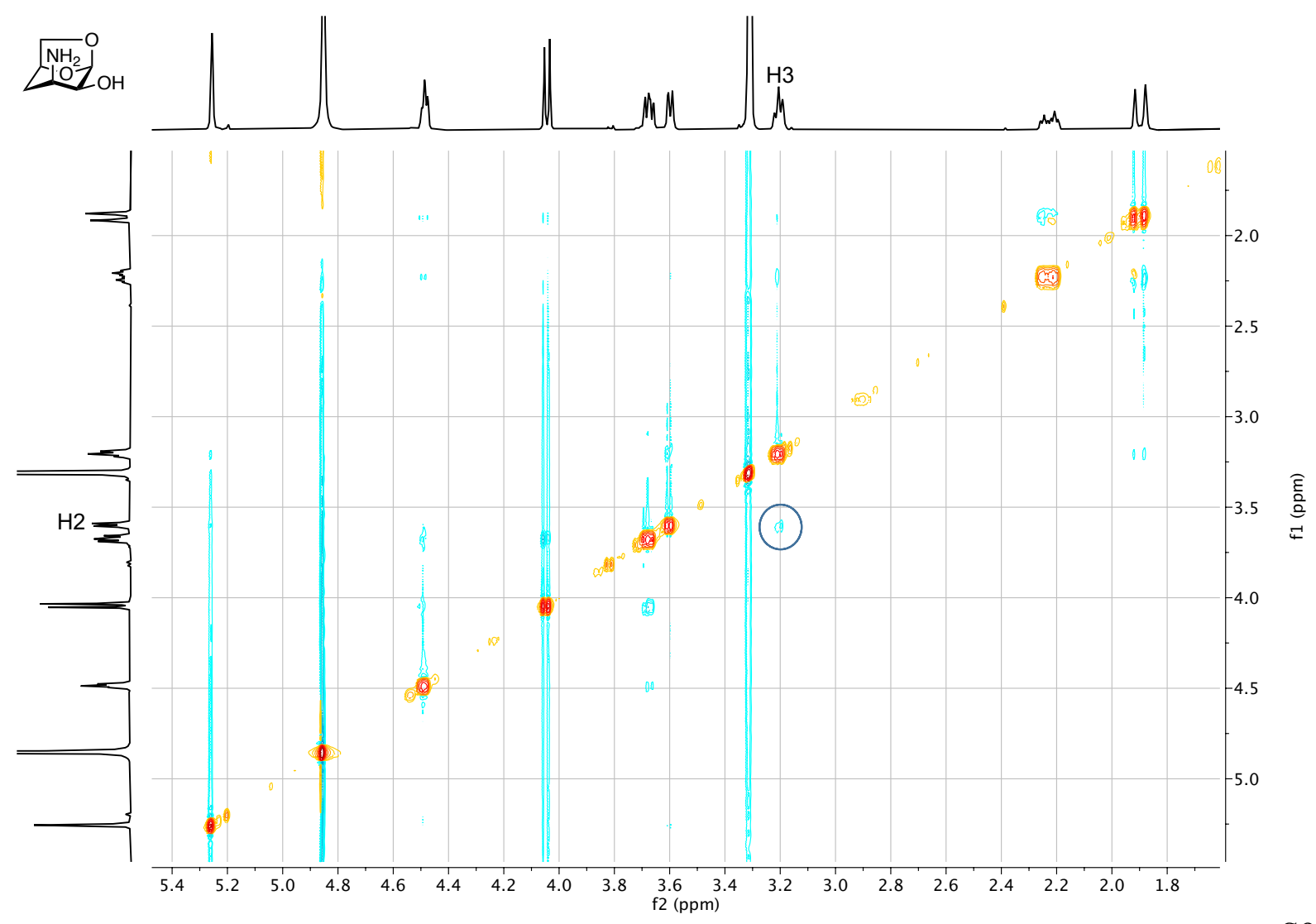

